www.anatomy.org.tr

doi:10.2399/ana.20.002s

\title{
anatomy
}

\section{Abstracts for the 18th Turkish Neuroscience Congress 6-9 November 2020, Ankara, Turkey}

\section{Conferences}

$$
\text { (C-01 - C-10) }
$$

\section{C-01}

Cerebellum and cognition

Hakan Gürvit

Behavioral Neurology and Movement Disorders Unit, Istanbul Faculty of Medicine, Istanbul University, Istanbul, Turkey

\section{C-02}

Brain aging: determinants that could lead to or prevent cognitive decline

Michelle Adams ${ }^{1-3}$

'Department of Psychology, Bilkent University, Ankara, Turkey; 2Interdisciplinary Program in Neuroscience, Aysel Sabuncu Brain Research Center, Bilkent University, Ankara, Turkey; ${ }^{3}$ Interdisciplinary Program in Neuroscience, Aysel Sabuncu Brain Research Center, Bilkent University, Ankara, Turkey

\section{C-03}

What the brain oscillations tell us?

Canan-Başar Eroğlu

Department of Psychology, Izmir University of Economics, Izmir, Turkey

\section{C-04}

Brain dynamics and flexible behaviors

Lucina Uddin

Department of Psychology, Miami University, Miami, FL, USA

\section{C-05}

In-vivo imaging of brain iron and myelin using MRI Jongho Lee ${ }^{1,2}$

'Department of Electrical and Computer Engineering, Seoul National University, Seoul, South Korea; ${ }^{2}$ Department of Brain Cognitive Science, Seoul National University, Seoul, South Korea
C-06

Critical role of cerebral microcirculation in neurodegeneration and neuroprotection

Turgay Dalkara

The Institute of Neurological Sciences and Psychiatry, Hacettepe University, Ankara, Turkey

\section{C-07}

Contextual influences on visual processing

Tom Albright

Salk Institute, La Jolla, CA, USA

\section{C-08}

Perceptual learning and neural plasticity in adult human brain

Fang Fang ${ }^{1,2}$

'Department of Psychology, Peking University, Beijing, China; ${ }^{2}$ The IDG/McGovern Institute for Brain Research, Peking University, Beijing, China

\section{C-09}

Neurovascular Inflammatory Mechanisms of Headache Hayrunnisa Bolay Belen ${ }^{1,2}$

'Department of Neurology, Faculty of Medicine, Gazi University, Ankara, Turkey; ${ }^{2}$ Neuropsychiatry Center, Gazi University, Ankara, Turkey

\section{C-10}

Motor neuron biology and novel insights into the molecular mechanisms of motor neuron degeneration Emel Ulupinar

Department of Interdisciplinary Neuroscience, Health Sciences Institute, Eskişehir Osmangazi University, Eskişehir, Turkey 


\section{Panels}

(PS1 - PS15)

\section{Panel 1 \\ New Techniques in Neuroscience Studies and Strategies for Drug Delivery to the Brain}

\section{PS1-1}

The novel tissue clearing methods in neuroscience for 3D imaging

Esra Özkan, Yasemin Gürsoy Özdemir

Koç University, Istanbul, Turkey

Objective: Traditional histological techniques provide limited information about the 3D structure of the tissue. New microscope technologies enable researchers to take high resolution images in 3D. Rapid and volumetric imaging have been achieved following the invention of light sheet microscopy. To take images with the light sheet microscopy, the transparency of the specimen is required. As a result, tissue clearing became a popular technique which is widely used by scientist in neuroscience. In this presentation, current tissue transparency methods will be explained, and the future directions will be mentioned.

Methods: Today, there are three main methods of tissue transparency which are namely hydrophobic, hydrophilic and hydrogel-based transparency methods. It is possible to make the tissue transparent in a short time with an organic solvent by using the hydrophobic method. However, the loss of fluorescent proteins and significant shrinkage of tissue is observed.

Results: Hydrophilic method provides tissue clearing by involving water soluble agents. One of the disadvantages of this method is the long incubation time required. Lastly, hydrogel-based method involves a specific hydrogel solution with acrylic. Hydrogel forms covalent bonds throughout the tissue and protects the three-dimensional structure. With this method, the tissue is transformed into a solid hydrogel-tissue hybrid. This method allows preservation of the natural protein structure, but the process is time consuming.

Conclusion: The tissue clearing methods are powerful tools for tracking neuronal growth, mapping cerebral circulation network, and tracking specific structures in the embryonic brain. The accessibility of these methods and training of experts to analyse the large amount of data, can be considered as significant problems in scientific research. In the future, the tissue clearing methods may contribute drug development processes and the single cell-level resolution, which is provided by these techniques, may play an important role on early detection of metastatic cancer cells.

Keywords: tissue, clearing, microscopy
PS1-2

3D Co-culture platforms for gene and molecular delivery studies in neuroscience

Emel Sokullu ${ }^{1,2}$

'Department of Biophysics, Faculty of Medicine, Koç University, Istanbul, Turkey; ${ }^{2}$ Translational Medicine Research Center, KUTTAM, Koç University, Istanbul, Turkey

Objective: Tissue engineering is one of the most recent studies to make modeling in order to optimize the tissues that cannot be adequately structurally and functionally. 3D-Coculture platforms are widely used for gene and molecular delivery studies.

Methods: In this study, gelatin methacryloyl (GelMA) synthesis was performed. GelMA polymerization was performed with ultraviolet ray (UV) using photomask with the patented device. Polymerized hydrogel human umbilical vein endothelial (HUVEC) and neuroblastoma (SH-SY5Y) cells were encapsulated. The effects of marine-derived 'Citreohybridonol' molecule doped into GelMA in different ratios on the co-cultured cells were investigated. Fourier transform infrared spectroscopy (FTIR), scanning electron microscopy (SEM), atomic force microscopy (AFM) analyzes were performed to characterize the synthesized GelMA polymer. Cell viability was observed by methylthiazole diphenyltetrazolium (MTT) and hydrogen peroxide (H2O2) for 14 days.

Results: Numerical data obtained from viability analysis were analyzed by GraphPad Prism 8 two-way ANOVA and Tukey test as post hoc. Immunohistochemical staining was performed by using Angiopoietin 1 polyclonal antibody for HUVEC cell line and Nestinantibody for SH-SY5Y cell line in order to observe the molecular effect. The obtained images were found to support cell viability analysis.

Conclusion: In all analyzes, the marine-induced Citreohybridonol molecule did not have a negative effect on the HUVEC cell line. In SH-SY5Y cell line, it was found to have a negative effect on cell viability. Overall results stating that $3 \mathrm{D}$ co-culture platforms are useful for imaging and other molecular techniques to study gene delivery and molecular effects on neuroscience.

Keywords: HUVEC, SH-SY5Y, gelatinmethacrylate, hydrogel, photopolymerization, $3 \mathrm{D}$ coculture

\section{PS1-3}

Nanotechnology- based drug delivery systems for targeted therapy of neurological diseases and brain tumors

Sibel Bozdağ Pehlivan

Department of Pharmaceutical Technology, Faculty of Pharmacy, Hacettepe University, Ankara, Turkey 
Objective: Neurological disorders and brain tumors are major central nervous system (CNS) disorders which significantly treat to human health. Targeting these diseases specifically is challenging, due to the complex nature of the CNS, including the difficulty of bypassing the brain's natural defense systems such as blood-brain barrier (BBB) which allows the entry of only $1 \%$ of drugs, and hence requires special attention so that new treatment strategies can be developed.

Methods: The currently available treatment approaches have led to many side effects and/or are not effective enough. In addition, invasive treatment approaches are no longer favored by patients, as well as by clinicians, hence they are considered to be the last step for treatment. Therefore, new brain-targeted treatment strategies have become a need in the clinical and scientific fields of neurological diseases and neurooncology. Nanotechnology can offer impressive improvement of the treatment of these diseases by using bio-engineered systems interacting with biological systems at a molecular level.

Results: Nanotechnology-based drug delivery systems (NBDDS) including nanoparticles, sizing range 1-1000 $\mathrm{nm}$ have been utilised to cross BBB delivers the drug at cell levels with enhanced bioavailability and reduced toxicity. In overcoming the $\mathrm{BBB}$ with nanoparticulate drug delivery systems, intravenous and intranasal routes which are non-invasive drug delivery routes are commonly preffered.

Conclusion: Different formulation/functionalization strategies such as enhancing the surface properties of nanoparticles by targeting or coating materials or combining different properties of nanostructures in one nanoparticulate system such as hybrid nanoparticles, are implemented the nanoparticulate drug delivery sytems in order to provide more effective therapy.

Keywords: nanotechnology, drug delivery systems, brain

\section{PS1-4}

\section{Microneedle mediated drug delivery for neurological disorders}

\section{Emine Şekerdağ}

Koç University Research Center for Translational Medicine, Koç University, Istanbul, Turkey

Objective: New technologies are emerging to explore hurdles of drug delivery of potential drugs to the brain due to difficulties with passing through the Blood-Brain-Barrier (BBB).

Methods: The BBB can be overcome by using nanoparticulate drug carrier formulations such as liposomes or nanoparticles. However, these formulations normally need to be delivered parenterally (with a needle and syringe) into the body. For neurological disorders, where conventional administration routes are not effective or applicable for the patients, it is very inconvenient and difficult for patients to self-administer those injections which cause stress and pain. Therefore, there is a clear need for novel delivery technologies for neurological drugs. Microneedle technologies are for this purpose very promising, since they allow for minimally-invasive and pain free delivery of nanoparticulate for- mulations and allow self-administration. Microneedles are structures with a length of less than $1 \mathrm{~mm}$ and a diameter in the micrometer range, which are used to deliver drugs across the skin barrier. Microneedle mediated drug delivery systems are primarily based on the subcutaneous/intradermal and transdermal routes. After penetration through the stratum corneum, the drug passes through the viable epidermis and dermis respectively and becomes available for systemic absorption through blood-vessels located in the dermis.

Results: There are several types of microneedle fabrication including solid, hollow, coated, dissolving and hydrogel-forming microneedles. Also, different application approaches are present for these microneedles with each having its advantages and limitations. The main advantages of microneedle mediated drug delivery over oral and injection routes include drug delivery at a controlled rate, non-invasiveness, controlled high blood concentrations, and simple to use.

Conclusion: Combining therapeutic agents for neurological disorders with microneedle mediated drug delivery approach can enhance the bioavailability of the drug and therefore lead to a more uniform drug plasma level that can increase efficacy and reduce adverse events leading to improved patient compliance.

Keywords: neurological disorders, microneedles, drug delivery sistems

\section{Panel 2}

Different Perspectives on Neuron Development and Loss in the Adult Brain

\section{PS2-1}

The role of exosomes in neurogenesis and neurodegeneration

Mehmet Ozansoy

Department of Physiology, School of Medicine, Bahçeşehir University, Istanbul, Turkey

Objective: Adult neurogenesis and neurodegeneration constitute the two important subjects widely investigated in recent scientific literature. In these processes the roles of exosomes has been gaining considerable attention and importance. Exosome with a $20-150 \mathrm{~nm}$ diameter, a well-known component of extracellular vesicles category, is generated by the intraluminal invagination of late-endosomal membrane. They play important roles in intercellular communication through carrying specific cargoes, and they can be found in almost all body fluids, including cerebrospinal fluid (CSF). Exosomes control and regulate the activities of cells by transferring proteins, mRNAs and miRNAs. The aim of this presentation is to discuss the roles of exosomes in neurogenesis and neurodegeneration.

Methods: The development of neuromuscular junction in Drosophila larvae is coordinated by the pre- and post-synaptic proteins carried by exosomes. Exosomes secreted by the oligodendrocytes affect the firing rate, gene expression and signaling pathways in cultured primary neurons. It is also known that exo- 
somes in embryonic CSF promote neural stem cell amplification and in the adul stage exosomes affect the proliferation and differentiation of neural progenitor cells by controlling the molecular components of neurogenic niches. Exosomes derived from induced-neural progenitor cells trigger the proliferation of these progenitors by their miRNA cargoes.

Results: Another important role of exosomes is though their effects in neurodegeneration. In Alzheimer Disease, tau protein and the fragments of amyloid-beta carried by the exosomes contribute the spreading of neuropathology. On the other hand, amyloid-beta fragments carried by the exosomes are taken up by the microglia, microglial degradation of these fragments provides neuroprotection. In Parkinson Disease, exosomes trigger the spreading of pathology by carrying alpha-synuclein.

Conclusion: Exosomes with their diverse roles in neurogenesis and neurodegeneration have become the key players in order to understand the cellular and molecular mechanisms underlying these processes.

Keywords: exosome, neurogenesis, neurodegeneration

\section{PS2-2}

\section{Oligodendrocyte interactome in healthy and diseased nervous system}

$\underline{B i l a l ~ E r s e n ~ K e r m a n ~}^{1}$, Fatmagül İlayda Aydınlı ${ }^{1}$, Burcu Kurt Vatandaşlar $^{1}$, Kübra Yurduseven ${ }^{2}$, Emre Vatandaşlar ${ }^{1}$, Eşref Çelik ${ }^{1}$, Sibel Çimen Yetişs ${ }^{3}$, Abdulkerim Çapar ${ }^{4}$, Zeynep Aladag ${ }^{1}$, Dursun Ali Ekinci ${ }^{4}$, Umut Engin Ayten ${ }^{3}$, Behçet Uğur Töreyin ${ }^{4}$ Işıl Aksan Kurnaz²

'Department of Histology and Embryology, Istanbul Medipol University, Istanbul, Turkey; '2Department of Molecular Biology and Genetics, Gebze Technical University, Kocaeli, Turkey; ${ }^{3}$ Department of Electronics and Communication Engineering, Yıldız Technical University, Istanbul, Turkey; 'Istanbul Technical University, Informatics Institute, Istanbul, Turkey

Objective: Myelin is essential for a healthy nervous system. Myelin formed by oligodendrocytes, accelerates impulse propagation and supports neuronal survival. Demyelination leads to neurodegeneration. In multiple sclerosis (MS) immune attack results in demyelination. Our goal is to dissect interactions among oligodendrocytes, neurons, and immune cells to improve our understanding of myelination and the demyelinating diseases. We aim to discover new targets for remyelination therapies.

Methods: We are building tools for analyzing protein-protein and cell-to-cell interactions. To identify genes involved in myelination and MS, we developed a bioinformatics-based strategy, interactome analysis, which combines proteome and gene expression methodologies. Identified genes are evaluated in peripheral blood of MS patients and in mouse models. To accelerate drug discovery, 23 machine learning-based methodologies were assessed for myelin identification in fluorescent microscopy images.

Results: Interactome analysis identified hundreds of proteinprotein interactions between pairs of oligodendrocytes, neurons, macrophages, microglia, and $\mathrm{T}$ cells. Most significant interactions are further analyzed in vivo and in vitro. Our customizedconvolutional neural network and Boosted Tree methods segmented myelin at over $98 \%$ accuracy. Identified myelin can be quantified and visualized in $3 \mathrm{D}$.

Conclusion: The interactome analysis yielded novel genes that are likely to be linked to MS. Machine learning-based methodologies are very effective in accelerating myelin quantification and thus, drug screens against demyelinating diseases such as MS. Overall, our innovative analysis strategies employing computer assistance produced novel avenues of exploration for myelination and demyelinating diseases.

This study was supported TUBITAK (218S495,316S026), Istanbul Medipol University (BAP2018/06), and Turkish Academy of Sciences (GEBIP).

Keywords: oligodendrocyte, myelin quantification, multiple sclerosis, machine learning, image analysis

\section{PS2-3}

Neural stem cell plasticity in Alzheimer's disease Çağhan Kızıl

German Center for Neurodegenerative Diseases (DZNE), Helmholtz Association, Bonn, Germany

Objective: Alzheimer's disease (AD) is the most prevalent neurodegenerative disease and is incurable. We do not know the exact cause or how to revert the disease manifestation. The neurocentric view of $\mathrm{AD}$, which proposed that the neurons are the centrally affected cells and a treatment could be possible by focusing on these cell types, evolved into a more complex and grift paradigm where many non-neuronal cell types are now thought to be causative to the onset of AD: immune cells, neurovascular unit, and neural stem cells. All these cell types malfunction in $\mathrm{AD}$ and contribute to the complex pathological output. In our lab, we hypothesized that providing new neurons into the diseases brain would be a way to circumvent the effects of $\mathrm{AD}$. One specialized cell type that is capable of doing so is the endogenous neural stem cells, which could act as reservoirs for new neurons; however, lose their ability to do so in disease conditions.

Methods: We are investigating the molecular mechanisms through which human neural stem cells could be coaxed to be plastic - proliferative and neurogenic - again in $\mathrm{AD}$, and we do so by using zebrafish - an organism with extraordinary neuroregenerative ability.

Results: We identified that with what we learn from zebrafish, we can design novel therapeutic ways to fight $\mathrm{AD}$ from a neuro-regenerative perspective.

Conclusion: We will present (1) our adult zebrafish brain model of AD and its regenerative ability, (2) the molecular programs that we identified to enable a regenerative response after Alzheimer's-like loss of neurons, (3) a novel 3D culture method for experimentally modeling human neural stem cell plasticity, 
and (4) our current efforts to translate the findings in zebrafish to humanized systems.

Keywords: zebrafish, neural regeneration, stem cell

\section{Panel 3}

3D Modeling and Simulation with Imaging in Neuroscience

\section{PS3-1}

Three-dimensional (3D) modeling and simulation with imaging in neuroscience: anatomy perspective İlkan Tatar

Department of Anatomy, Faculty of Medicine, Hacettepe University, Ankara, Turkey

Objective: Clinical / surgical Neuroanatomy training is important in learning both macro / micro systems and networks, as well as gaining surgical skills and techniques in neuroscience. This includes basic anatomical structures as well as the complex network of relationships that change in each individual during different pathologies. One of the most important stages of internalizing this whole is 3-dimensional (3D) perception and spatial thinking. The best simulation environment for human anatomy is cadavers. Recently, cadaver supply limitations and the variability in the cost-effectiveness of models have led educators to use more advanced technologies for student-centered practices that facilitate teamwork and improve academic performance.

Methods: Hacettepe University Faculty of Medicine, which prioritizes the vertical integration of basic and clinical sciences between phases in medical education since its establishment, has been the first educational institution to include cross-sectional and clinical anatomy lessons in its curriculum. Since the early 2000s, in the thesis and projects of the Anatomy department, digital photogrammetry, stereoanaglyphic imaging, cadaveric and artistic 3D modeling / printing, virtual, augmented and mixed reality environments were used to increase both undergraduate and graduate student's 3D perception and thinking together skills about anatomical structures.

Results: In recent years the Surgical NeuroAnatomy group was established, aiming to improve the surgical skills of graduate students by supporting cadaver dissections with digital or physical $3 \mathrm{D}$ models. For this, by using very different imaging techniques and sequences, tissues and organs with different mechanical and biological properties were modeled as close to their anatomical reality and these models were used both in surgical planning and training.

Conclusion: It has been observed in our researches and completed doctoral dissertations that the use of physical models, in accordance with the literature on this subject, is more effective and reinforcing in basic undergraduate education and the digital ones in postgraduate education and surgical planning.

Keywords: 3D physical/digital modeling, Surgical/Clinical NeuroAnatomy, simulation, surgical planing
PS3-2

Three dimensional (3D) modelling and simulation with imaging in neurosciences: neurosurgical perspective Melike Mut Aş̧un, Şahin Hanalioğlu, İlkay Işıkay

Department of Neurosurgery, Faculty of Medicine, Hacettepe University, Ankara, Turkey

Objective: "Seeing is undertanding in science". Learning and transferring the knowledge of neuroanatomy is fundemental in neurosurgery. Besides basic anatomy; it is essential to understand how the anatomy may show variations in each patient and how the relation of normal anatomical structures can change in the presence of pathology. Transformation of two-dimensional neuroradiological images into $3 \mathrm{D}$ spatial models are critical in understanding normal physiology and pathophysiological mechanisms.

Methods: With the initiative of Hacettepe University Neurosurgery, Neuroradiology and Anatomy, preoperative neuroradiological data of our patients were modeled in $3 \mathrm{D}$. Computed tomography and magnetic resonance imaging sequences, in which bone, brain, vascular and nerve tissues are best viewed, were selected; 3D planning and modeling was transferred to the Mimics Innovation Suite software, and segmentation and modeling processes were performed with this software. The obtained virtual models were transferred to the digital platform and printed models with texture characteristics closest to reality were produced in $3 \mathrm{D}$ printer. These models have been used in both surgical planning and specialty training.

Results: 3D modeling has been used in pituitary, skull base and brain tumors and aneurysm cases. Before surgery, different tissues were segmented in layers, photgraphic images were taken in standard anatomical and surgical positions, and surgical approach methods were studied. The planning on the preoperative $3 \mathrm{D}$ model and intraoperative images were found to be highly compatible.

Conclusion: Creation of the patient specific 3D models based on the detailed neuroradiological images has been transferred to the surgical field. Widespread use of 3D modelling will reduce surgical complications, shorten the duration of the operation, and perform more effective surgery by preserving the function. By combining 3D modeling with VR/AR techniques, it will be possible to develop customized simulators that can be used for surgical planning and training in the near future.

Keywords: 3D modeling, segmentation, radiological imaging, neurosurgery

\section{PS3-3}

Three dimensional (3D) modelling and simulation with imaging in neuroscience: radiological perspective

Kader Karlı Oğuz, Gökçen Çoban Çiftçi

Department of Radiology, Faculty of Medicine, Hacettepe University, Ankara, Turkey 
Objective: To emphasize critically important issues during use of imaging techniques in 3D modelling.

Methods: Computed Tomography (CT) imaging is ideal for bone and different algorithms should be used to separate high density nonosseous structures adjacent to the bones.Vascular structures are segmented from arterial or venous phase CT and/or MR Angiograms. Calcified plaques on vessel wall and adjacent bone or hematoma should be separated using both angiograms. Ideal imaging is Magnetic Resonance Imaging (MRI) for brain parenchyma including structural T1, T2weighted and FLAIR, Gd-T1W, diffusion weighted imaging. For skull-base masses like chordoma, bony structures on CT, mass on MRI and vascular structures on CTA/MRA are coregistered. Neural structures traversing cerebrospinal fluid can be nicely delineated using steady-state 3D high resolution technique.

Results: A 3D modelling example of a microadenoma in very complicated structural complex-sellar region will be shown to the audience.

Conclusion: Using appropriate imaging techniques, 3D models of intracranial lesions in very complicated regions can be created for pre-operative personal surgical planning.

Keywords: aneursym, imaging, neurosurgery, three dimensional modelling, tumour

\section{PS3-4}

Three-dimensional (3D) modeling and simulation with imaging in neuroscience: instructional technologies perspective

Cengiz Savaş Aşkun

Middle East Technical University, Ankara, Turkey

Objective: Neuroanatomy training plays an essential role in the training of neurosurgery residents. The traditional approach to residency training includes hands-on lessons on cadavers before taking part in real surgical experience. However, residents find it difficult to spare time for the neuroanatomy laboratory due to their busy schedule in the clinic. The use of Learning Management Systems (LMS) allows institutions to transfer lessons to digital space allowing anytime, anywhere interaction among tutors and learners. This has become even more important during the COVID-19 outbreak. Our aim is to design an online platform through which neuroanatomical and patient / disease-based 3D models and simulation are presented.

Methods: Moodle 3.9 and various 3D modelling tools were used to create an online learning platform. 3D patient models based on neuroradiological data obtained from Hacettepe University Faculty of Medicine were uploaded into the system and tested. $3 \mathrm{D}$ models were compared with cadavers and real-time intraoperative microscopic, neuronavigation data. Using Moodle 3.9, an infrastructure has been established to be able to present $3 \mathrm{D}$ models in addition to theoretical course materials to a group of neurosurgery residents. In order to enable more participation online quizzes and interactive materials were used.
Results: Promising results were obtained when 3D models were evaluated by instructors and residents in terms of their suitability to reality, user-friendliness, compatibility of computer and mobile interfaces and interaction.

Conclusion: The system will serve as a platform where assistants will examine patient data, plan the pre-operative approach, and finally discuss their experiences. Future research plans include using 3D models of real patients and post-operative analysis of their surgical experience so that residents can use the system before participating in a surgical operation.

Keywords: 3D modeling, instructional technology, learning management systems (LMS), online education

\section{Panel 4}

Neuroinflammation

\section{PS4-1}

\section{Neuroinflammation}

Turgay Dalkara, Evren Erdener, Emine Eren Koçak, Asli Tuncer

Hacettepe University, Ankara, Turkey

Neuroinflammation, although popular, is a rather vaguely defined term. It exhibits substantially different features than systemic inflammation and also spans a wide spectrum of phenotypes. On one hand, mild perturbations in brain parenchyma leads to activation of a neuroinflammatory signaling cascade that encompasses canonical features of inflammation such as inflammasome formation, caspase- 1 activation, high-mobility group box 1 (HMGB1) and interleukin-1, release from cells culminating in translocation of NF-kappa B to nucleus and induction of pro-inflammatory enzymes. This self-limited signalling pathway functions to alert the organism by causing pain and triggering the repair mechanisms. An example of this is the cortical spreading depolarization (biological correlate of migraine aura)-induced inflammatory signalling and headache seen in migraine. Transient but intense metabolic challenge and cellular swelling seen during cortical spreading depolarization trigger the inflammatory response. On the other hand, in depression, there are no known signs of cellular perturbation other than altered synaptic transmission homeostasis but many indications of a brain-derived inflammatory signalling. On the other hand of the spectrum, multiple sclerosis damages the brain and spinal cord with an inflammatory reaction that shares several common features with an auto-immune disease but yet with its unique character. In this symposium, this wide spectrum of inflammation in the brain will be discussed in the context of the cortical spreading depolarization (Dr. Evren Erdener), depression (Dr. Emine Eren Koçak) and multiple sclerosis (Dr. Asli Tuncer) after introduction of the neuroinflammatory cascade and its self-limiting features (Dr. Turgay Dalkara).

Keywords: depolarization, multiple sclerosis, depression 
Panel 5

The Neural Basis of Number Perception and Dyscalculia: Structural, Functional, Developmental Assessment and Disease Signature

\section{PS5-1}

Structural brain connectivity differences between children with developmental dyscalculia and typically developing peers

Nazife Ayyıldız ${ }^{1}$, Frauke Beyer ${ }^{2}$, Sertaç Üstün ${ }^{3}$, Emre H. Kale ${ }^{4}$, Öykü Mançe Çalışır ${ }^{5}$, Pınar Uran ${ }^{6}$, Özgür Öner ${ }^{7}$, Sinan Olkun ${ }^{8}$, Alfred Anwander ${ }^{9}$, Veronica A. Witte ${ }^{2}$, Arno Villringer ${ }^{2}$, Metehan Çiçek ${ }^{3}$

'Department of Interdisciplinary Neuroscience, Health Sciences Institute, Ankara University, Ankara, Turkey; 2Department of Neurology, Max Planck Institute for Cognitive and Brain Sciences, Leipzig, Germany; ${ }^{3}$ Department of Physiology, School of Medicine, Ankara University, Ankara, Turkey; ${ }^{4}$ Brain Research Center, Ankara University, Ankara, Turkey; ${ }^{5}$ Program of Counseling and Guidance, Department of Educational Sciences, Faculty of Educational Sciences, Ankara University; Ankara, Turkey; ${ }^{6}$ Department of Child and Adolescent Psychiatry, School of Medicine, Ankara University, Ankara, Turkey; 'Department of Child and Adolescence Psychiatry, School of Medicine, Bahçeşehir University, Istanbul, Turkey; ${ }^{8}$ Department of Mathematics Education, Final International University, Kyrenia, Cyprus; ${ }^{9}$ Department of Neuropsychology, Max Planck Institute for Human Cognitive and Brain Sciences, Leipzig, Germany

Objective: Developmental dyscalculia (DD) is a specific learning disability that negatively affects individual's arithmetic learning. Difficulties often continue from childhood through adulthood. Underlying neurobiological mechanisms of DD, however, have not been fully understood. The purpose of this study was to investigate possible structural brain connectivity differences between children with DD and typically developing (TD) peers.

Methods: After screening 2058 third grade students in elementary schools with mathematical tests, we assigned children to DD and TD groups according to the screening results. After two years later, we invited children for detailed neuropsychological assessments. We obtained pure DD and TD children sample excluding comorbidities. Volunteer children were familiarized with a mock MRI-scanner. We acquired Diffusion Tensor Imaging (DTI) data with a Siemens Magnetom-Trio-Tim 3Tscanner with 60-different phase-encoding directions.

Results: After preprocessing, we evaluated 10 children with pure DD $(11.3 \pm 0.7$ years $)$ and 16 TD peers $(11.2 \pm 0.6$ years $)$. We first assessed white matter microstructure with tract-based spatial statistics. Then we used probabilistic tractography to evaluate tract lengths and probabilistic connectivity maps in specific ROIs (Regions-of-Interest). At whole-brain level, we found no significant microstructural differences in white matter between DD children and TD peers. Also, seed-based connectivity probabilities showed no differences between groups. However, we found significant differences in ROI-tracts which had previously been related to math ability in children. The major findings of our study were reduced white matter integrity and shorter fiber lengths of the left superior longitudinal/arcuate fasciculus and left anterior thalamic radiation in the DD group.

Conclusion: These results from regional analyses indicate that learning, memory and language-related pathways in the left hemisphere might underlie DD. Further studies are needed to implement with larger data sets, using longitudinal and/or interventional designs to confirm these findings. (This study was supported by TÜBİTAK under the project code 214S069).

Keywords: Mathematical learning disability, diffusion tensor imaging, superior longitudinal fasciculus, anterior thalamic radiation

\section{PS5-2}

Effective connectivity provides a disease signature for dyscalculia: an fMRI study

Salih Geduk $^{1}$, İlkay Ulusoy ${ }^{1}$, Sertaç Üstün ${ }^{2}$, Nazife Ayyıldız Gözde Vatansever ${ }^{4}$, Pınar Uran ${ }^{5}$, Özgür Öner ${ }^{6}$, Sinan Olkun, Metehan Çiçek ${ }^{2}$

'Department of Electrical and Electronics Engineering, Middle East Technical University, Ankara, Turkey; 'Department of Physiology, Ankara University School of Medicine, Ankara, Turkey; ${ }^{3}$ Department of Interdisciplinary Neuroscience, Institute of Health Sciences, Ankara University, Ankara, Turkey; "Brain Research Center, Ankara University, Ankara, Turkey; ${ }^{5}$ Department of Child Psychiatry, Ankara University School of Medicine, Ankara, Turkey; ${ }^{6}$ Department of Child Psychiatry, Bahçeşehir University School of Medicine, Istanbul, Turkey; 'Department of Mathematics Education, Final International University, Kyrenia via Mersin 10 Turkey, TRNC

Objective: Developmental dyscalculia (DD) is a specific learning disability characterized by a deficiency in math learning. It is suggested that dyscalculia might be caused by a connectivity deficit in the brain. The main purpose of our study was to provide a method to assist the diagnosis of dyscalculia using effective connectivity modeled by discrete Dynamic Bayesian Network (dDBN).

Methods: dDBN was modeled using the functional magnetic resonance imaging (fMRI) data obtained from twelve children with dyscalculia (8 female, mean age: 11.25$)$ and fifteen typically developing children ( 9 female, mean age: 11.26 ) volunteers while they were performing a numerosity task. The approach consisted of three steps. Firstly, imaging data were analyzed by repeated-measures ANOVA with group (dyscalculic/control), number modality (symbolic/non-symbolic tasks), and difficulty (0.7/0.5 ratios) as factors, using SPM12 software. Significantly activated voxel clusters related to the main effect of group were selected as the region of interests (ROI). There were six ROIs: the left and right intraparietal sulci, right anterior cingulate cortex, left medial prefrontal cortex, left hippocampus, and occipital cortex. Secondly, dDBN was used to model the brain connectivity using the fMRI time series data of these ROIs. The effective connectivity is modeled for each task by using two methods, which were virtual-typical-subject and individual-structure methods. Finally, the Bayesian score was used to classify dyscalculic participants from control participants for each method separately.

Results: A prefrontal-parietal and hippocampal network in the left hemisphere shows effective connectivity in dyscalculia, which 
might be the result of their brain's compensation mechanisms. In addition, when the connectivity maps of the symbolic task were used, the classification performance reached $70 \%$ accuracy.

Conclusion: Hyper-connectivity was observed in children with DD. The significant connectivity differences are mainly reported in the left hemisphere for the symbolic task.

This study was supported within the scope of TUBITAK project number 214S069.

Keywords: dyscalculia, fMRI, effective connectivity, disease signature, discrete Dynamic Bayesian Network

\section{PS5-3}

Children with dyscalculia show hippocampal hyperactivity during symbolic number perception

Sertaç Üstün $^{1}$, Nazife Ayyıldız ${ }^{2}$, Emre H. Kale ${ }^{3}$, Öykü Mançe Çalışır ${ }^{4}$, Pınar Uran ${ }^{5}$, Özgür Öner ${ }^{6}$, Sinan Olkun, Metehan Çiçek ${ }^{1}$

'Department of Physiology, School of Medicine, Ankara University, Ankara, Turkey; ' Department of Interdisciplinary Neuroscience, Ankara University, Ankara, Turkey; ${ }^{3}$ Brain Research Center, Ankara University, Ankara, Turkey; "Program of Counseling and Guidance, Department of Educational Sciences, Faculty of Educational Sciences, Ankara University, Ankara, Turkey; ${ }^{5}$ Department of Child and Adolescent Psychiatry, School of Medicine, Ankara University, Ankara, Turkey; ${ }^{6}$ Department of Child and Adolescence Psychiatry, School of Medicine, Bahçeşehir University, Istanbul, Turkey; 'Department of Mathematics Education, Final International University, Kyrenia, Cyprus

Objective: Dyscalculia is a learning disability affecting the acquisition of arithmetical skills in children with normal intelligence and age-appropriate education. Two main hypotheses attempt to explain the cause of dyscalculia. The first hypothesis suggests that a problem with the core mechanisms of perceiving (non-symbolic) quantities is the cause of dyscalculia, while the alternative hypothesis suggests that dyscalculics have problems only with the processing of numerical symbols. In this study, number processing in children with dyscalculia and typically developing children was examined via functional magnetic resonance imaging (fMRI).

Methods: In the first stage of the study, 2058 third-grade students are scanned with arithmetic tests. After the determined children evaluated by a child psychiatrist, the dyscalculia $(n=12$, mean age: 11.25$)$ and control $(\mathrm{n}=15$, mean age: 11.26$)$ groups were precisely identified. Participants were asked to perform a numerosity comparison paradigm while undergoing an fMRI scan. The paradigm consists of two types of number conditions (dot and symbolic comparison) and two difficulty levels ( 0.5 and 0.7 ratios).

Results: The results showed that bilateral intraparietal sulcus and dorsolateral prefrontal cortex were activated for number perception. The task difficulty activates bilateral insular and anterior cingulate cortex. The dyscalculia group showed more activation in the left orbitofrontal cortex, left medial prefrontal cortex and right anterior cingulate cortex than the control group. The dyscalculia group showed left hippocampus activation specifically for the symbolic comparison condition.

Conclusion: The frontoparietal network activation in number tasks along with the insular and anterior cingulate cortex activation that related to task difficulty are in line with the literature. Increased left hippocampal and left-lateralized frontal network activation in dyscalculia group suggest that executive control and memory-based compensation mechanisms are required for symbolic processing for dyscalculics. Overall, our findings indicate that dyscalculia is related to difficulty in symbolic processing.

*This study was supported by TÜBİTAK under the project code 214S069.

Keywords: Dyscalculia, number sense, fMRI, frontoparietal network, hippocampus

\section{PS5-4}

Number perception networks in the brain and their developmental alterations

Gözde Vatansever ${ }^{1}$, Sertaç Üstün ${ }^{2}$, Nazife Ayyıldız ${ }^{1}$, Metehan Çiçek ${ }^{2}$

'Department of Interdisciplinary Neuroscience, Ankara University, Ankara, Turkey; ${ }^{2}$ Department of Physiology, School of Medicine, Ankara University, Ankara, Turkey

Objective: The ability to discriminate quantities approximately is known as number perception. Research shows that number perception is a fundamental ability that humans have since infancy. Neuroimaging studies revealed that number perception is mainly located in frontal and parietal areas, especially in the intraparietal sulcus. The developmental course of this number network is still controversial. A group of developmental studies finds an increase of activation in the parietal areas and a decrease of activation in the frontal cortex with age. Based on these findings it was suggested that in childhood, frontal cortices are predominant for numerical processes whereas, with age, parietal areas take over. However, this "fronto-parietal shift theory" is controversial due to a lack of consistency in the field. In our study, we aimed to investigate the developmental differences in the fronto-parietal number network.

Methods: Event-related fMRI data obtained from adult and children groups during numerical paradigm. Sixteen healthy young adults (age: $21.69 \pm 0.79)$ and 15 healthy children (age: $11.87 \pm 0.52$ ) performed a numerosity comparison paradigm which consists of two numerical conditions (dot-symbol) with two difficulty levels (easy-hard).

Results: Adults activated parietal, frontal, cingulate, and peristriate cortices while children activated intraparietal sulcus and peristriate cortex. Difficulty related insula activation was observed in both groups. Group differences were observed in the posterior insula, fusiform gyrus, and peristriate cortex whose coordinates correspond to the number form area(NFA) which is a specific part of the fusiform gyrus that has been found to respond to numbers only.

Conclusion: Overall, our findings suggest developmental changes in the brain's number processing revolving around the 
NFA. In contrast to the prominent fronto-parietal shift theory, no age-related differences were observed in the frontoparietal regions. The role of frontal areas in number processing seems to be critical also in adulthood. This study is partially funded by TÜBİTAK with project number 214S069.

Keywords: developmental differences, fMRI, number form area, number perception

\section{Panel 6}

Oscillatory Dynamics of Cognitive Processing, Healthy Aging, and Cognitive Impairments

\section{PS6-1}

Theta oscillation, the theta system of the brain and information processing correlates

Sirel Karakaş

Department of Psychology, Doğuş University, Istanbul, Turkey

Objective: Theta is an extensively studied oscillation of the nervous system but there is only a paucity of previous on the subject. A review of specifically the cognitive-affective correlates of the theta oscillation is currently unavailable. The present review aims to fill this gap.

Methods: Theta-based hippocampal binding brings together the environmentally triggered multimodal elements of episodes or scenes; this makes multimodal sensory/perceptual and motor processing, facilitatory and inhibitory attention, navigation and episodic memory possible. Hippocampal binding, supramodal associative processing, and associations between theta and other oscillatory components give way to the selectively distributed theta system of the brain. The association between different sources of information and between oscillations of different frequency bands, the connectivity in the theta network and coherences between selected brain areas contribute to the synchrony and hypersynchrony of the human brain. Therefore, the ultimate outcome in information processing is a function of the connectivity between relevant brain areas and the ensuing selectively hypersynchronous brain through which theta transfer occurs.

Results: Hippocampus is responsible for memory. However, hippocampus performs this function in close collaboration with the prefrontal cortex, and ultimately the whole brain. Theta, the major operating rhythm of the selectively distributed network responds to different phases of memory (acquisition, consolidation, retrieval) via different parametric responses (e.g., synchronization/desynchronization, coherence, coupling, time-locking, power) during wakefulness and different stages of sleep (deep sleep, rapid eye movements sleep).

Conclusion: Mechanisms of interstructural binding (hippocampus), mechanisms of interstructural association formation and coherence (connectivity of the cortico-hippocampal network) result in a densely connected pool of information. This information travels over an interconnected, and highly synchronized system (cortico-hippocampal system) in the form of theta waves; theta is the major operating rhythm of the system. Theta oscillation represents many of the cognitive-affective processes that cognitive psychology and cognitive neuroscience encompass.

Keywords: theta oscillation, hippocampus, cortico-hippocampal interplay, connectivity, cognitive-affective correlates, memory

\section{PS6-2}

\section{Brain oscillations during healthy aging}

Canan Başar Eroğlu ${ }^{1}$, Mert Küçük ${ }^{1}$, Christina Schmiedt Fehr ${ }^{2}$

'Department of Psychology, Izmir University of Economics, Izmir Turkey; ${ }^{2}$ University of Bremen, Bremen, Germany

Objective: Previous studies on EEG event-related brain oscillations suggest functional changes in delta and theta frequency bands with age during cognitive tasks. Event-related oscillations, (EROs) consider the spectral frequencies underlying and determining the ERP waveform, as the phase and magnitude of different frequency oscillations in single trials determine the ERP components. In older adults a decrease in slow frequency EEG power has been consistently reported. Thus, when estimating age-related changes in higher cognitive processing with electrophysiological measures investigate, it appears reasonable to consider both processing fluctuations in single trials (potentially producing a latency jitter) and age-related changes in EEG-oscillations (also determining the averaged ERP waveform). The aim was to analyze both processes during the healthy aging.

Methods: Present study investigates of the assessed Go- and NoGo-ERP measures with estimations of single trial EEG spectral power (magnitude) and EEG spectral ITC (phase-locking) in delta and theta frequency bands. 16 gender and education matched young and 16 older adult participants for data analysis. All individuals were healthy according to self-report and had normal or corrected-to-normal vision.

Results: Young individuals were significantly faster than older individuals $\left(384 \pm 77\right.$ versus $\left.511 \pm 74 \mathrm{~ms}, \mathrm{df}=30, \mathrm{t}=\_4.75, \mathrm{p}<.001\right)$. Young people showed significantly larger single trial delta magnitude than older adults at all locations and for both Go/NoGo conditions, while no group differences in delta ITC were obtained. These results will be discussed in detail.

Conclusion: These results indicate that the observed agerelated changes in Go and NoGo P3-waves are not related to an increased latency jitter of the delta responses, which is in congruence with previous studies. We conclude that withinperson variability may increase with age, but the degree depends on performance level and the modality investigated and delta and theta oscillations indicate that the elderly are not always old.

Keywords: EEG, delta and theta oscillations, aging 


\section{PS6-3}

Reflections of impaired facial expression perception on EEG-event-related brain oscillations in patients with Parkinson's disease

Bahar Güntekin $^{1}$, Ebru Yıldırım² ${ }^{2}$ İlayda Kıyı ${ }^{3}$, Tuba Aktürk ${ }^{4}$, Hakan Uzunlar ${ }^{5}$, Pervin Çalışoğlu ${ }^{5}$, Görsev Yener ${ }^{6}$

'Department of Biophysics, School of Medicine, Istanbul Medipol University, Istanbul, Turkey; 2Program of Electroneurophysiology, Vocational School, Istanbul Medipol University, Istanbul, Turkey; ${ }^{3}$ Department of Neurosciences, Institute of Health Sciences, Dokuz Eylül University, Izmir, Turkey; "Istanbul Medipol University, Research Institute for Health Sciences and Technologies (SABITA), Regenerative and Restorative Medicine Research Center (REMER), Istanbul, Turkey; ${ }^{5}$ Department of Neuroscience, Graduate School of Health Sciences, Istanbul Medipol University, Istanbul, Turkey; ${ }^{6} / z m i r$ Biomedicine and Genome Center, Dokuz Eylül University Health Campus, Izmir, Turkey

Objective: Facial expression perception is an essential part of human cognitive processes and social interaction. There is a crucial link between emotional processes and human memory; this link could be damaged by impairment of cognitive processes and memory functions. This study aimed to investigate the effect of impaired facial expression perception on EEG-event-related oscillations in Parkinson's patients (PD) with cognitive impairment.

Methods: Theta $(4-7 \mathrm{~Hz})$ and alpha $(8-13 \mathrm{~Hz})$ responses, which are known to increase in the perception of emotional facial expressions, were examined in PD patients and healthy controls. 20 healthy elderly controls, 14 Parkinson's patients with mild cognitive impairment (PD-MCI), and 14 Parkinson's patients with dementia (PD-Dementia) were included in the study. 32 channel of EEG was recorded in all subjects during the facial expression paradigm (angry, happy, neutral). Theta and alpha phase-locking and power spectrum analyses were performed on the time-frequency domain.

Results: The results showed that cognitive impairment in Parkinson's disease negatively affected the perception of facial expressions, and this effect is represented by theta and alpha responses. Healthy controls had significantly lower theta phaselocking and theta power than PD-MCI patients $(\mathrm{p}=0.019$; $\mathrm{p}=0.03)$ and $\mathrm{PH}-$ Dementia patients $(\mathrm{p}=0.005 ; \mathrm{p}=0.003)$. Alpha phase locking of PD-MCI patients $(\mathrm{p}=0.012)$ and PD-Dementia $(\mathrm{p}=0.03)$ patients decreased compared to healthy controls, but the alpha power spectrum values were not affected.

Conclusion: The present study showed that facial expression perception was affected by increased cognitive impairment in patients with Parkinson's disease. This impairment was represented by decreased theta and alpha responses. Impaired facial expression perception, which is one of the crucial processes of social life, can be expected to negatively affect Parkinson's patients' social lives.

This work (grant number 218S314) was supported by TUBITAK.

Keywords: brain oscillations, facial expression, Parkinson's disease, phase-locking, power spectra
PS6-4

Phase locking of event-related oscillations with stimulus may distinguish different stages of cognitive impairment in the Alzheimer's continuum

Emine Elif Tülay ${ }^{1}$, Bahar Güntekin², Görsev Yener ${ }^{3}$, Ali Bayram ${ }^{4}$, Canan Başar Eroğlu ${ }^{5}$, Tamer Demiralp

'Department of Computer Engineering, Üsküdar University, Istanbul, Turkey; ' Department of Biophysics, Istanbul Medipol University, Istanbul, Turkey; ${ }^{3}$ Department of Neurology, Dokuz Eylül University, Izmir, Turkey; ${ }^{4}$ Department of Neuroscience, Istanbul University, Istanbul, Turkey; ${ }^{5}$ Department of Psychology, Izmir University of Economics, Izmir, Turkey; ${ }^{6}$ Department of Physiology, Istanbul University, Istanbul, Turkey

Objective: Current study aimed searching for potential event related oscillation (ERO) biomarkers to distinguish different stages of cognitive decline along the Alzheimer's Diseases (AD) continuum. Another purpose of the present study is to use a rigorous approach for the statistical evaluation of the whole brain EEG data, in order to define the most robust and reproducible ERO features that can easily be tested on other data sets.

Methods: Target responses of a visual oddball experiment recorded from $37 \mathrm{AD}, 46 \mathrm{MCI}$ patients and 48 age, gender, and education matched normal elderly controls were analyzed for both evoked and total ERO powers in the wide frequency range that covers the delta, theta, alpha, beta and gamma bands, by applying continuous complex wavelet transform (WT) on averaged and single trial data, respectively. While preprocessing of the data were performed using BrainVision Analyzer 2.1 (www.brainproducts. com), time-frequency analysis and the cluster-based nonparametric permutation tests for statistical analysis were implemented in the FieldTrip toolbox (www.fieldtriptoolbox.org).

Results: The findings in the comparative analysis of the evoked and total powers along the $\mathrm{AD}$ continuum is that the evoked delta response displays a progressive decline along the course of the disease, reaching significant level in the $\mathrm{AD}$ phase, in contrast to the total delta and theta responses that already were significantly reduced in the MCI phase. There was no significant difference among groups for alpha, beta and gamma frequency bands.

Conclusion: These results suggest that total delta and theta EROs to oddball targets may be useful for early detection of the disease in MCI stage, while the evoked delta response allows following the progress from MCI to AD.

Keywords: EEG, ERO, wavelet analysis, Alzheimer's disease, mild cognitive impairment

\section{Panel 7}

Interdisciplinary Studies in Cognitive Neuroscience and Human-Robot Interaction

\section{PS7-1}

Why do we need an interdisciplinary collaboration between cognitive neuroscience and social robotics?

Burcu Ayşen Ürgen

Bilkent University, Ankara, Turkey 
Objective: With the advances in technology, we are no longer surrounded only by biological organisms such as our conspecifics or other animals. Social robots have been part of our lives for a while, and help us in various domains ranging from education to healthcare to elderly care. For instance, we see robots that tutor students in learning a second language, that participate in rehabilitation of some disorders such as autism or stroke, and that guide us in restaurants, banks, or shopping malls.

Methods: The involvement of robots in our lives has brought up two questions: (1) How do we perceive and interact with artificial agents that we have not evolved with over many generations? (2) How should we design robots so that humans can accept them and interact with them efficiently?

Results: The first question concerns cognitive neuroscience which aims to understand the nature of the human brain and cognition. In this context, artificial agents such as robots are useful experimental tools to understand how humans respond to novel entities that show some similarities with humans (e.g. human-like appearance) but also that diverge from humans in various aspects (e.g. mechanical motion). The second question concerns mainly social robotics and human-robot interaction. Here, the goal is to identify design principles that can guide engineers in robot development. We propose that those effective design principles can be determined by incorporating our knowledge from well-established findings of cognitive neuroscience.

Conclusion: In sum, we believe that an interdisciplinary collaboration between cognitive neuroscience and social robotics is a fruitful one which can address questions about the human brain and mind as well as robot design.

Keywords: cognitive neuroscience, social robotics, visual perception

\section{PS7-2}

Investigation of brain-to-brain synchronization patterns via fNIRS hyperscanning

Murat Perit Çakır

Department of Cognitive Science, METU Informatics Institute, Ankara, Turkey

Increasing availability and robustness of optical and electrophysiological imaging modalities have opened up the possibility of monitoring the brain activities of two or more participants synchronously while they are engaged in various levels of social interaction. In this presentation we will summarize the initial findings of our ongoing hyperscanning studies in a dual fNIRS setup focusing on various social coordination and competition tasks including joint sentence reading, problem solving, game-theoretic decision making tasks. Together with examples from similar fNIRS hyperscanning studies with varying coverage of the cortical surface, we will highlight some of the common synchronization patterns that emerge in the presence and absence of coordination among the interactants. The presentation will conclude with a discussion of the implications of these initial findings in the context of joint brain-computer interfaces and human-machine interaction.

Keywords: fNIRS, hyperscanning, connectivity

\section{PS7-3}

Dividing it into pieces and Bringing them together: interdisciplinary perspectives in cognitive sciences

Cengiz Acartürk

Department of Cognitive Science, METU Informatics Institute, Ankara, Turkey

During the history of research on the interaction between humans and robots the researchers have frequently faced unexpected findings. For example, the feeling of fidelity, that improves as the visual components of humanoid robots are improved, also led to the feeling of uncanny; the interaction between autonomous robots in manufacturing lines and humans resulted in unfortunate accidents; the research studies, that assumed a replacement of humans by robots that have artificial intelligence, have been evolved into the studies of how to design appropriate collaboration between robots and humans. Human Robot Interaction, as a domain of scientific research, is a domain that has been subject to research in specific subdomains and interdisciplinary approaches, due to the complexity of the human cognitive system that reaches at the interaction, the variety in the affordances of the physical or virtual robots, and the richness of the interaction scenarios under investigation. In this presentation, example studies will be presented in the domain of Human Robot Interaction and the contribution of different disciplines to the field will be demonstrated.

Keywords: robot, human-robot interaction, cognitive neuroscience

\section{PS7-4}

The effect of kinemorphs on F-formation shapes: an investigation on human robot interaction in virtual reality

Gizem Özen

Department of Cognitive Science, Informatics Institute, Middle East Technical University, Ankara, Turkey

Objective: The present study aims to investigate how facial kinemorphs influence F-formation shapes occurred in a virtual environment social interaction setting.

Methods: The problem statement relies on two aspects of this investigation, more precisely, two comparative analysis. The first comparison is between the effect of the gaze and the lip movement on F-formation shapes. The importance of this aspect in HRI studies depends on the reason being that it is not clear whether the shape of a social group's spatial arrangement is affected by the kinemorphs, while it is known that kinemorphs have an influence on the proximity between humans and robots. Through the comparative analysis between the gaze and the lip movement, the effect of the kinemorphs on F-formation shapes can be clearly observed. The second comparison is between the interactions of human participants with robots in virtual reality and the interaction between humans in physical settings. It is known that human behavior has different patterns when it is towards a human 
and when it is towards a robot. Therefore, F-formation, as a behavior of the decision on location and rotation, might also have a difference in forming interactions with humans and robots. This difference is revealed by investigating the role of $\mathrm{F}$-formation shapes on joining dyadic and triadic interaction scenarios.

Results: The results evince systematic findings that address likely postures in dyadic and triadic interaction settings for the robot agents in a virtual environment.

Conclusion: Both kinemorphs (gaze and lip movement) seem to determine the focus of the participants in a social interaction with robots; however, the effect of gaze is higher than lip movement. $\mathrm{H}$ and $\mathrm{V}$ formations are the most frequently formed shapes in virtual triadic and dyadic interactions. I formation does not even have a single instance neither in triadic nor in dyadic interactions.

Keywords: F-formation, kinesics, virtual environment, human robot interaction

\section{Panel 8}

\section{Computational Neuroscience: From Cortical Information Representations to Brain-Machine Interfaces}

\section{PS8-1}

Deep learning: a machine learning algorithm in computational neuroscience

Uğur Halıcı

Department of Electrical and Electronics Engineering, Middle East Technical University, Ankara, Turkey

Objective: In the last fifteen years, a new topic called deep learning has attracted researchers in the machine learning community. Artificial neuron model named Perceptron is based on $\mathrm{Mc}$ Culloch and Pitts (1943) neuron model which simply models the operation of neurons in the brain. The Perseptron Convergence Algorithm (1959) that enables training of a single perceptron is a machine learning algorithm proposed in the years when computers were just beginning to develop. With the introduction of the Backpropagation Algorithm $(1975,1987)$, which enables the training of Multi-Layer Neural Networks, there have been significant developments in this field in the 90's. However, the deficiencies in computer capacity and data collection in those years were an obstacle to the development of high performance systems.

Methods: Since the number of parameters increases rapidly when the number of layers is increased in the Multi-layer Perceptron structure, the networks that are trained with a limited number of samples begin to memorize the data instead of developing a generalization ability. By having only a local connections between consecutive layers, instead of the fully connections that is used in the Multilayer Perseptron structure, and also by sharing these local connection weights among the neurons in the same layer, the concept of filters are introduced to the neural networks and so the networks which are are called Convolutional Neural Networks are proposed. The fact that the number of parameters has been reduced significantly in these networks has enabled the use of too many layers and therefore Deep networks have emerged. With the application of the approach called transfer learning, which uses the connection information of the network trained in another field with abundant data, has also enabled a certain success to be achieved in problems with limited data. Developing open source deep learning software libraries that run fast on graphics cards with a large number of processors has brought a significant acceleration to the studies on the subject. Deep Learning continues to evolve very rapidly with Generative Adversarial Networks and Neuromorphic chips, which have become quite important in recent years.

Results and Conclusion: Deep learning applications such as metastasis detection in lymph nodes, object tracking in video, mouse mimic scaling, motor imagery EEG signal classification for brain computer interfaces, airport detection in satellite images are some of the deep learning applications in our METU VISION laboratory.

Keywords: computational neuroscience, artificial neural networks, deep learning

\section{PS8-2}

Model based design of brain-computer interfaces that use code-modulated visual evoked potentials

Yusuf Ziya İder

Bilkent University, Ankara, Turkey

Objective: Code modulated Visual Evoked Potential (cVEP) based Brain-Computer Interfaces (BCI) have high classification speed and accuracy. However, optimal cVEP stimulus design for maximal number of targets and minimal training time is still being studied. The idea behind model-based stimulus design is applying the inverse system model to the EEG response and thus identifying the input. Alternatively, target-specific model predictions are correlated to the observed response. However, even the mass models of the visual system are multi-parametric and nonlinear; e.g. the 17-parameter Robinson model has 4 sigmoidal blocks in addition to many gain and linear filter stages. Parameter identification of this model from real data is not practical. Nagel et.al. have used Moving-Average models to model both the forward and inverse paths, and have developed BCI spellers utilizing both models. However, such linear models need to be validated. Therefore, we have undertaken experiments to determine under what conditions the system obeys superposition.

Methods: Visual stimuli were presented to 7 male subjects on a $60 \mathrm{~Hz}$ monitor. O1, Oz, O2, P3, Pz, P4, P7, P8 EEG channels were recorded using Brain Products V-Amp with 2000sps and were $4 \mathrm{~Hz}-40 \mathrm{~Hz}$ bandpass filtered. Data were reduced to a single signal using Canonical-Correlation-Analysis. Psychtoolbox was used for stimulus presentation on a PC with Ubuntu OS.

Results: EEG response is only sensitive to the edges (Black to White, and White to Black) of the stimulus sequence. The edge responses have $50 \mathrm{~ms}$ delay, and wane within $350 \mathrm{~ms}$. It is shown that responses to certain short stimulus sequences can be accurately predicted by superposition of the edge responses. These atomic sequences are then randomly concatenated to obtain 120 - 
bit long sequences. A 36-target BCI speller application using these yielded classification accuracy and Information Transfer Rate of $95.5 \%$ and $57.19 \mathrm{bits} / \mathrm{min}$, respectively.

Conclusion: Results are promising for further utization of model-based stimulus design paradigms for BCIs.

Keywords: brain-computer interface (BCI), electroencephalogram (EEG), code-modulated visual evoked potential (cVEP), modeling

\section{PS8-3}

Voxelwise modeling for identifying cortical correlates of natural perception and attention

Tolga Çukur ${ }^{1,2}$

'Department of Electrical and Electronics Engineering, Bilkent University, Ankara, Turkey; ${ }^{2}$ National Magnetic Resonance Research Center, Bilkent University, Ankara, Turkey

Traditional neuroimaging paradigms, optimized for hypothesis testing under simplified laboratory settings, have proven to be of limited utility in the study of natural cognition. To address this limitation, we are developing a novel framework that enables the study of brain function during natural behavior. The voxelwise modeling framework starts by recording complex patterns of brain activity during daily-life behavior with functional magnetic resonance imaging (fMRI). We then mine interesting patterns in the recorded data via a forward modeling approach to best brain activity given stimulus and task information. Leveraging this framework, we have tapped into the cortical mechanisms of visual and language representations in the human brain and their modulation by high-level search tasks.

Keywords: computational neuroscience, modeling, perception, attention, natural

\section{PS8-4}

System identification of the sensorimotor transformation in weakly electric fishes

İsmail Uyanık

Department of Electrical and Electronics Engineering, Hacettepe University, Ankara, Turkey

Objective: The behavioral performance of an individual animal is determined via an interplay between its plant and controller. The plant typically includes musculoskeletal components that interact with the environment to generate movement. The controller typically includes sensory systems and neural circuits used to process information to generate motor commands. Our goal is to describe the behavioral and neural representations of the sensorimotor transformation occurring in the controllers of the animals during their free, unconstrained behavior.

Methods: Weakly electric fishes, such as Eigenmannia virescens and Apteronotus leptorhynchus, robustly perform an image stabilization task in which animals track the movement of a refuge in a single linear dimension. We studied refuge tracking in these fishes ( $\mathrm{n}=5$ Eigenmannia and $\mathrm{n}=17$ Apteronotus) using their behavioral responses and chronic tetrode recordings to reveal the dynamics of the sensorimotor activity in the brain.

Results: We identified the frequency response functions of the controllers for each fish using their behavioral responses. Despite the differences in their dynamics, each of these controllers had high-pass filtering characteristics. Moreover, analyzing the chronic tetrode recordings revealed direction-selective neurons, which respond to a range of velocities for the sensory slip, which is the error between the refuge and fish movements. The midbrain electrosensory neurons responded best to high-frequency features, behaving as a high-pass filter on the sensory slip.

Conclusion: The spatiotemporal filtering properties of the midbrain neurons obtained through chronic tetrode recordings matched the system identification results of the behavioral experiments. These results suggest that sensorimotor transformation in the central nervous system pays more attention to high-frequency stimuli rather than the low-frequency content. We believe that this feature plays a critical role behind the high-frequency active sensing movements, which modulates the sensory information in the environment via moving the sensory receptors.

Keywords: electrophysiology, sensorimotor transformation, system identification, weakly electric fishes

\section{Panel 9}

Current Developments in the Neurobiology of Epileptogenesis

\section{PS9-1}

\section{Current developments in the neurobiology of absence} epilepsy

Filiz Onat

Acıbadem Mehmet Ali Aydınlar University, Istanbul, Turkey

Absence epilepsy, characterized by non-convulsive seizures, represents a genetically generalized type of epilepsy that usually begins in childhood or adolescence. Absence epilepsy is characterized by generalized seizures that occur after birth at the end of a latent period called epileptogenesis. The mechanism of this process of epileptogenesis has not been elucidated yet. It has been shown that the cortico-thalamo-cortical loop is important in the pathogenesis of absence epilepsy and in the process of epileptogenesis. There are many experimental genetic and pharmacological models of absence epilepsy. The most commonly used genetic models are rats from Strasbourg (Genetic Absence Epilepsy Rats from Strasbourg; GAERS) and Rijswijk (Wistar Albino Glaxo from Rijswijk; WAG / Rij). There are findings showing that basal ganglia structures are involved in the neurobiological mechanisms of absence epilepsy in GAERS. An existence of an endogenous system associated with substantia nigra pars reticulate is considered to be in the control of spike-and-wave discharges, the characteristic of absence epilepsy seizures. In addition, high amplitude rhythmic synaptic depolarization of striatal projection neurons during spike-and-wave discharges suggests that these neurons also participate in the mechanism of absence 
seizures. There are studies showing that nigro-striatal pathway degeneration has a role in the development of seizures in absence epilepsy rats during epileptogenesis. In this talk, the accumulated data investigating the role of the basal ganglia in the pathopysiology of absence epilepsy will be addressed.

The content has been supported by TUBITAK-SBAG- SBAG218 S653.

Keywords: epilepsy, neurobiology, epileptogenesis

\section{PS9-2}

Sex and age in the neurobiology of epilepsies

Özlem Akman

Department of Physiology, Faculty of Medicine, Demiroğlu Bilim University, Istanbul, Turkey

Brain development, which follows different paths in male and female, involves a series of processes including many important stages such as neurogenesis, gliogenesis, cellular differentiation, migration, synaptogenesis, and myelination. The gonadal steroid hormones exposed at critical periods during prenatal and postnatal development are the main determinants of this sex specific differentiation process. Recent studies show that sex differences in the developmental process may affect the susceptibility to seizures and the progress of epileptogenesis, or may have sex-specific short and long-term consequences of early life seizures. Sex differences in the seizure susceptibility have been shown not only in the developmental period, but also in many studies carried out with adult experimental animals. Sex-related differences in steroid hormones, neurosteroids and neurotransmitter systems, and sexual dimorphism in the structure of the brain including limbic, cortical and subcortical structures that are engaged in the seizure networks may contribute sex differences in certain epilepsy syndromes or susceptibility to seizures. Another important factor affecting the seizure susceptibility and the short and long-term consequences of seizures is age. In this talk, the effects of sex and age in the neurobiology of epilepsies in animal models and the age and sex specific molecular or functional consequences of seizures will be discussed.

Keywords: epilepsy, gender, age

\section{PS9-3}

Antiepileptogenic drug research

Nihan Çarçak

Department of Pharmacology, Istanbul University, Istanbul, Turkey

Epilepsy is a chronic neurological disorder characterized by repetitive seizures that affect many people around the world. Various factors that acutely affect the brain can induce epileptogenesis, a process that results in epilepsy. Despite the efforts in experimental animal models to elucidate the complex mechanisms underlying epileptogenesis and seizure formation in temporal lobe epilepsy, absence epilepsy and other epilepsy syn-

dromes, and even the neuroprotective properties of some antiepileptic drugs have been demonstrated, there is currently no approved treatment option that prevents the development or progression of epilepsy in patients at risk. However, in recent preclinical studies, various intracellular pathways that prevent (antiepileptogenic) or slow-down (modifying) the progression of epilepsy have been enlightened, and novel promising agents acting on these pathways have been identified. More comprehensive neurobiological and clinical studies are needed in this field to ensure that the current laboratory findings are reflected in the epilepsy clinic.

Keywords: epilepsy, drug, treatment

\section{PS9-4}

Spectrum of hyperexcitability of the brain: migraine and pain sensitivity in epilepsy

Sibel K. Velioğlu

Department of Neurology, Faculty of Medicine, Karadeniz Technical University, Trabzon, Turkey

The process that provoke spontaneous seizures that characterizes epilepsy is related to a decrease in the threshold of excitability of neurons. The pathophysiological mechanisms that drive neurons to generate abnormal excessive electrical discharges are still not clearly understood. These neurons, which are silent/calm in the interictal period, are different from normal neurons in many aspects. The conditions that are commonly comorbid with epilepsies, are headaches and painful syndromes. Scientific research on this comorbidity is only limited to clinical studies and has not yet reached results that will clarify the neurobiology of the association. Implementing animal models that have both the disease and the comorbidity and preclinical studies to be performed on them are difficult as methodology, and there is no such study yet. In recent years, two preclinical studies we conducted in experimental animals with genetic epilepsy showed that the pain threshold is reduced in genetic absence epilepsy. Future studies on pain comorbidity in epilepsy will shed a light on the neurobiology of both epilepsy and pain. In this presentation, the neurobiology of epilepsy will be discussed in terms of comorbidity of epilepsy-migraine, and the pain threshold studies in rats with genetic absence epilepsy.

Keywords: epilepsy, migraine, pain

Panel 10

Noninvasive Neuromodulation and Neuroimaging: Customizing Neuromodulation

\section{PS10-1}

Noninvasive neuromodulation and neuroimaging; personalizing the neuromodulation

Lütfü Hanoğlu

Department of Neurology, Faculty of Medicine, Istanbul Medipol University, Istanbul, Turkey 
Objective: Non-invasive neuromodulation (NIBS), especially transcranial magnetic stimulation (TMS) and transcranial direct current stimulation (tDCS), have opened a new therapeutic window as an alternative to pharmacological treatments in the treatment of motor, cognitive and behavioral symptoms of neurodegenerative diseases in recent years. However, while NIBS offers hope for improved treatment modalities, even possible diseasemodifying effects, there is still uncertainty regarding their mechanism of action. Again, in certain cases, it is not possible to solve any real changes in brain functions other than placebo effects or reproducibility problems. As a result, some patients benefit from these treatments in motor, cognitive and behavioral aspects, while others do not. By documenting changes in brain function associated with NIBS applications and using neuroimaging (such as PET, fMR, NIRS, EEG) to guide NIBS administration to achieve a more effective and reliable and specific change in brain function, the procedure appears to be competent and approachable.

Methods: For this purpose, studies have been carried out in order to determine the individuals who can benefit from the treatment in advance, to define the targeted areas individually, to measure the results neurobiologically, to maximize the target effect and to provide a personalized approach as a result.

Results: In this panel, we aimed to review this research area and approach styles within the framework of our findings and different studies conducted with TMS and tDCS and EEG / fMRI in our clinic.

Conclusion: The general framework is presented.

Keywords: noninvasive neuromodulation, neuroimaging, personalized neuromodulation

\section{PS10-2}

Functional and structural imaging of brain networks in determination of non-invasive neuromodulation targets

Zübeyir Bayraktaroğlu

Department of Physiology, International School of Medicine, Istanbul Medipol University, Istanbul, Turkey; Istanbul Medipol University, Regenerative and Restorative Medicine Research Center (REMER), Functional Imaging and Cognitive Affective Neuroscience Laboratory (fINCAN), Istanbul, Turkey

The anatomical substrate of the brain supports the dynamic emergence of the coherent activity in functional networks. The brain regions maintains a specific pattern of structural connections but brain functions are the result of an integrated large scale activity. The brain's systems have features similar to complex networks and the network approaches could provide a powerful way of determining the large scale interactions among brain regions. Although the functional changes occur in shorter time scales, the formation rate of new structural connections is not clear. Nonetheless, strengthening or weakening the connections is possible. Functional loses observed in many neurological and psychiatric disorders can be related to disruption of these connections. Network properties can be used as diagnostic markers and quantify differences between patient and control groups, and many studies have already reported altered brain networks in several patient groups. So that, it is important to understand pathogenesis and treatment of brain disorders from a network perspective. It is also possible that network analysis can be used to follow up the effects of any intervention targeting brain structure and function. In this perspective, it is important to identify physiological or pathological organization of these networks in individuals and how the interventions with the specific nodes of these large scale networks will affect the brain function. However, still there is a long path to travel before to understand whether or not targeting networks nodes is an effective therapeutic tool. We should also learn how to control particular nodes at critical locations within the network that move the brain into specific cognitive states and changing existing connections or creating new ones. We will present examples defining and targeting functional and structural networks from our laboratory.

Keywords: cognitive disorders, functional brain networks, magnetic resonance imaging, neuroimaging, neuromodulation

\section{PS10-3}

Can the possible effect of transcranial magnetic stimulation on cognitive functions in Alzheimer's patients be predicted by EEG classification?

Cihan Bilge Kayasandık ${ }^{1}$, Halil Aziz Velioğlu², Lütfü Hanoğlu

'School of Engineering and Natural Sciences, Istanbul Medipol University, Istanbul, Turkey; 'Istanbul Medipol University, Cognitive Neuroscience, Istanbul, Turkey

Objective: Transcranial magnetic stimulation (TMS) has become widespread in recent years as a treatment alternative for neurodegenerative diseases. Yet, some patients benefit from this treatment while others do not benefit enough. We aim to introduce a classification model for predicting TMS effects on Alzheimer patients by analyzing Electroencephalography (EEG) signals. This is expected to lead personalized TMS treatments.

Methods: We collected spontaneous EEG from patients two weeks before and after TMS therapy (EEG1 and EEG2 respectively). A total of 10 sessions of TMS were performed to the left lateral parietal cortex for two weeks through the instrument of the neuronavigation system. We analyzed the EEG1 signals of 15 Alzheimer patients. We considered the Mini-Mental State Examination (MMSE) scores as a reference to decide whether TMS was a benefit for a particular patient. 7 of the patients were TMS+(MMSE scores increased), 8 of them were TMS-(MMSE scores remained constant). We generated a framework for the prediction of the gain of patients from TMS through analyzing their EEG1 signals. For that purpose, we used Fourier transform for feature extraction, To reduce the dimension, we eliminated $90 \%$ of the coefficients with the small. Then, we applied the Support Vector Machines (SVM) classifier to cluster data into two classes (TMS+andTMS-). Due to the small sample number, we used the leave-one-out method for the validation. We analyzed EEG2 by following the same strategy. 
Results: The average performance of the classification of EEG1 was recorded as $86 \%$. We realized that the EEG2 signals are more informing than the EEG1 to recognize TMS+ and TMSpatients with the $94 \%$ classification accuracy.

Conclusion: We realized that on the analysis of EEG2, SVM assigns larger weights on gamma and beta wavelengths. That concludes that TMS is characterizing the gamma and beta signals in TMS+and TMS- Alzheimer patients.

Keywords: personalized treatments, machine learning, data analysis

\section{PS10-4}

The investigation of the effect of transcranial alternating current stimulation based on individual theta frequency on memory processes with event-related EEG method

Tuba Aktürk ${ }^{1}$, Lütfü Hanoğlu², Bahar Güntekin ${ }^{3}$

'Program of Electroneurophysiology, Vocational School, Istanbul Medipol University, Istanbul, Turkey; 'Department of Neurology, School of Medicine, Istanbul Medipol University, Istanbul, Turkey; ${ }^{3}$ Department of Biophysics, School of Medicine, Istanbul Medipol University, Istanbul, Turkey

Objective: It is known that the neuropsychiatric profile of cognitive impairments varies from person to person, even in the same disease. Therefore, individualized intervention approaches using neuroimaging methods are important. The external manipulation of endogenous brain oscillations with individualized approaches allows us to test the causal relationship, beyond being able to establish a link between brain oscillations and cognitive processes. This study aims to establish a causal relationship between Event-related oscillations and memory processes by individualized theta transcranial Alternating Current Stimulation (tACS) and to modulate memory processes with tACS through this relationship.

Methods: Alpha $(8-13 \mathrm{~Hz})$ and beta (15-28) responses were examined in terms of phase and power characteristics in the timefrequency domain for the preliminary analyzes. 23 healthy young-adult volunteers were included. The study is designed as a single-blind. Participants were randomly divided into placebo $(\mathrm{n}=10)$ and experimental $(\mathrm{n}=13)$ groups. The tACS was applied to the left fronto-parietal area for 20 minutes at a current intensity of $1.5 \mathrm{~mA}$ at a frequency calculated over the individual theta frequency of the individuals. Before and after EEG recordings were performed over 32 channels. During the recording, a visual memory task was presented. At the end of the task, the participants were asked to say which items they remember so that the visual memory scores were obtained.

Results: According to the preliminary findings of the study; In the event-related power spectrum and event-related phase-locking analysis, post-stimulation changes were observed in the experimental group compared to the placebo group, especially in the alpha and beta frequency bands. Also, visual memory scores did not change in the placebo group after the stimulation but increased in the experimental group $(\mathrm{p}<0.05)$.
Conclusion: According to preliminary findings, it is seen that the individualized tACS application has positive effects on visual memory by modulating brain oscillations through different signal features.

Keywords: cognitive neuroscience, event-related oscillation, memory and learning, neuromodulation

\section{Panel 11}

An Interdisciplinary View of Neuromodulatory Systems in Disease and Health

\section{PS11-1}

Investigation of cholinergic effects on principal neurons using optogenetic methods

Çă̆rı Temuçin Ünal

Department of Psychology, Faculty of Arts and Sciences, TED University, Ankara, Turkey

Objective: Cholinergic input to cortical structures arises from basal forebrain cholinergic neurons. In vivo electrophysiological recording and microdialysis experiments have convincingly shown that heighened activity of cholinergic neurons is associated with wakefulness and alert states. The underlying mechanisms of these findings have been investigated using electrical stimulation and pharmacological tools in vitro. This work has shown that cholinergic stimulation is associated with an increased membrane excitability largely through the inactivation of potassium currents in principal neurons. In addition to this, cholinergic stimulation can increase the activity of principal neurons through the activation of a variety of cationic currents in a state-dependent manner. With the advances in optogenetic stimulation methods, the need for verifying the mechanistic explanations of cholinergic stimulation effects on principal neurons has bacome acute. This is especially so for the cholinergic system as cholinergic synapses have been found to be associated with synaptic markers in anatomical studies along with the long known fact that cholinergic neurons in the basal forebrain coexist with noncholinergic neurons.

Methods: Cholinergic neurons of the basal forebrain were optogenetically stimulated in basolateral amygdala slices obtained from adult mice.

Results: In studies using the basolateral amygdalar projection neurons, cholinergic stimulation resulted in decreased membrane excitability, in stark contrast to previous findings. The reduction in excitability seen under these circumstances was reversed in neurons artificially depolarized with intracellular current injections.

Conclusion: These studies have shown the state-dependent nature of cholinergic stimulation effects on principal neurons. In addition, the polarity of the state-dependent effects appear to be difficult to illustrate using more conventional methods that implement pharmacological and electrical stimulation tools. Further studies and theories about cholinergic function will need to take these results into consideration.

Keywords: acetylcholine, principal cell, optogenetics 
PS11-2

Neural circuits impacted by dopamine loss

Bengi Ünal

Faculty of Arts and Sciences, Department of Psychology, TED University, Ankara, Turkey

Objective: Progressive degeneration in dopaminergic cells is the hallmark of Parkinson's disease (PD). Dopaminergic loss particularly impacts the striatum, the main input domain of the basal ganglia; and causes an imbalance in the recruitment of the direct and indirect pathways emanating from the striatum.As a result, the rest of the basal ganglia neural communication gets hampered, and this gives rise to motor problems including difficulties with the initiation of volitional movements and appearance of abnormal involuntary behaviors. The fact that PD diagnosis is usually possible at the later stages of dopaminergic degeneration suggests that the neural circuit targeted by the dopaminergic system can show certain adaptations that can buffer the progressive dopamine loss up to a degree. Determining neural components that are capable of these changes may confer benefits in the future for the early diagnosis and effective treatment of PD.

Methods: In the tyrosine hydroxylase (TH)-Cre expressing mice, PD is modeled by injecting dopamine specific neurotoxin, 6-OHDA, unilaterally into midbrain dopaminergic cells. Subsequently, the mice were divided into two groups; and one of the groups received an intrastriatal injection of AAV2-flextaCasp3-TEVp virus to able TH-expressing GABAergic striatal interneurons. In mice, motor behaviors following striatal dopaminergic loss and motor behaviors in response to increasing L-Dopa dosages were monitored and quantified.

Results: In PD-modelled mice, in which TH-Cre expressing GABAergic local circuit neurons were virally ablated in the dopamine depleted striatum, progressive treatment with increasing dosages of L-Dopa triggered a lesser degree of abnormal involuntary movement movements compared to other groups.

Conclusion: These results suggest that, TH-Cre expressing GABAergic interneurons are important cellular components of the neural circuits undergoing cellular and synaptic adaptations due to dopamine loss. To increase the efficacy of widely utilized dopamine replacement therapies by L-DOPA and lessen its side effects, such cell type- and synapse- specific changes should be taken considered.

Keywords: dopamine, Parkinson's disease, striatum, behavior

\section{PS11-3}

A new neuromodulator pathway: basal forebrain gabaergic innervation of the limbic circuits

Güneş Ünal

Department of Psychology, Behavioral Neuroscience Laboratory, Boğaziçi University, Istanbul, Turkey

Objective: Neuromodulator projections arising from basal forebrain and brainstem target limbic regions and the cerebral cortex to regulate several cognitive and affective functions. The basal forebrain, with its well-known role in memory processes, provides dense GABAergic input to several regions, in addition to its cholinergic projections. Septo-hippocampal GABAergic neurons of the basal forebrain regulate hippocampal theta oscillation and contribute to resulting memory processes. This study is based on the theory that basal forebrain GABAergic projections form a general neuromodulator pathway by carrying out similar functions in their target regions. It aims to characterize ventral pallidum and substantia innominata GABAergic neurons targeting the amygdala and the bed nucleus of stria terminalis.

Methods: Long-range neurons targeting the basolateral complex and the extended amygdala from different basal forebrain nuclei are revealed by retrograde tracing. Different subnuclei of the amygdala and the BNST are targeted in adult Wistar rats under stereotaxic surgery. In each animal, two specific subnuclei are unilaterally injected with different color fluorescence tracers (RetroBead, Lumafluor). Neurochemical profiles of the traced neuros are revealed by immunohistochemistry. Putative GABAergic neurons are categorized into subgroups according to the biomarker molecules they express.

Results: We identified non-cholinergic neurons in the ventral pallidum/substantia innominata nuclei of the basal forebrain that target the lateral nucleus of the amygdala and the central subdivision of the BNST. Utilizing somatic staining, we revealed that these putative GABAergic or glutamatergic neurons are proportionate to the cholinergic neurons in the ventral pallidum. We identified a non-cholinergic, calbindin-immunopositive subgroup of putative GABAergic neurons that mainly target the ventral subregions of the lateral nucleus of amygdala.

Conclusion: These results reveal neurochemical subgroups of GABAergic basal forebrain-amygdala neurons that have previously been identified by anterograde tracing. This finding indicates that basal forebrain GABAergic neurons targeting the amygdala and the extended amygdala are structurally similar to the septo-hippocampal GABAergic pathway.

Keywords: basal forebrain, GABA, amygdala, BNST, retrograde tracing

\section{PS11-4}

Reward prediction error and phasic changes in striatal dopamine concentration associated with reward proximity

Mehmet Kocatürk

Department of Biomedical Engineering, Istanbul Medipol University, Istanbul, Turkey

Objective: Many neurological and neuropsychiatric disorders such as Parkinson's disease, schizophrenia, and substance abuse are associated with disruptions or damages in the dopamine system. Therefore, the dopamine system is currently an active research topic. In vivo studies performed with electrophysiological methods revealed that dopamine neurons fires continuously at low frequency and occasionally at high frequency during reinforcement learning. The findings showed that dopamine neurons encode the difference between magnitudes 
of the expected and received rewards, in other words reward prediction error (RPE).

Methods: Fast-scan cyclic voltammetry (FSCV) enables realtime detection of dopamine release in the structures innervated by dopaminergic axons. Two male Wistar rats were chronically implanted with carbon fiber microsensors in the left ventromedial striatum and dopamine concentration changes were measured using FSCV during behavior. Rewards (sucrose solution) were presented unexpectedly and during instrumental conditioning. The rats are then conditioned to initiate the motion of a robotic actuator carrying reward towards them.

Results: Based on the recordings performed using two rats, we detected the reward given during instrumental conditioning caused a higher dopamine concentration increase compared to the reward delivered unexpectedly $(\mathrm{p}<0.0005)$. We monitored that the changes in the dopamine concentration were associated with the spatial proximity of the reward, which was brought to the rat by a robotic actuator through varying trajectories.

Conclusion: The results indicate that the rewards given following reward-motivated behavior leads to a higher dopamine release compared to the rewards delivered unexpectedly, and the changes in the spatial proximity of the reward cue (robotic actuator) causes fluctuations in the dopamine concentration in the ventromedial striatum. These findings suggest that the initiation of a reward-motivated trial and the proximity of the reward to the subject can be predicted by monitoring the dopamine concentration.

Support: The Scientific and Technological Research Council of Turkey (TÜBİTAK), Grant No: EEEAG-117E286

Keywords: dopamine, reinforcement learning, electrophysiology, voltammetry

\section{Panel 12}

Neurobiological Bases of Metacognition: EEG / ERP Findings

\section{PS12-1}

\section{Comparing temporal dynamics of retrospective and prospective metacognitive judgments}

\section{Metehan Irak ${ }^{1}$, Can Soylu ${ }^{1}$, Berna Güler ${ }^{1}$, Berkhan Karslı ${ }^{2}$}

'Department of Psychology, Bahçeşehir Unıversity, Istanbul, Turkey; 'Ludwig Maximilian University of Munich, Neuro-Cognitive Psychology Program, Munich, Germany

Objective: Metacognition is defined as cognition about cognition, knowing about knowing, the ability to reflect on, monitor and control other cognitive processes. Metacognitive judgments can be retrospective (retrospective confidence judgements: RCJs) or prospective (feeling of knowing: FOK). FOK is one such metacognitive process which allows individuals to make predictions about the likelihood of their remembering in the future. RCJ, on the other hand, refers to a subjective evaluation of the confidence in a previous decision (after recall or recognition). It is still controversial whether FOK relies on a domain-general (shares resources across many situations) or domain specific (single underlying resource).

Methods: A total 85 undergraduate students (51 females; Mage=21.18) were participated. Word-pair list was used for episodic memory task, on the other hand general information questions were used for semantic memory and, and for both task classical recall-judgment-recognition paradigm was used. Stimulus presentation, recording, storage, and analysis were carried out using a 32 channel EEG/EP NeuroScan system. ERPs were analyzed at frontal, central, and parietal regions at different time windows.

Results: A 2 (Judgment type: FOK and RCJ) x3 (Hemisphere: Frontal, central and parietal) $\mathrm{x} 3$ (Region: right, center and left) repeated measure MANOVA was used to analyze the data. Behavioral results indicated that FOK judgments support domain specific hypothesis, whereas RCJs are domain general process. Although FOK and RCJs produced similar ERP waveform, amplitude values of these judgments were different withintask, across memory tasks, at different time windows and different regions, $\mathrm{F} \geq 3.49$, $\mathrm{p} \leq .04$.

Conclusion: We concluded that metacognitive judgments are related to gradient neural circuits that are shared and spread to the brain, and this situation may also vary depending on the type of memory process.

Keywords: episodic memory, event-related potentials, metacognitive judgments, semantic memory

\section{PS12-2}

Comparison of electrophysiological correlation of judgment of learning and feeling of knowing during face-name recognition

Metehan Irak ${ }^{1}, \underline{\text { Can Soylu }}{ }^{1}$, Gözem Turan², Berna Güler ${ }^{1}$

'Department of Psychology, Bahçeşehir University, Istanbul, Turkey; 2Institute of Psychology, Goethe-Universität, Frankfurt, Germany

Objective: Purpose of this study was to investigate and compare the neural correlates of judgment of learning (JOL) and feeling of knowing (FOK) as ERPs during face-name recognition (FNR).

Methods: 74 healthy university students (45 women, 29 men; Mage $=21.15, \mathrm{SD}=1.47$ ) were participated to the study. The experiment consists of six successive stages as follows; learning (face), learning (face-name), JOL, recognition, FOK and recognition. Stimulus presentation, recording, storage, and analysis were carried out using a 32 channel EEG/EP NeuroScan 4.5 system. EEG recordings were carried out in a room isolated from noise and electrical field, and these activities were recorded in international 10-20 system from 30 electrodes.

Results: During FNR processing, FOK and JOL indicated significant differences in early (N100,N170 and P100), middle (N200, P200 and P300) and late (300-700 ms) time windows in different electrode sides. Even though JOL and FOK judgments produced similar ERP waveforms, temporal dynamics of these 
two judgments were different. Levels of FOK (high and low) were associated with amplitude values in N100, P100, P200 and P300; and high FOK judgments indicated higher amplitude values compared to low FOK judgments. On the other hand, levels of JOL were only linked to amplitude values in N170; and high JOL were lower in amplitude values compared to low JOL decisions.

Conclusion: To conclude, results of the present study indicated that amplitudes values in early and middle-late time windows were differentiated according to types of metacognitive decisions (JOL and FOK) and their levels (high and low).

Keywords: judgment of learning, feeling of knowing, face-name recognition, ERP

\section{PS12-3}

Effects of worry on feeling of knowing judgments: an event-related potentials study

Berkhan Karsl ${ }^{1}$, Mustafa Yildız ${ }^{2}$, Can Soylu' ${ }^{2}$, Metehan Irak $^{2}$

'Department of Psychology, Ludwig Maximilian University of Munich, Munich, Germany; '2Department of Psychology, Bahçeşehir University, Istanbul, Turkey

Objective: Worry, as a cognitive component of anxiety, has a significant influence on metamemory and metacognitive judgments. Worry is a psychological concept and it is a form of thinking about the future. During the worry which consists of thoughts that are not pleasant and comfortable, people feel that the situation cannot be controlled consciously by trying to direct attention to other things. Feeling of knowing (FOK) is one such metacognitive process that allows individuals to make predictions about the likelihood of their remembering, in future, information that cannot be currently recalled. Previous behavioral studies regarding the effects of worry on metacognitive judgments are inconsistent. In addition, the temporal dynamics of worry on metacognitive judgments are unknown. Here we investigated the effect of worry on ERP correlates of FOK judgments.

Methods: Neural correlates of FOK were induced by a wordpair list using classical episodic recall-FOK judgment-recognition paradigm. A total of 20 university students (11 female; Mage $=21.45)$ was divided into two groups namely low $(n=13)$ and high worry ( $\mathrm{n}=7)$ according to Penn State Worry Questionnaire (PSWQ). Stimulus presentation, recording, storage, and analysis were carried out using a 64 channel EEG/EP NeuroScan system.

Results: It was detected that participants with high worry had lower FOK accuracy compared to low worry. We also observed that ERP correlates of FOK judgments differ according to high versus low worry.

Conclusion: Findings were discussed in terms of the cognitive and neurobiological implications of the effect of worry on metacognitive judgments and decision-making processes in clinical and healthy samples.

Keywords: worry, ECG, ERP, FOK judgments, metamemory

\section{Panel 13}

Doctoral Programs in Neuroscience:

Differences, Shortcomings, Advantages, Disadvantages, Solutions

\section{PS13-1}

The PhD programs in neuroscience: differences, deficiencies, advantages, disadvantages, solutions

Esat Adigüzel

Department of Neuroscience, Institute of Health Sciences, Pamukkale University, Denizli, Turkey

Objective: As scientists' interest in neuroscience increases, neuroscience $\mathrm{PhD}$ programs are becoming widespread in our country. Neuroscience, which is a combination of many disciplines, encourages scientists from different fields to collaborate.

Methods: However, knowledge level of students who just started studying Neuroscience at phd level and do not have any educational background regarding the topic, create problems that need to be discussed. It is difficult to provide an education that includes all fields of neuroscience in one phd program. In addition to that, it is not possible for a university to have academician trained in all scientific fields related to neuroscience.

Results: The objectives of this panel include the following topics: To reveal the problems that may arise from the issues mentioned above. To discuss the approach differences among universities in neuroscience phd programs.

Conclusion: To propose solutions to issues that are analysed as a problem in neuroscience phd programs.

Keywords: neuroscience, $\mathrm{PhD}$, education

\section{PS13-2}

The Doctoral Program at Hacettepe University Neurological Sciences and Psychiatry Institute

Turgay Dalkara

The Institute of Neurological Sciences and Psychiatry, Hacettepe University, Ankara, Turkey

The motto for the establishment of HUINP is to create a common platform providing the infrastructure for intensive collaboration between basic and clinical sciences in order to carry out translational research in the field of neurological and psychiatric sciences. This pioneering vision, which started to be implemented in 1982, saw the $\mathrm{PhD}$ training of neurology, psychiatry and neurosurgery specialists in the field of neuroscience as an essential tool to achieve this goal. The success achieved in this goal led to integration the $\mathrm{MD} \mathrm{PhD}$ program into the Institute structure. In this context, 50 specialists and 20 medical students have been given the opportunity to do a doctorate so far. This powerful researcher capacity is complemented with 
an integrative laboratory infrastructure that includes in vitro and molecular research facilities, in vivo wild type and transgenic animal models, behavioral set-ups, advanced imaging techniques and, optogenetic and optic approaches. HUINP being located in the same venue with a modern University hospital having a rich patient capacity and the most advanced infrastructure in Turkey has created a favorable environment for research from bench to bed side. This pioneering vision and experience, which is one of the firsts in the world has made HUINP an internationally recognized attraction center for translational research. Many visiting researchers from European and other countries have found this structure very instrumental to further their experience in translational neuroscience research. Another feature of HUINP is its intense cooperation with the leading international centers. This experience increases the recognition of our faculty members, enables our graduates to easily find positions in these centers and creates opportunities for visiting postdocs to continue their careers.

Keywords: $\mathrm{PhD}$, program, neuroscience

\section{PS13-3}

Significance of multidisciplinary approach in neuroscience education

Erdem Tüzün

Department of Neuroscience, Faculty of Medicine, Istanbul University, Istanbul, Turkey

Objective: A comprehensive understanding of neuroscience seems possible by prioritizing diversity in both researchers and research issues. Accordingly, the aim of doctoral programs in neuroscience is to prepare students for an interdisciplinary and collaborative global academic environment. It is extremely important to be able to prepare graduates for interdisciplinary thinking and study planning. However, it is equally critical to provide students with rich research experience in a particular subject in doctoral education. The goal should be to provide students with a wide range of experience in the subjects they prefer to specialize in early in their academic life. In this way, researchers who have knowledge of certain research methods in the field may be trained.

Methods: To achieve this goal, universities should design their doctoral programs that will demonstrate their strengths in different fields of neuroscience. The doctorate program conducted by Istanbul University Aziz Sancar Institute of Experimental Research, Neuroscience Department focuses on molecular and cognitive neuroscience studies. The students learn basic neuroscience concepts, how neuroscience research is carried out, and current changes in various neuroscientific disciplines in compulsory courses. The elective courses give option for them to focus on the areas they want to specialize in.

Results: In doctoral programs, the aim should be to limit the students in certain scientific areas. Simply designing a doctorate program on paper should not mean that everything will go well. If you want to train a PhD student as a scientist, you have to set him/her free.

Conclusion: In summary, the rapidly increasing knowledge in neuroscience requires doctoral students to learn many information and techniques. In this case, students' desire to transfer their intellectual energies to many different disciplines may result in compromising the deepening of the technical skill to be learned.

Keywords: neuroscience, education, multidisciplinary

\section{PS13-4 \\ Now, it is time to be brave to bring what is distant nearby \\ Neslihan Serap Şengör \\ Istanbul Technical University, Istanbul, Turkey}

Objective: Neuroscience, beyond being interdisciplinary, is a field of science where the tools, methods and approaches of life sciences, social sciences and formal sciences are functional all together. Today, higher education has been reduced to gaining the ability to use tools and techniques in certain subjects. The perspective and skills gained by undergraduate education is far from comprehending the philosophy of knowledge in an interdisciplinary area. Teaching only methods and techniques is insufficient to have the basic knowledge required to be competent in neuroscience which requires different scientific perspectives. The aim of this talk is to discuss what should be done while designing the curriculum for graduate studies in neuroscience.

Methods: First, the graduate curricula of different universities will be briefly mentioned, and how different programs can be designed will be shown and the diversity of studies in neuroscience will be pointed out.

Results: The graduate neuroscience programs in Turkey generally mostly prefer considering the sub-discipline in medicine being far from a synthesis of different sciences. Even when different disciplines are considered they are mostly considered to introduce some methods that would be useful as tools. However, for the application to associate professorships, even under the engineering branch, the title of neuroscience is included; and even in engineering programs, neuroscience courses are opened and theses are carried out. Just as considering neuroscience programs dominantly one-sided in medicine, it is unwise to conduct such studies away from the field of medicine in engineering. Maybe we need to leave our comfort areas and take a step towards each other.

Conclusion: In this speech, a way of constructing a common language will be suggested and it will be emphasized that it is possible to create highly integrated neuroscience programs in virtual environments, rendering physical distances nearby with the new skills that that are obtained nowadays.

Keywords: Life sciences, formal sciences, on-line learning 
PS13-5

\section{Introduction to Ege University Neuroscience Department and overview of neuroscience PhD programs}

Lütfiye Kanıt

Department of Neuroscience, Institute of Health Sciences, Ege University, Izmir, Turkey

Objective: Neuroscience Department at Ege University was established in 2010 with a multidisciplinary structure, affiliated to the Institute of Health Sciences and started to accept students for M.Sc. and PhD since 2011. Currently, 12 M.Sc. students and 20 $\mathrm{PhD}$ students are enrolled at the program. So far, twenty-three M.Sc. and two $\mathrm{PhD}$ students have successfully graduated this program.

Methods: In the last 5-10 years, throughout the world, especially in Europe, doctoral education has begun to move away from the focus of the department, and project-based doctoral training has started to become increasingly common. In project-based doctoral programs, the number of compulsory courses students take is either very little or not at all. Additionally, applications for these doctoral programs do not generally require a bachelor's or master's degree in a specific field.

Results: For instance, a student who has studied mathematics and has a master's degree in philosophy can easily apply to a neuroscience doctoral program. This approach supports the multidisciplinary perspective and breaks the patterns of the department, providing researchers with the freedom to work individually. Although graduates of these programs have a high potential to do research and produce publications, their education in basic science subjects may be weak.

Conclusion: In our country, there are big differences between the centers that provide neuroscience doctorate education in terms of their focus on the education and field of study. We need to see this as a wealth, not a deficiency. Because we can offer many students the opportunity to work and learn in the field they want. The important point here is to enable students to consciously choose the centers they want to get their doctorate degree. In order to achieve this, the research facilities of each program should provide the graduate theses and publications contributed by the graduates on their WEB pages.

Keywords: $\mathrm{PhD}$ education, neuroscience department, projectbased $\mathrm{PhD}$ training

\section{PS13-6}

Interdisciplinary education in neuroscience: Bilkent example

Hulusi Kafalıgönül

Interdisciplinary Neuroscience Program, Aysel Sabuncu Brain Research Center, Bilkent University, Ankara, Turkey

Objective: As the global challenges for humanity become more diverse and difficult, the importance of multidisciplinary and interdisciplinary research has increased, and the scientists having such perspective have become an important factor. Similarly, there is a growing realization of this approach in the field of Neuroscience. Broadly speaking, although the basic principles and guidelines for interdisciplinary education in neuroscience are not concrete and clear yet, significant progress has been made along this direction.

Methods: Bilkent University - Interdisciplinary Neuroscience Program, is one of the first neuroscience programs in our country established with an interdisciplinary perspective. Providing specific examples from this education program, the requirements, advantages, and difficulties of interdisciplinary approach for neuroscience education will be discussed.

Results: Following certain training principles what kind of achievements can be made and their long-term effects will be highlighted. Likewise, the main problems encountered, possible solutions, and alternatives will be listed and some reflections will be presented.

Conclusion: In the last part of the panel presentation, the importance and complementary role of research-oriented educational activities in the field of interdisciplinary neuroscience will be emphasized. Moreover, brief information will be given about what kind of neuroscience activities across the country and universities can directly contribute to graduate education, and which steps can be taken to spread these educational activities will be presented. Obviously, one of the most important factors in graduate education is the research environment in the department and laboratories. In this scale, what kind of educational activities and principles can be carried out by advisors/mentors will be presented. Regular practice of these activities can lead to important differences in the long-term and some prior examples will be provided in this respect.

Keywords: interdisciplinary, multidisciplinary, education, neuroscience

\section{PS13-7}

Neuroscience and neurotechnology education: METUHacettepe University joint doctoral frogram example

Uğur Halıcı

Electrical and Electronics Engineering, METU, Ankara, Turkey

Objective: Neuroscience (Neuroscience) is the field of science that studies the nervous system. The areas of life sciences such as cellular and molecular biology, physiology and anatomy, science and engineering fields such as physics, chemistry, electricity and electronics, computer science; It includes interdisciplinary studies conducted in a wide range of fields including clinical medicine such as neurology and psychiatry, as well as cognitive sciences, psychology and social sciences. Neurotechnology (Neural Engineering) aims to develop technologies that, facilitate the diagnosis and treatment of neural diseases or help compensate for damaged nervous system functions, as well as the development of brain-inspired software and hardware technologies that 
bring innovative solutions in the field of computers. There are many difficulties in providing training in such a wide range of subjects. In this panel on Neuroscience and Neurotechnology Education, the challenges in this field of education and the approaches that can be used to overcome it will be discussed.

Methods: Neuroscience and Neurotechnology PhD program brings together two universities, METU, Graduate School of Natural and Applied Sciences and HU, Institute of Neurological Sciences and Psychiatry, in order to support joint research between basic/clinical neurologic sciences and related technologies.Through this Doctoral Program, the needs, advantages and challenges of Neurotechnology or Neural Engineering education will be discussed with specific examples.

Results: Students of doctoral programs usually come from the field of Medicine or Engineering. There are many difficulties for students with medical or engineering backgrounds to start in Neuroscience and Neurotechnology at the doctoral level.

Conclusion: Study in neuroscience, and especially Neurotechnology, also known as Neural Engineering, should begin at the undergraduate level. It is essential to train students who are equipped with competent knowledge in two fields, by providing mathematics and engineering education together with the knowledge about biology and medicine from the beginning.

Keywords: neuroscience, neurotechnology, neuroenginnering, education

\section{Panel 14}

\section{Electrophysiological Markers in Cognition}

\section{PS14-1}

\section{Electrophysiological markers in cognition}

Canan Kalaycıŏglu

Department of Physiology, Faculty of Medicine, Ankara University, Ankara, Turkey

Objective: Cognition is the process of knowing. It includes brain processes related to acquiring knowledge, learning, understanding and reasoning. Cognition requires higher brain functions. Attention -which is essential for focusing stimuli; language -which is important for communication; learning -which provides to take in new things and integrate them with prior knowledge; memory -which is necessary for coding, storing and retrieving information; perception -which allows to take information through sensory systems and to respond it; can be given as examples of cognitive processes. These are fundamentally the function of the cortex, but the contribution of subcortical areas is necessary.

Methods: EEG enables the investigation of electrical activity from cortical neurons at high temporal resolution. It is inexpensive and non-invasive. Brain functions occur through anatomical-functional connections between neurons. EEG signal analysis methods provide information about the activity of these connections. Recordings during experimental conditions such as sensorimotor and cognitive events, sleep and wakeful- ness are useful to investigate cognitive processes both in healthy and patient groups. When endogenous and exogenous stimuli trigger sensorimotor and cognitive processing evoked potentials and event-related potentials occur. These potentials are time-locked to the stimulus and originate from the synchronous oscillatory activity of neuronal ensembles. To investigate the oscillatory activity, digital filtering is applied to the evoked and event-related potentials.

Results: A biomarker is a characteristic that is measured as an indicator of normal biologic processes, pathogenic processes, or responses to an intervention (such as therapeutic interventions). Biomarkers must be highly specific and sensitive. False positive and negative indicator rates are expected to be low. Biomarkers may have molecular, histologic, radiographic, or electrophysiological etc. characteristics.

Conclusion: There are many researches showing the existence of specific EEG changes in cognitive processes either healthy people or many diseases. The results support that EEG data can be used as biomarkers.

Keywords: electrophysiological biomarkers, EEG, cognition

\section{PS14-2}

Are sleep spindles electrophysiological marker of cognition?

Nakşidil Yazıhan

Department of Psychology, Çankaya University, Ankara, Turkey

Sleep spindles, which are one of the microstructural graph elements of EEG appearing in sleep, have attracted steadily increasing attention in recent years. Sleep spindles, as one of the defining features of second stage of Non-REM sleep, are phasic bursts of thalamocortical activity which typically defined with a frequency ranging between $11-16 \mathrm{~Hz}$ (in the sigma frequency band) and a duration between $0.5-2.0$ seconds. While the sleep spindles show an unchanging feature from night to night in an individual, significant differences can be observed between individuals (De Gennaro\&Ferraro, 2003). Functionally, sleep spindles are involved in the processes of offline memory consolidation (Schabus et al., 2004). The consolidation of declarative memory results from a dialogue between reactivated hippocampal memory traces and neocortical networks that retain long-term memory representations. Sleep spindles are the key components for the stabilization of memory traces at night with the synchronized activity of hippocampal ripple waves and slow waves (Dudai, Karni \& Born, 2015). In short, sleep spindles facilitate neuroplasticity and off-line processing during sleep, and supports learning, memory consolidation, and other cognitive functions. Numerous studies have been published investigating the association between sleep spindles and cognitive functions for the last 20 years. This talk will focus on the characteristics of sleep spindles and the association of these oscillations with cognitive abilities. Moreover, it will focus on sleep spindle changes among different populations such as schizophrenia.

Keywords: sleep spindle, memory consolidation, learning, sleep 


\section{PS14-3}

Formation of functional cognitive networks: theta oscillations and synchrony

Simge Aykan

Department of Physiology, School of Medicine, Ankara University, Ankara, Turkey

Objective: Functional neural networks are formed by the activation of the connections of the neural ensembles during a function. In this way, interconnected neurons provide the necessary infrastructure for the functional properties of the brain. Besides being anatomically connected, this organization enables the establishment of flexible functional networks in and between the cortical regions by showing synchronous or asynchronous activation.

Methods: Cortical oscillations occur with the simultaneous activation of neural ensembles. Oscillations are an effective mechanism for combining regional neuron groups into wider networks. Networks formed by specific neural ensembles in the brain underlie all kinds of cognitive activity. Synchronization in each of the traditional EEG frequency bands (delta, theta, alpha, beta, gamma) facilitates different cognitive functions.

Results and Conclusion: Theta band plays a role in the transmission of information between distant regions and in controlling information processing locally with theta-gamma coupling. Theta generally provides communication between the hippocampus and prefrontal cortex and plays a role in memory functions. It is also believed to be involved in coordinating neurons. This talk will focus on how functional connectivity networks are coordinated with theta oscillations. Theta oscillations will be discussed in terms of episodic memory and spatial learning functions. Theta oscillation changes in neurodegenerative and neurodevelopmental diseases will be discussed.

Keywords: cognitive networks, episodic memory, theta oscillations

\section{PS14-4}

\section{Event related potentials in language processing}

Seren Düzenli Öztürk

Department of Speech and Language Therapy, Faculty of Health Sciences, Izmir Bakırçay University, Izmir, Turkey

Language, which is regarded as one of the distinctive characteristics of humans, has attracted interest of researchers from the field of neuroscience for a long time. Language is a system that has being used to understand and express emotions and thoughts by means of arbitrary symbols. The integrated five main components of language are syntax, semantics, phonology, morphology, and pragmatics. As a non-invasive method, event-related potentials are one of the electrophysiological methods that reflect information processing and being obtained with high temporal resolution. This talk will focus on language related potentials such as Mismatch Negativity
(MMN), N400, and P600. The characteristics of these potentials will be discussed briefly with the cases of language disorders, schizophrenia, aphasia, dementia, bilingualism in the context of language acquisition process. In addition, within this framework, recent findings of our research group on eventrelated potentials modulated by Turkish word semantic processing in healthy participants will be summarized.

Keywords: language, event related potentials, mismatch negativity (MMN), N400, P600

\section{Panel 15}

The Roles of the Image, Prediction and Duration in Visual Perception

\section{PS15-1}

Expectations, predictions, prior knowledge and visual perception

Hüseyin Boyacı

Department of Psychology, Bilkent University, Ankara, Turkey

Objective: Expectations, predictions and prior knowledge affect our perception, and under certain conditions even determine what we perceive.

Methods: Then how do expectations, predictions and prior knowledge form in the visual system?

Results: Where and how are they coded, and how do they affect the function of the system?

Conclusion: How can their effects be modeled mathematically at the behavioral and neuronal level? In this presentation these topics will be covered and examples will be presented.

Keywords: expectation, prediction, prior knowledge, mathematical modelling

\section{PS15-2}

Stimulus motion- and motor action-induced prediction mechanisms in the visual system

İnci Ayhan $^{1}$, Didem Alaşhan ${ }^{2}$, Dilce Tanrıverdi ${ }^{1}$, Mehmet Yörüten ${ }^{1}$

'Department of Psychology, Faculty of Arts and Sciences, Boğaziçi University, Istanbul, Turkey; Institute for Graduate Studies in Social Sciences, Cognitive Science Program, Boğaziçi University, Istanbul, Turkey

Objective: Roach et al (2011) suggested that as well as analyzing image velocity, motion-sensitive neurons in cortex might also predict future patterns of sensory stimuli along the motion path: Locally drifting sinusoidal target patterns presented at the leading edge of a region containing phase-congruent motion can be detected at very low contrasts, indicating a reduction in discrimination thresholds in comparison to those obtained in target-phase-incongruent motion conditions. Roach et al studied predictive mechanisms while eyes were 
steady. Here, we aim to investigate prediction of spatio-temporal patterns in the presence of smooth pursuit eye movements as well as stimulus motion.

Methods: Experiments were conducted on 4 observers using psychophysical metholodologies. Roach et al.'s paradigm was changed as to involve smooth pursuit eye movements. 3 independent variables were: (1) Phase congruency between the inducer and target motion (0-180) (2) Target position relative to the direction of inducer motion (trailing-leading) (3) Target position relative to the direction of eye movement (trailing-leading).

Results: In eye-steady blocks, the main effect of phase modulation was statistically significant such that the contrast thresholds in phase-congruent condition were lower than those in phaseincongruent condition, $\mathrm{F}(1.3)=122.54, \mathrm{p}=0.002$. In dynamiceye/static-inducer-and-target blocks, phase modulation was significant only in conditions, where the target was in trailing position relative to the direction of eye movement, $\mathrm{F}(1.3)=20.026$, $\mathrm{p}=0.02$. In the presence of both stimulus motion and eye movements, the main effect of phase was again significant, $\mathrm{F}(1.3)=16.57 \mathrm{p}=0.027$, thresholds in $0-180$ conditions being different only if the target was in leading position relative to the direction of both inducer motion and eye movement.

Conclusion: Spatial pattern prediction can be triggered by smooth pursuit eye movements, as well as stimulus motion and that these two different effects may interact. Acknowledgment: Tübitak 1001 Grant Number 218K282.

Keywords: sensorimotor interaction, prediction, vision, motion perception

\section{PS15-3}

\section{Seeing through transparent layers}

Dicle N. Dövencioğlu ${ }^{1}$, Andrea Van Doorn ${ }^{2,3}$, Jan Koenderink ${ }^{2,3}$, Katja Doerschner ${ }^{4,5}$

'Department of Psychology, Middle East Technical University, Ankara, Turkey; ${ }^{2} \mathrm{KU}$ Leuven, Leuven, Belgium; ${ }^{3}$ Utrecht University, Utrecht, The Netherlands; 4Justus-Liebig-University Giessen, Giessen, Germany; ${ }^{5}$ Department of Psychology, Bilkent University, Ankara, Turkey
Objective: The human visual system is remarkably good at decomposing local and global deformations in the flow of visual information into different perceptual layers. It is critical as we can effectively drive through heavy fog or hard rain; or decide whether an object in a river is animate while fishing. In such complex scenes, changes in visual information might be due to observer motion, object motion, deformations of the transparent medium (such as the atmospheric events), or a combination of these. How does the visual system attribute motion information to certain layers?

Methods: We used eidolons to investigate equivalence classes for perceptually similar transparent layers. We created a stimulus space for perceptual equivalents of a fiducial scene by systematically varying the local disarray parameters reach and grain. This disarray in eidolon space leads to distinct impressions of transparency, specifically, high reach and grain values vividly resemble water whereas smaller grain values appear diffuse like structured glass. We asked observers to adjust image deformations so that the objects in the scene looked like they were seen (a) under water, (b) behind haze, or (c) behind structured glass. Observers adjusted image deformation parameters by moving the mouse horizontally (grain) and vertically (reach).

Results: For two conditions, water and glass, we observed high intraobserver consistency: responses were not random. Responses yielded a concentrated equivalence class for water and structured glass. By using two basic image parameters, we show that it is possible to describe image information as rich as complex transparent layering.

Conclusion: Transparent mediums can cause similar deformations while being different in their nature. We have a rich lexicon to describe the nature of transparent layers, here we show the image properties that identify some typical transparent layers, specifically the equivalence classes of water, glass, and haze.

Keywords: visual perception, image parameters, transparency, water, glass 


\section{Oral Presentations}

\section{(SS1 - SS3)}

\section{SS1-A}

Molecular and Cellular Neuroscience

\section{SS1-A01}

Environmental enrichment alters synaptic dynamics in an age-specific manner in zebrafish

Elif Tuğce Karoğlu Eravşar ${ }^{1}$, Melek Umay Tüz Şaşik², Michelle Adams ${ }^{3}$

'Interdisciplinary Program in Neuroscience, Aysel Sabuncu Brain Research Center, Bilkent University, Ankara, Turkey; National Nanotechnology Research Center (UNAM), Bilkent University, Ankara, Turkey; Molecular Biology and Genetics Zebrafish Facility, Bilkent University, Ankara, Turkey; National Magnetic Resonance Research Center (UMRAM), Bilkent University, Ankara, Turkey; Department of Psychology, Selcuk University, Konya, Turkey; ${ }^{2}$ Interdisciplinary Program in Neuroscience, Aysel Sabuncu Brain Research Center, Bilkent University, Ankara, Turkey; National Nanotechnology Research Center (UNAM), Bilkent University, Ankara, Turkey; Molecular Biology and Genetics Zebrafish Facility, Bilkent University, Ankara, Turkey; National Magnetic Resonance Research Center (UMRAM), Bilkent University, Ankara, Turkey; ${ }^{3}$ nterdisciplinary Program in Neuroscience, Aysel Sabuncu Brain Research Center, Bilkent University, Ankara, Turkey; National Nanotechnology Research Center (UNAM), Bilkent University, Ankara, Turkey; Molecular Biology and Genetics Zebrafish Facility, Bilkent University, Ankara, Turkey; National Magnetic Resonance Research Center (UMRAM), Bilkent University, Ankara, Turkey; Department of Psychology, Bilkent University, Ankara, Turkey

Objective: Environmental enrichment (EE) is one intervention that can lead to the maintenance of cognitive processing in both older humans and animals. EE refers to an exposure to a perceptually, socially and cognitively stimulating environment and is applicable to different model organisms including zebrafish, which show cognitive and cellular decline in a manner similar to humans. The main aim of the current study was to define the molecular underpinnings of EE that promote and retain successful cognitive aging, and focus on factors such as gender and environment, which may alter brain resilience to the aging process.

Methods: Forty-eight young (6-mo.) and forty-five old (27mo.) wildtype zebrafish, including males and females, were randomly assigned to enriched or barren environments. A standard glass $15 \mathrm{~L}$-aquarium was divided into two compartments, an enriched and barren side. Enrichment was achieved via introduction of bottom-gravel, swimming tubes and artificial plants. All groups were maintained in this setup for 5-weeks. Following this intervention, animals were euthanized and whole brains were dissected. Proteins were isolated and subjected to Western blot analysis. Post-synaptic density-95 (PSD95), synaptophysin (SYP), gamma-Aminobutyric-acid-alpha-1 (GABRA1), gluta- mate receptor subunit-2+3 (GLUR2/3) and proliferating-cellnuclear antigen (PCNA) were analyzed.

Results: In SYP levels an aging-related decrease was observed in the barren environment groups, $[\mathrm{F}(1.16)=4.997, \mathrm{p}=.040]$ but animals in the enriched environment tend to maintain the levels of SYP at the older ages, $[\mathrm{F}(1.16)=6.760, \mathrm{p}=.019]$. Excitatory-neurotransmission markers of PSD95 and GLUR2/3 were not altered significantly, but GLUR2/3 levels were increased in enriched-female-young group, $[\mathrm{F}(1,16)=$ 6.457, $\mathrm{p}=.022]$. The proliferation marker PCNA appeared to increase in the enriched group, $[\mathrm{F}(1,16)=3.612, \mathrm{p}=.076]$.

Conclusion: Our data suggest that manipulations of environmental setup might alter the course of synaptic proteins changes during normal aging. Additional studies on the roles of other synaptic and cell-cycle-regulator markers are currently under investigation.

This work was supported by an EMBO Installation Grant.

Keywords: environmental enrichment, aging, synaptic proteins, zebrafish, gender

\section{SS1-A02}

\section{Determination of M1 polarized microglia-derived exosomal micrornas that have an effect on neural stem cell differentiation}

İlkcan Ercan $^{1}$, Çağla Kiser ${ }^{1}$, Uğur Kemal Tüfekci ${ }^{3}$, Melis Olcum², Defne Engür ${ }^{1}$, Abdullah Kumral' ${ }^{2}$, Şermin Genç ${ }^{2}$

'Department of Genomics and Molecular Biotechnology; Izmir Biomedicine and Genome Institute, Dokuz Eylül University, Izmir, Turkey; 'Izmir Biomedicine and Genome Center, Izmir, Turkey; ${ }^{3}$ Vocational School of Health Services, Izmir Democracy University, Izmir, Turkey

Objective: Microglia, the resident cells of the CNS, play roles in different phases of developmental stages. Microglial cells external signals and polarizes into differential polarization phenotypes depending on their recognizing characteristic sense. M1 phenotype microglia demonstrate mainly phagocytic characteristic, whereas anti-inflammatory response is observed in M2 phenotype. Aforementioned cells are known to support neurogenesis under physiological conditions. Exosomes are the vesicular structures secreted from all cells including microglia. The miRNAs secreted from exosomes are taken by the neighboring cells and influence the functions of the recipient cells through their cargo molecules such as protein, mRNA and microRNA (miRNA). The aim of the study is to investigate the effects of M1 polarized microglia on neural differentiation and the differential exosomal miRNA mediating the process.

Methods: Neural stem cells differentiated from CGR8 mouse embryonic stem cells and LPS induced M1 polarized N9 
mouse microglial cells within 3D culture have been used to mimic microenvironment within the brain tissue. The effects of M1 polarized microglia on neural stem cells have been investigated via quantitative PCR method. Exosomes were isolated from M1 polarized N9 microglia and the differential miRNA content of these exosomes were determined via qPCR panel.

Results: Along with M1 polarization, increase in MAP2 mature neural marker, decrease in immature neural markers Doublecortin and BetaIII Tubulin mRNA levels, decrease in oligodendrocyte markers Olig1 and Olig2 have been identified. Among mouse immune pathogenic miRNAs, upregulation of 14 miRNAs and downregulation 26 miRNAs have been determined.

Conclusion: Thereby, it has been determined that these stem cells, due to the effects of M1 polarized microglia, tend to neural differentiation. It is suggested that the effect of polarized microglia on neural differentiation is mediated by the exosomal miRNAs.

This study is supported by TUBITAK. (Project No: 118S406; 218S527)

Keywords: exosome, microglia, miRNA, neural stem cell, polarization

\section{SS1-A03}

\section{Chemoarchitecture of the human brainstem}

Gülgün Șengül ${ }^{1}$, Uğur Türe², George Paxinos ${ }^{3}$

'Department of Anatomy, School of Medicine, Ege University, Izmir, Turkey; ${ }^{2}$ Department of Neurosurgery, School of Medicine, Yeditepe University, Istanbul, Turkey; ${ }^{3}$ Neuroscience Research Australia, Randwick, Sydney, Australia

Objective: Brainstem is of great significance being responsible in vital functions such as respiration, locomotion, arousal as well as cognition and affection. Nuclei in brainstem have been related to diseases such as progressive supranuclear palsy, multiple system atrophy, autism spectrum disorders and Parkinson's disease. In this study, the neurochemical content of the human brainstem was investigated using histochemical and immunohistochemical stainings.

Methods: Brainstem sections were cut using a cryostat at a thickness of 60 micrometers and stained using histochemical (Nissl and acetyl cholinesterase) and 18 different immunohistochemical markers (GABA, glutamate, substance P, NeuN, calbindin, calretinin, parvalbumin, calcitonin-gene-related peptide, enkephalin, galanin, glycine, glial fibrillary acidic protein, cocaine-amphetamine-related peptide, neuropeptide Y, choline acetyl transferase, tyrosine hydroxylase, neuronal nitric oxide synthase, vasoactive intestinal peptide, anti-neurofilament antibody) and brainstem nuclear organization and chemoarchitecture were analyzed.

Results: The localizations of GABA, glutamate, substance P, NeuN, calbindin, calretinin, parvalbumin, calcitonin-generelated peptide, enkephalin, galanin, glycine, glial fibrillary acidic protein, cocaine-amphetamine-related peptide, neuropeptide $\mathrm{Y}$, choline acetyl transferase, tyrosine hydroxylase, neuronal nitric oxide synthase, and vasoactive intesinal peptide were identified in the human brainstem.

Conclusion: With the findings of this study, the chemoarchitecture of the human brainstem was presented in detail and systematically for the first time in the literature.

Keywords: atlas, brainstem, chemoarchitecture, cytoarchitecture, human

\section{SS1-A04}

The effect of $\mathrm{N}, \mathrm{N}$-dimethyltryptamine on the fractal dimension of neurite branching

Muzaffer Beyza Ozansoy ${ }^{1}$, Mehmet Ozansoy ${ }^{2}$

'Department of Physiology, School of Medicine, Istanbul Aydin University, Istanbul, Turkey; 'Department of Physiology, School of Medicine, Bahçeşehir University, Istanbul, Turkey

Objective: N,N-Dimethyltryptamine (DMT) is a hallucinogen synthesized in the peripheral tissues of the body. Although the psychoactive effects of DMT have been well-known, its physiological function in the central nervous system remains elusive. DMT is an endogenous ligand of Sigma-1R (Sig-1R) receptor, playing important roles in neurite outgrowth and cell survival. Neurite outgrowth is one of the basic processes of neuronal network formation, plasticity and development of nervous system. The fractal dimension of neurite branching provides invaluable information about the development and the degree of informational complexity of neuronal networks. The aim of this study is to investigate the possible roles of DMT and cutamesine (SA4503), a selective agonist of Sig-1R, on the fractal dimensions of neurite branches.

Methods: Primary hippocampal neurons isolated from neonatal BalbC mice $(n=20)$ were cultured. The presence of Sig-1R was proved by western blotting and immunocytochemistry. Three experimental groups were designed: Control, DMT (10 $\mu M)$ and SA4503 $(10 \mu M)$. Neurites were visualized by using beta III tubulin primary antibody in laser confocal microscopy. In total, 300 neurons having neurites were selected, and fractal dimensions of neurite branchings were calculated by using the FracLac plugin of ImageJ software. Data were statistically assessed by using one-way ANOVA test.

Results: The fractal dimension of neurite branchings in DMT group is significantly higher when compared with control $(\mathrm{p}<0.01)$. It was also greater than that of SA4503 group $(\mathrm{p}<0.05)$. On the other hand, there was no statistically significant difference in the fractality between the SA4503 group and the control group.

Conclusion: With this study the effects of DMT and the selective agonist of Sig-1R on the fractal dimensions of neurite branchings were investigated for the first time. Our findings indicate the involvement of DMT in the informational complexity of neuronal networks by increasing the fractality of neurite branchings.

Keywords: sigma-1 receptor, neurite branching, fractal dimension, DMT, hippocampal neuron 


\section{SS1-A05}

Age-related morphological and physiological changes of granule cells in the dentate gyrus region responsible for neurogenesis in Wistar rats

Illknur Dursun $^{1}$, Birsen Elibol ${ }^{2}$

'Department of Physiology, Faculty of Medicine, Istinye University, Istanbul, Turkey; ${ }^{2}$ Department of Medical Biology, Faculty of Medicine, Bezmialem Foundation University, Istanbul, Turkey

Objective: Adult hippocampal neurogenesis is important for prevention/treatment of neurodegenerative diseases and impairments in learning/memory, cognition and emotional functions occuring with age. The morphological development of granule cells in Dentate Gyrus (DG) of adult brain provides important clues to researchers. Herein, granular cell morphology alterations in rat hippocampus with age were examined in detail.

Methods: The morphological features of granule cells in DG region of hippocampus at $\mathrm{P} 1, \mathrm{P} 10, \mathrm{P} 30$ and $\mathrm{P} 60$ ( $\mathrm{P}=$ postnatal day) were examined with Neurolucida programme by Golgi staining. Also, changes in protein amount of Doublecortin as neurogenesis marker, synaptophysin for presynaptic neurons, and PSD95 for postsynaptic neurons were determined by immunofluorescence.

Results: A significant increase was observed in granule cells' soma size and dendrite number between P1-P10, dendritic area/dendrite length between P1-P30, dendrite branch number between P10-P30. Dendritic branche angle was the highest at P1. Tortuosity in dendrite branches varied and dendrite number decreased between P10-P30, in addition, branch thickness of dendrites decreased between P1-P10. Dendritic spine density increased up to P30 and decreased slightly at P60. The stubby type dendritic spine were increased at $\mathrm{P} 1$, then, decreased with age. The axon length and axonal area increased significantly at P10 and decreased in later ages. Tortuosity of axon was mostly seen at P10. While the amount of DCX was the highest at P1, this amount decreased over time and increased again at P60. The amount of SYP increased at P30 and the amount of PSD95 increased at P10

Conclusion: The most distinctive morphological changes in granule cells occur especially in brain growth spurt period. The synaptic changes occurs especially between P10-P30. This study contributes to understanding of the mechanisms between morphology and function by examining the hippocampal granule cells, which forms the regeneration of the adult brain for evaluation of treatment alternatives against neurodegeneration.

Keywords: neurogenesis, hippocampus, dentate gyrus, granule cells, rat

\section{SS1-A06}

Effects of perinatal stress on neuroligin-1 and neuroxin Il $\alpha$ immunreactivity in different structural parts of the rat enteric nervous system

Ece Alim $^{1}$, Özen Akarca Dizakar², İsmail Nadir Gülekon², Emel Ulupınar ${ }^{3}$, Ece Konaç ${ }^{4}$, Meltem Bahçelioğlu ${ }^{5}$

'Department of Anatomy, School of Medicine, Gazi University, Ankara, Turkey; Neuroscience and Neurotechnology Center of Excellence NÖROM,
Ankara Turkey; '2Department of Anatomy, School of Medicine, Gazi University, Ankara, Turkey; 'Department of Anatomy, School of Medicine, Eskişehir Osmangazi University, Eskişehir, Turkey; Neuroscience and Neurotechnology Center of Excellence NÖROM, Ankara Turkey; ${ }^{4}$ Department of Medical Biology and Genetics, School of Medicine, Gazi University, Ankara, Turkey; 'Department of Anatomy, School of Medicine, Gazi University, Ankara, Turkey, Neuroscience and Neurotechnology Center of Excellence NÖROM, Ankara Turkey, Neuroscience Department, Institute of Health Sciences, Gazi University, Ankara Turkey

Objective: The relationship between enteric nervous system, gastrointestinal function and stress is very important in clinical and pathophysiological aspects. Neuroligin and neuroxins are synaptic cell adhesion molecules that play a key role in synaptic functions. In recent studies, it is thought that neurologin and neuroxins play an important role in the detection of various cognitive diseases. The bidirectional communication between the brain and the gut, including neuronal, immune and endocrine pathways, is called the brain-gut axis. This axis is affected by various stress factors. In our study, we aimed to investigate the effects of perinatal stress on neuroligin- 1 and neuroxin II $\alpha$ in the enteric nervous system of different intestinal parts of rats at different embryonic / neonatal days.

Methods: In our study, 36 female Wistar albino rats were mated. The experimental animals born with the embryos taken at embryonic days E16, E18, E20. of pregnant rats and raised with their mothers until they were weaned (up to postnatal 21st day) were used. There are 8 groups in total in the experiment. Prenatal and/or postnatal stress protocols were applied to the experimental groups. Immunepressions of neuroligin-1 and neuroxin II $\alpha$ in myenteric plexuses were evaluated of duodenum, jejunum, ileum and colon tissues.

Results: When evaluated statistically, the decrease in the percentages of immunoreactivity density and cell positive numbers of neuroligin-1 and neuroxin II. in the myenteric plexuses in the stress groups compared to the control groups is remarkable $(\mathrm{p}<0.05)$.

Conclusion: Perinatal stress affected the neuroligin-1 and neuroxin II $\alpha$ immunoreactivities in the enteric nervous system.

The study was supported by Tübitak SBAG 3001 (Project no 118S031).

Keywords: enteric nervous system, perinatal stress, neuroligin1, neuroxin II $\alpha$

\section{SS1-A07}

The role of microglial cathepsin Z in Alzheimer's pathology: a study on post-mortem cortex tissue samples taken from Alzheimer's patients

\section{Gürol Atar ${ }^{1}$, Bente Finsen ${ }^{2}$}

'Interdisciplinary Neurosciences, Eskişehir Osmangazi University, Eskişehir, Turkey; '2Department of Molecular Medicine, University of Southern Denmark, Odense, Denmark

Objective: Alzheimer's disease is characterized by various neuropathological findings such as atrophy in the brain, accumula- 
tion of incorrectly folded proteins, neurofibrillary tangles, loss of neuron and synapse. Cathepsins are proteases that are expressed by all brain cell types, and most of them are found both intracellularly and extracellularly. Microglia expresses and secretes several different cathepsins that have supportive roles on various immune functions of it. Cathepsin Z (CtsZ), a lysosomal cysteine protease, is highly expressed in cells of the immune system. The study aims to characterize the expression levels of microglial Cts $Z$ and to examine its distribution on post-mortem cortex tissues obtained from Alzheimer's patients and non- Alzheimer's disease.

Methods: The research was carried out using 8 fresh-frozen post-mortems neocortical tissue from $\mathrm{AD}$ and non-demented cases were provided by the Netherlands Brain Bank. Doublestaining immunohistochemistry technique was used (Iba1 microglia-CtsZ cathepsin Z).The digitization and counting of the cells were done using the Fiji program. IBM SPSS 25 program was used for analysis.

Results: We found that the levels of CtsZ expression $(M=70.90 \mathrm{SE}=11.20)$ in the post-mortem cortex tissues of Alzheimer's patients were higher than those of the people nonAlzheimer's disease $(M=35.62 \mathrm{SE}=16.06)$. Mann Whitney U was used to test whether this difference was significant. There was no significant difference between the two groups in terms of the level of CtsZ expression ( $\mathrm{p}=0.09>0.05)$.

Conclusion: We found that there was an increase in the expression level of CtsZ in the post-mortem cortex tissues of Alzheimer's patients. Adversely activated microglia may have contributed to inflammation-induced neurodegeneration by secreting CtsZ. Considering that Cathepsin $\mathrm{Z}$ is expressed in the lysosomes of brain macrophage cells located near the AB and Tau aggregates in the neuropil, It is thought that CtsZ interacts with them. Detailed research is needed to elucidate the interactions of CtsZ with microglial cells in Alzheimer's pathophysiology.

Keywords: Alzheimer's disease, cathepsin Z, cysteine proteases, microglia

\section{SS1-B}

\section{Behavioral and Cognitive Neuroscience}

\section{SS1-B01}

The role of dorsolateral prefrontal cortex in figurative language processing: an rTMS study

Hazel Zeynep Kurada ${ }^{1}$, Elif Arıca Akkök², Zerin Özaydın Aksun ${ }^{3}$, H. Özden Şener

'Department of Speech Language Therapy, Hacettepe University, Ankara, Turkey; 'Linguistics Department, Ankara University, Ankara, Turkey; ${ }^{3}$ Department of Neurology, Ankara University, Ankara, Turkey

Objective: Current study aims to investigate the cerebral lateralisation phenomena specific to the literal versus figurative language processing.
Methods: To further test the contribution of dorsolateral prefrontal cortex in both right and the left hemisphere during the processing of figurative meaning and literal meaning, we used an interference approach by means of repetitive transcranial magnetic stimulation (rTMS). Eighteen young healthy participants (mean age: 20.72) underwent rTMS pulses at $1 \mathrm{~Hz}$ frequency, $110 \%$ of motor threshold intensity for 15 minutes (900 pulses) in two sessions at one-week interval. The target DLPC positions (right and left) of each participant were determined with Modified Beam Method (Beam et.al., 2009). A semantic judgement task comprised of idioms (for figurative meaning) and literal sentences (for literal meaning) was performed on a PC. Participants have been asked to judge the relatedness of an idiom (or a literal expression) and a following target word by pressing a button on a response key. The target word was either figuratively related or literally related to the idiom. Reaction times and accuracy scores data from semantic judgement task were analysed using linear mixed effects models.

Results: Results revealed that bilateral DLPC plays a role in figurative language processing however left DLPC has been observed more critical during figurative meaning processing than that of right DLPC. Unlike figurative meaning processing, no such hemispheric or stimulation effects were observed for literal meaning processing. Besides the left DLPC functions are critical in the selection but not supression of one of two competing interpretations that are activated in terms of literal and figurative meaning of an idiom.

Conclusion: Left DLPC functions are more critical in figurative meaning processing than the right DLPC. General DLPC functions are not critical for literal meaning processing. The selection and inhibiton components are localised in different cortical areas.

Keywords: DLPC, figuration, idioms, transcranial magnetic stimulation

\section{SS1-B02}

Investigating the role of attentional processes on mental number representations: an fMRI study

Funda Salman, Banu Cangöz

Department of Psychology, Hacettepe University, Ankara, Turkey

Objective: According to Triple Code Model (TCM) proposing serial processing (SP), numbers are encoded and activated in three different independent representations (analogic-magnitude, visual-Arabic-number, auditory-verbal), and transcoded into each other via SP. According to Encoding Complex Model (ECM) proposing parallel processing $(\mathrm{PP})$, numbers are encoded in complex codes and activated simultaneously through associations of these codes in different strengths. Contemporary views concerning attention and memory in cognitive psychology argue PP is theoretically more effective in mental mechanisms. This research aims to investigate the role of PP via $\mathrm{AMRI}$ images while explaining mental number representations in different ages based on attentional networks. 
Methods: Twenty healthy volunteers consisting of children and adults without any diagnosis, who obtained appropriate scores from the neuropsychological and psychiatric tests/scales due to norms, participated to the study. Behavioral and fMRI records were obtained from participants while performing executive attention component of Nonorthogonal-Attentional Network Test, which was recreated as implementing analogical and Arabic-number stimuli into the task.

Results: According to mixed ANOVA results (reaction time), main effects and interactions of age (children>adults) and executive attention (distractor present>absent) are significant. The main effect of representational codes (analogical>Arabic-number) is significant. SNARC effect (incongruent>congruent) is obtained when the distractor is absent in both of the representations in children, whereas it is not obtained in adults. According to fMRI results (BOLD amplitude) ( $<<0.05$, FWE correction), left/right precuneus, right inferior/medial/superior frontal gyrus regions in analogical representation, and left parahippocampal gyrus in Arabicnumber representation are active. The left superior temporal gyrus is activated during Arabic-number representation in children.

Conclusion: Obtaining the brain regions of all representational codes including analogical, Arabic-number and verbal is consistent with the literature and supports ECM which argues simultaneous activations in all representations. When behavioral and fMRI results are evaluated together, TCM and ECM may differ in accounting for number processing due to age.

Keywords: number processing models, analogical representation, Arabic number representation, executive attention, fMRI

\section{SS1-B04}

\section{Biological motion perception in perceptual decision making framework}

Berfin Aydın, Burcu Ayşen Ürgen

Interdisciplinary Neuroscience Program \& Aysel Sabuncu Brain Research Center, Bilkent University, Ankara, Turkey

Objective: Neurophysiological studies in non-human primates show that single neurons in LIP mediate perceptual decision making. These studies which usually employ a random dot motion paradigm show that as the coherence of the stimulus is increased, response time and miss rate decreases, and firing rate increases. EEG studies in humans which use similar paradigms show consistent results with literature. It remains unknown whether these processes generalize to more complex stimuli. The aim of the present study is to investigate decision making processes that underlie biological motion perception.

Methods: 9 human subjects participated in a behavioral experiment. Every trial started with a 2 -second fixation point, followed by a scrambled motion stimulus with noise dots at different difficulty levels $(10,20,30,40,50)$ that were added to manipulate the coherence of the stimuli. This was followed by a biological motion stimulus (a human walker that walks towards left or right) which have the same number of noise dots. The task was to decide the direction of the walker within
2 seconds. If there was no response in 2 seconds, the trial was marked as a missed trial.

Results: Our results show that response time increased as task difficulty was increased (or the coherence was decreased). A one-way ANOVA on the response times showed the main effect of the difficulty level $[\mathrm{F}(1.98,15.87)=54.791, \mathrm{p}<0.001]$. In addition, we also found that the miss rate increased as task difficulty was increased. A one-way ANOVA on the miss rate showed the main effect of the difficulty level $[\mathrm{F}(1.55,12.39)$ $=27.243$, $\mathrm{p}<0.001]$.

Conclusion: The present study suggests that decision making during biological motion perception becomes more difficult as the coherence of the stimuli is decreased. These results extend previous work on motion perception and suggests that similar decision making mechanisms may play role in biological motion perception.

Keywords: biological motion, decision making, visual perception

\section{SS1-B05}

\section{Gender differences modulate visual event-related oscillations}

Duygu Hünerli Gündüz ${ }^{1}$, Seren Düzenli Öztürk², Deniz Yerlikaya ${ }^{1}$, Görsev Yener ${ }^{3}$

'Department of Neurosciences, Institute of Health Sciences, Dokuz Eylül University, Izmir, Turkey; ${ }^{2}$ Department of Speech and Language Therapy, Izmir Bakırçay University, Izmir, Turkey; ${ }^{3} / z m i r$ Biomedicine and Genome Center, Dokuz Eylül University Health Campus, Izmir, Turkey

Objective: In the present study, we aimed to investigate eventrelated oscillations (ERO) of female and male healthy volunteers using a classical visual oddball paradigm.

Methods: Ten healthy female and 11 demographically comparable male volunteers between the ages of 21-55 were included in the study. All participants had intact cognitive functions according to neuropsychological test scores. EEG was recorded from F3, Fz, F4, C3, Cz, C4, P3, Pz, P4, O1, Oz, and O2 electrode locations according to international 10-20 system. Maximum peak-to-peak amplitude values of delta $(0.5-3.5 \mathrm{~Hz})$ and theta (4-7 Hz) EROs were automatically measured. Repeated measures ANOVA included Gender (2 levels: female, male) as a betweensubject factor, and Anterior Posterior (AP) Electrode Location [4 levels: frontal, central, parietal, occipital] and Laterality (LAT) [3 levels: left, mid, right] as a within-subject factor.

Results: Repeated measures of ANOVA indicated a main gender effect on peak-to-peak amplitude values of delta $[\mathrm{F}(1.19)=4.694$; $\mathrm{p}=043]$, and theta EROs $[\mathrm{F}(1.19)=14.200 ; \mathrm{p}=001]$. Females had significantly higher delta and theta ERO responses than males. Moreover, there was an interaction effect of Gender $\times \mathrm{AP} \times \mathrm{LAT}$ on delta EROs $[\mathrm{F}(6.114)=3.542 ; \mathrm{p}=.009]$. Post-hoc analyses showed that compared to males, females had significantly higher delta ERO responses at Fz, F4, O1, Oz, and O2 electrode locations (for all; $\mathrm{p}<.039$ ). 
Conclusion: Event-related potentials are revealed with alterations of brain electrical activity with an application of a task, mostly with a cognitive load. P300 is known to be significantly modulated by gender, with higher amplitude in females compared to males. In line with findings of P300, we showed that delta and theta responses, which are the main components of P300, were also higher in females in comparison to males. Gender differences in EEG-ERO is one of the factors that should not be ignored.

Keywords: EEG, event-related oscillations, delta, theta, gender

\section{SS1-B06}

\section{Investigating word-object association in primary progressive aphasia using eye movement tracking and MRI}

İlayda Demir ${ }^{1}$, Ulaş Ay ${ }^{2}$, Kardelen Eryürek ${ }^{1}$, Elif Kurt', Çidem Ulaşoğlu Yıldız', Gülden Güvenç3, İbrahim Hakan Gürvit $^{4}$, Mustafa Seçkin ${ }^{5}$, Tamer Demiralp ${ }^{6}$

'Department of Neuroscience, Aziz Sancar Experimental Medicine Research Institute, Istanbul University, Istanbul, Turkey; 'Neuroimaging Unit, Hulusi Behçet Life Sciences Research Laboratory, Istanbul University, Istanbul, Turkey; ${ }^{3}$ Department of Psychology, Faculty of Arts and Sciences, Işık University, Istanbul, Turkey; ${ }^{4}$ Behavioral Neurology and Movement Disorders Unit, Istanbul Faculty of Medicine, Istanbul University, Istanbul, Turkey; ${ }^{5}$ Department of Neurology, Faculty of Medicine, Acibadem Mehmet Ali Aydınlar University, Turkey; ${ }^{6}$ Department of Physiology, Istanbul Faculty of Medicine, Istanbul University, Istanbul, Turkey

Objective: Primary progressive aphasia (PPA) is a neurodegenerative disease in which language functions are prominently impaired and other cognitive abilities are relatively preserved. A subset of PPA patients (semantic PPA) present with word comprehension impairment associated with disruption of word-object associations while others present with nonsemantic impairment such as word-finding difficulties (logopenic PPA), nonfluent speech and/or agrammatism (agrammatic PPA). In this study, we aim to delineate word-object associations and to identify related areas of atrophy in patients with nonsemantic PPA.

Methods: Eleven PPA patients with relatively preserved word comprehension and 10 healthy controls were included. Participants were given a word-object matching task consisted of 24 trials and on each trial 16 object pictures (one target and 15 distractors) were presented on an elliptical array on a touchscreen. Eye movements were recorded during the task using a remote eyetracker. Cortical thickness maps were created using FreeSurfer software by processing the structural images acquired using a $3 \mathrm{~T}$ scanner.

Results: Both PPA (M=\%91) and control (M=\%99.5) groups completed the word-to-object matching task successfully. PPA group fixated significantly greater number of distractors $(\mathrm{p}<0.01)$, repeatedly $(\mathrm{p}=0.008)$ with longer duration $(\mathrm{p}<0.001)$ compared to the controls. Cortical thickness analysis revealed that PPA group had significant atrophy at the anterior temporal lobe (ATL) as well as at the inferior frontal gyrus and the temporoparietal junction (TPJ) on the left hemisphere.

Conclusion: Our findings reveal that subclinic deterioration in word-object association can be detected at an early stage of PPA by eye movement tracking. Cortical thickness analysis showed atrophy in the left IFG and TPJ regions that are specifically involved in agrammatic and logopenic PPA, respectively. Additional atrophy was found at the left ATL that is specific to semantic PPA suggesting that these patients suffer from a neurodegenerative condition that may evolve to mixed PPA over time.

Keywords: primary progressive aphasia, neurodegeneration, eye tracking, cortical thickness, word-object association

\section{SS1-B07}

The validity and reliability study of the language battery of CAT-TR

\section{Sevket Özdemir ${ }^{1}$, İlknur Maviş}

'Department of Special Education, Hacettepe University, Ankara, Turkey, ${ }^{2}$ Department of Speech and Language Therapy, Anadolu University, Eskişehir, Turkey

Objective: As aphasia assessment is the initial step of a wellstructured language therapy, the assessment tests need to consider the typological and cultural characteristics of the language (Maviş, 2010). A group of international researchers in the Collaboration of Aphasia Trialists (supported by ISCH COST Action IS1208) is adapting Comprehensive Aphasia Test (CAT; Swinburn, Porter, and Howard, 2004) into 14 languages spoken in Europe including Turkish. The aim of this study was to carry out the reliability and validity analyses of the Language Battery section of CAT-TR to ensure the assessment of Turkish-speaking people with aphasia $(\mathrm{PwA})$.

Methods: The test included 21 sub-tests and yielded six modality scores. $90 \mathrm{PwA}$ (mean age: 61.07) and 200 healthy (mean age: 54.89) adults involved in the analyses. The participants were stratified into two education and three age groups.

Results: The analyses belonging to content, construct and criterion validity were carried out, while the reliability analyses were performed on the internal consistency, stability and equivalence. Education was found to influence all the modality scores of the healthy participants, while the age-related differences were significant among all the modality scores except reading. Moreover, female healthy participants demonstrated a higher performance only in the naming modality scores. The education and gender variables did not hold any significant effects on the language performance of PwA, whereas those who were younger than 60 showed statistically better performance in the spoken and written language comprehension modality scores. The cut-off values for the modality and total language scores were provided. 
Conclusion: Compared to the psychometric values of aphasia tests utilized in Turkey and the world, CAT-TR is an appropriate tool for the language assessment of Turkish-speaking adults. Based on the cognitive neuropsychological approach, CAT-TR will assess the word comprehension and production difficulties on a psycholinguistic basis and provide a different perspective to the clinicians.

Keywords: aphasia, assessment, comprehensive aphasia test, reliability, validity

SS1-C

Neuroimaging and Neuromicroscopy

\section{SS1-C01}

Volumetric changes within hippocampal formation subfields in Alzheimer's disease continuum

Emre Harı ${ }^{1}$, Gözde Kızılateş Evin ${ }^{1}$, Ali Bayram², Elif Kurt ${ }^{2}$, Çiğgdem Ulaşoğlu Yıldız ${ }^{1}$, Burak Acar ${ }^{3}$, Tamer Demiralp", İ. Hakan Gürvit

'Neuroimaging Unit, Hulusi Behçet Life Sciences Research Laboratory, Istanbul University, Istanbul, Turkey; ${ }^{2}$ Department of Neuroscience, Aziz Sancar Institute of Experimental Medicine, Istanbul University, Istanbul, Turkey; ${ }^{3}$ Department of Electrical-Electronics Engineering, Faculty of Engineering, Bogaziçi University, Istanbul, Turkey; ${ }^{4}$ Department of Physiology, Istanbul Faculty of Medicine, Istanbul University, Istanbul, Turkey; ${ }^{5}$ Department of Neurology, Behavioral Neurology and Movement Disorders Unit, Istanbul Faculty of Medicine, Istanbul University, Istanbul, Turkey

Objective: Progressive degeneration in Alzheimer's disease continuum (ADC) starts from transentorhinal cortex and progresses within hippocampal formation (HF) structures. The investigation of the neurodegeneration spread pattern through the HF subfields in this continuum is critical for the early detection of $\mathrm{AD}$. In this study, we aimed to track the volumetric changes of the HF subfields along the ADC by using magnetic resonance imaging (MRI).

Methods: Structural MRI data of 15 patients diagnosed with Alzheimer's disease dementia (ADD), 15 amnestic mild cognitive impairment (MCI) and 15 subjective cognitive impairment (SCI) were used, and they were matched in terms of age, education and gender. HF is individually subdivided into dentate gyrus (DG), subiculum (SUB), CA1 and CA3, and volume values were obtained using FreeSurfer software (http://surfer. nmr.mgh.harvard.edu/). ANCOVA analysis with intracranial volume as covariate was performed to evaluate the differences between the groups. Bonferroni correction with $\mathrm{p}<0.005$ was applied on F-test results and with $\mathrm{p}<0.0017$ for $\mathrm{t}$-tests between pairs of groups.

Results: As a result, statistically significant volume reductions were detected among the three groups from SCI towards ADD in bilateral CA1, DG and SUB. In ADD, DG, SUB and CA1 volumes were less compared to SCI. Additionally, the volumes of DG in left hemisphere, SUB and DG in right hemisphere were statistically lower in ADD compared to MCI.

Conclusion: In the volumetric studies conducted with the $\mathrm{ADC}$, volume loss was reported in the early stages, especially in CA1 and SUB, and with a lesser degree in DG. While the results of our study are generally consistent with the literature, they additionally point to importance of the progressive volume loss in DG. This pattern of volume decrease is compatible with tau transsynaptic spread in the intrinsic hippocampal circuit.

Present work was supported by TÜBİTAK ARDEB (Project Nr:114E053) and IU-BAP (Project Nr: 1567/42362).

Keywords: Alzheimer's disease, hippocampal formation, magnetic resonance imaging, volumetry

\section{SS1-C02}

Investigating the effect of aggression on cognitive control by event-related potentials and oscillations

Mustafa Yavuz ${ }^{1}$, Ejder Akgün Yıldırım² ${ }^{2}$ Elif Sarışık, Ertan Yurdakoş ${ }^{4}$, Tamer Demiralp ${ }^{5}$

${ }^{1}$ Neuroimaging Unit, Hulusi Behçet Life Sciences Center, Istanbul University, Istanbul, Turkey; 'Bakırköy Mental Health and Neurological Diseases Education and Research Hospital, Department of Psychiatry, Istanbul, Turkey; ${ }^{3}$ stanbul University, Istanbul Faculty of Medicine, Istanbul, Turkey; ${ }^{4}$ Department of Medical Physiology, Faculty of Medicine, Altınbaş University, Istanbul, Turkey; ${ }^{5}$ Department of Physiology, Istanbul Faculty of Medicine, Istanbul University, Istanbul, Turkey

Objective: Aggression has been associated with reduced cognitive control, impulsivity and declined response inhibition. Such changes in executive control are known to relate with changes in event-related brain potential (ERP) components such as N1 or P3. In present study, electrophysiological reflections of differences among healthy subjects that can be determined by aggression scales were investigated by ERPs and event-related oscillations (ERO).

Methods: Sample consists of 22 university students, 12 with low and 10 high aggression scores. EEG is recorded during classical visual Go/NoGo paradigm. Epochs between -500 to $1000 \mathrm{~ms}$ for each of Go-NoGo conditions were averaged in time to obtain ERPs. Complex wavelet transform applied on ERPs within 3-48 Hz frequency band revealed evoked activity phase-locked to events and average of wavelet powers of single trials of each subject and condition revealed total activity that includes non-phase-locked signal components. ERPs, and evoked and total EROs were compared between two groups by means of cluster-based permutation tests as implemented in Fieldtrip toolbox (http://www.fieldtriptoolbox.org/). ERP and ERO clusters of $\mathrm{p}<0.05$ significance level were reported.

Results: ERP analyses showed that high aggression group displayed a lower positivity between 200-300ms in ERPs to Go events compared with those of low aggression group, while that 
showed significantly higher negativity between $100-160 \mathrm{~ms}$ in NoGo trials. Time-frequency analysis displayed a higher power in total low-beta power $(14-20 \mathrm{~Hz})$ between 120-230 ms in NoGo responses of high aggression group, but a lower beta activity between $720-770 \mathrm{~ms}$ in Go responses.

Conclusion: Our results show that high aggression group shows a higher activation in both time and time-frequency domains in condition when response inhibition is necessary, while resources they can activate in response to target events are limited compared with low-aggression group. Findings provide new information about neurophysiological basis of relationship of aggression with cognitive control processes.

Keywords: aggression, cognitive control, cluster-based permutation tests, event-related potentials, event-related oscillations

\section{SS1-C03}

Toward understanding neural mechanisms of active sensing in weakly electric fish

Ísmail Uyanık $^{1}$, Noah J Cowan ${ }^{2}$, Eric S Fortune ${ }^{3}$

'Department of Electrical and Electronics Engineering, Hacettepe University, Ankara, Turkey; 'Department of Mechanical Engineering, Johns Hopkins University, Baltimore, MD, USA; ${ }^{3}$ Department of Biological Sciences, New Jersey Institute of Technology, Newark, NJ, USA

Objective: Sensory and motor systems are linked: motor actions alter the information that animals receive from their sensory receptors, which in turn is used to control subsequent motor actions. In active sensing, animals execute ancillary motor movements to obtain and/or modulate sensory information. The neural mechanisms for the control of active sensing are not known. Our goal is to describe the neurophysiological interactions between the sensory and motor circuits in the CNS toward understanding the neural mechanisms of the active sensing.

Methods: Weakly electric fishes such as Apteronotus leptorhynchus robustly perform an image stabilization task in which animals track the movement of a refuge in a single linear dimension. During this behavior, these animals produce large fore-aft movements for active sensing that modulate and maintain a robust level of sensory slip, the main form of feedback used in the control of refuge tracking. We performed chronic tetrode recordings in midbrain circuits of $(n=17)$ Apteronotus during their free refuge tracking behavior to reveal the sensorimotor activity related to the control of these active sensing movements. We implanted electrodes in the Torus semicircularis, a midbrain electrosensory area where direction-selective responses first emerge.

Results: We found direction-selective neurons, which respond to a range of velocities for the sensory slip, the error between the refuge and fish movements. Our analysis revealed that these direction-selective neurons are also selective for ranges of acceleration of the sensory slip. Midbrain electrosensory neu- rons responded best to high-frequency features, behaving as a high-pass filter on the sensory slip.

Conclusion: The behavioral studies suggest high-pass sensorimotor transformations in the CNS. The spatiotemporal filtering properties of the midbrain neurons we obtained matched the predictions of the behavioral experiments. These preliminary findings are a first step toward understanding neural mechanisms for control of active sensing in a freely behaving animal.

Keywords: active sensing, neural code, neuroethology, sensorimotor control

\section{SS1-C04}

Relationships between the functional and structural neuroimaging findings in Alzheimer's disease

Mesut Canl ${ }^{1}$, Emre Harı ${ }^{2}$, Gözde Kızılateş Evin ${ }^{2}$, Onurhan Karatay ${ }^{1}$, Elif Kurt ${ }^{3}$, Çiğgdem Ulaşoğlu Yıldız ${ }^{2}$, Ali Bayram ${ }^{3}$, Burak Acar ${ }^{5}$, İ. Hakan Gürvit ${ }^{4}$, Tamer Demiralp ${ }^{1}$ 'Department of Physiology, Istanbul Faculty of Medicine, Istanbul University, Istanbul, Turkey; ${ }^{2}$ Hulusi Behcet Life Sciences Research Laboratory, Neuroscience Unit, Istanbul University, Istanbul, Turkey; ${ }^{3}$ Department of Neuroscience, Aziz Sancar Institute of Experimental Medicine, Istanbul University, Istanbul, Turkey; ${ }^{4}$ Department of Neurology, Istanbul Faculty of Medicine, Behavioral Neurology and Movement Disorders Unit, Istanbul University, Istanbul, Turkey; ${ }^{5}$ Department of Electrical and Electronics Engineering, Faculty of Engineering, Boğaziçi University, Istanbul, Turkey

Objective: Typical Alzheimer's disease (AD) is characterized by the atrophy of medial temporal lobes and hippocampi as core structures, which is accompanied by progressive cognitive decline. This progressive neurodegeneration is observed as volume reductions in the magnetic resonance (MRI) images that start in the core structures and spread to the rest of the brain. Investigation of relationships between volume loss and functional MRI (fMRI) data is important in evaluating the neurobiology of $\mathrm{AD}$. In this study, associations between volume changes of gray matter and the amplitude of low frequency fluctuations (ALFF) of the blood oxygen level dependent (BOLD) signal were investigated.

Methods: The study group consisted of 21 early-stage Alzheimer's Disease Dementia (ADD) patients and 33 healthy controls (HC). The ALFF value of each voxel was obtained by using AFNI (https://afni.nimh.nih.gov/) program after preprocessing with CONN package (https://web.conn-toolbox.org/). Gray matter volumes were calculated using CAT12 software (http://www.neuro.uni-jena.de/cat/). For the comparison of both structural and functional data between the two groups by means of t-tests cluster-level significance threshold was set at $\mathrm{pFWE}<0.05$.

Results: Overlapping regions of significant differences of functional and structural data between the two groups were the parahippocampal gyrus, hippocampus and amygdala in the left 
and the temporal pole, parahippocampal gyrus, and hippocampus in the right hemisphere. In these regions, ALFF values significantly increased while volumes were significantly reduced in ADD compared to HC.

Conclusion: While the literature reports reduced functional connectivity in $\mathrm{fMRI}$ of the neurodegeneration areas in $\mathrm{AD}$, our results show that the amplitudes of the BOLD fluctuations in these areas do not decrease but in contrary significantly increase. These findings suggest that the increased BOLD fluctuations may reflect the compansatory or dysfunctional activities of the neural circuits in neurodegeneration areas.

Present work was supported by TÜBİTAK ARDEB (Project Nr:114E053) and IU-BAP (Project Nr: 1567/42362).

Keywords: Alzheimer's disease, ALFF, voxel-based morphometry, magnetic resonance imaging

\section{SS1-C05}

Cortical thickness, neurocognition and social cognition in euthymic individuals with bipolar disorder and their healthy siblings

Burcu Verim $^{1}$, Burak Erdeniz ${ }^{2}$, Emre Bora ${ }^{3}$, Deniz Ceylan ${ }^{4}$, Ayşegül Ildız ${ }^{1}$, Ayşegül Özerdem ${ }^{5}$

'Department of Neuroscience, Institute of Health Sciences, Dokuz Eylül University, Izmir, Turkey; '2Department of Psychology, Faculty of Arts and Sciences, Izmir University of Economics, Izmir, Turkey; ${ }^{3}$ Department of Neuroscience, Institute of Health Sciences, Dokuz Eylül University, Izmir, Turkey; Department of Psychiatry, Faculty of Medicine, Dokuz Eylül University, Izmir, Turkey; Melbourne Neuropsychiatry Centre, Department of Psychiatry, Faculty of Medicine, University of Melbourne, Vic, Australia; ${ }^{4}$ Department of Psychiatry, Faculty of Medicine, Izmir University of Economics, Izmir, Turkey; ${ }^{5}$ Department of Psychiatry and Psychology, Mayo Clinic, Rochester, MN, USA

Objective: The relationship between the genetic roots of bipolar disorder (BD), cognitive impairments and changes in the brain structure has not been elucidated yet. The aim of this study was to investigate the link between genetic liability to $\mathrm{BD}$, neurocognitive and social cognitive impairments and alterations in cortical thickness.

Methods: 24 euthymic patients with BDI according to DSM IV, 16 healthy siblings and 24 healthy controls completed a test battery measuring neurocognition and social cognition and underwent 3 Tesla T1 weighted MRI scanning session. For preprocessing and analysis of the brain imaging data, SPM software and CAT12 toolbox were used. ANOVA was used for the intergroup comparisons of the cortical thickness measurements, of the scores from the cognitive tests and of the continuous demographic variables, and chi-square test was used for the categorical variables.

Results: Compared to controls, patients scored significantly lower in visual memory $(\mathrm{F}=4.48, \mathrm{p}=0.01)$ and mental state decoding $(\mathrm{F}=4.24, \mathrm{p}=0.01)$ whereas siblings in mental state rea- soning $(\mathrm{F}=5.38, \mathrm{p}=0.007)$. Patients and siblings presented decreased cortical thickness in superior frontal gyrus and precentral gyrus $(\mathrm{F}=12.61, \mathrm{pFWE}<0.001)$ and in inferior temporal and postcentral gyrus $(\mathrm{F}=4.08, \mathrm{pFWE}<0.05)$ respectively. Finally, cortical thickness of the siblings were greater in the rostral middle frontal gyrus and the medial orbitofrontal cortex $(\mathrm{F}=4.08, \mathrm{pFWE}<0.05)$ compared to the patients.

Conclusion: Impairment in certain domains of social cognition together with lower cortical thickness in both BDI patients and siblings may be considered as signs of genetic liability for BD which may develop into markers for early diagnosis. Additionally, increased cortical thickness in the siblings compared to the patients may be a marker of resilience for BD.

Keywords: bipolar disorder-I, healthy siblings, cortical thickness, neurocognition, social cognition

\section{SS1-C06}

Medium and long-term effect of chronic smoking on retinal nerve fiber layer

Mahmut Atum

Department of Ophthalmology, Faculty of Medicine, Sakarya University, Sakarya, Turkey

Objective: The study aims to investigate the medium and long-term effects of smoking on the retinal nerve fiber layer (RNFL).

Methods: The study was conducted with 66 non-smoking patients (Group 1), 76 patients (Group 2) with smoking duration between 10-20 years and 74 patients with smoking duration >20 years (Group 3), who admitted the ophthalmology clinic for various reasons between January 2018 and May 2019. Intraocular pressure (IOP), central corneal thickness (CCT), average and regional (superior, inferior, nasal and temporal quadrants) RNFL thicknesses of all participants were measured with OCT and recorded for comparison among 3 groups. ANOVA test was applied in group comparisons. Bonferroni correction was performed in the comparisons between the groups. The Pearson correlation analysis test was used for the correlation analysis of different variables.

Results: There was no statistically significant difference between the groups in terms of gender, age, IOP and CCT values $(p>0.05)$. There were a statistically significant difference between the groups in the average RNFL and superior quadrant $R$ NFL values ( $\mathrm{p}=0.013, \mathrm{p}=0.032$, respectively, ANOVA). Also, there was no statistically significant differences between the groups in the average RNFL values of the nasal, inferior and temporal quadrants $(\mathrm{p}=0.230, \mathrm{p}=0.153, \mathrm{p}=0.581$, respectively, ANOVA). There was a significant negative correlation between smoking duration and average RNFL, superior RNFL and temporal RNFL(r=-0.325, $\mathrm{p}<0.05, \mathrm{r}=-0.366, \mathrm{p}<0.05, \mathrm{r}=-$ $0.308, \mathrm{p}<0.05$, respectively). 
Conclusion: A significant relationship was found between chronic smoking and average, and superior quadrant RNFL thicknesses.

Keywords: optic coherence tomography, smoking, retina

\section{SS1-C07}

Comparison of surface based and voxel based approaches in cortical thickness calculations

$\underline{\text { Ulaș Ay }}{ }^{1}$, Gözde Kızılateş Evin ${ }^{1}$, Elif Kurt'², Çiğdem Ulaşoğlu Yıldız', Kardelen Eryürek², Ali Bayram², Başar Bilgiç ${ }^{3}$, Tamer Demiralp ${ }^{4}$

'Hulusi Behçet Life Sciences Research Laboratory, Neuroimaging Unit, Istanbul University, Istanbul, Turkey; 'Department of Neuroscience, Aziz Sancar Experimental Medicine Research Institute, Istanbul University, Istanbul, Turkey; ${ }^{3}$ Department of Neurology, Istanbul Faculty of Medicine, Behavioral Neurology and Movement Disorders Unit, Istanbul University, Istanbul, Turkey; ${ }^{4}$ Department of Physiology, Istanbul Faculty of Medicine, Istanbul University, Istanbul, Turkey

Objective: The two main analysis approaches for automatically calculating cortical thickness (CT) from anatomical MR images are based on surface-based or voxel-based techniques. This study aims to compare the CT measurements obtained from these two approaches and determine the consistency of both methods in test-retest (TRT) measurements.

Methods: MR images from 424 subjects without any neurological/psychiatric disease who participated as control subjects in various studies conducted at Istanbul University, Hulusi Behçet Life Sciences Research Laboratory were analyzed. The analyses were carried out by using the surface-based FreeSurfer (FS) and voxel-based CAT12 softwares, and CT values were calculated according to the Desikan-Killiany atlas. TRT analyses were performed on the data of 60 participants. The significance value for the t-tests was set as $\mathrm{p}<0.0007$, with Bonferroni correction for the number of anatomic parcels investigated.

Results: In all regions except the left caudal anterior cingulate cortex, left parahippocampal cortex, and right temporal pole, the CT values measured by CAT12 were higher than those measured by FS (the largest p-value $=0.000002$ ). CTs of the anatomical regions showed a high correlation between the two methods $\left(\mathrm{R}^{2}=0.75\right)$. The TRT variability of the two approaches showed similar results $\left(\mathrm{FS} \mathrm{R}^{2}=0.95\right.$; CAT12 $\left.\mathrm{R}^{2}=0.95\right)$. For both methods, there was no anatomic region that showed significant TRT variability.

Conclusion: In almost all anatomical regions, the CT values calculated by CAT12 were significantly higher than those calculated by FS. However, taking into account the high correlation between CT values obtained by both methods across the anatomical parcels and the high TRT consistency of each method, we conclude that both techniques can provide equally valid results for between subjects or groups comparisons or follow-up studies as long as they are not mixed with each other.

This work was supported by IU-BAP (Project No: 1567/ 42362).

Keywords: cortical thickness, CAT12, FreeSurfer
SS2-A

Neural Networks and Computational Neuroscience

\section{SS2-A02}

On the entropy of brain anatomic regions for complex problem solving

Gönül Günal Değirmendereli ${ }^{1}$, Fatoş T. Yarman Vural ${ }^{2}$, Sharlene D. Newman ${ }^{3}$

'Department of Cognitive Science, Middle East Technical University, Ankara, Turkey; ${ }^{2}$ Department of Computer Engineering, Middle East Technical University, Ankara, Turkey; ${ }^{3}$ Department of Psychological and Brain Sciences, Indiana University, Bloomington, IN, USA

Objective: In this study, we aim to measure the information content of brain regions to examine the active anatomic regions for cognitive states of human subjects based on fMRI data. We also aim to detect brain networks corresponding to those cognitive states.

Methods: We propose an information theoretic method for analyzing the activity in/between anatomic regions. The fMRI data was recorded while the subjects played a computerized version of Tower of London game. The subjects are 18 individuals, between 19-38 years old. We investigate the relationship between the problem-solving task phases and the Shannon entropy measures, for the underlying brain activity during a Tower of London problem solving process. We define and estimate two types of Shannon entropy, static and dynamic entropy, to understand how complex problem solving processes lead to changes in information content of anatomic regions. We also use Kullback-Leibler (relative entropy) measure to quantify the connections among anatomical regions.

Results: We observe that entropy measures successfully identify the active brain regions which participate the complex problem solving task. We show that the low entropy regions have relatively higher entropy measures for the resting states compared to that of the complex problem solving states. It is, also, observed that low entropy regions have relatively higher entropy values for the execution phase when compared to the planning phase of problem solving. Finally, expert players have lower entropy values compared to novice players. We see that static and dynamic entropy measures of anatomic regions are highly consistent with the experimental findings of neuroscience.

Conclusion: The preliminary results show strong promise in using the suggested static and dynamic entropy as a measure for characterizing the brain states related to the problem solving process. This capability would be useful in revealing the hidden cognitive states of subjects performing a specific cognitive task.

Keywords: entropy, fMRI, brain decoding, brain networks, complex problem solving 


\section{SS2-A03}

An evolved model of locomotion: visual control of acceleration and braking

Didem Kadıhasanoğlu

Department of Psychology, TOBB University of Economics and Technology, Ankara, Turkey

Objective: This work aims to investigate the visual information used by humans to control acceleration and deceleration while approaching an object. The underlying theoretical framework is the optic flow based perception developed by James J. Gibson. An evolutionary robotics model of a simple locomotion task is developed to test the effectiveness of five different optical variables.

Methods: Ten successful model agents were evolved to solve a locomotion task in a $2 \mathrm{D}$ environment containing one stationary object. Their task was to approach and stop at the object. The agents were controlled by a continuous time recurrent neural network (CTRNN). The first layer had five sensory neurons, each accepting a different optical variable (image size, image expansion rate, tau, tau-dot and proportional rate) as input. The second layer was a hidden layer with four neurons. The third layer had two motor neurons controlling acceleration and deceleration. A population-based, real-valued, hillclimbing algorithm was used to evolve the CTRNN parameters. Virtual lesioning analyses were performed to determine the optical variables used by the agents to solve the task.

Results: The performance of all agents was over 97\%. Removing image size and image expansion rate did not change any of the agents' performance. This indicates that none of the agents used these variables. Removing tau and/or proportional rate resulted in a $20 \%-50 \%$ decrease in nine agents' performance. When proportional rate and tau-dot were removed, one agent's performance decreased about $50 \%$.

Conclusion: This work is one of the first studies investigating the informational basis of acceleration together with deceleration in the context of a driving like task. The results indicate that the agents used primarily proportional rate and tau to control acceleration and deceleration even if they had access to five optical variables.

This research was supported by TUBITAK, The Scientific and Technological Research Council of Turkey (Project Number: 114K344).

Keywords: evolutionary robotics, visually-guided braking, control of locomotion, the optic flow, proportional rate

\section{SS2-A04}

The effect of D2 type Medium Spiny Neurons in the striatum

\section{$\underline{\text { Rahmi Elibol }}^{1}$, Neslihan Serap Şengör ${ }^{2}$}

'Department of Electrical and Electronics Engineering, Erzincan Binali YIldırm University, Erzincan, Turkey; ${ }^{2}$ Department of Electronics and Communication Engineering, Istanbul Technical University, Istanbul, Turkey
Objective: This study is carried out to obtain a computational model of striatum, the input of Basal Ganglia (BG) circuits, in cognitive processes and behavior, at the levels of synaptic currents, neuron behaviors and nucleus population.

Methods: In the computational model, neurons and synapse models are obtained considering the synaptic dynamics and behaviors of the Medium Spiny Neurons (MSN) and interneurons (IN) in the striatum. Striatum model is achieved according to the cell and synapse models and whether they are in the dorsal or ventral striatum and the dopamine receptors they hold.

Results: The change of synaptic dynamics and their effect on behavior of MSN and IN cells are obtained as point cells in accordance with the results given in the literature. 3D structure of the nucleus in striatum is modeled using MSNs and INs. The difference between the MSNs having D1 or D2-type dopamine receptors is considered in the modelling. Local field potentials (LFP) are obtained using the synaptic currents of the neurons in the model. By performing frequency analysis, LFP values are obtained and beta frequency band $(13-30 \mathrm{~Hz})$ measured when the dopamine level is low in the striatum is shown with the model.

Conclusion: Starting from the synaptic current level, cell behaviors are obtained according to the nucleus they belong and the receptors they have. In the LFP values, obtained using the synaptic currents and positions of MSN and IN cells, the beta band observed in the striatum is obtained. The source of the beta band is D2- type MSN cells and their activity increases, especially when the dopamine level decreases. The model reveals the integrated role of ventral and dorsal striatum on neural activity. In this work related to modelling of striatum, the components of BG circuit are also considered, so it is possible to observe the role of neuron degeneration.

Keywords: beta frequency band, local field potentials, medium spiny neuron, striatum model

\section{SS2-A05}

Classification of somatic symptom disorder using fNIRS based dynamic functional connectivity

$\underline{\text { Aykut Eken }}^{1}$, Burçin Çolak ${ }^{2}$, Neşe Burcu Bal ${ }^{3}$, Adnan

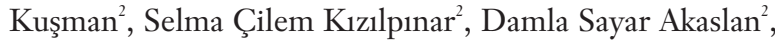
Bora Baskak ${ }^{2}$

'Pompeu Fabra University, Center for Brain and Cognition, Barcelona, Spain; 'Department of Psychiatry, Faculty of Medicine, Ankara University, Ankara, Turkey; ${ }^{3}$ Department of Psychiatry, Health Sciences University, Istanbul, Turkey

Objective: Formerly known as Briquet's syndrome or somatization disorder, the recently re-conceptualized Somatic Symptom Disorder (SSD) is characterized by medically unexplained physical symptoms and related affective and behavioral changes that cause distress and impaired functioning. Its diagnosis mainly depends on careful observation of phenomenology. Although, considerable effort has been put forward to iden- 
tify the physical representation of this disorder, underlying neurological correlates are still unclear.

Methods: In this study, we recruited 21 healthy control and 19 subjects with SSD. We utilized Functional Near-Infrared Spectroscopy (fNIRS) signals, during two painful stimulation; (i) at Individual Pain Threshold (IPT) level and (ii) at Constant Sub-Threshold (CST) level. After performing a region of interest analysis over 18 cortical regions, we estimated dynamic functional connectivity (dFC) over those regions by using sliding window correlation (SWC) method. We extracted features from these time series via these experiments. Then, by using these features as input, we performed automatic classification for SSD and HC approach by using different machine learning algorithms such as Support Vector Machine, Discriminant Analysis and K-nearest neighborhood.

Results: We found highest sensitivity (90\%), specificity (78\%) and accuracy $(85 \%)$ by utilizing connections extracted from dFC maps between Right Superior Temporal- Left Angular Gyri, Right Middle Frontal (MFG) - Left Supramarginal Gyri and Right Middle Temporal - Left Middle Frontal Gyri from IPT experiment.

Conclusion: fNIRS can be used an effective tool and provide robust biomarkers to machine learning algorithms for distinguishing SSD patients from healthy controls and bilateral MFG, left Inferior Parietal, and Right Temporal Gyrus might be considered as critical regions for SSD diagnosis.

Keywords: fNIRS, machine learning, somatic symptom disorder, dynamic functional connectivity, pain

\section{SS2-A07}

Problems of the neuralink project in the horizon of reflexive consciousness theory

Derya Ölcener Ariburnu

Department of Philosophy, Maltepe University, Istanbul, Turkey

Objective: One of the contributions of the philosophy school of German idealism to the field of neuro-philosophy today is the theories developed on consciousness. Consciousness's encounter with the object and the act of reflection that the subject performs between the object and his own mind in this process has become a part of his judgment process. J. G. Fichte's reflexive theory of consciousness shows a triple structure as a self and not an undivided self. Reflection expresses the activity of returning to consciousness itself within the framework of the self, $\sim$ I, the indivisible self. This activity contains clues about how the contents of consciousness can be formed. Then, Hegel discussed the structure of the human mind in detail under the titles of Consciousness, Self-Consciousness, and Reason. Especially in the perception part, the conceptobject and judgment process in the orientation of consciousness to the object at the moment when the subject encounters the object is striking. This process may not only bring about conscious reflexive action but also unconscious acquisitions.
Today, studies on artificial intelligence have gained importance. Elon Musk's Neuralink presentation on August 28, 2020, which connects human and artificial intelligence, brought about a new discussion. The project, which is planned to create an interface between the brain and the machine, includes memory loss, anxiety, addiction, pain, etc. It aims to solve problems. The natural thinking processes of this project subject seem different from the reflection theory. Accordingly, Fichte's not-self and indivisible self-propositions have been replaced by machinery, and has also changed the phenomenology of Hegel. The purpose of this paper is to show how the Neoralink project reversed the theory of reflection.

Methods: Concept research.

Results: In the Neuralink project, the act of reflection changes the human consciousness judgment process.

Conclusion: Problematic aspects of the Neuralink project will be revealed.

Keywords: concious, neuralink, reflection

SS2-B

Nervous System Diseases and Treatment Approaches

\section{SS2-B01}

Nigro-striatal pathway damage on the outcome of spike-and-wave-discharges during epileptogenesis

Melis Yavuz ${ }^{1}$, Yekta Çulpan², Zehra Nur Turgan Aşık², Aylin Toplu², Tuğba Karamahmutoğlu ${ }^{3}$, Medine Gülçebi İdriz Oğlu², Rezzan Gülhan², Filiz Onat ${ }^{2}$

'Department of Pharmacology, Faculty of Pharmacy, Acıbadem Mehmet Ali Aydınlar University, Istanbul, Turkey; ${ }^{2}$ Department of Medical Pharmacology, Faculty of Medicine, Marmara University, Istanbul, Turkey; ${ }^{3}$ Department of Experimental Medical Science, Faculty of Medicine, Lund University, Lund, Sweden

Objective: Certain findings indicate the increase in nigrostriatal dopaminergic activity is inversely proportional to the spike-and-wave discharges (SWDs)-typical absence seizures. The goal is to investigate the effect of nigro-striatal pathway degeneration on SWDs, on the course of absence epilepsy development; namely during epileptogenesis.

Methods: Genetic absence epilepsy rats from Strasbourg (GAERS) of 30 days received $8 \mu \mathrm{g}$ 6-hydroxydopamine (6OHDA) either on medial forebrain bundle (MFB) (single injection; AP: $-1.4 \mathrm{~mm}, \mathrm{ML}:-1.6 \mathrm{~mm}, \mathrm{~V}:-7.1 \mathrm{~mm}$; $\mathrm{n}=3$ ) or striatum (two injections; AP: $-0.5 /+0.5 \mathrm{~mm}, \mathrm{ML}:-3.0 \mathrm{~mm}, \mathrm{~V}$ : $6.0 \mathrm{~mm} ; \mathrm{n}=5)$. Control group recieved saline in the same areas (sham, $\mathrm{n}=6$ ). At the third week, rotation test was performed with apomorphine $(0.05 \mathrm{mg} / \mathrm{kg})$. Sham group and the rotational behavior positive GAERS were implanted with bilateral cortical recording electrodes under ketamine $(100 \mathrm{mg} / \mathrm{kg}$, ip) and xylazine $(10 \mathrm{mg} / \mathrm{kg}$, ip) anesthesia when they were threemonths-old. After one-week-recovery, baseline electroen- 
cephalography were (Powerlab 8S EEG recording system) recorded from the animals and SWD activities were evaluated (Chart 8.0 for Windows program). Two-way ANOVA, Dunnett's post-hoc test were used ( $\mathrm{p}<0.05$; significant).

Results: The cumulative duration $[\mathrm{F}(11.22)=8.413 ; \mathrm{p}<0.001]$ and mean duration of SWDs [F $(11.22)=5.813 ; \mathrm{p}<0.001]$ were statistically significant as a result of 6-OHDA injections applied to the striatum, which was not observed for MFB group. In addition, a distortion in SWD form was observed in the striatum group.

Conclusion: The loss of striatal dopaminergic neurons seem to lead to a disruption in SWD formation. It is known that striatum may be an intermediate step in the generalization of seizures. In light of these findings, it is aimed to evaluate the SWD properties and striatal losses with advanced analysis.

Supported by TUBITAK-SBAG218S653.

Keywords: GAERS, nigrostriatal dopaminergic pathway, spike-and-wave discharges (SWD), epileptogenesis, striatum, 6-OHDA

\section{SS2-B02}

\section{Effects of ghrelin on energy balance on brain tissue of} sepsis-induced rats

Şule Tamer ${ }^{1}$, Elif Özkök ${ }^{2}$, Gülten Ates ${ }^{3}$, Hatice Yorulmaz ${ }^{4}$, Abdullah Aksu', Nuray Balkıs ${ }^{5}$

'Department of Physiology, Istanbul Medical Faculty, Istanbul University, Istanbul, Turkey; ${ }^{2}$ Department of Neuroscience, Aziz Sancar The Institute of Experimental Medicine, Istanbul University, Istanbul, Turkey; ${ }^{3}$ Department of Physiology, Faculty of Medicine, Istanbul Yeni Yüzyıl University, Istanbul, Turkey; ${ }^{4}$ School of Nursing, Haliç University, Istanbul, Turkey; ${ }^{5}$ Department of Chemical Oceanography, Institute of Marine Sciences and Managment, Istanbul University, Istanbul, Turkey

Objective: Sepsis which is defined as increased and irregular response of the host to inflammation and subsequent multiple organ failure has been reported as one of the highest mortality and morbidity rates. Lipopolysaccharide (LPS) is a glycolipid molecule derived from the cell wall of gram-negative bacteria. In studies performed with LPS, it has been reported that as a result of stimulation of sepsis, the blood supply in the brain is impaired and activity is reduced and this situation can cause coma condition due to mitochondrial dysfunction associated with oxidative stress and energy imbalance. Ghrelin, also known as fasting hormone, has several effects on the energy balance and has been shown to be an anti-oxidant peptide. We aimed to investigate the effects of ghrelin on sepsis-induced rats in the brain tissue in energy balance.

Methods: In our study, adult Wistar albino male rats were divided into 4 groups; control: LPS (5 mg/kg i.v., after 12 hours $5 \mathrm{mg} / \mathrm{kg}$ i.p.), Ghrelin (10 nmol/kg i.v.), LPS+Ghrelin. Brain tissues were taken under anesthesia 24 hours after the first injection and homogenized in acid medium. AMP, ADP, ATP, Creatine, Creatine phosphate levels were measured by high performance liquid chromatography method from homogenates. Statistical analysis was performed by one-way analysis of variance and Tukey test.

Results: In the LPS group, AMP, ATP, Creatine and Creatine phosphate levels (respectively; $\mathrm{p}<0.01, \mathrm{p}<0.01, \mathrm{p}<0.05, \mathrm{p}<0.01$ ) were significantly lower than the other experimental groups. ADP levels were significantly high $(\mathrm{p}<0.01)$. There is no significantly difference between the other experimental groups ( $>0.05)$.

Conclusion: We think that the exogenously administered Ghrelin regulates the balance of energy by its anti-oxidant effects via glucose and lipid metabolism deteriorated by stimulating oxidative stress in LPS-induced sepsis.

Keywords: sepsis, brain, ghrelin, energy, LPS

\section{SS2-B03}

\section{Agmatin's ameliorating effect on harmaline-induced} essential tremor

Özlem Akman ${ }^{1}$, Tijen Utkan², Feyza Arıcıoğlu ${ }^{3}$, Nurbay Ateş ${ }^{4}$, Ayşe Karson ${ }^{4}$

'Department of Physiology, Faculty of Medicine, Demiroğlu Bilim University, Istanbul, Turkey; '2Department of Medical Pharmacology, Faculty of Medicine, Kocaeli University, Kocaeli, Turkey; ${ }^{3}$ Department of Pharmacology, Faculty of Pharmacy, Marmara University, Istanbul, Turkey; ${ }^{4}$ Department of Physiology, Faculty of Medicine, Kocaeli University, Kocaeli, Turkey

Objective: Essential tremor (ET), as the most common type of tremor, is a progressive movement disorder with a high prevalence. Although there are a number of options for treatment, the low efficacy of current treatment options reveals the need for new strategies. Harmalin-induced tremor in rats is considered as an ET-model. Although the mechanisms involved in the pathogenesis of ET are not fully known, it has been suggested that it may involve not only glutamate, but also various neurotransmitters such as serotonin, GABA or dopamine. We previously showed that memantine, an NMDA antagonist, suppresses tremor in ET-model. Here, the effects of agmatine, an endogenous neuromodulator with multiple receptor affinity, on tremor were evaluated.

Methods: Adult male Wistar albino rats were used. Harmaline was administered at a dose of $1 \mathrm{ml} / \mathrm{kg}$ (i.p.) to induce ET, and agmatine was administered at a dose of $40 \mathrm{mg} / \mathrm{kg}$ (i.p.) $30 \mathrm{~min}$ utes before harmaline injection to the group, in which its' effect on tremor was evaluated. Tremor duration, intensity (stages 1-5) and locomotion were analyzed in 10-minute consecutive periods with the tremor-locomotion analysis system run under MatLab. The system is based on analysis of signals recorded with dynamic force sensors and images recorded with a color digital camera.

Results: The rats exhibited characteristic tremor patterns at a frequency of $8-12 \mathrm{~Hz}$ in the trunk-head-extremities following harmaline injection. Agmatine reduced the tremor duration 


\section{S112 18th Turkish Neuroscience Congress, 6-9 November 2020, Ankara, Turkey}

during the $2 \mathrm{nd}$ and $3 \mathrm{rd} 10$ minutes $(\mathrm{p}<0.05)$, reduced the stage$4-5$ tremor rate $(\mathrm{p}<0.05)$ during the whole experiment, and reduced the stage-1-2 tremor rate $(\mathrm{p}<0.01)$ during the 2 nd and 3rd 10 minutes after injection. Locomotion, which corresponds to the total distance overtaken during the experiment, decreased in both groups compared to basal activity $(\mathrm{p}<0.01)$.

Conclusion: Our findings suggest that agmatine, an endogenous neuromodulator, suppresses harmaline-induced tremor and may be a potential therapeutic agent in ET therapy.

Keywords: essential tremor, harmaline, agmatine

\section{SS2-B05}

The expression profiles of miR-132, miR-19b and miR-106b in multiple sclerosis patients

Fatma Sağır ${ }^{1}$, Nagehan Ersoy Tunalı ${ }^{2}$ Recai Türkoğlu ${ }^{3}$, Temel Tombul ${ }^{4}$, Erdem Tüzün ${ }^{1}$

'Istanbul University, Department of Neuroscience, Istanbul, Turkey, ${ }^{2}$ Department of Molecular Biology and Genetics, Istanbul Medeniyet University, Istanbul, Turkey; ${ }^{3}$ Department of Neurology, Istanbul Haydarpaşa Numune Training and Research Hospital, Istanbul, Turkey; ${ }^{4}$ Department of Neurology, Göztepe Education and Research Hospital, Istanbul Medeniyet University, Istanbul, Turkey

Objective: Multiple Sclerosis (MS) is an autoimmune inflammatory disease of the central nervous system (CNS), affecting more than 2.5 million people in the world. The disease has multiple subtypes that have distinctive profile of relapse characteristic. Relapsing Remiting MS (RRMS), which consistently deteriorates and involves small remissions, differs from primary progressive MS (PPMS), and in about $60 \%$ of RRMS cases, the disease turns into a progressive form (secondary progressive MS or SPMS). Peripheral blood mononuclear cells (PBMCs) are involved in the pathogenesis of the disease and trigger active demyelination. Micro RNAs (miRNAs) are small non-coding RNA molecules of about 21 nucleotides in length, which regulate gene expression at the post-transcriptional level. MS is considered as the result of an attack of autoreactive PBMCs against myelin antigen and brain is not an easily accessible tissue, both of which make miRNA transcription profiles of PBMC is important. This study investigates the potential of miR-132, miR-19b and miR-106 to be used as biomarkers in the diagnosis and progression of MS.

Methods: In this study, after clinical evaluation of patients with MS, PBMCs were obtained from the blood taken from the donors (6 CIS, 4 PPMS, 12 SPMS, 16 RRMS and 13 healthy controls) and miR-132, miR-19b and miR-106b expression profile analysis was performed with qRT-PCR. Data were analyzed with Mann-Whitney test using Graphpad.

Results: QRT-PCR results shows that miR-132, miR-19b and miR-106b expressions increase in MS patients compared to the healthy controls, and also expressions in PPMS and SPMS subgroups are higher compared to the CIS and RRMS.

Conclusion: Improved understanding of the effects of changes in miR-132, miR-19b and miR-106b is central to more effi- cient MS drug development and will lead to improved therapeutic approaches and targets both as a disease and progression biomarker.

This work is financially supported by Istanbul University Scientific Research Grants (32160).

Keywords: multiple sclerosis, miRNA, biomarkers, PBMC, cytokine

\section{SS2-B06}

Focused ultrasound is a promising cure for AD through its role on the pathobiology of the disease

Merve Beker ${ }^{1}$, Şule Terzioğlu Uşak², Rabia Kevser Boyraz ${ }^{3}$, Shafiga Mursalova Ünal ${ }^{3}$, Erdem Deveci ${ }^{3}$

'Department of Medical Biology, International School of Medicine, University of Health Sciences, Istanbul, Turkey; ${ }^{2}$ Department of Medical Biology, Faculty of Medicine, Bezmialem Vakif University, Istanbul, Turkey; ${ }^{3}$ Department of Psychiatry, Faculty of Medicine, Bezmialem Vakif University, Istanbul, Turkey

Objective: Alzheimer's Disease (AD) is a severe neurological disorder resulting with neuronal death due to accumulation of amyloid beta $(\mathrm{A} \beta)$ plaques. Beyond the approaches for clearance of plaques by pharmacological products, focused ultrasound (FUS) which is the focused beams of ultrasonic energy is a novel technology with a great potential of recovery for $\mathrm{AD}$ patients. The main object of this study was investigating the primary outcomes of FUS treatment on functional and molecular perspectives in a sporadic model of AD.

Methods: 7 months old Long evans rats were infused with $\mathrm{A} \beta$ to the hippocampus by using micro-osmotic pumps. Animals in FUS treatment group were sonicated in a targeted way under a stereotactic frame with a frequency of $2.8 \mathrm{MHz}$ for 10 minutes during 20 successive days. Then animals were tested for memory functions by Morris water maze (MWM) and passive avoidance (PA) behavioral tasks. Later, histological evaluation was made for neuronal survival through cresyl violet staining, for observing plaques through congo red staining and for neuronal degeneration through Fluoro Jade staining. On the molecular level, several important indicators of $\mathrm{AD}$ were also studied by western blot analysis.

Results: Significant reduction in memory skills of $A \beta$ infused animals were observed in PA test. Also, number of surviving neurons in the hippocampus were found to be significantly lower in animals exposed to A $\beta$, whether treated with FUS or not. There was no significant change in the number of degenerated neurons in the hippocampus among the groups. Protein expression studies indicated that, FUS treatment reduced the level of hippocampal pro-apoptotic Bax. Also, upregulated phosphorylation of Tau after $A \beta$ infusion was found to be reversed by FUS treatment.

Conclusion: These results demonstrated that FUS is a promising treatment for $\mathrm{AD}$ through its molecular actions. However, long time observations and detailed functional tests are required for verifying our results. 
Keywords: Alzheimer's disease, focused ultrasound, behavior, survival

\section{SS2-B07}

\section{A novel treatment alternative in cisplatin-induced} neuropathy: low intensity pulsed ultrasound

Büşra Bilir Y1ldız ${ }^{1}$, Özlem Bozkurt Girit ${ }^{2}$, Mehmet Dinçer Bilgin ${ }^{2}$ 'Department of Biophysics, Institute of Health Sciences, Aydin Adnan Menderes University, Aydın, Turkey; '2Department of Biophysics, Faculty of Medicine, Aydın Adnan Menderes University, Aydın, Turkey

Objective: Peripheral neuropathy is a neurological complication that needs an effective treatment strategy and frequently observed due to the use of chemotherapeutic agents. This study aims to investigate the therapeutic efficacy of low frequency pulsed ultrasound (US) on cisplatin-induced peripheral neuropathy.

Methods: Rats were divided into 5 groups as control, cisplatin, melatonin, $1 \mathrm{MHz}$ frequency $0.5 \mathrm{~W} / \mathrm{cm}^{2}$ and $1.5 \mathrm{~W} / \mathrm{cm}^{2}$ US treatment groups. Chemical neuropathy was induced by the intraperitoneal injection of $3 \mathrm{mg} / \mathrm{kg} /$ week cisplatin for 5 weeks (total $15 \mathrm{mg} / \mathrm{kg}$ ). Melatonin group received $10 \mathrm{mg} / \mathrm{kg}$ melatonin (i.p.), and US treatment groups received $0.5 \mathrm{~W} / \mathrm{cm}^{2}$ and $1.5 \mathrm{~W} / \mathrm{cm}^{2}$ power density US at $1 \mathrm{MHz}$ frequency, for 15 days. Nociceptive tests were applied once a week during cisplatin administration and treatment periods. Afterwards, nerve conduction velocities were measured electrophysiologically. Tissue malondialdehyde, superoxide dismutase, interleukin- $1 \beta$ and interleukin-6 levels, thereby oxidative stress and inflammation levels,were determined. Apopototic pathways and the presence of mitophagia were evaluated by caspase-3, bcl-2, and parkin protein levels.

Results: Cisplatin administration led to prolonged nociceptive reponses and decreased nerve conduction velocity, which were recovered by the applied treatments. The increased tissue malondialdehyde and decreased superoxide dismutase levels upon cisplatin administration were observed to be close to those of control levels in the treatment groups. The levels of interleukin- $1 \beta$ and interleukin- 6 were observed to be high in neuropathy group and quite low in the treatment groups. Apoptosis and mitophagy were observed to be suppressed in cisplatin administered groups, whereas applied treatments led to the activation of apoptosis through mitophagy.

Conclusion: Low intensity pulsed ultrasound has been observed to be effective in the treatment of nerve dysfunction in cisplatin-induced peripheral neuropathy, and can be an alternative treatment method to eliminate the complaints of patients with chemical neuropathy.

This study was supported by the Aydin Adnan Menderes University BAP Unit (TPF-19015).

Keywords: peripheral neuropathy, cisplatin, low intensity pulsed ultrasound, melatonin, sciatic nerve function
SS2-C

Behavioral and Cognitive Neuroscience

\section{SS2-C01}

Event-related alpha responses during response inhibition in children and young subjects

Ebru Yıldırım ${ }^{1}$, Tuba Aktürk ${ }^{1}$, Pervin Çalışoğlu², Samet Hakan Uzunlar ${ }^{2}$, Harun Yırıkoğulları ${ }^{3}$, Figen Eroğlu Ada ${ }^{4}$, Enver Atay ${ }^{5}$, Bahar Güntekin ${ }^{6}$, Ömer Ceran $^{5}$

'Program of Electroneurophysiology, Vocational School, Istanbul Medipol University, Istanbul, Turkey; ${ }^{2}$ REMER, Clinical Electrophysiology, Neuroimaging and Neuromodulation Lab., Istanbul Medipol University, Istanbul, Turkey; ${ }^{3}$ Department of Neuroscience, Graduate School of Health Sciences, Istanbul Medipol University, Istanbul, Turkey; ${ }^{4}$ Department of Psychology, Humanities and Social Sciences, Istanbul Medipol University, Istanbul, Turkey; ${ }^{5}$ Department of Pediatrics, School of Medicine, Istanbul Medipol University, Istanbul, Turkey; ${ }^{6}$ Department of Biophysics, School of Medicine, Istanbul Medipol University, Istanbul, Turkey

Objective: Inhibition which is one of the components of the executive functions is a suppression of the responses and/or task-irrelevant cognitive processes. Previous studies reported that age-related neurodevelopmental changes occur in inhibition performance from childhood to adulthood and the changes in inhibition processes are related to changes in the alpha frequency band. To our knowledge, there is no study evaluated the day-night response inhibition in both young subjects and children. Based on this, we aimed to investigate the event-related alpha responses during day-night inhibition task in children and young subjects.

Methods: Fifteen children $(6.73 \pm 0.46)$ and twenty young subjects $(21.95 \pm 2.58)$ were included in the study. EEG was recorded from 18 channels with BrainAmp MR Plus System (sampling rate: $500 \mathrm{~Hz}$; band limits: $0.01-250 \mathrm{~Hz}$ ) during DayNight Stroop task. Power-spectrum and phase-locking analysis were performed for alpha $(8-13 \mathrm{~Hz})$ frequency band. Powerspectrum analysis was performed in three different time-windows [(0-200ms), (200-400ms), (400-600ms)]. Repeated measures of ANOVA was used for statistical analysis $(\mathrm{p}<0.05)$.

Results: Group difference and time-windowXgroup interaction were statistically significant for alpha power (ANOVA; $\mathrm{p}<0.05)$. Whereas the increase of the alpha power (ERS/eventrelated synchronization) in $0-200 \mathrm{~ms}$ has similar for children and youngs, the decrease of the alpha power (ERD/event-related desynchronization) in 200-400 and 400-600 ms was less in children in comparison to young subjects (ANOVA; $\mathrm{p}=0.005$ ). LocationXgroup interaction was significant for alpha phaselocking, the phase-locking of the young subjects had higher than children in frontal, central, and parietal locations (ANOVA; $\mathrm{p}=0.009$ ).

Conclusion: Young subjects had higher alpha ERDs in late time-windows than children and this result demonstrates that 


\section{S114 18th Turkish Neuroscience Congress, 6-9 November 2020, Ankara, Turkey}

motor execution and inhibition processes in young subjects may be more developed in comparison to children. In addition, alpha phase-locking in association areas was higher in young subjects than children. This result shows that cognitive processing in young subjects may be more developed.

Istanbul Medipol University Ethical report no:E30217E30218

Keywords: alpha, children, day-night, EEG, inhibition, youngs

\section{SS2-C02}

Decision-making in aging in light of comparative, computational and neuroscientific perspectives

Ezgi Gür ${ }^{1}$, Yalçın Akın Duyan ${ }^{1}$, Esin Türkakın', Sertan Arkan², Ayşe Karson², Fuat Balc1

'Department of Psychology, Timing and Decision-Making Laboratory, Koç University, Istanbul, Turkey; ${ }^{2}$ Department of Physiology, Faculty of Medicine, Kocaeli University, Kocaeli, Turkey

Objective: Age-related changes in the latent variables of the decision process and their neurobiological correlates have not been studied in the animal models of aging. Given the translational value of animal research, the current study aims to provide a psychomechanistic explanation for these changes using computational modeling and investigate the neurobiological correlates of these age-related alterations in the decision process.

Methods: A two-alternative forced-choice brightness discrimination task was developed and used to test 4- $(\mathrm{n}=11), 10$ $(\mathrm{n}=10)$, and 18-months old $(\mathrm{n}=10)$ C57BL/6J male mice. The latent variables of the decision-making process were examined for different age groups by using the Hierarchical Drift Diffusion Model. Brain slices containing substantia nigra pars compacta, ventral tegmental area (VTA), medial septum/diagonal band complex, and striatum were stained using immunohistochemistry to examine the dopaminergic/cholinergic neurons and/or axon termination densities in these areas and their relations to the model parameters were studied.

Results: The accuracy rate was lower in old compared to young mice $\left[\mathrm{F}(2.28)=5.30, \mathrm{p}=0.01, \eta \mathrm{p}^{2}=0.28\right)$, without any agerelated differences in response times. Modeling results showed that the evidence integration was slower in old compared to young mice $[\mathrm{P}$ (young $>$ old $)>0.95]$. Evidence integration parameter was correlated with the number of dopaminergic neurons in the VTA ( $\mathrm{r}=0.43)$ and the density of dopaminergic axon terminals in the dorsomedial striatum $(\mathrm{r}=0.41)$.

Conclusion: The age-related decrement in the accuracy was due to the slower evidence integration process with aging. Considering the role of the striatal neurons in integrating the information received from the prefrontal cortex and the role of mesocortical dopaminergic input in increasing the signal quality, our results suggest that the age-related slowing in evidence integration process is related to the disrupted mesocortical and nigrostriatal dopaminergic functions.

This study was supported by TUBITAK 2515 (114K991).

Keywords: cognitive aging, dopamine, drift-diffusion model, two-alternative forced-choice

\section{SS2-C03}

Effects of attachment styles on PFC during social interaction: an fNIRS hyperscanning study

Burcu Yargıçoğlu Şahin ${ }^{1}$, Murat Perit Çakır ${ }^{1}$, Bora Başkak ${ }^{2}$

'Department of Cognitive Science, METU Informatics Institute, Ankara, Turkey; '2Department of Psychiatry, School of Medicine, Ankara University, Ankara, Turkey

Objective: As a recently emerging methodology in social neuroscience, hyperscanning have opened up important opportunities for investigating neural correlates of behaviorally observed individual differences such as attachment orientation in realistic social contexts. This study investigates possible differences of prefrontal cortex (PFC) oxygenated hemoglobin ( $\mathrm{HbO})$ activity as well as inter-brain synchronization across PFCs of dyads during naturalistic social interaction from an attachment theory perspective by employing functional nearinfrared spectroscopy (fNIRS) hyperscanning.

Methods: Male participants with right-hand dominance $(n=48)$ were administered Turkish Experiences in Close Relationship Scale (Sumer, 2006) to assess predominant adult attachment style. Regular and reverse cooperative and competitive versions of the well-known rock-paper-scissors (RPS) game were introduced to facilitate standardized social interaction, which dyads were asked to play each RPS version twice facing each other. During each experiment block, participants were asked to play first individually (solo) afterwards with each other (social) 8 rounds of the RPS game variation. fNIRS data were collected from dyads throughout the experiment. By using a novel coherence difference measurement method, which focuses on the difference between solo and social task coherence, significant inter-brain coherence were assessed.

Results: Significant inter-brain coherence were observed for both competition and cooperation tasks at fronto-polar cortex [FC: $\mathrm{t}(14)=3.08, \mathrm{p}<.01, \mathrm{r}=.63$ ] and dorsolateral PFC (DLPFC) bilaterally in a lateralized manner depending on the task type (regular tasks right-DLPFC: $\mathrm{t}(15)=2.85, \mathrm{p}<.01, \mathrm{r}=.59$, reverse tasks left-DLPFC: $\mathrm{t}(13)=3.69, \mathrm{p}<.01, \mathrm{r}=.71)$. Moreover, twosample t-tests based on 27 secure and 21 insecure participants revealed that attachment styles do have effects on $\mathrm{FC} \mathrm{HbO}$ activation (at right FC: cooperation-normal-I $\mathrm{t}(28)=3.77$, $\mathrm{p}<.01, \mathrm{r}=.58$, cooperation-reverse-I $\mathrm{t}(27)=2.66, \mathrm{p}<.05, \mathrm{r}=.45$, competition-reverse-I $\mathrm{t}(28)=2.19, \mathrm{p}<.05, \mathrm{r}=.38)$.

Conclusion: Overall, our findings suggest that FC, while contributing to social interaction, interestingly elicits activation differences w.r.t. attachment styles as well, with secure attach- 
ment orientation exhibiting stronger hemodynamic response, which may serve as a putative neuro-biologic marker underlying individual differences.

Keywords: attachment theory, fNIRS, frontopolar cortex, hyperscanning, prefrontal cortex

\section{SS2-C04}

\section{Investigation of cognitive control in Stroop test by event-related oscillations}

Mehmet Ergen ${ }^{1}$, Emine Elif Tülay ${ }^{2}$

'Department of Physiology, School of Medicine, Acıbadem Mehmet Ali Aydinlar University, Istanbul, Turkey; ${ }^{2}$ Department of Computer Engineering, Faculty of Engineering and Natural Sciences, Üsküdar University, Istanbul, Turkey

Objective: Stroop test is one of the most commonly applied neuropsychological tests. The cognitive performance during Stroop test are argued to be fluctuating during test performance. Previous studies reported decreased accuracy when task neglect is induced by increased occurrence of congruent stimuli, that is suggestive of decreased cognitive control. In the present study we aimed to investigate the cognitive control related components in Stroop test by event-related oscillations (EROs) in EEG.

Methods: 11 healthy volunteers (5 women, 6 men) participated in the study. EEG from 30 electrodes was recorded while standard version (50\% congruent, $50 \%$ incongruent) and taskneglect version ( $80 \%$ congruent, $20 \%$ incongruent) of the Stroop test were applied in 2 blocks. ANOVA was used to assess the behavioral data. EROs were calculated by wavelet transform and assessed by cluster-based nonparametric permutation test implemented in the FieldTrip toolbox.

Results: Reaction times for the incongruent stimuli were longer independent from task neglect condition $(\mathrm{p}=0.025)$. The number of inaccurate responses increased in the task neglect block $(p=0.002)$. The amplitude of total theta $(4-5 \mathrm{~Hz})$ response in 500-600 ms was greater in the incongruent conditions in fronto-central region for both blocks (standard Stroop, $\mathrm{p}=0.002$; task-neglect Stroop, $\mathrm{p}=0.019)$. Evoked delta $(2-3 \mathrm{~Hz})$ response in 500-600 ms was greater in the congruent stimuli conditions in both blocks (standard Stroop, $\mathrm{p}=0.007$; task-neglect Stroop, $\mathrm{p}=0.005)$. This evoked delta response was attenuated in the task neglect Stroop test both for the congruent and incongruent trials $(\mathrm{p}=0.03 ; \mathrm{p}=0.019$, respectively). The amplitude of early evoked theta $(200 \mathrm{~ms})$ in response to incongruent condition in task neglect ( $20 \%$ incongruent) was also attenuated compared with the incongruent condition of standard test (50\% incongruent).

Conclusion: Attenuation of evoked delta and theta responses in the task-neglect Stroop test suggest that these ERO components are associated with the level of the cognitive control during Stroop test performance.

Keywords: EEG, event-related oscillations, stroop test, cognitive control

\section{SS2-C05}

The effect of exercise on memory and VEGF levels in different types of muscle fibers

Aslı Karakılıç ${ }^{1}$, Oğuz Yüksel ${ }^{2}$, Servet Kızıldağ ${ }^{3}$, Ferda Ulviye Hoşgörler ${ }^{4}$, Rabia Ilgınn ${ }^{5}$, Birsu Topçugil ${ }^{2}$, Hikmet Gümüşs ${ }^{6}$ Güven Güvendi', Başar Koç ${ }^{4}$, Sevim Kandişs ${ }^{4}$ Mehmet Ateş ${ }^{3}$, Nazan Uysal ${ }^{4}$

'Department of Physiology, School of Medicine, Balıkesir University, Balıkesir, Turkey; ${ }^{2}$ Department of Sports Medicine, School of Medicine, Dokuz Eylül University, Izmir, Turkey; ${ }^{3}$ College of Vocational School of Health Services, School of Medicine, Dokuz Eylül University, Izmir, Turkey; ${ }^{4}$ Department of Physiology, School of Medicine, Dokuz Eylül University, Izmir, Turkey; ${ }^{5}$ Department of Physiology, Graduate School of Health Sciences, Dokuz Eylül University, Izmir, Turkey; ${ }^{6}$ School of Sport Sciences and Technology, Dokuz Eylül University, Izmir, Turkey; 'Department of Physiology, School of Medicine, Izmir Demokrasi University, Izmir, Turkey

Objective: It is well-known that exercise improves learning and memory via increasing the levels of several growth factors in hippocampus. VEGF is one of the growth factors which is released from muscle tissue during exercise and can cross blood-brain-barrier. The aim of this study was to investigate the relationship between hippocampal memory functions and VEGF levels as well as oxidative exposure in types of muscle fiber in exercised rats.

Methods: 14 Wistar Albino male rats were divided into two groups: (1) Exercise group ( $n=7)$, (2) Control group ( $n=7)$. Rats were run on the treadmill at a speed of $8 \mathrm{~m} / \mathrm{min}$ for the $30 \mathrm{~min}$ utes daily, for 3 days a week for 6 weeks. Memory functions evaluated with Morris-Water-Maze. Their VEGF, Superoxide Dismutase-(SOD), Glutathion Peroxidase-(GPx) and Malondialdehyde-(MDA) levels were measured in type-1 and type-2 fibers using ELISA method.

Results: Learning and memory function is found to be affected significantly positive by exercise $(\mathrm{p}<0.05)$. and VEGF levels of type- 1 and type- 2 muscle fibers were increased by exercise (for both, $\mathrm{p}<0.0001$ ). Muscle VEGF levels were positively correlated with learning and memory test (respectively, type-1; r= $0.691, p=0.0001$, type $-2 ; r=0.716, p=0.0001)$. Exercise reduced SOD and MDA levels in type- $1(\mathrm{p}<0.0001$ and $\mathrm{p}<0.05$, respectively) and type- 2 muscle fibers $(\mathrm{p}<0.002$ and $\mathrm{p}<0.007$, respectively), whereas decreased GPx levels only in type-2 fibers $(\mathrm{p}<0.0001)$.

Conclusion: Our findings suggest that, regular aerobic exercise diminishes the oxidative stress and elevates VEGF levels in both fiber types. VEGF levels produced by both types of muscle fibers appear to be associated with a positive effect of exercise on learning and memory function. These data suggest that VEGF levels induced by both types of muscle fibers may contributes to the positive effects of exercise on learning and memory function

Keywords: exercise, muscle fiber type, VEGF, antioxidant, spatial learning and memory 
S116 18th Turkish Neuroscience Congress, 6-9 November 2020, Ankara, Turkey

\section{SS2-C06}

Effect of stress on winning and losing situations in female badminton players

Müge Sarper Kahveci ${ }^{1}$, Elif Karagün ${ }^{1}$, Sabriye Karadenizli Taşkın², Nurbay Ateş ${ }^{2}$

'Department of Physical Education and Sport, Kocaeli University, Kocaeli, Turkey; ${ }^{2}$ Department of Physiology, Kocaeli University, Kocaeli, Turkey

Objective: In our study, it was planned to investigate the effect of menstruation and stress on the winning/losing situations of athletes.

Methods: 6 of the athletes who faced each other from the women's badminton teams of Yeditepe and Kocaeli universities were asked 11socio-demographic questions, 8 of them from YTU, and then Heart Rate (HR) and Galvanic Skin Responses (GSR) were measured before and after the match. In the control group, the same measurements were applied only once to 15 sedentary university students. During the measurement, raw data of athletes and sedanters were taken for 2 minutes, and then data on the ability to calm down with suggestion was recorded. After the data was first examined with the Shapiro Wilk Test, Independent Groups T-test and Dependent Groups T-test were applied for variables with normal distribution. For those who did not show normal distribution, the Mann Whitney $U$ test and the WilcoxonT-test were applied in the first and last measurement comparisons. Fisher Exact Kikare Test has been tested for categorical variables.

Results: When HR values of the groups were examined, significant differences were found between the first and last measurements made both before and after the match. While the HR values of KOU athletes before the match were significant, there was a significant difference between the HR and GSR measurements ofYTUathletes after the match. When the statistics were examined according to the winner-loser variables, there was a significant decrease in both HR and GSR values of the losing athletes after the match and a significant decrease in the GSR measurements of the winning athletes before the match. According to the menstrual order variable, there was a significant decrease in both HR and GSR measurements after the match of the group with "less regular menstruation". "PreMenstrual Syndrome (PMS)" athletes have significant differences in post-match HR measurements. Finally, both HR and GSR measurements differed significantly between the athlete and controlgroups.

Conclusion: The ability to calm down with pre-match suggestion was more successful in KOU (winning team) athletes, while the ability to calm down with post-match suggestion was more successful in YTU(losing team) athletes. It has shown that YTU athletes have better ability to relax after/the/match. In/addition,it/has/been/clear/that/win/loss,/menstrual/period/andPMS/states/have/an/effect/onHRandGSR/status/of/at hletes.

Keywords: galvanic skin response, heart rate, stress, badminton player, female

\section{SS2-C07}

Effects of age and gender on prospective memory performance and executive functions

İrem Aydınlar ${ }^{1}$, Handan Can ${ }^{2}$

'American Fine Arts and Drama Preschool, Aydın, Turkey; 'Department of Psychology, Bursa Uludağ University, Bursa, Turkey

Objective: The aim of the present study is to examine, age and gender effect on prospective memory and executive functions. Another aim of the study is to examine the relationship between prospective memory and executive functions.

Methods: The sample of this study has been composed of 180 healthy participants which are from three age (20-44, 45-59, 60 and above) and two gender (male, female) levels. Level of education has been balanced in all experimental conditions of this research depending on $3 \times 2$ factorial experimental design. Clinical diagnostic scales, neuropsychological tests (Stroop Test, Wisconsin Card Sorting Test, Trail Making Test, Controlled Oral Word Association Test) and prospective memory tasks (time based-event based) have been applied to all participants during assessment.

Results: A $3 \times 2$ two-way analysis of variance has been applied to all neuropsychological tests. The results indicate that main effect of age over prospective memory and executive function test scores and main effect of gender over Stroop and Trail Making Test scores have been found significant. In addition to these findings, significant interaction effect of age and gender has been found only on Trail Making Test scores. The results also demostrates that, there is a significant relationship between prospective memory task and executive function test scores.

Conclusion: The findings of the present study has indicated that aging individuals has problems with remembering to achieve their plans at the appropriate time and situation. Furthermore, with increasing age executive functions are disturbed parallel to this deteriotation in prospective memory and this leads to problems in the daily functioning of aging people.

Keywords: age, gender, executive function, prospective memory

\section{SS3-A}

Nervous System Diseases and Treatment Approaches

\section{SS3-A01}

Fractional anisotropy changes in the fornix and cingulum tracts in Alzheimer's disease continuum

Gözde Kızılateș Evin ${ }^{1}$, Emre Harı ${ }^{1}$, Ali Bayram², Çiğdem Ulaşoğlu Yıldız', Elif Kurt' ${ }^{2}$, Burak Acar ${ }^{3}$, Tamer Demiralp ${ }^{4}$, İ. Hakan Gürvit ${ }^{5}$

'Neuroimaging Unit, Hulusi Behçet Life Sciences Research Laboratory, Istanbul University, Istanbul, Turkey; 'Department of Neuroscience, Aziz Sancar Institute of Experimental Medicine, Istanbul University, Istanbul, Turkey; ${ }^{3}$ Department of Electrical Electronics Engineering, Faculty of Engineering, Boğaziçi University, Istanbul, Turkey; ${ }^{4}$ Department of Physiology, Istanbul Faculty of Medicine, Istanbul University, Istanbul, Turkey; ${ }^{5}$ Department of Neurology, Behavioral Neurology and Movement Disorders Unit, Istanbul Faculty of Medicine, Istanbul University, Istanbul, Turkey 
Objective: Alzheimer's disease $(\mathrm{AD})$ is a progressive neurodegenerative disease. Diffusion weighted MRI (DWI) changes were observed in disease-specific white matter (WM) tracts in the course of the disease, along which the neurofibrillary tangles spread transsynaptically. WM changes may be important to follow the course of the disease. In this study, the tracts affected in $\mathrm{AD}$ continuum were investigated by using local DWI parameters. Methods: Multi-shell DWI from 15 AD dementia (ADD), 15 mild cognitive impairment (MCI) and 15 subjective cognitive impairment (SCI) subjects were obtained with 3T MRI device at the Neuroscience Unit of the Hulusi Behçet Life Sciences Research Laboratory of Istanbul University. In addition to the $\mathrm{b}=0$ image, DWI data were recorded with 4 different $\mathrm{b}$ values $\left(b=3000 \mathrm{~s} / \mathrm{mm}^{2}, b=1000 \mathrm{~s} / \mathrm{mm}^{2}, b=200 \mathrm{~s} / \mathrm{mm}^{2}, b=100 \mathrm{~s} / \mathrm{mm}^{2}\right)$ from a total of 120 directions. Tract-based spatial statistics (TBSS) based on fractional anisotropy (FA) values was performed using FSL 6.0.3 software (FMRIB Software Library). Three (fornix, ventral and dorsal cingulum) regions of interest (ROI) were determined according to Johns Hopkins University white matter atlas (JHU ICBM-DTI-81). Comparisons between 3 groups were performed by using one-way ANOVA and comparisons between pairs of groups by using t-tests. The significance threshold was set at $\mathrm{p}(\mathrm{FWE})<0.05$.

Results: Significant differences among the groups were obtained in the fornix and right ventral cingulum. FA decreased in the fornix and bilateral ventral cingulum in ADD compared to SCI. In addition, FA decreased in the fornix and right ventral cingulum in ADD compared to MCI.

Conclusion: Our results show that diffusivity of the fornix and ventral cingulum, the main connection tracts of the Papez circuit that builds the neural infrastructure of the episodic memory, are impaired in $\mathrm{AD}$ continuum, and this impairment is associated with clinical severity.

Present work was supported by TÜBİTAK ARDEB (Project Nr:114E053) and IU-BAP (Project Nr: 1567/42362).

Keywords: Alzheimer's disease, mild cognitive impairment, diffusion tensor imaging, tract based spatial statistics

\section{SS3-A02}

\section{Cortical thickness changes in adolescents with obsessive-compulsive disorder}

Öznur Altuntaș ${ }^{1}$, Duygu Kınay ${ }^{2}$, Ulaş Ay ${ }^{3}$, Ali Bayram³ Tamer Demiralp ${ }^{3}$, Murat Coşkun ${ }^{4}$

'Department of Neuroscience, Aziz Sancar Institute of Experimental Medicine, Istanbul, Turkey; ${ }^{2}$ Clinics for Child and Adolescent Psychiatry and Mental Health, Başakşehir, Çam and Sakura City Hospital, Istanbul, Turkey; ${ }^{3}$ Hulusi Behçet Life Sciences Research Laboratory, Neuroimaging Unit, Istanbul University, Istanbul, Turkey; ${ }^{4}$ Department of Child and Adolescent Psychiatry, Istanbul Medical Faculty, Istanbul University, Istanbul

Objective: In search of the neurobiology of obsessive-compulsive disorder (OCD), a number of studies investigated structural changes in the brain; however, there is no consensus on the results. While some of the studies present cortical thinning along with OCD, others report no thickness changes. Additionally, as only a limited number of studies focused on structural changes in adolescents with OCD, we aimed to study cortical thickness changes in adolescent OCD cases.

Methods: 15 adolescents diagnosed with first-episode OCD using DSM-V criteria and 15 age, sex and education matched healthy controls were included. Their T1 weighted magnetic resonance images $(160$ axial slices, gap $=0, F O W=250 \mathrm{~mm}$, voxel size $=1 \mathrm{~mm}$ isotropic, TR/TE/Flip=7700 ms/3800 ms/8 ) taken with $3 \mathrm{~T} \mathrm{MRI}$ scanner were used under the local ethical committee approval (2020-102156). Data analysis is performed with CAT12 (http://www.neuro.uni-jena.de/cat/) software, and cortical thickness values were obtained for each cortical parcel of the DKT40 (Desikan-Killiany-Tourville) atlas (https://surfer.nmr.mgh.harvard.edu/fswiki/CorticalParcellation). Group comparisons with ttests resulting in $\mathrm{p}<0.01$ were reported.

Results: Significant differences were found in 15 anatomical regions between OCD and control groups. Paracentral, postcentral, precuneus, superior parietal gyri displayed bilateral thickening in adolescent OCD group compared with the healthy adolescents. Additionally, unilateral increases in cortical thickness were observed in right precentral, right inferior parietal, left pars orbitalis, left superior frontal, left caudal middle frontal, and left supramarginal gyri.

Conclusion: The overlap between areas with increased thickness in adolescent OCD in the present study and those with reduced thickness reported in adult OCD studies in the literature provides an interesting perspective on the neurodevelopmental aspect of the disorder. Furthermore, cortical thickness differences we observed in posterior areas such as precuneus, cuneus, right inferior parietal cortex in addition to areas of motor and executive functions, support recent OCD studies that claim the role of posterior association areas in the pathogenesis of the disease.

Present work was supported by IU-BAP (project \#TU201721107).

Keywords: magnetic resonance imaging, obsessive-compulsive disorder, cortical thickness

\section{SS3-A03}

\section{Functional connectivity changes of default mode and} salience networks in Alzheimer's disease

Elif Kurt ${ }^{1}$, Emre Harı ${ }^{2}$, Kardelen Eryürek ${ }^{2}$, Çiğdem Ulaşoğlu Yıldız 2 , Elif Yıldırım³, Ezgi Soncu Büyükişcan ${ }^{4}$, Burak Acar ${ }^{5}$, Hakan Gürvit ${ }^{6}$, Tamer Demiralp ${ }^{7}$

'Department of Neuroscience, Aziz Sancar Institute of Experimental Medicine, Istanbul University, Istanbul, Turkey; ${ }^{2}$ Neuroimaging Unit, Hulusi Behçet Life Sciences Research Laboratory, Istanbul University, Istanbul, Turkey; ${ }^{3}$ Department of Psychology, Isik University, Istanbul, Turkey; ${ }^{4}$ Department of Psychology, Yeditepe University, Istanbul, Turkey; ${ }^{5}$ Department of Electrical \& Electronics Engineering, Bogazici University, Istanbul, Turkey; ${ }^{6}$ Department of Neurology, Faculty of Medicine, Istanbul University, Istanbul, Turkey; 'Department of Physiology, Faculty of Medicine, Istanbul University, Istanbul, Turkey 
Objective: The intrinsic connectivity networks mostly affected in Alzheimer's disease (AD) are the default mode (DMN) and salience networks (SN). The capacity of the functional connectivity (FC) changes in these networks in differentiating subjective cognitive impairment (SCI), earliest stage of cognitive decline along the $\mathrm{AD}$ continuum, from objectively diagnosed mild cognitive impairment (MCI) is not yet shown. Therefore, we investigated FC changes in DMN and SN among SCI, MCI and AD dementia (ADD) groups.

Methods: Resting-state fMRI data of 88 participants (21 ADD, 34 MCI, 33 SCI) were collected with 3T MRI scanner. FC of 11 regions of interest (ROI) corresponding to DMN and $\mathrm{SN}$ nodes were analysed using CONN-toolbox (https://web.conn-toolbox.org/). Cluster level significance threshold in the F test among the 3 groups was set at $\mathrm{p}(\mathrm{FWE}-\mathrm{corr})<0.0045$ (Bonferroni corrected according to number of ROIs). For ROIs with significant difference in the $\mathrm{F}$ test, $\mathrm{t}$-tests were performed between pairs of groups, and results with $\mathrm{p}(\mathrm{FWE}$-corr $)<0.017$ were reported.

Results: In ADD compared to both MCI and SCI, FC of posterior cingulate cortex node of DMN with temporal and occipital cortices, FC of anterior cingulate cortex (ACC) node of SN with right insula and temporal cortices and $\mathrm{FC}$ of right and left anterior insula nodes of SN with ACC significantly decreased. Additionally, for ADD vs MCI comparison, FC of left anterior insula node of SN with the right insula, and for ADD vs SCI comparison, FC of the right and left anterior insula nodes of $\mathrm{SN}$ with the supplementary motor area were reduced.

Conclusion: The findings reveal that the FC changes of DMN and $\mathrm{SN}$ in $\mathrm{AD}$ continuum occur in the advanced stages of the disease with no distinctive change between SCI and MCI stages.

Present work was supported by TÜBİTAK (Project Nr: 114E053) and IU-BAP (Project Nr:1567/42362).

Keywords: Alzheimer's disease, default mode network, salience network, functional connectivity, magnetic resonance imaging

\section{SS3-A05}

Comparing short-term memory, working memory and executive functions among patients with relapsing remitting multiple sclerosis and healthy individuals

Furkan Duman ${ }^{1}$, Handan Can ${ }^{2}$, Alev Leventoğlu ${ }^{3}$

'Department of Psychology, Ufuk University, Ankara, Turkey; ${ }^{2}$ Department of Psychology, Bursa Uludağ University, Bursa, Turkey; ${ }^{3}$ Department of Neurology, Ufuk University, Ankara, Turkey

Objective: Multiple sclerosis causes physical and cognitive impairments. However, the studies conducted to evaluate the cognitive functions of MS patients had controversial findings. The aim of the current study was to compare the short-term memory, working memory and executive functions of patients with Relapsing Remitting Multiple Sclerosis (RRMS) and healthy individuals.
Methods: The sample of the study consisted of 25 RRMS patients and 25 healthy participants. The groups were matched for age, gender, education level and hand preference. Data were collected through self-report questionnaires and neuropsychological tests including "Demographic Information Form", "Visual Aural Digit Span Test B Form", "Wisconsin Card Sorting Test (WCST)", "Backward Digit Span Task", "Stroop Test T-BAG Form", "WMS-R: Visual Memory Span Subtest", and "Trail Making Test (TMT)".

Results: The analysis showed that RRMS patients made less conceptual level responses (WCST) than healthy individuals $(\mathrm{U}=192.50, \mathrm{Z}=-1.97, \mathrm{p}<.05)$. The RRMS patients were also slower than healthy individuals in terms of TMT part A ( $\mathrm{U}=91.50, \mathrm{Z}=-2.60, \mathrm{p}<.01)$ and Stroop Test T-BAG Form Part 3 ( $U=200.50, Z=-2.20, p<.05)$. Furthermore, RRMS patients' forward visual memory span $(\mathrm{U}=179.50, \mathrm{Z}=-2.69, \mathrm{p}<.01)$ and backward visual memory span $(\mathrm{U}=174.50, \mathrm{Z}=-2.82, \mathrm{p}<.01)$ which were obtained from WMS-R Visual Memory Subtest were lower than healthy individuals

Conclusion: The results showed that visuo-spatial short-term and working memory spans of the RRMS patients were significantly lower than healthy individuals. However, verbal shortterm and working memory spans did not differ significantly from healthy individuals. When the results obtained from the tests that are sensitive to executive functions were examined, it was seen that the RRMS group had significantly lower conceptualization skills compared to the control group, while there was no significant difference between the groups in terms of perseveration, inhibition and set shifting skills.

Keywords: multiple sclerosis, short-term memory, working memory, executive functions, neuropsychological assessment

\section{SS3-A06}

Regenerative capacity of mesenchymal stem cells in peripheral nerve injury crush model

Ramazan Üstün ${ }^{1}$, Elif Kaval Oğuz ${ }^{2}$, Tunç Akkoç ${ }^{3}$, Ayşe Şeker ${ }^{1}$, Rabia Sena Türker ${ }^{4}$, Siddık Keskin ${ }^{5}$

'Department of Physiology, School of Medicine, Van Yüzüncü Yıl University, Van, Turkey; 'Unit of Neuroscience Research, School of Medicine, Van Yüzüncü Yıl University, Van, Turkey; ${ }^{3}$ Department of Pediatrics, School of Medicine, The Division of Allergy and Immunology, Marmara University, Istanbul, Turkey; ${ }^{4}$ Department of Pharmacology, Faculty of Pharmacy, Van Yüzüncü Yıl University, Van, Turkey; ${ }^{5}$ Department of Biostatistics, School of Medicine, Van Yüzüncü Yıl University, Van, Turkey

Objective: Current study aimed to elucidate the regenerative capacity of mesenchymal stem cells (MSC) in a peripheral nerve crush model.

Methods: Study design was composed of control ( $\mathrm{n}=7)$, crush $(n=7)$, crush + MSC (treatment) $(n=7)$ groups. Sciatic nerve crush injury model was established in adult male mice. Bone marrow-derived MSC (800,000 cells) was administered to the 
treatment group with a single intraperitoneal dose. The study was continued for eight weeks. Degeneration and regeneration findings were obtained by functional tests and immunohistochemical techniques. Sensory function test (hotplate/pain nociception latency) and motor function test (sciatic function index) were used for functional evaluation. Immunofluorescence imaging of nerve and target muscle tissue was performed for immunohistochemical evaluation. Axonal markers (neurofilament 200 (NFH), $\beta$ III tubulin), myelin markers (MBP, MP0) and nerve-muscle junction markers (neurofilament 200, $\alpha$ bungarotoxin $(\alpha-B T X)$ ) were used. Immunoreactivities of proteins were analyzed with Image J software.

Results: Functional tests performed one week after crush injury showed that the injured groups suffered significant sensory and motor function loss compared to the control group $(\mathrm{p}<0.05)$. The tests repeated every week throughout the study showed that the MSC treatment group's functional activity was higher than the crush group $(\mathrm{p}<0.05)$, and there was no difference between the control group and the MSC group. In immunohistochemical comparison, immunoreactivities of axonal structures ( $\beta$ III tubulin, NFH) were significantly higher in control and MSC groups than the crush group $(\mathrm{p}<0.05)$; there was no difference between the control group and MSC group. Immunreactivities of myelin proteins (MBP, P0) were higher in the control group than the other groups $(\mathrm{p}<0.002)$; there was no difference between the crush group and the MSC group. In the immunohistochemical comparison of target muscle tissue, NFH immunoreactivity showed a significant difference; it was the highest in the control group, the MSC group was moderate, and the crush group was the lowest $(\mathrm{p}<0.002)$. There was no difference between the groups in the immunoreactivity of $\alpha$-BNG.

Conclusion: MSC treatment carried out the regeneration of the axonal structures of the sciatic nerve. There were signs of healings in myelin and neuromuscular junctions but were not statistically significant. In new studies for the recovery of myelin and target muscle group, the second MSC treatment dose should be tried, and the study should be planned for a more extended period.

This study was supported by the Van YYÜ Scientific Research Projects Coordination Unit (Project number: 2015-TF-B282).

Keywords: mesenchymal, peripheral, nerve injury, degeneration, regeneration

\section{SS3-A07}

\section{The importance of hippocampus neuroanatomy in epilepsy surgery}

Ayşegül Esen Aydın

Department of Neurosurgery, Bakırköy Research and Training Hospital for Neurology, Neurosurgery and Psychiatry, Istanbul, Turkey

Objective: The hippocampus plays an important role in learning and memory. This was first demonstrated by the famous

patient HM who underwent bilateral temporal lobectomy for treatment of intractable epilepsy. Subsequently, the hippocampus became the focus in studies examining the pathophysiology of memory disorders. The hippocampus is highly susceptible to damage caused by hypoxia, ischemia and encephalitis. As the most electrically excitable region of the brain, it is a major source of epileptic seizures. In this study, we aimed to demonstrate the important anatomical structures encountered during epilepsy surgery.

Methods: Four formalin-fixed human brain hemispheres kept in $10 \%$ formalin solution for at least 2 months were used. After removal of arachnoid mater, pia mater and vascular structures, hemispheres were frozen at $-16^{\circ} \mathrm{C}$ for at least 2 weeks. Brain hemispheres were kept in $70 \%$ alcohol solution at room temperature between dissection sessions.

Results: The hippocampus is located with the choroid plexus within the lateral ventricle temporal horn. It is divided macroscopically into 3 parts as body, head and tail. The body is a convex structure covered with alveus. Alveus is a subependymal fiber layer. The body and tail of the hippocampus are adjacent to the choroidal fissure. The bottom end of the choroidal fissure is the inferior choroidal point and is a landmark just behind the head of the hippocampus to which the choroid plexus attaches.

Conclusion: Understanding of the neuroanatomical points that constitutes the hippocampectomy steps is very important for surgery. The steps of opening the choroidal fissure, releasing the head of the hippocampus, and medial and posterior disconnection are shown in our study. A better understanding of the anatomical features and boundaries of the hippocampus will help to prevent the possible damage and related complications as well as to ensure the success of epilepsy surgeries in this region.

Keywords: epilepsy surgery, hippocampus, neuroanatomy

SS3-B

Behavioral and Cognitive Neuroscience

\section{SS3-B01}

Investigation of Alzheimer's disease and white matter mass change relationship with machine learning

Emine Petekkaya ${ }^{1}$, Zülal Kaptan ${ }^{2}$

'Department of Anatomy, Faculty of Medicine, Kastamonu University, Kastamonu, Turkey; 'Department of Physiology, Faculty of Medicine, Beykent University, Istanbul, Turkey

Objective: Using machine learning algorithms for disease risk prediction has become an important new approach. In previous studies, a remarkable reduction in white matter volume and degeneration of tract fibers has been observed in Alzheimer's disease $(\mathrm{AD})$. Our aim was to investigate the relationship of 
white matter tracts with the risk of developing $\mathrm{AD}$ by using machine learning algorithms.

Methods: 3D magnetic resonance images were provided for volumetric analysis of gray and white matter regions from 26 healthy and $21 \mathrm{AD}$ subjects and converted to the format to be loaded on Mricloud system. Volume analysis of 294 tissues of white matter was done. In Spyder environment with Anaconda distribution using Python 3.7, data was classified by logistic regression and the predictive power of the model was tested. Then the importance test was done. The arithmetic means of the tracts that seemed to have high importance were compared between the groups in SPSS software.

Results: The model created had 91\% accuracy score. Importance test showed 13 tracts highly associated with $\mathrm{AD}$ (10 hypertrophies, 3 atrophies). When the arithmetic means were compared, significant differences were found between groups. In the temporal lobe; atrophy was observed in the subcortical fibers of the left gyrus temporalis superior pole $(\mathrm{p}<0.05)$ and left gyrus temporalis medius $(\mathrm{p}<0.001)$ regions, while hypertrophy was observed in the right gyrus temporalis superior fibers $(\mathrm{p}<0.05)$. There was also hypertrophy in the left fornix $(\mathrm{p}<0.05)$ and left cingulum $(\mathrm{p}<0.01)$ region.

Conclusion: In the prodromal phase of $\mathrm{AD}$, the posterior region of the cingulum and thalamus are of potential importance. Hippocampal inputs reach the thalamus via fornix. Hypertrophy observed in left fornix and left cingulum region of these tracts can be associated with episodic memory problem due to connection disorder. Our results have revealed important results associated with early pathological indicators of AD.

Keywords: Alzheimer's disease, magnetic resonance imaging, white matter, tract fibers, machine learning

\section{SS3-B02}

Investigation of emotional working memory in Alzheimer type dementia using f-NIRS

Fatma Ebru Köse ${ }^{1}$, Banu Cangöz ${ }^{2}$, Erguvan Tuğba Özel Kızıl’, Bora Baskak ${ }^{3}$ Zeynel Baran², Halise Devrimci Özgüven ${ }^{4}$

'Department of Psychology, Faculty of Medicine, Aydın Adnan Menderes University, Aydın, Turkey; ${ }^{2}$ Department of Psychology, Faculty of Medicine, Hacettepe University, Ankara, Turkey; ${ }^{3}$ Department of Psychiatry, Faculty of Medicine, University of Ankara, Ankara, Turkey; ${ }^{4}$ Brain Research Center, Ankara University, Ankara, Turkey

Objective: Emotional working memory (EWM) is suggested as a working memory (WM) type, distinguished to process emotional stimuli, and may or may not be spared in Alzheimer's disease (AD). The aim was to compare patients with $\mathrm{AD}$ and healthy older adults $(\mathrm{HC})$ on verbal EWM performance and accompanying prefrontal cortex activity.

Methods: Twenty AD patients along with $20 \mathrm{HC}$ individuals are required to complete an emotional one-back task in three conditions (neutral, positive and negative word lists). Prefrontal oxyhemoglobin (oxyHb) concentrations were measured simultaneously by a 24- channel functional near infrared spectroscopy device.

Results: Correct response rates were similar in two groups in all conditions. Reaction times were comparable in the EWM positive condition but longer in the $\mathrm{AD}$ group in EWM neutral and negative conditions. In the HC group, emotional words had no significant effect on WM. On the other hand, positive compared to neutral words led to greater activation in the left ventral prefrontal cortex (VPFC) in $\mathrm{AD}$ group. When compared to HCs, activity in the VPFC was significantly higher in $\mathrm{AD}$ patients during the positive condition

Conclusion: Positive words facilitated WM performance in participants with AD. Activity in VPFC may be the functional correlate of this phenomenon.

Keywords: Alzheimer type dementia, working memory, emotion, n-back task, prefrontal cortex, positivity effect

\section{SS3-B03}

\section{A longitudinal study of saccadic eye movements in patients with mild cognitive impairment}

Müge Akkoyun $^{1}$, Koray Koçoğlu' ${ }^{1}$, Gülden Akdal ${ }^{2}$

'Department of Neurosciences, Institute of Health Sciences, Dokuz Eylül University, Izmir, Turkey; ${ }^{2}$ Department of Neurology, Faculty of Medicine, Dokuz Eylül University, Izmir, Turkey

Objective: This study aims to evaluate longitudinal changes in saccadic eye movements of patients with mild cognitive impairment (MCI) and healthy controls (HCs) and its relationship with changes in cognitive functions.

Methods: The study included 19 MCI patients and 15 HCs. Patients with MCI consist of 12 amnestic MCI (aMCI) and 7 non-amnestic MCI (naMCI) form. A neuropsychological test battery and eye movement paradigms were applied to participants in a time period of $24.9 \pm 2.2$ months. The variation between the two evaluations was examined by measuring latency, anti-saccade error rate, express anti-saccade rate and anticipatory anti-saccade rate. The relationship between cognitive functions and changes in eye movements was evaluated.

Results: Three aMCI patients progressed to Alzheimer's disease and one HC progressed to naMCI. It was observed that anti-saccade latency significantly decreased in the aMCI group ( $p=0.042$ ), while the anti-saccade latency was significantly increased in HCs $(\mathrm{p}=0.040)$. In cognitive functions, a significant decrease was observed in the means of Mini-Mental State Examination results in aMCI $(\mathrm{p}=0.010)$ and naMCI $(\mathrm{p}=0.045)$ groups. Negative correlation was found between changes in executive functions and horizontal pro-saccade latency in naMCI group $(\mathrm{r}=-0.829, \mathrm{p}=0.042)$. There was a negative correlation between changes in language and anti-saccade error rate in the aMCI group $(\mathrm{r}=-0.699, \mathrm{p}=0.011)$. In HCs, negative correlation was found between changes in executive functions and anticipatory anti-saccade rate $(r=-0.600, p=0.023)$. 
Conclusion: It has been observed that changes in eye movements of MCI patients and HCs may be related to cognitive functions. Increased latencies of HCs may be a predictor of future cognitive dysfunction. The importance of longer longitudinal follow-up was emphasized due to the participants in which the change in the course of the disease was observed.

Keywords: mild cognitive impairment, eye movements, neuropsychological assessment, longitudinal study

\section{SS3-B04}

Evaluation of the effectiveness of virtual reality based cognitive training with EEG in Individuals with mild cognitive impairment: preliminary results

Yağmur Özbek $^{1}$, İlayda Kıyı ${ }^{1}$, Deniz Yerlikaya ${ }^{1}$, Markus Berger $^{2}$, Faik Kartelli², Köksal Alptekin ${ }^{3}$, Görsev G. Yener ${ }^{4}$

'Department of Neurosciences, Institute of Health Sciences, Dokuz Eylül University, Izmir, Turkey; ' Department of Film Design Animation Film Design and Directing, Faculty of Fine Arts, Dokuz Eylül University, Izmir, Turkey; ${ }^{3}$ Department of Psychiatry, School of Medicine, Dokuz Eylül University, Izmir, Turkey; ${ }^{4} / z m i r$ Biomedicine and Genome Center, Dokuz Eylül University Health Campus, Izmir, Turkey

Objective: Mild cognitive impairment (MCI) is an intermediate stage characterized by cognitive impairment between healthy aging and dementia. Yet, there is not a pharmacological treatment of MCI. Virtual reality (VR)-based CT used for cognitive rehabilitation has a high ecological validity and promising results. This study aimed to investigate the efficacy of VR-based CT in MCI.

Methods: This study included 6 individuals with MCI. VRbased CT includes two bazaars which consist of real scenes recorded by $360^{\circ}$ cameras. VR-based CT has applied for 2 sessions per week a total of 16 sessions for 8 weeks with VR-goggles and joysticks. The sessions were including tasks which aim to improve memory, attention, decision-making and visuospatial abilities. Neuropsychological tests and EEG recordings with an auditory oddball paradigm were applied pre-CT and post-CT. Independent Component Analysis (ICA) were performed to correct ocular artifacts and the remaining artifacts were eliminated automatically. Mean P300 amplitudes for target stimuli were automatically measured from $\mathrm{Fz}, \mathrm{Cz}, \mathrm{Pz}$ electrode locations in the $250-550 \mathrm{~ms}$ time window.

Results: Wilcoxon Test showed a significant difference in $\mathrm{Cz}$ $(\mathrm{Z}=-1.992, \mathrm{p}=0.046)$ and $\mathrm{Pz}(\mathrm{Z}=-1.992, \mathrm{p}=0.046)(\mathrm{Z}=-1.992$, $\mathrm{p}=0.046$ ) electrode locations between pre- and post-CT indicating individuals with MCI had higher P300 amplitudes after VR-based CT.

Conclusion: It is known that P300 amplitudes are associated with cognitive functions, especially memory and attention. The increased P300 amplitudes may relate to improvement in cognitive functions. Further studies should investigate the efficacy of VR-based CT by using a control group and a larger sample.

This study was supported by Dokuz Eylül University Department of Scientific Research Projects (Project Number:2016.KB. MLT.003)
Keywords: mild cognitive impairment, virtual reality, cognitive training, P300

\section{SS3-B05}

Behavioral and cognitive outcomes of being born to obese parents in rats

Enver Ahmet Demir ${ }^{1}$, Gülay Gülbol Duran², Meral Urhan Küçük ${ }^{2}$, Hatice Doğan ${ }^{1}$, Okan Tutuk ${ }^{1}$, Funda Çimen ${ }^{3}$, Mucella Bayirli', Cemil Tumer ${ }^{1}$, Nizami Duran ${ }^{3}$

'Department of Physiology, Faculty of Medicine, Hatay Mustafa Kemal University, Hatay, Turkey; ${ }^{2}$ Department of Medical Biology, Faculty of Medicine, Hatay Mustafa Kemal University, Hatay, Turkey; ${ }^{3}$ Department of Microbiology and Clinical Microbiology, Faculty of Medicine, Hatay Mustafa Kemal University, Hatay, Turkey

Objective: Obesity is a growing health problem in developed countries. We investigated transgenerational behavioral/cognitive outcomes of parental obesity in offspring rats.

Methods: Animals born to standard diet-fed parents were fed on either standard $(\mathrm{Cnt})$ or an obesogenic diet $(\mathrm{Ob} / \mathrm{P})$ for 12 weeks. Afterwards, Ob/P animals were left to mate. Pups were also received obesogenic $(\mathrm{Ob} / \mathrm{O})$ or standard diet $(\mathrm{Std} / \mathrm{S})$ until the 12 th post-weaning week.

Results: In females, the locomotor activity was increased in all groups compared to Cnt in the open-field test, but in males. The time consumed in the open arms of the elevated-plus maze was found higher in $\mathrm{Ob} / \mathrm{P}$ than other groups in females, and it was higher in $\mathrm{Ob} / \mathrm{P}$ and $\mathrm{Ob} / \mathrm{S}$ than $\mathrm{Cnt}$ in males. The time elapsed with mobile behaviors in the forced swim test was longer in $\mathrm{Ob} / \mathrm{P}$ and $\mathrm{Std} / \mathrm{S}$ compared to Cnt in both sexes. In the Barnes maze test, time passed in the target quadrant was lower in all groups than Cnt in females. In males, it was lessened only in $\mathrm{Ob} / \mathrm{P}$ compared to Cnt. The three-way ANOVA revealed that "diet" or "parental obesity", and the interaction among "diet and parental obesity" or "parental obesity and sex" were influencers of memory.

Conclusion: These results demonstrated that parental (maternal\&paternal) obesity alters behaviors and cognition in the offspring. Being born to obese parents increases voluntary locomotor activity particularly in females. Anxiolytic effects, which were unrelated to parental obesity, were pronounced with the obesogenic diet. Antidepressant-like effects that were manifested with obesogenic diet in sex-separated comparisons were found to be apparent in the pups born to obese parents (and fed on standard diet) only when a sex- combined comparison was performed. Lastly, we noted that the consumption of an obesogenic diet is the most prominent factor that disturbs memory, although parental obesity and sex are additional influencers. (Supported by TUBITAK-3501 \#118S404)

Keywords: anxiety, cognition, depression, locomotion, offspring rat, parental obesity 


\section{SS3-B06}

Evaluation of cognitive functions in patients with acute and chronic unilateral vestibulopathy

Dilara Aktert Ayar ${ }^{1}, \underline{\text { Kübra Celik }}{ }^{2}$, Emre Kumral $^{1}$, Neşe Çelebisoy

'Department of Neurology, School of Medicine, Ege University, Izmir, Turkey; ${ }^{2}$ Department of Neuroscience, Institute of Health Sciences, Ege University, Izmir, Turkey

Objective: The study aims to evaluate the cognitive functions of patients with acute and chronic unilateral vestibular (UVL) loss compared to healthy controls.

Methods: Cognitive tests assessing visuospatial functions via Benton's Judgment of Line Orientation Test (BJLO), Cancellation Tests (CT), Rey-Osterrieth Complex Figure Test (ROCF) and verbal memory, retention of information via Oktem Verbal Memory Process Test (OVMP), Forward and Backward Digit span (DS) were used in addition to Beck depression and Anxiety inventories for 21 patients diagnosed with UVL. After at least 6 months, the same tests were repeated to 8 of 21 patients. SPSS Statistics 25.0 was used for statistical analysis and $\mathrm{p}<0.05$ was considered.

Results: 12 male, 9 female (age $54.3 \pm 10.3$ ) patients and 13 male, 7 female (age 49.3 \pm 8.5 ) healthy controls participated in the first study. Abnormalities in CT, BJLO and backward DS were found $(\mathrm{p}<0.05)$. A very prominent difference regarding Beck depression $(\mathrm{p}<0.05)$ and anxiety inventories $(\mathrm{p}<0.001)$ was present. OVMP, CT and BJLO scores recorded 6 months later from 7 male and 1 female patients (age $53.13 \pm 11.837$ ) were still lower than the controls. However, when the first and second test results of the patients were compared, there was an increase in OVMP, Backward DS, ROCF - Immediate and Delayed Recall scores, while the trial time was significantly low $(\mathrm{p}<0.05)$.

Conclusion: Deficits in visuospatial functions and short term memory detected in our patients with acute UVL seem to be enhanced by accompanying anxiety and depression. After a while, verbal memory and visuospatial attention of the patients improved. However, when compared with the controls, the results were still worse and patients were still more prone to anxiety.Higher patient numbers are needed to achieve more reliable results in further studies.

Keywords: unilateral vestibulopathy, cognition, memory, anxiety

\section{SS3-B07}

Effects of forced nicotine application and deprivation, on behavior and neuropeptide $Y$ mRNA expression

Fulya Tuzcu $^{1}$, Ramazan Can Gökmen ${ }^{1}$, Ali Birdoğan², Meliha Öztürk ${ }^{1}$, Buker Aypar ${ }^{1}$, Ayşegül Keser ${ }^{1}$, Lütfiye Kanıt ${ }^{1}$, Ersin Oğuz Koylu, Burcu Balkan¹, Oğuz Gözen ${ }^{1}$

'Department of Physiology, Medical School, Center For Brain Research, Ege University, Izmir, Turkey; '2Department of Neuroscience, Institude of Health Sciences, Ege University, Izmir, Turkey

Objective: Neuropeptide Y (NPY) plays an important role in addiction, stress response and anxiety-related mechanisms.
NPY can increase or decrease anxiety through different receptors. This study aims to investigate behavioral changes during nicotine reward/abstinence and the regulation of NPYmRNA in mesocorticolimbic system.

Methods: Wistar rats were divided into 4 groups $(\mathrm{n}=10)$ : control, reward, 24-hour withdrawal (24W), 48-hour withdrawal $(48 \mathrm{~W})$. For twelve weeks, only drinking water with $50 \mu \mathrm{g} / \mathrm{ml}$ nicotine (free base) was offered to groups except the control. The elevated plus maze (EPM) and somatic withdrawal signs test were performed at the 13th week. After the mesocorticolimbic areas were dissected, NPYmRNA expression was measured by qRT-PCR method. The results were evaluated by oneway ANOVA and post-hoc tests.

Results: The closed arm entry latency, which was considered as a decrease in anxiety in the EPM test, increased in the reward group and decreased in the $24 \mathrm{~W}$ group $(\mathrm{p}<0.05)$. Locomotor activity and escape-type behaviors increased in the reward group, explaining the locomotor activator effect of nicotine $(\mathrm{p}<0.01$ and $\mathrm{p}<0.05)$, and decreased in the $24 \mathrm{~W}$ group $(\mathrm{p}<0.05)$. Gasping, an important sign of withdrawal, increased only in the $24 \mathrm{~W}$ group $(\mathrm{p}<0.05)$. NPYmRNA increased in PFC $(\mathrm{p}<0.001)$ and amygdala $(\mathrm{p}=0.027)$ in the $24 \mathrm{Y}$ and $48 \mathrm{Y}$ groups, while it increased in the reward group in the hippocampus $(\mathrm{p}=0.007)$. There was no significant change in other brain regions.

Conclusion: With nicotine, an increase in locomotion and decrease in anxiety levels were detected, while a decrease in locomotion and increase in anxiety levels were detected in withdrawal. Our research has shown that NPYmRNA expression is differently regulated in different regions of the mesocorticolimbic system during nicotine reward and withdrawal. Our findings support that changes in NPYmRNA expression may have regulatory effects on mood in nicotine reward or withdrawal.

Our research was supported by Ege University BAP Coordination Office (18-BAUM-001, EÜHADYEK 2017-101).

Keywords: nicotine, neuropeptide Y, abstinence, withdrawal symptoms, elevated plus maze

\section{SS3-C}

Neural Function, Sensory and Motor Systems

\section{SS3-C01}

\section{Modulation of cutaneous silent period}

Uygur Tanrıverdi $^{1}$, Ayşegül Gündüz ${ }^{1}$, Hatice Kumru ${ }^{2}$, Meral Erdemir Kiziltan ${ }^{1}$

'Department of Neurology, Cerrahpaşa Medical Faculty, Istanbul UniversityCerrahpaşa, Istanbul, Turkey; 'Institut Guttmann Neurorehabilitation Institute, Badalona, Spain \& Fundació Institut d'Investigació en Ciències de la Salut Germans Trias i Pujol, Badalona, Spain

Objective: Cutaneous silent period (CSP) is a spinal inhibitory reflex, which is generated at spinal level and modulated by supraspinal structures. Here, we aim to study modulation of CSP in healthy subjects. 
Methods: We included 10 healthy volunteers (ages between 20 and 30 years; 5 female). We recorded CSP on the abductor pollicis brevis (APB) muscle after electrical stimulation of the index finger at 20 times the sensory threshold while subjects were performing mild muscle contraction. CSP recorded during following conditions: (i) baseline, (ii) teeth clenching (TC), (iii) dorsiflexion of the contralateral hand (DF), (iv) beginning of the reaction time (T1), v) middle of the reaction time (T2), and vi) end of the reaction time (T3). Tests were performed randomly except for the baseline condition. The onset and end latencies of total CSP, I1 phase, I2 phase, long-loop reflex (LLR) and post-CSP excitation period and suppression indices of I1 and I2 phases were measured. Each was compared with baseline recordings using Wilcoxon test.

Results: There was no statistically significant difference in latencies in any conditions compared to baseline. I2 suppression index increased during TC compared to baseline ( $48.8 \pm 28.1$ vs $27.0 \pm 20.9, \mathrm{p}=0.018)$. I2 suppression index was higher during $\mathrm{T} 1(37.4 \pm 30.8$ vs $27.0 \pm 20.9, \mathrm{p}=0.043)$ and during T3 $(44.7 \pm 30.6$ vs $27.0 \pm 20.9, \mathrm{p}=0.043)$ compared to baseline. Post-CSP excitation was also increased during TC (179.1 \pm 43.9 vs $111.4 \pm 53.0, \mathrm{p}=0.018$ ). During T3, post-CSP excitation was reduced compared to baseline, however, it was not statistically significant $(97.9 \pm 57.6$ vs $111.4 \pm 53.0, \mathrm{p}=0.068)$.

Conclusion: Contralateral extremity movement showed no effect on CSP. However, inhibition was decreased at the beginning and end of the reaction time. Post-CSP excitation was facilitated and inhibition decreased with TC. Facilitation of the H-reflex with TC was described in the literature and a similar supraspinal modulation is considered in absolute inhibition phase of CSP and post-CSP excitation period.

Keywords: cutaneous silent period, CSP, long loop reflex, LLR

\section{SS3-C03}

\section{Investigation of the effects of perinatal stress in rats on} synapse proteins in the enteric nervous system

Ece Alim $^{1}$, Füsun Erten ${ }^{2}$, Kazım Şahin ${ }^{3}$ İ́smail Nadir

Gülekon $^{4}$, Emel Ulupınar ${ }^{5}$, Ece Konaç ${ }^{6}$, Meltem Bahçelioğlu ${ }^{7}$

'Department of Anatomy, School of Medicine, Gazi University, Ankara, Turkey; Neuroscience and Neurotechnology Center of Excellence (NÖROM), Ankara, Turkey; ${ }^{2}$ Department of Veterinary Science, Pertek Sakine Genc Vocational School, Munzur University, Tunceli, Turkey; ${ }^{3}$ Department of Animal Nutrition, Faculty of Veterinary Science, Firat University, Elazığ, Turkey; ${ }^{4}$ Department of Anatomy, School of Medicine, Gazi University, Ankara, Turkey; ${ }^{5}$ Department of Anatomy, School of Medicine, Eskişehir Osmangazi University, Eskişehir, Turkey; ${ }^{6}$ Department of Medical Biology and Genetics, School of Medicine, Gazi University, Ankara, Turkey; 'Department of Anatomy, School of Medicine, Gazi University, Ankara, Turkey, Neuroscience and Neurotechnology Center of Excellence (NÖROM), Ankara, Turkey, Neuroscience Department, Institute of Health Sciences, Gazi University, Ankara, Turkey

Objective: The enteric nervous system is a system made up of neurons that form the "brain of the intestines" and function independently of the central nervous system. Neurexin is a presynaptic neuronal cell adhesion protein, and they bind with neuroligin, the postsynaptic protein. 'Stress' is defined as a condition that threatens body self-balance. The enteric nervous system provides regulatory control over a large number of gastrointestinal functions. Therefore, changes in the enteric nervous system function can lead to deep clinical symptoms in stress-related gastrointestinal disorders. In our study, we aimed to investigate the effects of perinatal stress on neuroligin- 1 and neurexin II $\alpha$ in the enteric nervous system of rats on different embryonic/neonatal days.

Methods: 36 female Wistar albino rats were mated. Embryos taken at embryonic days E16, E18, E20. and experimental animals born normally (up to postnatal 21 st day) were used. There are 8 groups in which prenatal and/or postnatal stress protocols were applied. Western blot analysis were performed to determine the protein levels of Neuroligin-1 and Neuroxin II $\alpha$ on the whole intestinal tissue.

Results: Statistically; prenatal stress on embryonic days E16, E18, E20 caused 20\%, 10\%, and 27\% decrease in Neuroligin1 protein levels in the collected embryonic intestinal tissues, and a $46 \%, 37 \%, 28 \%$ decrease in Neurexin II $\alpha$ protein levels, respectively. Postnatal or perinatal stress caused a decrease in Neuroligin-1 and Neurexin II $\alpha$ protein levels in all intestinal sections, but the highest reductions were observed in duodenal tissue in group 8 with Neuroligin-1 by $64 \%$ and in colon tissue in group 8 with Neurexin II $\alpha$ with $55 \%$.

Conclusion: General and regional decreases in protein levels in stress groups compared to control groups are remarkable. It is concluded that perinatal stress negatively affects the enteric nervous system by showing regional differences. The study was supported by Tübitak 3001 project numbered SBAG 118S031.

Keywords: perinatal stress, enteric nervous system, Neuroligin1 , Neurexin II $\alpha$, rat

\section{SS3-C04}

The effects of transcranial direct current stimulation in the treatment of motor functions after cerebral ischemia

\section{Güven Akcay ${ }^{1}$, Mutay Aslan², Narin Derin}

'Department of Biophysics, Faculty of Medicine, Akdeniz University, Antalya, Turkey; ${ }^{2}$ Department of Biochemistry, Faculty of Medicine, Akdeniz University, Antalya, Turkey

Objective: Cerebral ischemia occurs as a result of obstruction of some or all of the arteries feeding the brain, resulting in significant impairments in both motor and cognitive functions. In recent years, transcranial Direct Current Stimulation(tDCS), a noninvasive treatment method, are used frequently in the treatment of motor function disorders. In our study, it was aimed to investigate the effects of anodal and cathodal tDCS in the treatment of motor function after cerebral ischemia.

Methods: Twenty male Wistar rats weighing $300 \mathrm{~g}$ were divided into 4 groups as sham, ischemia/reperfusion(IR), IR+Anodal tDCS and IR+Cathodal tDCS. The IR model was 
created by middle cerebral artery 90 -minute occlusion. tDCS treatments were applied 30 minutes a day for 6 days after IR. Open Field (OF) test was used to evaluate motor functions. Glutamate and glutamine levels in the brain tissues were analyzed by mass spectrometry. Statistical analyzes were performed by One-Way ANOVA test. Statistical significance was accepted as $\mathrm{p}<0.05$.

Results: There was a statistically significant increase in glutamate and glutamine levels in both IR and IR+anodal tDCS groups compared to the sham group. In addition, in glutamate and glutamine levels was found to be higher anodal tDCS stimulation compared to the IR group. It was observed that there was a statistically significant decrease in glutamate and glutamine levels in IR+Cathodal tDCS group compared to IR group. In the $\mathrm{OF}$ test results in which motor function was evaluated, a significant increase was observed in the distance, velocity and frequency in IR+Cathodal tDCS group compared to IR group.

Conclusion: It was concluded that in the glutamate and glutamine levels of tDCS groups, anodal tDCS increased and cathodal tDCS decreased after cerebral ischemia. It has been concluded that cathodal tDCS can be used as a promising new method in the treatment of motor functions with glutamate inhibition in stroke treatment.

Keywords: glutamate, glutamine, anodal tDCS, cathodal tDCS

\section{SS3-C05}

Receptive fields of bursts and tonic spikes in the visual sector of thalamic reticular nucleus

Ulaş Mustafa Çiftçioğlu ${ }^{1}$, Friedrich Tobias Sommer ${ }^{2}$, Judith Ann Hirsch ${ }^{1}$

'Department of Biological Sciences, University of Southern California, Los Angeles, CA, USA; ${ }^{2}$ Redwood Center for Theoretical Neuroscience, University of California Berkeley, Berkeley, CA, USA

Objective: Like relay cells in the lateral geniculate nucleus of the thalamus (LGN), neurons in the thalamic reticular nucleus (TRN) fire in two modes, burst and tonic. Visual stimuli alter the firing mode of relay cells, as demonstrated by the receptive field's time course. Typically, the temporal field comprises a "triggering phase", that reflects neural preference for stimulus contrast and initiates spiking, and an earlier "priming phase" that reflects the non-preferred contrast. In LGN, the priming phase for burst receptive fields is stronger than for tonic. Here, we investigated if bursts in LGN and TRN are similar and, further, whether burst size depends on the visual stimulus.

Methods: We mapped receptive fields in TRN by computing the spike-triggered average (STA) of a Gaussian noise stimulus; $\mathrm{n}=88$ neurons from 13 anesthetized mice, and also analyzed a companion dataset from cat. To explore the relationship between the number of spikes in a burst and stimulus strength, we computed the inner product between the associated stimulus of a burst and the STA.
Results: Like LGN, the priming phase of burst receptive fields in TRN was stronger than tonic receptive fields (priming phase magnitude in mouse: burst $=36.4 \pm 15.1$, tonic $=22.3 \pm 15.0$; $\mathrm{p}=$ 0.027). And throughout the population, the number of spikes across bursts and visual stimulus strength were correlated (mouse: correlation $=0.052 \pm 0.079, \mathrm{p}=3 \times 10^{-6}$ ). Our findings were similar in cat.

Conclusion: Bursts in TRN coded different aspects of the visual environment than tonic spikes, like LGN. Further, the visual stimulus can alter the effect of a burst on postsynaptic targets through the number of spikes generated. Overall, our work highlighted characteristics of sensory processing by bursts in TRN that are conversed across species.

Keywords: TRN, LGN, inhibition, in vivo, thalamus, sensory

\section{SS3-C06}

Effects of cholinergic drugs on vibrotactile responses of neurons in the hindpaw representation of rat S1 cortex

Begüm Devlet Kılıçkap, Bige Vardar, Burak Güçlü

Institute of Biomedical Engineering, Boğaziçi University, Istanbul, Turkey

Objective: There is a lack of sufficient information regarding attentional mechanisms in S1 cortex modulated by cholinergic effects. The aim of this preliminary work is to study the vibrotactile responses of associated neurons by microinjection of cholinergic drugs.

Methods: We recorded spikes from 20 neurons, in the S1 hindpaw area of 6 ethics committee approved anesthetized rats, responding to vibrotactile stimuli on the skin (frequency: $5 \mathrm{~Hz}$; duration: $0.5 \mathrm{~s}$, amplitude: $50 \mu \mathrm{m}$ ). Before the vibrotactile stimuli, sham (aCSF), $100 \mu \mathrm{M}$ ACh, $100 \mu \mathrm{M}$ atropine (non-specific mAChR antagonist), $0.5 \mu \mathrm{M}$ MLA ( $\alpha 7 \mathrm{nAChR}$ antagonist) or $10 \mu \mathrm{M} \mathrm{DH} \beta \mathrm{E}(\alpha 4 \mathrm{nAChR}$ antagonist) were applied via microinjection. Average firing rates were calculated for three time periods: before stimulus (Rb), onset period (first $100 \mathrm{~ms}$ of stimulus) (Ro), and during the last $400 \mathrm{~ms}$ of vibrotactile stimulus $\left(\mathrm{Rd}^{*}\right)$. Repeated measures ANOVA was used to study the responsivity to vibrotactile stimuli.

Results: MLA and DH $\beta E$ applications had significant main effects on activity for the last 0.4 -s period of the stimulus $\left(\mathrm{Rd}^{*}\right.$ $\mathrm{Rb})(\mathrm{p}=0.003$ and 0.006 , respectively). These drugs decreased the average firing rates compared to the sham conditions for $\mathrm{Rd}^{*}-\mathrm{Rb}$. Additionally, MLA application significantly inreased the onset responsivity (Ro-Rb) to vibrotactile stimulus $(\mathrm{p}<0.001)$. The background activity $(\mathrm{Rb})$ was similar for all conditions.

Conclusion: Preliminary results suggest that nicotinic ACh receptor antagonists partially decrease the responsivity of S1 neurons to vibrotactile stimuli. This was observed especially after the onset period; and on the contrary, MLA increased the average firing rate during the onset. Our previous work on the electrical stimulation of BF showed that it is mostly synchronicity to the vibrotactile stimulus which is modulated by 
wide-spread cholinergic activation. The current study will be extended to measure the specific effects of nicotinic receptors in more detail. Support: Boğaziçi University Research Fund (no.17XP2)

Keywords: attention, cholinergic system, microinjection, somatosensation, vibrotactile

\section{SS3-C07}

P240 response to radial motion is correlated with autistic traits

Simge Aykan, Canan Kalaycioğlu

Department of Physiology, School of Medicine, Ankara University, Ankara, Turkey

Objective: Autistic traits are subthreshold deficits in healthy individuals similar to those present in autism spectrum disorders (ASD), such as social interaction and communication deficits, as well as restrictive/repetitive behaviors. Recent evidence has indicated that children with ASD show abnormal motion perception. In this study, the presence of these differences in healthy individuals with autistic traits was investigated by EEG recordings during motion stimuli.
Methods: Fifty-eight adult participants were recruited for the current study (25 females and 33 males, mean age $=23.38$ years, $\mathrm{SD}=3.35$ years). Autistic traits were assessed with Autism Spectrum Quotient [mean score $=17.59, \mathrm{SD}=4.80$, range (8; 27)]. Evoked potentials obtained by a radial expansion motion stimulus in low contrast $(10 \%)$ concentric circles with sinusoidal luminance modulation. The stimuli had 200 ms motion, which was followed by a $1000 \mathrm{~ms}$ interstimulus interval. Amplitudes and latencies of N160 and P240 components were evaluated at $\mathrm{Pz}$ and $\mathrm{CPz}$ leads respectively.

Results: There was no correlation between autistic traits and N160 component. P240 latency was positively correlated with autistic traits $[\mathrm{r}(56)=0.334, \mathrm{p}=0.010]$. No correlation observed for P240 amplitude.

Conclusion: Biological motion processing is known to be impaired in ASD subjects. Our stimulus might represent biological motion information, since the expansion evokes an illusion that something moves towards the subject. P240 has been shown to be affected by complexity of visual moving stimuli and more related to biological motion detection. As a result, our findings showing prolonged latency of P240 in individuals with high autistic traits are in concordance with literature.

Keywords: autistic traits, radial motion, visual evoked potential 


\section{Poster Presentations \\ (PP1 - PP10)}

PP1

Molecular and Cellular Neuroscience

\section{PP1-001}

Relationship of NLRP3 inflammasome in blood, IL-1beta and il-18 cytokine level with cognition in OCD

Betuil Önder ${ }^{1}$, Melike Tetik Oktay ${ }^{1}$, Neşe Direk ${ }^{2}$, Cansu Aykaç', Burcu Ekinci, Tutku Yaraşs, Aykut Kuruoğlu ${ }^{3}$, Çağatay Ermişs, Yavuz Oktay ${ }^{5}$, Tunç Alkın ${ }^{2}$

'Department of Neuroscience, Dokuz Eylül University, Izmir, Turkey; ${ }^{2}$ Department of Psychiatry, Dokuz Eylül University, Izmir, Turkey; ${ }^{3}$ Izmir International Biomedicine and Genome Institute, Dokuz Eylül University, Izmir, Turkey; ${ }^{4}$ Department of Child and Adolescent Psychiatry, Dokuz Eylül University, Izmir, Turkey; ${ }^{5}$ zzmir Biomedicine and Genome Center, Dokuz Eylül University, Izmir, Turkey

Objective: Research showed that inflammatory processes are included in many psychiatric disorders. NLRP3 Inflammasome which is formed by combination of NLRP3, ASC and Caspase-1 proteins and its activator NEK7 has a role in these processes. Relationship between neuropsychiatric diseases and NLRP3 has been previously detected, and its relationship with OCD has been shown for the first time by our research group. Findings of possible relationships between IL-1 $\beta$ and IL-18, which are the result of activation of NLRP3 inflammasome and neurocognitive impairment in OCD are limited. Cytokines may regulate cognition and neuronal-glial cell function for neurodegeneration or neuroregeneration. We aimed to investigate relationship between IL-1 $\beta$, IL-18 cytokine levels, NLRP3, ASC, Caspase1, NEK7 gene expression-protein levels, and cognition in OCD.

Methods: The study was performed at Dokuz Eylül University Hospital. 42 OCD patients aged between 18-45 were included. SCID-1, Y-BOCS, Hamilton Depression Scale were applied. Trail Making Test (TMT), Berg Card Sorting Test and Category Fluency Test were administered to evaluate cognitive functions. Schizophrenia, bipolar disorder etc. and inflammatory conditions were exluded. Cytokine levels were assessed by ELISA; NLRP3, Caspase-1, ASC, and NEK7 gene expressions were determined qPCR. Evaluation of the amount of protein encoded by the same genes was performed by Western Blotting.

Results: Weak negative correlations were found between IL-1 $\beta$ and TMT (B-A) ( $\mathrm{rs}=-36, \mathrm{p}=0.019)$; NEK7 protein level and TMT-B ( $\mathrm{rs}=-.3, \mathrm{p}=0.033)$. A weak negative correlation between TMT-B, TMT (B-A) and ASC protein levels was significant ( $\mathrm{rs}=-$ $.38, \mathrm{p}=0.03 ; \mathrm{r} s=-0.36, \mathrm{p}=0.04$, respectively). There was no relationship between other biological and cognitive variables $(\mathrm{p}>0.05)$.

Conclusion: IL-1 $\beta$, NEK7, and ASC levels may be associated with impaired cognition in OCD. Studies with larger samples about inflammatory processes are needed to develop new diagnosis/treatment methods.
The study funding was provided by The Scientific and Technological Research Council of Turkey (TÜBİTAK) grant \#217S128 (TA, YO).

Keywords: cognition, cytokine, NEK7, NLRP3, OCD

\section{PP1-002}

The hypotalamic glutamaterjic neurons modulate the effects of high fat diet on cognition

Hatice Kübra Ișıldar ${ }^{1}$, Rumeysa Atayolu², Pelin Dilsiz', Edanur Ateş Öz $z^{3}$, Muhammed İkbal Alp ${ }^{1}$

'Istanbul Medipol University, Institute for Health, Science and Technologies, Regenerative Restorative Medicine Research Center (REMER); ${ }^{2}$ Istanbul Medipol University Nutrition Dietetics, Istanbul, Turkey; ${ }^{3}$ Munich Technical University, Virology Institute, Munich, Germany

Objective: Certain groups of neurons in the arcuate nucleus in hypothalamus are responsible for regulate food intake. It is known that eating high-fat, causes disruptions in this signaling in the hypothalamus. Glutamate is the most common neurotransmitter in brain and plays a key role in LTP formation and learning. In this study, it is hypothesized that the negative effects of eating high-fat on cognition occur through hypothalamic glutamatergic neurons. The role of glutametergic neurons has been investigated in the effect of high-fat diet on food intake and memory in the hypothalamus for a long time.

Methods: In this study, $20 \mathrm{v}$-glut cre mice were used, and mice were injected into the hypothalamus, arcuate nucleus, intracranial virus (pAAV-EF1a-DIO-hM3D(Gq)-mCherry).A group of mice $(n=10)$ with a chronically high-fat diet, the other group $(\mathrm{n}=10)$ was fed with a standard pellet feed.Immediate stimulation of glutamatergic cells was achieved with chemogenetic stimulation method, chemogenetic eating and novel object test experiments were performed.At the end of the experiments, the brain sections of the mice were taken and microscope images were performed to determine virus injection.

Results: The findings of the study showed that glutamatergic neuron activation in the hypothalamus has a suppressive effect on eating in both groups. According to Novel object test data, it has been found that high fat diet for a long time has a negative effect on memory. Activation of the hypothalamus glutamatergic neurons has been found to help reduce this negative effect.

Conclusion: Eating high fat for a long time causes a memory impairment. This impairment shows a partial improvement with the activation of glutamatergic neurons in the hypothalamus. This result suggests that high-fat nutrition causes a desensitization in glutamatergic cells. It has also been found that activation of these cells directly suppresses eating.

Keywords: glutamate, high-fat diet, chemogenetics, hypothalamus, cognition 


\section{PP1-003}

The effect of Pea3 transcription factor on neural circuit formation

\section{Mustafa Doğukan Metiner ${ }^{1}$, Başak Kandemir ${ }^{2}$, Işıl Kurnaz ${ }^{1}$}

'Biotechnology Institute, Gebze Technical University, Kocaeli, Turkey; ${ }^{2}$ Department of Molecular Biology and Genetics, Faculty of Arts and Sciences, Başkent University, Ankara, Turkey

Objective: Pea3 proteins, a subgroup of the ETS family, consist of Pea3, Erm and Er81. These proteins are involved in the formation of motor neuron circuits in the nervous system, retinal differentiation, and axon elongation. The effects of the Pea3 protein on axon elongation mechanisms and how the phospho-mutant Pea3 affects axon elongation have been investigated in our laboratory. In this study, neural circuit formation was tried mimicked using cells expressing Pea 3 and Pea 3 phospho-mutants in a microfluidic system. The aim is to examine the axon elongation of neuronal cell models expressing Pea3 towards each other and to determine the phosphorylation motifs that directly affect this axon elongation.

Methods: For this purpose, the NSC-34 cell line was grown in DMEM High Glucose medium at $37^{\circ} \mathrm{C}$ and $5 \% \mathrm{CO} 2$ and transfection using lipofectamine. After cells which transfected with different Pea3 variants were seeded in opposite wells of cells transfected with Pea3-VP16 or mPea3 in the microfluidic system.

Results: In experiments with Pea3-VP16, axon elongation and migration were observed in Pea3-VP16 positive cells when mPea3 transfected or pCDNA3 transfected cells were seeded on the opposite side. When there were mPea3-S90A positive cells in the opposite well, less axon elongation was observed. In experiments with $\mathrm{mPea} 3$, it was observed that axon elongation was slightly less when mPea3-S90A transfected cells were seeded in the opposite well. When mPea3-S90E transfected cells were seeded on the opposite side, a significant decrease in axon elongation was observed. When mPea 3 transfected cells were seeded on both sides, it was observed axon elongation and migration.

Conclusion: According to our results, the selectivity of Pea3, which is involved in axon elongation, has been somewhat illuminated when constructing neural circuitry with different Pea3 phospho-mutant species. It was observed that the amount of axon elongation varied against different variants of Pea3.

Keywords: Pea3, axon elongation, neural circuit, microfluidic system

\section{PP1-005}

Oligodendrocyte and nerve fiber pathology in an experimental model of inflammation

Gunel Ayyubova, Leyla Yıldırım, İlaha Sadigi, Shahla Huseynova

Department of Histology, Embryology and Cytology, Azerbaijan Medical University, Baku, Azerbaijan

Objective: Clinical and experimental observations indicate that despite the advances in modern medicine, the inflamma- tion, being one of the major sources of reactive oxygen species, still plays a leading role in the initiation of brain damage and the development of diseases associated with nerve fiber pathology. It was demonstrated that inflammatory cytokines released by activated glial cells might destroy the tissue capability for spontaneous remyelination and contribute to disease progression. The purpose of this study was to describe the structural alterations in axons, dendrites and myelinated nerve fibers of the brain cortex at a model of neuroinflammation.

Methods: Systemic inflammation was induced in white rats by intravenous injection of purified lypolysacharide (LPS) from E. coli [Serotip 0111: B4] at a dose of $1.0 \mathrm{mg} / \mathrm{kg}$ dissolved in saline. Semithin brain sections were stained with toluidine blue and studied by light, ultrathin sections examined by Jeol 1400 electron microscope.

Results: Examination of brain sections revealed the increased number of reactive microglial cells and hypertrophic astrocytes throughout all cortical layers. Moreover, the electron microscopy revealed destructive changes in intracellular elements of cortical oligodendrocytes. Loss of integrity in cerebral capillaries, having been observed in brain sections, follows by the leakage of plasma components into the intercellular spaces and creates high osmotic pressure around the nerve fibers. Brain regions demonstrated noticeable myelin abnormalities such as myelin interruptions with extensive delamination in the form of diffuse separation of the sheath layers and myelin collapse. Dark myelin derivatives as the hallmark of demyelination were encountered among the neuropil elements. The myelin damage results in the degeneration of associated dendrites and axons.

Conclusion: Our study demonstrates that a persistent death of oligodendrocytes, being the most vulnerable brain cells, and damage to myelin should be considered as one of the key factors leading to the degeneration of accompanying axons at inflammatory conditions.

Keywords: neuroinflammation, brain cortex, oligodendrocyte, nerve fibers

\section{PP1-006}

Neuronal and glial cell loss does not occur in the aging zebrafish (danio rerio) brain

Narin Ilgım Ardıç ${ }^{1}$, Hande Özge Aydoğan ${ }^{1}$, Ayça Arslan Ergül ${ }^{2}$, Michelle M. Adams ${ }^{1,3}$

'Interdisciplinary Program in Neuroscience, Aysel Sabuncu Brain Research Center, Bilkent University, Ankara, Turkey; National Nanotechnology Research Center (UNAM) Bilkent University, Ankara, Turkey; Zebrafish Facility, Department of Molecular Biology and Genetics Bilkent University, Ankara, Turkey; National Magnetic Resonance Research Center (UMRAM), Bilkent University, Ankara, Turkey; ${ }^{2}$ Stem Cell Research And Application Center, Hacettepe University, Ankara, Turkey; ${ }^{3}$ Department of Psychology, Bilkent University, Ankara, Turkey

Objective: Previous work suggested that neuronal cell loss underlies cognitive decline but more recent studies in pri- 
mates, rodents, and even fruit-flies suggest that there is no significant cell loss in the aging brain. The current study was designed to determine whether the zebrafish brain, which shows a huge regenerative capacity, has significant cell loss during aging.

Methods: We analyzed the protein levels, distributions, and localizations of some key neuronal lineage and glial markers, during healthy aging of the zebrafish brain. For this purpose, immunohistochemistry and Western-blot techniques using antibodies directed against Hexaribonucleotide-BindingProtein-3, Microtubule-associated-protein-2, ELAV like neuron-specific RNA binding protein 3, Doublecortin-like-kinase1 , and Glial-fibrillary-acidic-protein were performed in the brains of young and old zebrafish.

Results: Initially, alterations in the number of neuronal and glial cells in the brain including two specific areas of high proliferation, pallium and optic tectum, were analyzed. Results indicated that in the aged brain there were no statistically significant differences in these markers. Secondly, to determine if there were changes in the protein levels of these markers independent of the cell number differences, Western-blot analysis was performed in whole brain lysates of a separate cohort of animals. The protein levels of the mature neuronal marker were stable across advanced age whereas the glial and immature neuron markers show age-related decreases. Interestingly, all of the previously mentioned protein markers showed differences between males and females.

Conclusion: The results overall support the finding that the number of neurons and glia do not change during brain aging in the zebrafish, which is consistent with other species. Moreover, protein level changes are not representative of neuronal and glial cell numbers. Finally, these data are more evidence that the zebrafish is an appropriate model for brain aging studies.

Keywords: aging, glia, neurogenesis, zebrafish

\section{PP1-008}

\section{Cathepsin Z expression in post-mortem cortex samples from Alzheimer's patients}

Gürol Atar ${ }^{1}$, Luke Martin², Athanasios Metaxas², Camilla Thygesen ${ }^{2}$, Bente Finsen ${ }^{2}$

'Interdisciplinary Neurosciences, Eskişehir Osmangazi University, Eskişehir, Turkey; ${ }^{2}$ Department of Molecular Medicine, University of Southern Denmark, Odense, Denmark

Objective: Alzheimer's disease (AD) is characterized by neuropathological findings such as brain atrophy,accumulation of amyloid plaques and neurofibrillary tangles, loss of neurons and synapses, and a chronic microglial reaction. Cathepsins are proteases expressed by all brain cell types. Microglia are known to express and secrete several different cathepsins that can support various immune functions. Cathepsin Z (CtsZ), a lysosomal cysteine protease, is highly expressed in cells of the immune system. This study examines the regional and cellular expression of Cts $Z$ in post-mortem frontal cortex tissue obtained from $\mathrm{AD}$ patients and non-demented controls.

Methods: Fresh-frozen post-mortem frontal cortex (BA 46) from female $\mathrm{AD}$ and control cases ( $\mathrm{n}=7-12$ /group) was used,obtained from the Netherlands Brain Bank. CtsZ was detected by IHC,and its co-localization to microglia/macrophages was evaluated by double IHC for CtsZ and Iba1. CtsZ immunoreactivity(IR) was scored on a scale from 0-4.CtsZ IR was digitalized and quantified using NIH ImageJ software, and CtsZ protein was quantified by ELISA.

Results: There was no difference in \% area covered by CtsZ IR in cortex between $\mathrm{AD}$ and non-demented cases. This accorded with ELISA data showing no between-group difference in CtsZ protein levels and findings of neuronal and glial CtsZ IR in most brains from both groups. Scoring of CtsZ in immunoreactive perivascular macrophages showed no differences between $\mathrm{AD}$ and non-demented cases. Interestingly, punctuated CtsZ IR, likely reflecting microglial-expressed CtsZ in association with amyloid plaques,occurred in much higher frequency in $\mathrm{AD}$ than non-demented cases according to the scoring process (MannWhitney, $\mathrm{p}<0.0001)$. This microglial expression of CtsZ was documented by co-localization of CtsZ to lysosomal-like bodies in activated Iba1 immunoreactive microglia in $\mathrm{AD}$ cases.

Conclusion: We found an increased CtsZ expression in microglia associated with amyloid plaques in cortex tissues of $\mathrm{AD}$ patients.CtsZ expression was observed not only in microglia,but also in neurons and perivascular macrophages. Detailed research is needed to elucidate the function of both neuronal- and microglia/macrophage-expressed CtsZ, including secreted CtsZ, in AD pathophysiology.

Keywords: Alzheimer's disease, cathepsin Z, cysteine proteases, microglia

\section{PP1-010}

\section{Cyto- and chemoarchitecture of the central cervical} nucleus

Esra Candar $^{1}$, Gülgün Şengül ${ }^{2}$

'Department of Neuroscience, Institute of Health Science, Ege University, Izmir, Turkey; 'Department of Anatomy, School of Medicine, Ege University, Izmir, Turkey

Objective: Central cervical nucleus $(\mathrm{CeCv})$ is a spinal cord gray matter nucleus located in upper spinal cord segments (C1C4) in human and other vertebrates, within the limits of lamina 7 , adjacent to lamina 10 as a continuous column. Animal studies have shown that $\mathrm{CeCv}$ neurons receive afferents from neck muscles, joints, semicircular canals, and project to the contralateral cerebellum, and ipsi- and contralateral vestibular nuclei. In this study, we aimed to reveal the cyto- and chemoarchitecture of $\mathrm{CeCv}$ for the first time in human spinal cord.

Methods: $10 \%$ formalin-fixed spinal cord was divided into segments and following cryoprotection $\mathrm{C} 1-\mathrm{C} 4$ segments were cut on a cryostat at a thickness of $35 \mu \mathrm{m}$. Nissl histochemical and calcitonin gene related peptide (CGRP), choline acetyltrans- 
ferase (ChAT), L-glutamic acid decarboxylase 65/67 (GAD 65/67), calbindin (Cb), calretinin (Cr) immunohistochemical stainings were performed, and sections were imaged under light microscopy and analyzed using ImageJ.

Results: $\mathrm{CeCv}$ neurons were multipolar, ovoid, triangular, fusiform in shape and average section area of the $\mathrm{CeCv}$ was measured as $286.11 \mathrm{~m}^{2}$. The largest $\mathrm{CeCv}$ neurons were $42.60 \times 16.65$ $\mu \mathrm{m}$ in size, and the smallest $11.84 \times 10.43 \mu \mathrm{m}$. In all C1-C4 segments, CGRP,ChAT,GAD 65/67, Cb, Cr immunoreactivities were observed in $\mathrm{CeCv}$ neurons. ChAT immunoreactivity was observed in very high, CGRP, GAD 65/67, Cb, Cr in high densities.

Conclusion: The cytoarchitecture of the $\mathrm{CeCv}$ was observed as similar to other species. For chemoarchitecture, CGRP, $\mathrm{ChAT}, \mathrm{Cb}, \mathrm{Cr}$ immunoreactivities have been shown in this study for the first time and interspecies variability was observed. GAD 65/67 was investigated in the human $\mathrm{CeCv}$ for the first time with this study and a high number of immunoreactivite neurons was found. We believe findings of this study will contribute to literature with detailed novel data for this important precerebellar nucleus.

This research was approved by Ege University Medical Research Ethics Committee (20-2.1T/32).

Keywords: central cervical nucleus, chemoarchitecture, cytoarchitecture, human spinal cord, immunohistochemistry

\section{PP1-011}

\section{Genetic contributors of schizophrenia: missense mutations of WFS1, HNF1B, FTL and MECP2 are involved in disease pathogenicity}

Hayriye Soytürk ${ }^{1}$, Burcu Biterge Süt ${ }^{2}$, Cansu Önal

'Department of Interdisciplinary Neurosciences, Bolu Abant izzet Baysal University, Bolu, Turkey; '2Department of Medical Biology, Niğde Ömer Halisdemir University, Niğde, Turkey; ${ }^{3}$ Department of Biology, Bolu Abant Izzet Baysal University, Bolu, Turkey

Objective: Schizophrenia is a chronic, complex genetic and severe mental illness affecting approximately $1 \%$ of the world's population. Schizophrenia is also a neurodevelopmental disorder involving a wide variety of social, cognitive, and perceptual functions. It is characterized by deterioration in personality, hallucinations and delusions, and cognitive impairments. The causes and mechanism of schizophrenia are still unknown, which may be related to its complexity and heterogeneity. Although there are several studies in the literature that are aimed at identifying genetic factors that contribute to schizophrenia and the hereditary patterns of its transmission, the genetic basis of schizophrenia has not yet been completely solved. Therefore, the aim of this study is to identify genetic factors linked with schizophrenia and to determine the molecular effects of identified mutations that may have a clinical significance.

Methods: Genetic variations associated with schizophrenia were retrieved from ClinVar public data repository. In silico analyses of pathogenicity, protein stability and structural alterations were performed using FATHMM and MUpro tools, and HOPE server.

Results: ClinVar analysis identified 659 genetic variations that are associated with schizophrenia. Majority of these genetic variations were pathogenic (23\%) in terms of clinical significance and exhibited germline transmission (92\%). $65 \%$ of all genetic variations identified were missense mutations. Specifically, missense substitutions in WFS1 (p.G780S), HNF1B (p.S148W), FTL (p.A96T), and MECP2 (p.A140V) were found to be damaging, resulted in decreased protein stability and introduced 3D structural changes.

Conclusion: Single nucleotide variations in WFS1, HNF1B, FTL, and MECP2 were identified as potential clinical risk factors in schizophrenia. We showed that the variations in WFS1, HNF1B, FTL, MECP2 resulted in reduced protein stability and structural alterations. In conclusion, the roles of these mutations in schizophrenia, as well as the relationship between them and their other diseases should be investigated in more detail.

Keywords: schizophrenia, genetics, missense mutation, pathogenicity

\section{PP1-014}

Creating a 3D neuronal-culture using alginate hydrogel optimal for neuronal survival

Bașak Dalbayrak ${ }^{1}$, Ekin Sönmez ${ }^{2}$, Habibe Kurt ${ }^{2}$, Müge İşleten Hoşoğlu $^{2}$, İsrafil Küçük ${ }^{3}$, Hale Saybaşıl1 ${ }^{1}$, Işıl Kurnaz ${ }^{2}$

'Institute of Biomedical Engineering, Boğaziçi University, Istanbul, Turkey; 'Institute of Biotechnology, Gebze Technical University, Kocaeli, Turkey; ${ }^{3}$ Institute of Nanotechnology, Gebze Technical University, Kocaeli, Turkey

Objective: Mimicking the cellular environment is important to observe cellular behavior. Hydrogels can be used as a 3Dimensional (3D) cell culture due to their properties like controllability. For this study, alginate which is extracted from brown algae is preferred because of their properties such as biocompatibility, nontoxicity, biodegradability. The guluronic acid residues of alginate are cross-linked with divalent cations to make hydrogel. Neurons do not have any integrins that recognize the alginate. The extracellular matrix (ECM) proteins can be added to alginate to increase neuronal growth. The aim of these experiments is to create an environment for neurons and optimize the alginate hydrogel.

Methods: The algae, Cytoseira barbata, are collected from Tuzla seaside, Istanbul. The extracted alginate is used for $3 \mathrm{D}$ cell culture. Characterization of the alginate is done before cellular experiments; Fourier-transform infrared spectroscopy (FTIR) and fluorescence spectroscopy. Mouse motor neuronlike cells (NSC-34) are used for cell encapsulation and cells are imaged every 24-hour under the light microscopy.

Results: After analyses, extracted alginates' $M / G$ ratio is found 0.6 , there is no fluorescence emission observed. All these results are compared with alginic acid, sodium salt (Sigma- 
Aldrich), and the results are similar to each other. For cellular experiments, the alginic acid, sodium salt, and extracted alginate are used. The alginate concentration is optimized $1 \%$ after trying concentrations of $0.5 \%, 1 \%, 2.2 \%, 2.5 \%$. After 72 nd hour the cells undergo necrosis, and there is observed cellular growth, barely.

Conclusion: Cellular growth is not an expected result, so the results are meaningful for these experiments. Alginate is a proper environment for neurons, but it needs some modifications. To extend the lifetime and cellular growth, the alginate modified with collagen are started but the experiments continues. These alginate and alginate-collagen platforms can be used for implantation after optimizations and the physical properties are improved.

Keywords: alginate, hydrogel, 3D neuronal-culture

\section{PP1-015}

Role of exosomes in malignant transformation of astrocytes

Ezgi Taşkan, Oğuz Kaan Kırbaş, Batuhan Turhan Bozkurt, Fikrettin Şahin, Burcu Kasapoğlu

Yeditepe University, Istanbul, Turkey

Objective: Glioblastoma multiforme (GBM) is the most common type of primary brain tumors with dismal prognosis. Glioblastoma tumors belong to the group of astrocytomas which originate purely from astrocytic cells in the brain parenchyma and usually do not affect neurons. The molecular mechanisms underlying the spread of these tumors to neighboring healthy tissue are unknown. The contribution of the exosomes to this process is not well understood. Exosomes are small nanovesicles released by all cell types and have specialized functions in a variety of key biological processes like intercellular genetic and metabolic communication as well as modulation of immune responses. Recently, their role in cancer and other pathological conditions has gained more attention. Their cargo has been studied in GBM and other cancer cells and they are considered as potential diagnostic biomarkers. However, their role in diffuse glioblastoma invasion has been overlooked. The aim of this study is to investigate the interactions of exosomes in malignant transformation of normal astrocytes and progression of GBM.

Methods: We treated normal human astrocyte (NHA) cells with exosomes harvested from commercially available GBM cell lines (U87-MG and A172). Changes in cell viability and proliferation, cell cycle, expression levels of cancer marker genes, TG2 protein level and activity were analyzed.

Results: We found that the treatment of GBM-derived exosomes resulted in an increase in cell viability of NHA cells upto $20-25 \%$ in $48 \mathrm{~h}$. Significant increases in cell proliferation, mRNA levels of GBM related marker genes like p53, Gli-1, Akt, EGFR, protein activity and level of TG2 were observed in NHA cells treated with GBM-derived exosomes.
Conclusion: The findings of our study indicated a link between the malignant transformation of normal astrocytes and their exposure to GBM-derived exosomes. We think that understanding the role of exosomes in this transformation will greatly contribute to the search of therapeutic options for this devastating cancer.

Keywords: glioblastoma multiforme, astrocyte, exosome

\section{PP2}

Neurodevelopment

\section{PP2-001}

Effect of maternal triphenyl phosphite exposure on rats from juvenile to adulthood in connexin43 expression on Cornu Ammon 3 Region

Petek Bilim ${ }^{1}$, Kübra Çelik², Gurur Garip ${ }^{3}$, Ahmet Giray

Yardımcı ${ }^{4}$, Buket Şura Pamuk ${ }^{4}$, Meral Baka ${ }^{3}$

'Department of Psychology, Faculty of Economics, Business Administration and Social Science, Toros University, Mersin, Turkey; ${ }^{2}$ Department of Neuroscience, Institute of Health Sciences, Ege University, Izmir, Turkey; ${ }^{3}$ Faculty of Medicine, Department of Histology and Embryology, Ege University, Izmir, Turkey; ${ }^{4}$ Faculty of Medicine, Ege University, Izmir, Turkey

Objective: Exposure to environmental chemicals during embryonic development increases the risk of autism in offspring by disrupting intercellular communication in the Cornu Ammon3 (CA3) region of the hippocampus, which has many synaptic pathways. Most research has focused on the effect of neurons and microglial prenatal inflammation in autism, but potential modifications of astrocytes and neuron-astrocyte communication have been less studied. In this context, the aim of the study is to investigate the effect of maternal exposure of triphenylphosphite (TPP), an environmental neurotoxic substance, on astrocyte junction channels in the hippocampal CA3 region of adolescent and adult rats with the expression of Connexin 43.

Methods: The study has 6 groups: Three group of juvenile rats: Control, VPA $(500 \mathrm{mg} / \mathrm{kg})$ and TPP $(100 \mathrm{mg} / \mathrm{kg})$ (Postnatal day=35) and the group of adult rats control, VPA $(500 \mathrm{mg} / \mathrm{kg}$ ) and TPP (Postnatal day=60). There are 3 pregnant rats in each group. Maternal SF, VPA, TPP ip was administered 12.5 days of pregnancy. Rats were perfused on P35 ( $n=10$ for each group) and P60 ( $n=10$ for each group) for connexin43 immunohistochemically staining. The immunohistological staining, the sections were incubated with anti-connexin43 antibody and the reaction was observed under a light microscope. The immunoreactivity data was obtained by using Image $\mathrm{J}$ and cellSens software.

Results: The Cornu Ammon 3 (CA3) area was considered. The results showed that connexin43 expression increased on CA3 region in VPA and TPP group (both juvenile and adult rats).

Conclusion: The increase of connexin 43 in the TPP group both in adolescence and adulthood proved impaired intercellular com- 
munication and increased neuroinflammation. Consequently, TPP exposure during embryonic development increases the risk of autism by disrupting synaptic transmission in the long term. However behavioral, biochemical and molecular analyzes are required to investigate the effect of maternal TPP exposure in multiple perspective.

Keywords: autism, connexin 43, neurodevelopment, triphenyl phosphite, valproic acid

\section{PP3}

Neuronal Excitability and Synapse Function

\section{PP3-001}

Electrophysiological investigation of the effects of high cholesterol nutrition on the brain

Hatice Keser, Selcen Aydın Abidin, İsmail Abidin

Department of Biophysics, Faculty of Medicine, Karadeniz Technical University, Trabzon, Turkey

Objective: Cholesterol, which is free and esters in the blood, is an important biological lipid found in membrane structures. In developed and developing countries, the rate of diseases such as cardiovascular disorders, metabolic diseases is increasing gradually related to high cholesterol nutrition. In addition, it creates a risk factor that may increase neuroinflammation and neurodegeneration in the central nervous system. Although cholesterol is one of the main components of the human brain, it is argued that dietary cholesterol metabolites 24-hydroxycholesterol and 27-hydroxycholesterol can cause cognitive disorders. The aim of this study is to investigate the changes that can be seen in the brain after feeding with a high cholesterol diet by electrophysiological methods.

Methods: Two study groups $(\mathrm{n}=6)$ were formed and 20-25 g male C57BL/6 race mice were given a high cholesterol diet ad libitium and the control group was given a standard mouse diet for 6 weeks. In vivo electrocorticography recordings were taken at the end of the diet protocol. The cerebral cortex of the anesthetized mice is exposed by craniotomy and the head is fixed with stereotaxic method, and $\mathrm{Ag} / \mathrm{AgCl}$ ball electrodes are placed in the cortex and recorded for 15 minutes. The records were analyzed with p-Clamp 10 software program, absolute power and total power calculations of brain waves were determined. Statistical comparisons were made by Mann Whitney U test.

Results: Although there was no statistically significant difference, a general decrease in absolute power and total power values of animals fed with high cholesterol was observed. Absolute power $\mathrm{p}$ values are delta $\mathrm{p}=0.73$, theta, alpha, beta $\mathrm{p}=0.82$, gamma $\mathrm{p}=0,94$ and total power $\mathrm{p}=0.94$.

Conclusion: Six weeks of high cholesterol nutrition has been shown to reduce brain activity. Our work on this subject continues.

Keywords: high cholesterol diet, electrocorticography, power analysis, mouse, cortex

\section{PP3-002}

The effects of sulfite exposure on ABR in the postnatal period

Narin Derin ${ }^{1}$, Güven Akçay ${ }^{1}$, Betül Danışman ${ }^{1}$, $\underline{\text { Kevser Zeynep Kiymaz }}^{2}$

'Department of Biophysics, Faculty of Medicine, Akdeniz University, Antalya, Turkey; ${ }^{2}$ Medicine Student, Alanya Alaaddin Keykubat University, School of Medicine, Antalya, Turkey

Objective: Sulfur compounds are among the most commonly used food additives, their anti-microbial and antioxidant effects. However, it has been suggested that sulfite has an audible brainstem response (ABR). Therefore, in this study, it was aimed to investigate the effects of sulfide exposure on ABR in the postpartum period.

Methods: Experimental section, two groups of control (20 Wistarr), postnatal (20 Wistarrats) were formed. While the control group was given to prenatal and postnatal periods; The postnatal group was given normal drinking water including prenatal and drinking water containing sodium metabisulphite at a dose of $100 \mathrm{mg} / \mathrm{kg} /$ day in the postnatal period. ABR recordings were married on the 60th day of the postpartum period at 8 and 16 $\mathrm{kHz}$. Student $\mathrm{T}$ Test was performed for statistical analysis.

Results: Some differences were found between the ABR wavelets of the control and postpartum group, but these differences were not statistically significant. In addition, II. At $8 \mathrm{kHz}$, the wave threshold is extended compared to the control.

Conclusion: As a result, it was determined that sulfite applied in the postnatal period has no significant effect on ABR records.

Keywords: ABR, sulfur, postnatal

\section{PP4}

Sensory and Motor Systems

\section{PP4-001}

Effect of expectation on perceptual processes: evidence from behavior and a mechanistic cortical model

Buse M. Urgen, Hüseyin Boyacı

Interdisciplinary Neuroscience Program, Aysel Sabuncu Brain Research Center \& National Magnetic Resonance Research Center (UMRAM), Bilkent University, Ankara, Turkey

Objective: Prior knowledge and expectations strongly shape perceptual decision-making mechanism. However, it is not clear whether and how they affect low-level sensory processes. In this study we investigated the effect of expectation on visual perception at the threshold level in a $2 \mathrm{AFC}$ paradigm.

Methods: Task-irrelevant cues, face or house, provided prior information about the likelihood of the upcoming target stimulus category. Participants' $(n=8)$ task was to report the spatial location of the target stimulus. We systematically manipulated the expectation's validity by setting the validity of the category-cue at 


\section{S132 18th Turkish Neuroscience Congress, 6-9 November 2020, Ankara, Turkey}

$100 \%, 75 \%, 50 \%$, and neutral in separate sessions. Duration thresholds (70.9\% success) were computed in neutral, expected and unexpected trials. Next, we used a mechanistic cortical model with dynamic priors to unravel the computational mechanism underlying the effects we found in the experiment. We implemented the model on a trial by trial basis. At each trial the prior did not remain fixed, but it was updated at each iteration.

Results: Our behavioral results showed that thresholds were higher in unexpected trials than in expected trials $[\mathrm{t}(7)=3.079$, $\mathrm{p}=0.018$ ]. However, thresholds of expected trials $(100 \%)$ were not different from neutral trials $[\mathrm{t}(7)=0.676, \mathrm{p}=0.521]$. Our modeling findings revealed that the observed increase of the thresholds in the unexpected trials is due to greater number of iterations that are required for a successful detection when the expectation and actual input disagree $[\mathrm{t}(7)=3.220, \mathrm{p}=0.015]$.

Conclusion: Our behavioral findings reveal that violation of expectations delay sensory processes. This happens because when expectations are violated further processing is required by the system to complete the sensory process. Our findings on mechanistic cortical model also demonstrate that a parsimonious cortical model can successfully explain human behavior.

Keywords: prior information, expectation, visual perception, cortical model

\section{PP4-002}

\section{The anatomy of the olfactory system}

Bahattin Paslı, Hasan Barış Ilgaz

Department of Anatomy, Faculty of Medicine, Hacettepe University, Ankara, Turkey

Objective: Olfactory pathways are related with the transmission of odor detection, food selection, reproductive, maternal behavior, saliva-gastric secretion, visceral and autonomic functions such as sensory-influence is associated with other behavioral responses that are related to the neurological system. The aim of this review is to reveal the anatomy, functions and clinical relations of the olfactory system.

Methods: Literature review was performed about the olfactory pathways. Also, 5 bovine brains were examined by the Klingler method.

Results: The upper face of superior nasal concha in the nasal cavity and the adjacent part of the nasal septum are related to the sense of smell, named as olfactory region. Central extensions of neurons in olfactory region form olfactory nerve. All of these extensions form fila olfactoria. Axons in fila olfactoria synapse with the second order neurons in olfactory bulb. The central extensions of the second order neurons form olfactory tract. From here lateral and medial olfactory striae arise. The fibers in lateral olfactory stria go to the part of the cerebral cortex. Most of the fibers in the medial olfactory stria begin from the anterior olfactory nucleus and pass through the anterior commissure to the contralateral olfactory bulb. Extensions of the second order neurons in olfactory bulb go to the piriform cortex, entorhinal cortex, anterior olfactory nucleus, locus caeruleus, medial septal nucleus, diagonal band of Broca, olfac-

tory tubercle and amygdaloid body. These regions are known as the olfactory cortex. The orbital part of frontal lobe is also related to the sense of smell. The connections of neurons in the entorhinal cortex to the hippocampus, and neurons in the olfactory bulb to the amygdaloid body allow the connection of olfactory pathways to the limbic system.

Conclusion: Knowing the anatomy, functions and clinical relations of the olfactory system are important for clinical evaluations and procedures.

Keywords: olfactory system, olfactory nerve, olfactory cortex, neuroanatomy

PP5

\section{Behavioral and Cognitive Neuroscience}

\section{PP5-001}

Is there any difference in recognizing facial expressions on self face and other's face? An ERP study

Arda Fidanc1, Esin Gürcan, Evrim Gülbetekin

Department of Psychology, Faculty of Medicine, Akdeniz University, Antalya, Turkey

Objective: Face recognition mechanism involves interpreting social and biological cues of others. Self-other distinction and understanding emotional facial expressions play a crucial role in social life. P300 is a known ERP component reflecting facial familiarity. However, it is not clear how humans recognize facial expressions on self-face and other faces. We aimed to investigate $\mathrm{P} 300$ component in processing facial expressions on participant's own, other's and morphed faces.

Methods: Sixteen students volunteered the study. Written informed consent form was obtained from each participant. Three stimulus type was used: self, other, morphed faces. Neutral, happy and sad facial expressions of five models were recorded while related videos were presented to elicit expressions. The same procedure was carried out for the participants. Facial expressions were morphed with self and models' faces. Stimuli were presented in random order by using E-Prime2.0. The participants were asked to observe faces. 32-channel EEG was used during the experiment. P300 component measured at electrodes P3, P4, CP1/CP5, CP2/CP6, FC1/FC5 and FC2/FC6.

Results: A four-way repeated measures ANOVA on P300 amplitude was conducted with expression(neutral,happy,sad), facial resemblance(self,other,morphed), hemisphere(left,right) and electrode site (P, CP, C, FC) as within subject factors. A significant main effect of Hemisphere was found $F(1.15)=11.210$ $\mathrm{p}<.01$. P300 was more positive at right hemisphere than left hemisphere. A significant main effect of Electrode, $\mathrm{F}(3.45)=26.038 \mathrm{p}<.001$ was observed, (larger amplitude at electrodes $\mathrm{P}, \mathrm{CP}$ than $\mathrm{C}, \mathrm{FC}$ ), bonferroni correction was applied for multiple comparisons. There was a significant interaction of Hemisphere and Facial resemblance $F(2.30)=3.292$ p $<.05$. P300 
effect at other's facial expressions in right hemisphere was more positive than it is in left hemisphere.

Conclusion: Right hemispheric dominance of P300 component seems to be associated with processing expressions of other faces but not own faces.

Keywords: facial expressions, facial familiarity, face recognition, p300

\section{PP5-002}

Are the effects of chronic stress on memory different in female and male mice?

Aslıhan Bahadır Varol ${ }^{1}$, Emre Cem Esen ${ }^{2}$, Gül Yalçın Çakmaklı ${ }^{3}$, Hülya Karataş Kurşun ${ }^{1}$, Müge Yemişçi Özkan ${ }^{1}$, Turgay Dalkara ${ }^{1}$, Bülent Elibol ${ }^{3}$, Emine Eren Koçak ${ }^{1,4}$

'The Institute of Neurological Sciences and Psychiatry, Hacettepe University, Ankara, Turkey; '2Department of Psychiatry, Faculty of Medicine, Balıkesir University, Balıkesir, Turkey; ${ }^{3}$ Department of Neurology, Faculty of Medicine, Hacettepe University, Ankara, Turkey; ${ }^{4}$ Department of Psychiatry, Faculty of Medicine, Hacettepe University, Ankara, Turkey

Objective: It is well known that depressive patients have memory dysfunction. Recent studies showed that there may be differences between males and females in the neurobiological mechanisms and molecules involved in major depression. We aimed to study whether chronic stress affects short- and longterm memory functions differently depending on the sex.

Methods: We exposed C57BL/6 female (control $\mathrm{n}=10$, study $\mathrm{n}=22$ ) and male (control $\mathrm{n}=6$, study $\mathrm{n}=16$ ) mice to predator, restraint and tail suspension stresses alternately for 35 days. To examine short- and long-term memory, novel object recognition test was performed 1 and 24 hour after the familiarization session. Univariate analysis of variance was used for the statistical analysis.

Results: There was a significant difference between female and male mice in short-term memory performance $[\mathrm{F}(3.54)=6.259$ $\mathrm{p}=0.001]$, but not in long-term memory performance $[\mathrm{F}(3.51)=$ $1.008 \mathrm{p}=0.321]$.

Stress negatively affected both short- and long-term memory performances $[\mathrm{F}(1.54)=237,549 \mathrm{p}=0.008 ; \quad \mathrm{F}(1.51)=23.044$ $\mathrm{p}=0.00000001$, respectively]. There was a significant stress $\mathrm{X}$ sex interaction on both short- and long-term memory $[\mathrm{F}(1.54)=12.297 \mathrm{p}=0.007 ; \mathrm{F}(1.51)=4.652 \mathrm{p}=0.036$ respectively $]$.

Conclusion: While stress affected both short- and long-term memory performances of male mice, it affected only long term memory performance of females. Negative impact of chronic stress on short- and long-term memory performances was more evident in male than female mice. Our results indicate that stress may affect memory functions of male and female mice through different neurobiological mechanisms, which should be investigated by future studies.

This project was supported by TÜBİTAK SBAG 118 S558.

Keywords: memory, chronic stress, gender differences

\section{PP5-003}

The involvement of attention and masking in controlling stimulus visibility

Afife Konyalı $^{1}$, Hulusi Kafalıgönül ${ }^{2}$

'Interdisciplinary Neuroscience Program, Bilkent University, Ankara, Turkey; ${ }^{2}$ National Magnetic Resonance Research Center (UMRAM), Bilkent University, Ankara, Turkey

Objective: Information is processed at different stages of the visual system. An important question in cognitive neuroscience is how attention modulates these distinct processes and hence affects the recognition of visual objects. Visual masking paradigms provide an important framework to shed light on this basic question. In particular, different processes have been proposed to be involved in paracontrast masking. For instance, brief and prolonged inhibitions have been observed at short and long stimulus onset asynchronies (SOAs), respectively. They have been considered to reflect low- and high-level inhibitory mechanisms in the parvo-dominated object recognition pathway.

Methods: In the present study, using a paracontrast masking paradigm, we aimed to understand how spatial attention affects these inhibitory mechanisms. As in previous studies (e.g., Agaoglu et al., 2018), we manipulated spatial attention by changing set-size in the visual field and employed a contour identification task on the visual target under different SOA conditions.

Results: Two-way repeated measures ANOVA on the behavioral performance values revealed a significant main effect of SOA $(\mathrm{p}<.001)$ and a two-way interaction between SOA and setsize $(\mathrm{p}=.007)$. To understand the nature of two-way interaction, we performed additional follow-up tests. We found a simple effect of set-size at both short $(\mathrm{p}=.043)$ and long $(\mathrm{p}=.044)$ SOA values over which brief and prolonged inhibitions were observed, respectively. Interestingly, these attentional modulations were distinct such that an increase in set size decreased brief inhibition while increasing prolonged inhibition.

Conclusion: Overall, these results indicate that attention can take place at both low- and high-level visual processing. More importantly, they further suggest that the effects of attention were differential on these processes which were involved in controlling stimulus visibility.

Support: This work was supported by The Scientific and Technological Research Council of Turkey (TUBITAK Project Number 119K368).

Keywords: attention, masking, inhibition, temporal dynamics, visibility

\section{PP5-005}

Open source software use in rodent behavioral testing for markerless tracking and multi-dimensional analysis

Safa Andaç ${ }^{1}$, Cemal Akmeşe ${ }^{2}$, Güneş Ünal ${ }^{2}$

${ }^{1}$ Boğaziçi University Cognitive Science Program, Istanbul, Turkey; ${ }^{2}$ Department of Psychology, Behavioral Neuroscience Laboratory, Bogaziçi University Istanbul, Turkey 
Objective: Animal tracking software constitute the basic data collection and analysis method for behavioral neuroscience research utilizing rodent models. Minimizing subjective assessment of specific behaviors during animal testing is critical for the validity and replicability of the acquired data. However, classical animal tracking software are offered with limited update options, failing to produce the flexibility required by behavioral testing. This work is an open source program modification and application project to be utilized in several behavioral paradigms.

Methods: First, we added new modules for online recording and manipulation of behavioral tests to the DeepLabCut, an open source software utilizing deep learning algorithms, and created a new interface. Second, we tested Wistar rats for general locomotor activity (open field test), behavioral despair (forced swim test), anxiety (elevated plus maze), working memory (Y-maze) and spatial navigation (Morris Water Maze), and analyzed the acquired data in the aforementioned system.

Results: We acquired reliable animal tracking and behavioral classification in all the utilized behavioral tasks without using a physical marker on the animal. We managed to produce consistent classification of specific behaviors that could not be reliably identified in different tasks. We found that the virtual tags we used to reveal the relationship between specific extremities led to similar results in other behavioral tasks.

Conclusion: The system we have modified for the basic needs of laboratories using rodent models and equipped with a new interface, provided us with a level of behavioral selectivity that could not be achieved before. Tested in several cognitive and affective tasks, it is capable of encoding complicated paradigms that contain more than one animal or other moving objects. As the experimental setup can be controlled online based on the behavior of the animal, this animal tracking program can be used in sync with closed-circuit electrophysiological recordings.

Keywords: behavioral neuroscience, deep learning, neural network, animal tracking, behavioral data analysis

\section{PP5-006}

Separation of active sensing movements from taskrelated movements via machine learning approaches

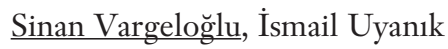

Department of Electrical and Electronics Engineering, Hacettepe University, Ankara, Turkey

Objective: Animals routinely exhibit active sensing movements during their behavioral tasks to enhance the sensory feedback perceived from the environment. Understanding the behavioral mechanisms of this phenomenon necessitates separating the active sensing movements from the task-related movements. This requires hand labeling the data. In this study, we use machine learning to automatically separate active sensing movements from task-related movements. Besides, we plan to further classify the types of active sensing movements adopted by the animals.
Methods: To achieve our objectives, we use a dataset about the refuge tracking response of Eigenmannia virescens. Dataset consists of 710 experimental trials collected under various sensory saliency conditions. Each trial includes fish's response to the movement of a sinusoidally moving PVC refuge in a single linear dimension. The movements of the fish, in response to refuge movements, are divided into epochs, separated by the zero crossings of the fish velocity. Then, we used the 'Bagged Trees' algorithm to train a classifier that separates the active sensing movements from the task-related movements of the fish.

Results: We used 5-fold cross-validation to assess the classification performance of our model. Our results indicate that Bagged trees can detect active sensing movements with 91\% accuracy on the test data. Besides, we classified the active sensing epochs into various stereotyped movements, such as scanning, drift, reset and wiggle. Our classifier can also separate the active sensing movements into these classes with $89 \%$ accuracy.

Conclusion: Despite promising classification results with $91 \%$ accuracy, there is still an important gap toward automatic clustering of behavioral movements. In the future, we plan to generate state transition diagrams of the fish that quantify transition probabilities from one type of movement to the others. We will use this probabilistic information to boost the classification results by modulating the scores assigned to different classes.

Keywords: active sensing, behavioural classification, computational neurosience, machine learning

\section{PP5-007}

Does similarity between species matter? Holistic face processing for great ape faces

Muhammed Nurullah Er, Enes Altun, Evrim Gülbetekin Department of Psychology, Akdeniz University, Antalya, Turkey

Objective: Holistic face processing is one of the major human cognitive mechanisms. It may be defined as perceiving individual features of face together into one whole context. Although humans process human faces holistically it's not clear whether holistic face processing is valid for faces of other species. The study aimed to investigate the holistic face processing in human participants for all great ape species (bonobos, chimpanzees, gorillas, orangutans, and humans) by using the part-whole task.

Methods: Two studies were conducted. Twenty undergraduate students participated in Study-1. Forty great ape faces were used as stimuli. In the first stage of the study, we presented stimuli with a $2 \mathrm{sec}$ interstimulus interval(ISI) in random order. The participants were asked to discriminate whether the presented stimulus was a human or non-human face. In the second stage, we tested if the subjects could recognize the faces in the first stage. Galvanic skin response(GSR) was measured. Seventy-five undergraduate students participated in Study-2. 
Part-Whole task was conducted to examine holistic face processing for each species. There were 4 target faces for each species, and a foil face for each target face. There were 12 trials for the whole and 12 trials for part condition. All stimuli were presented in random order. Two isolated face parts were presented in part condition while 2 whole faces were presented in whole condition. In both tasks, one single feature of these faces differed from the target face. Participants had to make choice between images.

Results: GSR were analyzed with Brainvision Analyzer and repeated measures ANOVA was conducted. Results indicated that there was no significant difference in GSR for different species ( $p>.05)$. According to pairwise t-tests, holistic face processing was observed for only human faces $t(74)=-4.385$, $\mathrm{p}=.001)$.

Conclusion: Our findings have shown that holistic face processing mechanism is observed only for our own species.

Keywords: face processing, holistic, part-whole, species-specific effect

\section{PP5-008}

Psychotic and autistic features in the general population are positively related to each other

$\underline{\text { Oğuzhan Bayram }}^{1}$, İrem Saçkesen ${ }^{1}$, Ömer Faruk Albayrak ${ }^{2}$, Çă̆ıl İnal ${ }^{3}$, Erhan Nalçacr ${ }^{3}$, Simge Aykan ${ }^{3}$

${ }^{1}$ Faculty of Nursing, Ankara University, Ankara, Turkey; ${ }^{2}$ Department of Psychology Faculty of Languages History and Geography, Ankara University, Ankara, Turkey; ${ }^{3}$ Department of Physiology, Faculty of Medicine, Ankara University, Ankara, Turkey

Objective: Neurodevelopmental diseases have common neurological foundations although they are categorized as independent entities. In addition, they are also seen in the general population at sub-diagnostic levels (e.g., psychotic and autistic traits). The aim of the currentstudy is to investigate the relationship between psychotic and autistic traits in general population.

Methods: Healthy participants, that are at least university students [132 women, 105 men; age=19.51 SD=1.32, range (18; 23)] were included in the study. The Magical Ideation Scale (MIS), which assesses psychosis-like experiences, and the Autism Spectrum Quotient (AQ), which measures autistic traits were completed by the participants. Increased scores on both scales indicate an increased symptom burden.

Results: In the total group, a positive correlation was found between MIS and AQ total scores [ $\mathrm{r}(235)=0.153, \mathrm{p}=0.018]$, also between attention to detail subscale and MIS $[\mathrm{r}(235)=0.189$, $\mathrm{p}=0.003]$. While the relationship between MIS and attention to detail was preserved in female participants $[\mathrm{r}(130)=0.216$, $\mathrm{p}=0.013]$, the relationship between MIS and AQ was preserved in male participants $[\mathrm{r}(103)=0.228, \mathrm{p}=0.020]$. In addition, a relationship was found between the sub-score of communication and MIS in male participants [ $\mathrm{r}(103)=0.243, \mathrm{p}=0.013]$. It was concluded that the relationship between MIS and AQ observed in the total group resulted from male participants, and the attention to detail with MIS resulted from female participants.

Conclusion: In this study, psychotic and autistic traits have been shown to increase in relation to each other. This finding is compatible with both traits having neurodevelopmental origins. The observed gender differences are in line with the literature. Rosen et al. (2019) shown that focus and attention disorders are high in women who are at high risk for psychosis. In addition, in neuroimaging studies that examine the relationships between gender and autism, it is suggested that the brain characteristics of autism are not only quantitatively but qualitatively differ between genders.

Keywords: autism spectrum quotient, autistic traits, magical ideation scale, psychotic traits, gender differences

\section{PP5-009}

\section{Assessment of spontaneous activity in MPTP-induced Parkinson models: standard indexes}

Deniz Önal, Özbeyen Atalay, Bilge Pehlivanoğlu

Department of Physiology, Faculty of Medicine, Hacettepe University, Ankara, Turkey

Objective: Parkinson's disease (PD), proceeds with the degeneration of dopaminergic neurons in the central nervous system, results in a serious and life-challenging clinical picture affecting the mechanisms related to voluntary movement such as bradykinesia, rigidity, postural imbalance and resting tremor. The importance of the experimental studies on $\mathrm{PH}$ is increasing, since it is a progressive neurodegenerative disease with gradually worsening symptoms and treatment mainly limited to symptomatic drugs or approaches, Systemic administration of 1-methyl-4-phenyl-1,2,3,6-tetrahydropyridine (MPTP) is one of the most widely used methods as PD model in laboratory animals. The model employed and therapautic and/or prophylactic approaches tested are evaluated by behavioral test batteries including locomotor and spontaneous activity tests, besides histochemical examinations.

Methods: As, spontaneous activity tests are evaluated using many different parameters in the literature; we aimed to examine new indices that will enable a more objective and precise assesment of spontaneous activity data in the mouse PH model. An animal model of acute PD was developed by administering a single dose of MPTP (16 mg/kg; ip) to adult male C57BL/6 mice $(n=29)$. The development of PD was confirmed before and after drug administration using locomotor (forced walking test on a horizontal bar) and spontaneous activity (spontaneous activity in a cylinder) tests.

Results: The most used parameters in the literature to evaluate spontaneous activity are hindlimb step number $(\mathrm{H})$, forelimb-hindlimb step ratio $(\mathrm{F} / \mathrm{H})$, grooming time $(\mathrm{G})$, rearing (R). For the standardization of these variables, activity index $(\mathrm{F}+\mathrm{H} / 180-\mathrm{G})$, forelimb index $(\mathrm{F} / \mathrm{F}+\mathrm{H})$, hindlimb index 


\section{S136 18th Turkish Neuroscience Congress, 6-9 November 2020, Ankara, Turkey}

$(\mathrm{F}+\mathrm{R} / 180-\mathrm{G})$ were calculated and they were found to be more sensitive than other parameters that are widely used for PD diagnosisi and prgression in mice.

Conclusion: In conclusion, using the indexes suggested for interpretation of spontaneous activity tests, as in locomotor activity, may allow comparison of the results of different study groups and more subjective evaluations.

Keywords: Parkinson's disease, MPTP, spontaneous activity, behavioral tests

\section{PP5-010}

Neural correlates of color discrimination: relation to linguistic categories

Seyma Koç Y1lmaz ${ }^{1}$, Didem Gökçay ${ }^{2}$, Emre Özgen ${ }^{3}$

${ }^{\prime}$ Department of Cognitive Science, Informatics Institute, Middle East Technical University, Ankara, Turkey; '2Department of Health Informatics, Informatics Institute, Middle East Technical University, Ankara, Turkey; ${ }^{3}$ Department of Psychology, Yaşar University, Izmir, Turkey

Objective: Is color perceived categorically? This is a major question in terms of testing the Sapir-Whorf hypothesis in the perception domain. Specifically, this hypothesis would predict that discriminating colors that are lexically classified into different categories, such as "green" and "blue" (cross-category), is easier than discriminating colors that belong to the same lexical category, such as two shades of green (within-category). This pattern is referred as categorical color perception and has been supported by a considerable number of studies. An essential question in this line of research is whether within-category and cross-category color pairs are separated by equal distances.

Methods: In the present fMRI experiment, we described three color pairs on the green-blue spectrum with equal hue differences, constant saturation and lightness using the CIE LCH color model. The color stimuli were manipulated according to linguistic relations, forming a within-blue pair, a within-green pair and a cross-category pair. Participants $(n=20)$ completed a two-alternative forced-choice color discrimination task.

Results: Our behavioral results revealed a pattern inconsistent with typical categorical effect. Rather than demonstrating a perceptual advantage for discriminating colors at the greenblue boundary, performance linearly declined throughout the selected region $(\mathrm{ps}<0.001)$. Comparisons of brain activations across categorical conditions revealed differences in task-difficulty, attention, and memory-related neural circuits (ps<0.01).

Conclusion: When the neuroimaging results were compared with behavioral findings, the activations related to color discrimination suggest the involvement of high-level, predominantly performance-related processes during color discrimination rather than linguistic interference.

Support: The authors are grateful to Prof. Dr. Ergin Atalar for access to the MRI facility at National Magnetic Resonance
Research Center (UMRAM), Bilkent University. The first author has been financially supported by TÜBİTAK (BİDEB 2211-E).

Keywords: color discrimination, categorical color perception, color spaces, fMRI

\section{PP5-011}

Exposure-based perceptual learning in aging zebrafish

$\underline{\text { Ayșenur Karaduman }^{1}}$, Elif T. Karoğlu Eravşar², Michelle M. Adams ${ }^{3}$, Hulusi Kafalıönü̈l ${ }^{4}$

'Interdisciplinary Neuroscience Program, Aysel Sabuncu Brain Research Center, Bilkent University, Ankara, Turkey; ${ }^{2 N a t i o n a l ~ N a n o t e c h n o l o g y ~}$ Research Center (UNAM), Bilkent University, Ankara, Turkey; ${ }^{3}$ Department of Psychology, Bilkent University, Ankara, Turkey; ${ }^{4}$ National Magnetic Resonance Research Center (UMRAM), Bilkent University, Ankara, Turkey

Objective: Repeated practice of a discrimination task gradually improves the ability to differentiate the direction of visual motion through perceptual learning. It has been considered as a promising approach for improving motion perception of older individuals who have difficulties in this important aspect of vision (Bower \& Andersen, 2012). We examined the effects of repeated exposure to the visual motion on the direction discrimination performance of 20 young (7-10 months) and 19 old (24-40 months) zebrafish. We used both wild-type (AB-strain) and mutants with attenuated age-related cognitive decline due to decreased levels of acetylcholinesterase (achesb55/+).

Methods: The zebrafish were exposed to repeated rightward and leftward drifting sine $\neg$ wave gratings with $45 \%$ contrast and $0.1 \mathrm{c} / \mathrm{deg}$ spatial frequency for 5 consecutive days. The normalized optomotor responses (OMR) to the gratings in each day were computed.

Results: One-sample t-tests comparing OMR to the baseline level showed that adult zebrafish exhibited significant $(\mathrm{ps}<0.01)$ negative OMR (position shift in the opposite direction of visual motion) in each experimental day. However, the results of linear mixed-effects model showed that number of exposure days to the motion (i.e., learning effect), genotype, and age of zebrafish did not have a significant effect on negative OMR (ps $>0.05$ ). The model also did not report any significant interaction between these factors (ps>0.05).

Conclusion: Our findings suggest that visual motion elicits significant negative OMR in adult zebrafish. Not having a robust effect of exposure-based perceptual learning might suggest that OMR is a product of sensory processing which mostly depends on low-level physical characteristics of visual motion rather than more complex computations involving learning.

Support: This work was supported by The Scientific and Technological Research Council of Turkey (TUBITAK Project Number: 219S133).

Keywords: perceptual learning, motion perception, aging, zebrafish, cholinergic system 
PP5-012

Investigation of six months continuous positive airway pressure treatment efficacy on severe OSAS patients using event related oscillations and neuropsychological tests

Deniz Yerlikaya ${ }^{1}$, Nurhak Demir ${ }^{1}$, İbrahim Öztura ${ }^{2}$, Görsev G. Yener ${ }^{3}$

'Department of Neurosciences, Institute of Health Sciences, Dokuz Eylül University, Izmir, Turkey; ${ }^{2}$ Department of Neurology, Dokuz Eylül University Medical School, Izmir, Turkey; ${ }^{3} / z m i r$ Biomedicine and Genome Center, Dokuz Eylül University Health Campus, Izmir, Turkey

Objective: Obstructive Sleep Apnea Syndrome (OSAS) is a sleep disorder which might cause cognitive impairments in time due to apneas/hypopneas occurring during sleep. Studies showed that Continuous Positive Airway Pressure (CPAP) treatment can be effective on cognitive impairments. Event Related Oscillations (EROs) are used as a sensitive measure for investigating cognitive processes. Delta oscillatory responses are known to reflect especially the attention and working memory processes. Impairments of these processes are commonly reported in OSAS. In this study, we aimed to investigate the efficacy of six-months CPAP treatment using EROs.

Methods: 25 male severe OSAS patients were included to the study. EEG recordings were recorded and detailed neuropsychological tests were applied at baseline and after six-months of CPAP. Visual oddball paradigm was used in the experiments. Eye movements were corrected with ICA. Artifacts were automatically removed. Delta oscillatory responses $(0.5-3.5 \mathrm{~Hz})$ were obtained by applying selective filters and peak-to-peak amplitudes were measured in a time-window of $0-800 \mathrm{~ms}$. In the statistical analyses, Repeated measures of ANOVA and paired sample t-test was used.

Results: ANOVA did not reveal a significant main TREATMENT effect on delta oscillatory responses $[\mathrm{F}(1.24)=4.053$, $\mathrm{p}=0.055)]$. Beck Depression Inventory scores $(\mathrm{p}=0.02)$ and Stroop Test resistance to interference scores $(p=0.019)$ were reduced significantly after CPAP.

Conclusion: This is the first study in the literature that investigates CPAP efficacy by using EROs. After six-months of CPAP treatment no differences were found on delta oscillatory responses compared to baseline. Although the OSAS patients' baseline neuropsychological test scores were within age and education norms, improvement was observed in depression and inhibition scores after treatment. These findings point out that the treatment might be effective on cognitive processes. Future studies should investigate theta, alpha and beta oscillatory responses and include a healthy control group in order to arrive at a better understanding of these results.

Keywords: event related oscillations, neuropsychological tests, OSAS

\section{PP5-013}

Effects of cognitive styles on activator and inhibitory processes in the brain

Dilem Dinç ${ }^{1}$, Tolgay Ergenoğlu ${ }^{2}$, Sudem Nur Turan ${ }^{1}$, Sabri Ferikoğlu ${ }^{1}$, Yahya Şahbudak ${ }^{3}$

'Department of Psychology, Mersin University, Mersin, Turkey; ${ }^{2}$ Department of Physiology, Mersin University, Mersin, Turkey; ${ }^{3}$ Department of Psychology, Çağ University, Mersin, Turkey

Objective: During the individuals' cognitive process, some stimuli must be suppressed, while some activated. Recent research have tended to evaluate analytic-holistic styles as personality traits and indicated that the information processing processes in those prone to one of these styles are different from each other. Our study aims to investigate the activator and inhibitory processes in the brain for individuals prone to these cognitive styles.

Methods: Cognitive Style Analysis (CSA) test was used to determine cognitive style tendencies. ERP records were taken from 30 electrodes with the Go-NoGo paradigm. According to CSA test, 29 volunteers $(21 \pm 2.49$ years old) with 15 AnalyticWholistic cognitive style tendency participated in our study. $\mathrm{N} 2$ and P3 potential amplitude and latencies of the data obtained to determine the differences between cognitive style groups were evaluated by repeated variance analysis (ANOVA). In addition, the relationship between Go task reaction times and reaction times obtained during CSA task was evaluated by pearson correlation analysis.

Results: The main effect of cognitive styles on Go-P3 amplitude tends to be close to significance $(\mathrm{p}=.067)$. The main effect of cognitive styles on NoGo-P3 amplitude is significant $(p=.022)$. There was not a significant difference in the reaction times of Go task for the reaction times of cognitive styles $(\mathrm{p}=.161)$. A positive correlation was observed between Go task and reaction time during analytic cognitive style task $(\mathrm{r}=.39)$.

Conclusion: Go-P3 and NoGo-P3 amplitude values were higher in the group with a tendency for holistic cognitive style. It was observed in the NoGo-P3 appeared later in the leftfrontal in the analytic cognitive style tendency group. Those who spend a lot of time in Go task also spend a lot of time in analytic cognitive style task. These findings suggest that individuals prone to holistic cognitive style spend more time on tasks and direct their attention more.

Keywords: analytic cognitive style, event-related potentials, Go-P3, NoGo-P3, wholistic cognitive style.

\section{PP5-014}

Impairment in saccadic eye movements in Parkinson's disease

Koray Koçoğlu ${ }^{1}$, Müge Akkoyun ${ }^{1}$, Berril Dönmez Çolakoğlu², Raif Çakmur ${ }^{2}$, Gülden Akdal ${ }^{2}$

'Department of Neurosciences, Institute of Health Sciences, Dokuz Eylül University, Izmir, Turkey; ${ }^{2}$ Department of Neurology, Faculty of Medicine, Dokuz Eylül University, Izmir, Turkey 
Objective: Eye movement recordings display changes in the oculomotor pathway in which some conditions have been linked to Parkinson's disease (PD). Moreover, previous studies have revealed conflicting results for saccade latency either shorter or longer. The aim of this study was to investigate prosaccades responses in early staged PD compared to healthy controls (HCs).

Methods: Eye movement recordings were administered to 17 patients with $\mathrm{PD}$ (age: $64.2 \pm 9.6$, disease duration $4.5 \pm 5.7$ years)

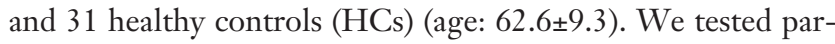
ticipants to probed their performance [saccadic reaction time (SRT), anticipatory and corrected saccade responses] on prosaccades trials. Motor disability of PD was assessed using the UPDRS-III (18.6 \pm 10.5$)$. Hoehn \& Yahr stage II or less and Mini-Mental State Examination (MMSE) score $\geq 24$ were used as inclusion criteria.

Results: PD patients were impaired relative to normal controls on the pro-saccades task. SRTs in the PD group (409.1 \pm 106 ms) were significantly longer than the HCs group (333.6 \pm 39.2 $\mathrm{ms})[\mathrm{t}(18.44)=-2.83, \mathrm{p}=0.011)]$. Percentage of anticipatory saccades were higher for PD (9.1) than for HCs (3.6) ( $\mathrm{U}=150.0$, $\mathrm{z}=-2.466, \mathrm{p}=0.014, \mathrm{r}=-0.36)$. Percentage of corrected response were higher for PD (3.6) than for HCs (1.8) ( $U=146.0, z=-$ 2.637, $\mathrm{p}=0.008, \mathrm{r}=-0.38$ ).

Conclusion: The specific deterioration of anticipatory and corrected saccade responses may reflect frontal-basal ganglia circuits that release the automatic saccade system from top-down inhibition in PD. Such detailed saccade assessments may be useful as a diagnostic preclinical screening tool for Parkinsonism.

Keywords: eye movements, Parkinson's disease, pro-saccade

\section{PP5-015}

Normative data from individuals aged $\mathbf{5 0}$ and over for visual event related P300 component

Yağmur Özbek $^{1}$, İlayda Kıyı ${ }^{1}$, Duygu Hünerli Gündüz, Deniz Yerlikaya $^{1}$, Ezgi Fide ${ }^{1}$, Görsev G. Yener ${ }^{2}$

'Department of Neurosciences, Institute of Health Sciences, Dokuz Eylül University, Izmir, Turkey; 'Izmir Biomedicine and Genome Center, Dokuz Eylül University Health Campus, Izmir, Turkey

Objective: The aim of this study was to provide P300 normative values elicited by visual oddball paradigm for a sample aged 50 and older.

Methods: One hundred and twenty-nine healthy volunteers were included. A classical visual oddball paradigm was used to elicit P300 component. In the paradigm, the white screen with the probability of $40 / 120$ and $40 \mathrm{~cd} / \mathrm{cm}^{2}$ luminance was applied as target stimuli. The white screen with the probability of $80 / 120$ and $10 \mathrm{~cd} / \mathrm{cm}^{2}$ luminance was used as standard stimuli. Independent Component Analysis (ICA) were performed in order to identify ocular artifacts, and the remaining artifacts were eliminated automatically. Mean P300 amplitudes for tar- get stimuli were automatically measured from $\mathrm{Fz}, \mathrm{Cz}, \mathrm{Pz}$ electrode locations in the $250-550 \mathrm{~ms}$ time window. The effects of age, education, and gender over P300 amplitudes were evaluated by multiple linear regression analysis.

Results: Multiple linear regression analysis indicated that the age is the only statistically significantly variable and its responsible for $4.4 \%, 5.8 \%$ and $5.3 \%$ of total variance in $\mathrm{Fz}, \mathrm{Cz}$ and $\mathrm{Pz}$ electrode locations, respectively. Age (3 Level: 50-59 years, 60-69 years, 70 years and over) was included in the model during ANOVA analysis. There was a main AGE effect on P300 amplitudes at $\mathrm{Fz}[\mathrm{F}(2.126)=3.651, \mathrm{p}=0.029], \mathrm{Cz}[\mathrm{F}(2.126)=$ $3.540, \mathrm{p}=0.032]$ ve $\mathrm{Pz}[\mathrm{F}(2.126)=3.301, \mathrm{p}=0.040]$. Further analyses have revealed that individuals between 60-69 years of age have higher P300 amplitudes than individuals aged 70 and over at all electrode locations

Conclusion: Normative data for visual P300 component in individuals aged 50 and over were presented for future use in studies. It is assumed that event-related P300 amplitude is an objective tool for in several clinical studies, especially in neurological and psychiatric investigations, as it is not affected by education, and gender.

Keywords: P300, normative data, EEG

\section{PP5-016}

Effects of open versus closed-skill exercise on cognitive functions and serum BDNF level

Evrim Gökçe ${ }^{1}$, Emel Güneş ${ }^{2}$, Fikret Ar1 ${ }^{3}$, Erhan Nalçacı ${ }^{2}$

'Physical Therapy and Rehabilitation Hospital, Ankara City Hospital, Ankara, Turkey; ${ }^{2}$ School of Medicine, Department of Physiology, Ankara University, Ankara, Turkey; ${ }^{3}$ Department of Electrical and Electronics Engineering, Faculty of Engineering, Ankara University, Ankara, Turkey

Objective: Exercise builds physical and functional changes in the brain. This status can affect cognitive skills and may be related to peripheral protein signals associated with cognition. The present study aims to examine the effects of open versus closed skill exercise on cognition and the brain-derived neurotrophic factor (BDNF).

Methods: Fencers (open-skill exercise) ( $\mathrm{n}=18)$, swimmers (closed-skill exercise) $(\mathrm{n}=18)$ and sedentary control group $(\mathrm{n}=18)$ were included in the study. 27 females and 27 males, right-handed participants are between the ages of 18-25. Visuospatial working memory was assessed with Corsi's Block Tapping Test and selective attention was assessed with Spatial Cueing Test. Serum BDNF basal level and its response to acute moderate aerobic exercise were analyzed. Statistical data were analyzed in SPSS v. 17 with ANOVA and Kruskal Wallis Test.

Results: Visuospatial working memory scores were significantly higher in the left hemispace for fencers compared to swimmers and sedentary $(\mathrm{p}=0.010, \mathrm{p}=0.000)$. Reaction times were significantly shorter in sports groups than sedentary controls on invalid trials in endogenous cueing test $(\mathrm{p}=0.000, \mathrm{p}=0.000)$. 
Reaction times were shorter for fencers compared to swimmers and sedentary controls on valid/invalid trials in exogenous cueing test $(\mathrm{p}=0.022, \mathrm{p}=0.003)$. BDNF basal serum level didn't show difference between the groups however, BDNF increased significantly in the sports groups compared to sedentary after acute exercise $(\mathrm{p}=0.001, \mathrm{p}=0.001)$. Women responded more to the exercise type in visuospatial working memory task and fencer women got higher scores.

Conclusion: Our findings demonstrated that open-skill exercise exhibited superior visuospatial working memory and selective attention especially for right hemisphere controlled areas than closed-skill exercise and sedentary. The results suggest that open-skill exercise including cognitively demanding activities may be preferred in planning a therapeutic approach and preventing neurodegeneration.

This research was supported by Ankara University Scientific Research Projects (18L0230004).

Keywords: BDNF, open-skill exercise, closed-skill exercise, working memory, attention

\section{PP5-017}

\section{Allogeneic platelet-rich plasma (PRP) preserves cognitive functions in aging brain}

\section{Mehtap Karagöz ${ }^{1}$, Enver Ahmet Demir ${ }^{2}$}

${ }^{1}$ Hatay Mustafa Kemal University, Faculty of Medicine, Hatay, Turkey; ${ }^{2}$ Department of Physiology, Faculty of Medicine, Hatay Mustafa Kemal University, Hatay, Turkey

Objective: Platelet-rich plasma (PRP) is blood product which is acquired by centrifugation of whole blood and incorporates higher concentration of platelets than the whole blood. PRP contains neurotrophic growth factors that promote cell regeneration. It's hypothesized in this study that PRP may be of benefit against the aging-related decline in cognitive activity which is characterized with the neural death, decrease in brain volume and loss of synaptic connections.

Methods: Forty-six elderly mice were used in the experiment. The cardiac blood was collected from thirty of those and high concentration platelet containing PRP was prepared. The remaining mice were divided to control $(n=8)$ and PRP $(n=8)$ groups. Allogeneic PRP was administered through the tail vein (i.v.) to the PRP group for 3 weeks. All subjects were undergone to behavioral and cognitive tests in the 4th week.Depending on whether the data are parametric or nonparametric, Student's t-test or Mann-Whitney U test was used for the comparison of the two groups. Statistical significance level (alpha) was set to $<0.05$.

Results: In the open field test, an increase in locomotor activity was observed in the PRP subjects $(\mathrm{p}=0.021)$. No significant difference was found between experimental groups in anxietylike behaviors in the open field and elevated plus maze tests ( $>0.05)$. The tail suspension test showed that there was no significance in depression-like behaviors among the subjects
( $>0.05$ ). The retention performance was found significantly better in the PRP group compared to the control group in the probe phase of the Morris water maze test $(\mathrm{p}=0.006)$.

Conclusion: The present study demonstrated that the intravenous administration of allogeneic PRP augments the locomotor activity in healthy aging mice. It's arousing that the PRP administration improves cognitive activity in the elderly mice. Also, it's noted that PRP does not result in anxiety- or depression-like behaviors.

Keywords: allogeneic, platelet-rich plasma, PRP, anxiety, depression, cognition

\section{PP5-018}

Multiple choice vs. open-ended questions: does it make a difference? An ERP study

Gülay Cedden ${ }^{1}$, Aykut Eken ${ }^{2}$, Giray Berberoğlu ${ }^{3}$

'Department of Applied Psychology, SRH University Heidelberg, Heidelberg, Germany; ${ }^{2}$ Pompeu Fabra University, Center for Brain and Cognition, Barcelona, Spain; ${ }^{3}$ Department of Psychological Guidance and Counseling, Faculty of Education, Başkent University, Ankara, Turkey

Objective: Assessment of cognitive skills in educational practice is a challenging problem. Even though the multiple-choice questions are extensively used in professional test programs, researchers criticise it because of its limitation to genuine learning and leading both teachers and students avoiding deep conceptual understanding of critical content. When the subject matter is considered two levels can generally be classified: explicitly and implicitly stated information. In this study, we tried to characterize the neural differences between solving questions which differ in question type (select/write) and level of cognitive processes (explicitly and implicitly stated information).

Methods: Event-Related Potentials (ERPs) from 19 electrodes were recorded while participants (N: 17, mean age: 21.1) were solving 48 questions. Stimulus material consisted of four question types: 12 select/explicit, 12 select/implicit, 12 write/explicit and 12 write/implicit (random order). The participants had to read a sentence after which a related question appeared. After having read the question the critical word select or write appeared on the screen for one second. ERPs were extracted and analysed at this period of initial response execution until they were asked to "Mark/Write the answer on the sheet".

Results: The mean amplitudes were compared using a two-way ANOVA with question type (select-write) $\times$ cognitive level (implicit/explicit) as independent factors. The write condition exhibited enhanced P300 amplitude at the parietal sensors (right- lateralized) compared to the select condition. When comparing the cognitive level a greater P300 was observed at the parietal channels for the implicit condition (left-lateralized). P300 amplitude is associated with attention, decisionmaking, context updating and level of cognitive resources. 
Conclusion: Our first results revealed that P300 might indicate that more attentional/cognitive resources are used when confronted with an implicit question that should be answered in written form. The correlation analyses between behavioural and ERP results are ongoing.

Keywords: neuroeducation, EEG, P300, implicit/explicit questions, multiple choice/open ended questions

\section{PP5-019}

An effect of adaptive memory on the stimulus differences in evaluating recall and familiarity

Gülsün Büşra Çiçek, Fatih Yurdalan, Oya Somer

Department of Psychology, Cyprus International University, Nicosia, Cyprus

Objective: Recollection involves two distinct processes, recalling and familiarity in dual-process theory. Even though survival processing enhances recognition, the effect of adaptive memory has not been found on familiarity in current researches. In this study, it was aimed to investigate the effect of the context and type of such stimulus on to what extent adaptive memory enhances the capacity of source memory for recalling and familiarity.

Methods: 45 healthy young adults participated in this study that was generated with a $2 \times 3$ mixed experimental design. Independent variables are face expressions (trustworthy, untrustworthy) and social attributions (collaborator, cheater, viewer), while dependent variables are reaction times and error rates. The paradigm was designed as three distinct parts. The survival scenario and instruction were given in the first part, In the second part, 6 names and social attributions belonging to faces were shown two times and then asked to be kept in mind for the recalling test. In the last part of the experiment, 3 tests consisting of recalling of names and social attributions along with familiarity test (if participants could not recall) were given.

Results: The responses given to the names belonging to trustworthy/collaborators were faster than others $(\mathrm{p}<.001)$. The response given to the social attributions belonging to trustworthy/collaborator and cheater were also found faster than viewer $(\mathrm{p}<.001)$. The accuracy rate $(0.57)$ for trustworthy/cheater faces in the social attribution recalling test was found the highest than others. The accuracy rate $(0.57)$ for the untrustworthy/cheater faces in the social attribution recalling test was also found the highest.

Conclusion: The results indicate that adaptive memory in recalling tests of social attributions has a stronger impact on cheaters than collaborators in both trustworthy and untrustworthy faces. Consequently, while participants gave the fastest reaction in trustworthy/cheater and collaborator, most recalled ones in social attributions were cheaters in both trustworthy and untrustworthy.

Keywords: adaptive memory, recall, familiarity, social attributions
PP5-020

Bilateral somatosensory stimuli elicit alpha activity while modifying long term memory performance

Gizem Göktepe, Tolga Esat Özkurt

Graduate School of Informatics, Health Informatics, Middle East Technical University, Ankara, Turkey

Objective: Bilateral somatosensory stimuli have been used to reveal episodic memories in a psychotherapy treatment method called eye-movement desensitization and reprocessing (EMDR). However, its effect on brain oscillations is not yet clear. In this study, we examined the effect of bilateral tactile stimuli on brain oscillations and long-term memory performance by designing novel old/new face recognition paradigm.

Methods: The experiment consists of two main parts: encoding and recognition sessions. In the encoding session, human face images comprising three ethnicities (Asian, Black, and White) were uniformly presented to the 22 healthy human subjects (mean age $=23.81 \pm 1.92$ ). In the recognition session, subjects were asked to recognize faces with somatosensory stimuli and without somatosensory stimulus in two separate sections. EEG recording was performed only in the recognition section using a 32-channel BrainAmp system with $1000 \mathrm{~Hz}$ sampling frequency. EEG raw data were bandpass filtered $(0.2-100 \mathrm{~Hz}$, 4th order Butterworth filter) and demeaned. Independent component analysis (fast ICA) was applied to identify and remove EOG artifacts. Time-frequency analysis was conducted using a Fourier basis with a fixed Hanning window length of $0.5 \mathrm{~s}$ (shifts of 50 $\mathrm{ms}$ ) in the frequency range $4-32 \mathrm{~Hz}$ with $2 \mathrm{~Hz}$ increments.

Results: Oscillatory analysis indicates that subjects who increased their memory performance with bilateral somatosensory stimuli have a significant alpha increase $(\mathrm{p}=0.007)$ in fronto-central channels prior to visual stimulus. Likewise, the left parieto-occipital channel has significantly greater alpha band activity $(\mathrm{p}=0.008)$ during the visual stimulus for recognition.

Conclusion: Our study shows that bilateral stimuli increase alpha activity in frontal channels for pre-stimulus and occipital channels for on-stimulus, which can be speculated to pave the way for eliciting long term memories. Preliminary findings suggest that bilateral somatosensory stimuli may change long term memory performance along with the modification of brain oscillatory activity.

Keywords: alpha band, long term memory, EEG, face recognition, neural oscillations

\section{PP5-021}

Neurophysiological investigation of dual N-back task with fNIRS and EEG hyperscanning

\section{Kerem Alp Usal, Murat Perit Çakır}

Department of Cognitive Science, Graduate School of Informatics, Middle East Technical University, Ankara, Turkey

Objective: It is common in everyday life that people need to perform an action together, which is called a joint action. Joint 
action requires extra effort compared to individual action, such as sharing mental representations, coordination, predicting partner's behavior, entrainment and perception-action matching, and these may affect cognitive workload. The cognitive mechanisms underlying joint action between individuals are still under research. Aim of this study is to shed light on neurophysological mechanisms of joint action.

Methods: Changes in neural activation during joint action was investigated with hyperscanning, using functional near infrared spectroscopy (fNIRS) and electroencephalography (EEG) as participants perform the same task individually and collaborate as a dyad. EOG and ECG data was also collected to investigate changes in blinking and heart rate. Interpersonal alignment of neurophysiological measures is also measured. The paradigm of this study was the N-back task with $0,1,2$ and 3 back levels.

Results: Sixty-two participants were tested in 31 same gender dyads. Statistical analyses were repeated-measures ANOVAs on n-back levels across task condition. Results showed that during the n-back task reaction times, error rates, heart rate and deoxyhemoglobin levels in PFC increased as n-back level increased whereas heart rate variability and oxyhemoglobin levels decreased, which is in line with the literature. Gender of the participants, which type of task they started with and whether the task was conducted by pressing the letter or numerical key did not have a significant effect on any of the measures.

Conclusion: The results regarding the effect of n-back levels are compatible with the literature. In the comparison of joint task to the individual task, higher deoxyhemoglobin levels were detected at right prefrontal cortex. This can be explained with exclusive task sharing, social facilitation or social loafing (Ringelmann effect). The analysis of EEG data is in progress and it will be followed with hyperscanning.

Keywords: Joint Action, Social Cognition, n-back task, functional near infrared spectroscopy

\section{PP5-023}

Is there an order effect on the mean P300 event related potential elicited by auditory and visual oddball paradigm?

İlayda Kıy1 ${ }^{1}$, İbrahim Öztura², Görsev Yener ${ }^{3}$

'Department of Neuroscience, Institute of Health Sciences, Dokuz Eylül University, Izmir, Turkey; 'Department of Neurology, Faculty of Medicine, Dokuz Eylül University, Izmir, Turkey; ${ }^{3}$ /zmir Biomedicine and Genome Center, Dokuz Eylül University Health Campus, , Izmir, Turkey

Objective: In this study, we examined the presence of order effect on the mean P300 event-related potential (ERP) elicited by auditory, and visual oddball paradigm in healthy individuals aged 50 and over.

Methods: There were demographically matched two groups consisting from 15 participants in each. The first group experienced the auditory classical oddball paradigm at first and then visual paradigm, whereas the second group paradigms did in the reverse order. The number of target/nontarget stimulus is
$40 / 80$ in both oddball paradigms. The auditory stimulus was 80 $\mathrm{dB}$; the tone was $1600 \mathrm{~Hz}$ for target stimuli and $1500 \mathrm{~Hz}$ for nontarget stimuli. In the visual oddball paradigm luminance of visual stimuli for target was $40 \mathrm{~cd} / \mathrm{cm}^{2}$, and for nontarget was $10 \mathrm{~cd} / \mathrm{cm} 2$. In both paradigms, all stimulus had a duration of $1000 \mathrm{~ms}$. The inter-stimulus interval varied between 3 and 7 seconds. In all EEG data, eye movements were corrected using Independent Component Analysis (ICA) method. EEG data were segmented as $1000 \mathrm{~ms}$ epochs that are $200 \mathrm{~ms}$ before and $800 \mathrm{~ms}$ after the target stimulus. Epochs containing muscle artifacts and other noise were automatically cleared and the rest were averaged for further analysis. The average P300 amplitude values (250-550ms) were measured automatically from the $\mathrm{Fz}, \mathrm{Cz}, \mathrm{Pz}$ electrode locations. Mixed ANOVA [Between Subject Factor: First Group, Second Group; Within Subject Factor: $\mathrm{Fz}, \mathrm{Cz}, \mathrm{Pz}]$ was used for statistical analysis which was performed by SPSS 22.0.

Results: There was no main group effect for auditory oddball paradigm $[\mathrm{F}(1.28)=0.312, \mathrm{p}=.58]$ and visual oddball paradigm [F $(1.28)=0.159, \mathrm{p}=.69]$.

Conclusion: We aimed to answer the methodological question that is there an order effect on mean P300 ERP elicited by auditory and visual oddball paradigm. We concluded there is no order effect for these paradigms.

Keywords: P300, event-related potential, oddball, auditory, visual

\section{PP5-024}

\section{Cognitive functions in stuttering}

Harun Ayas, Müge Müzeyyen Çiyiltepe, Ayşegül Yılmaz Department of Speech Language Therapy, Istinye University, Istanbul, Turkey

Objective: Stuttering is a speech fluency disorder that can be seen early or later in life by involuntary repetition and prolongation of sounds and syllables. Although there are various theories about its causes, recent studies are about the motor speech and cognitive outcomes of stuttering. In this study, the relationship between the severity of stuttering and the secondary behaviors that can be seen in stuttering as well as the accompanying phonological disorders with executive functions are emphasized.

Methods: The participants included 10 children who stutter (CWS) (6 males; age in months: 48-82; $\mathrm{M=64}$ ) and 11 children who do not stutter (CWNS) (6 males; age in months: 48-90; $M=66$ ) who spoke Turkish as their native language. The two groups were matched in age, phonological disorders (subtests of Turkish Articulation and Phonology Test) and nonverbal intelligence (indicated by Raven's Colored Progressive Matrices (RCPM)). Stuttering severity was estimated by calculating the percentage of syllables stuttered (\%SS) across the speech sample.

Results: According to the preliminary findings of the study, \%SS of the CWS was $10.1 \%$, and secondary behaviors were 
also accompanied (40\%). Average RCPM test results of CWS were 14.3 (min: 7-max: 21), and CWNS were 16,1 (min: 9, max: 27). The rate of phonological disorders in the participants was 76\% (CWS:70\%; CWNS:82\%).

Conclusion: In our study, in which preliminary findings were shared, it was found out that by measuring non-verbal skills such as problem solving and abstract reasoning (part of executive functions), individuals with stuttering may have secondary behaviors in addition to phonological components of their fluency, and phonological disorders can be observed independently of stuttering. It was concluded that the number of participants should be increased in future studies in order discuss statistical significance of the cognitive functions of both groups. Keywords: fluency disorders, cognitive functions, stuttering

\section{PP5-026}

\section{Developing an affective computational model for} RehabRoby: robot-assisted rehabilitation system

Elif Gümüsşı̈̈ ${ }^{1}$ Itır Kaşıkçı ${ }^{2}$ Yusuf Can Semerci ${ }^{3}$, Duygun Erol Barkana

'Department of Electrical and Electronics Engineering, Yeditepe University, Istanbul, Turkey; '2Department of Psychology, Istanbul Commerce University, Istanbul, Turkey; ${ }^{3}$ Department of Computer Engineering, Yeditepe University, Istanbul, Turkey

Objective: The goal of the project is to enable Rehabroby robot, which was developed to assist the rehabilitation of physical therapy patients, to recognize emotions of patients and to organize treatment process in accordance with patient's mood. To achieve this goal, we aimed to recognize emotion by deep learning from EEG signal.

Methods: 15 participants (10 female), aging between 20-23 were shown 72 positive, negative and neutral pictures $(24 \times 3)$ from International Affective Picture System (IAPS). Each picture was presented for 6 seconds. Brain electrical activity was recorded from 21 data channels using SmartBCI wireless EEG headset with a sampling rate of $250 \mathrm{~Hz}$. The artifact-free data was epoched to 1 -second slices. The slices then converted into image data by mapping the electrodes onto a matrix representing the location of the channels. The total energy of gamma, beta, and alpha bands obtained by using the 6th level wavelet decomposition were used as the RGB dimensions.

Results: Emotion classification was performed using classical Convolutional Neural Networks (CNNs) with 3 convolutional and three max-pooling layers which reduces dimensions of the data through abstraction. The number of filters used in each layer was 32 for all of the three layers. User-independent tranings were held by excluding one participant in each training and then giving that participant's data for test. The best classification accuracy values obtained are as follows: positive vs negative $56.28 \%$, positive vs neutral $53.02 \%$, negative vs neutral $51.52 \%$.

Conclusion: The emotion recognition results we obtained using IAPS stimulus set, from EEG signal, using CNN algo- rithm were quite lower than expected. That can be due to: Lilmited data, the stationary nature of the stimulus, the chaotic nature of the signal or converting the data into an image or using $\mathrm{CNN}$ algorithm not being ideal for this aim. Further studies are required.

Supported by TÜBA-Outstanding Young Scientist Awards (TÜBA-GEBIP).

Keywords: emotion recognition, EEG, deep learning, affective computing, robot-assisted rehabilitation systems

\section{PP5-027}

The effect of congruent and incongruent emotional expressions on emotion suppression task

Ayşe Nur Badakul, Dilara Steenken, Merve Nur Çevik, Yüksel Çiflik, Evrim Gülbetekin

Department of Psychology, Faculty of Medicine, Akdeniz University, Antalya, Turkey

Objective: In this study, we aimed to adapt the Stroop Test format to six universal emotional expressions by adding congruent or incongruent lexical emotional expressions on emotional faces. The participants were asked to mimic the facial expressions on the presented stimuli. The congruent (e.g. anger expression-"anger") and incongruent (happy expression"sadness") forms were created by combining emotional face expressions and written emotional expressions.

Methods: Stimuli were consisted of 6 emotional facial expressions and neutral facial expressions. A total of 49 stimuli with 7 emotional expressions of 3 male 4 female models were used. Twenty-two participants were involved. They completed two tasks: In the first task, they suppressed the facial expression on the photograph and express the emotion described by the word. In the second task they suppressed the word and expressed the emotion presented by the face. When the participants felt that they expressed the emotion they pressed the space key. We measured muscle activity of Corrugator Supercilii by using EMG and reaction time was recorded.

Results: The mean of reaction time for congruent and incongruent stimuli was found significantly different for the second task $[\mathrm{t}(21)=-2.80, \mathrm{p}=.011]$. When facial muscle activity was compared for congruent and incongruent stimuli, we observed that the EMG response for incongruent stimuli was lower than the congruent stimuli $(\mathrm{F}=7.97, \mathrm{p}=.01)$. Also a significant difference was found for fear expression when we compared EMG activity between two tasks $[\mathrm{t}(21)=2.44, \mathrm{p}=.02]$. The participants' EMG responses was higher when the expression of fear was presented lexically than it was presented facially.

Conclusion: Overall, the data indicated that incongruent stimulus presentation might have made the task more difficult and that may have caused a decrease in the muscle activity. Since fear is a vital emotion, the participants could have expressed fear by suppressing the other emotional expressions.

Keywords: emotional stroop, facial expressions, stroop test, emotion suppression 


\section{PP5-028}

Face recognition and metacognition: feeling of knowing judgments in response to familiar faces

Özlem Merve Cansever ${ }^{1}$, Metehan Irak ${ }^{2}$

'Department of Physiology, School of Medicine, Istanbul Aydın University, Istanbul, Turkey; ' Department of Cognitive Neuropsychology, Bahceşehir University, Istanbul, Turkey

Objective: In everyday life, we frequently experience the feeling which is "I know you but I do not remember your name". It is experienced especially when we encounter someone familiar, but we fail to remember the name of that particular person (Yarmey, 1973). In such situations, by monitoring our memory we make feeling of knowing judgments (FOK) about how likely we will remember the name of that person in future (Hart, 1965). We aimed to investigate the role of holistic face processing on face-recognition and FOK judgments.

Methods: Participants' $(n=60)$ face-naming and face-recognition performances as well as their FOK ratings were collected task under half-face (upper-half-face) and whole-face conditions during a semantic FOK which was developed by using the Psychopy Software.

Results: When subjects saw the whole-faces, they could significantly retrieve more identity-related information (e.g. occupation) compare to the half-face condition, $\mathrm{F}(1.28)=8.95, \mathrm{p}<.05$, $\eta 2=.24$. Participants who saw whole-faces was significantly more successful during face-naming than half-face group, $\mathrm{F}(1.28)=3.52, \mathrm{p}<.05, \eta 2=.11$. Retrieval of partial-information significantly improved the face-recognition performances when subjects saw whole-faces than half-faces, $F(1.28)=4.15$, $\mathrm{p}<.05, \eta 2=.13$. Half-face group significantly made lower FOK decisions for the faces that they could not retrieve any partial information, $\mathrm{F}(1.28)=5.56, \mathrm{p}<.05, \eta 2=.17$.

Conclusion: While basing FOK judgments, accessing identityspecific information (e.g. occupation) had effects on determining FOK decisions. Retrieving identity-specific information facilitated face-naming and face-recognition performances of participants. However, such effect was observed only under whole-face condition which in turn, supported holistic approach of face processing.

Keywords: face recognition, feeling of knowing judgments, metacognition

\section{PP5-029}

Short- and long-term forms of sensory plasticity probed with a motion adaptation paradigm

Sibel Akyüz $^{1}$, Andrea Pavan ${ }^{2}$, Utku Kaya ${ }^{3}$, Hulusi Kafalıgönül ${ }^{1}$

'Bilkent University, Interdisciplinary Neuroscience Program, Ayşe Sabuncu Brain Research Center \& National Magnetic Resonance Center, Ankara; ${ }^{2}$ Department of Psychology, University of Bologna, Bologna, Italy; ${ }^{3}$ Department of Anesthesiology, University of Michigan, Ann Arbor, MI, USA

Objective: Perception is shaped by both immediate pattern of sensory inputs and previous experience with the external envi- ronment. Visual adaptation, a temporary change in perception following exposure to a stimulus, has been widely employed to understand how previous sensory experience on different timescales shapes perception. Visual adaptation is considered a form of short-term neural plasticity. However, the neural mechanisms underlying these changes are still debated. In the present study, we aimed at discovering the spatiotemporal dynamics, neural substrates, and functional role of short-term sensory plasticity in the human visual system using a rapid motion adaptation paradigm combined with EEG. Specifically, we attempted at discovering how motion adaption-induced short-term sensory plasticity is reflected at the neural level and parallel with perceptual performance.

Methods: We adapted participants to directional drifting gratings for either short $(640 \mathrm{~ms})$ or long $(6.4 \mathrm{~s})$ durations and used a counter-phase flickering (i.e., directionally ambiguous) grating as a test pattern.

Results: Both adapting durations led to significant motion aftereffects, with the test pattern being perceived to drift in the opposite direction to that of the adaptation. However, the long adaptation produced stronger aftereffects than the short adaptation condition. In line with the behavioral results, short and long adaptation durations resulted in distinct aftereffects on the evoked activity within 64-112 ms time range over occipital and parieto-occipital sites (cluster based permutation tests, $\mathrm{p}=0.003$ ). More importantly, the aftereffects induced by the long adaptation condition were stronger than those induced by the short adaptation.

Conclusion: Taken together, these findings provide important electrophysiological evidence that motion aftereffects reflect changes in cortical areas mediating low- and mid- level visual motion processing. They also suggest that adaptation is an active process that involves neural mechanisms operating at different time scales.

Keywords: short-term adaptation, long-term adaptation, motion aftereffect, EEG

\section{PP5-031}

Developing an affective computational model for roborehab: assistant audiology rehabilitation robot

Elif Gümüşlü ${ }^{1}$, Bülent Koray Öz ${ }^{2}$, Talha Çögen ${ }^{3}$, Nuruefşan Üzmez ${ }^{4}$ Itır Kașıkçı ${ }^{4}$, Selma Yılar ${ }^{3}$, Hatice Köse ${ }^{5}$, Duygun Erol Barkana

'Department of Electrical and Electronics Engineering, Yeditepe University, Istanbul, Turkey; 'Department of Game and Interaction Technologies, Istanbul Technical University, Istanbul, Turkey; ${ }^{3}$ Department of Ear, Nose and Throat Diseases, Istanbul UniversityCerrahpaşa, Istanbul, Turkey; ${ }^{4}$ Department of Psychology, Istanbul Commerce University, Istanbul, Turkey; ${ }^{5}$ Department of Al and Data Engineering, Istanbul Technical University, Istanbul, Turkey

Objective: The goal of the project is to enable the Pepper robot, developed to assist the audiology tests of hearingimpaired children, to recognize children's emotions and to reg- 
ulate the behavior and feedback process in accordance with the child's mood. In order to achieve this goal, in this study, it is aimed to recognize emotion from physiological signals with machine learning.

Methods: While 9 children (6 male) ages between 4-6 were shown positive and negative emotional videos and a neutral one as control, skin conductance (SC), blood volume pulse (BVP) and temperature (TEMP) were obtained using E4 Wristband. For machine learning, the average, variance and mean of the first derivative of all three signals were determined as features. For additional features, heart rate (HR), heart rate variability (HRV) were calculated from the BVP signal, and the HRV spectrum was divided into three ranges: very low (VLF) $(0-0.05 \mathrm{~Hz})$, low $(\mathrm{LF})(0.05-0.15 \mathrm{~Hz})$ and high frequency $(\mathrm{HF})$ $(0.15-0.4 \mathrm{~Hz})$. For feature preparation; metric extraction, classification and clustering preprocessing steps were applied. Machine learning algorithms have been implemented using the Weka program. While processing physiological data, the attempt was to find a system where maximum success is achieved by selecting the calculated features, algorithm and classification type in different combinations.

Results: The best results (around 80\%) were obtained with KStar and Random forest algorithms using LF, HF, LF/HF, average BVP, variance of BVP and first order derivative of BVP for neutral-negative, and with SVM algorithm using LF, $\mathrm{HF}$ and LF/HF metrics for neutral-non neutral classifications. High results were not obtained from SC or TEMP.

Conclusion: Among BVP, SC and TEMP physiological data, BVP provides the best features for emotion recognition from physiological signals. Different algorithms for different classifications (neutral-negative or neutral-non neutral) provide maximum success $(80 \%)$.

This project was supported by TUBITAK (Project no: 118E214).

Keywords: emotion recognition, physiological signals, machine learning, affective computing, robot-assisted rehabilitation systems

\section{PP5-033}

Spatial attention modulates surround suppression

${\text { Merve Kınıklığ }{ }^{1}}^{1}$, Hüseyin Boyacı ${ }^{2}$

'Interdisciplinary Neuroscience Graduate Program, Bilkent University, Ankara, Turkey; '2Department of Psychology, Bilkent University, Ankara, Turkey

Objective: The normalization model of attention predicts that spatial attention affects surround suppression as the narrower top-down gain increases the effect of excitatory drive and causes weaker suppression (Reynolds and Heeger, 2009). Macaque studies confirmed that directing attention to the center results in weaker suppression, whereas directing attention more spatially broadly increases suppression (Reynolds and Chelazzi, 2004). We investigated the effect of attention on surround sup- pression by using a motion direction discrimination task with two different conditions: narrow attention (NA) and wide attention (WA).

Methods: We foveally presented drifting sinusoidal gratings with a fixed size (diameter: $1.5^{\circ}$ ). The grating was presented alone in NA condition or surrounded by either a $2.5^{\circ}$ or $9.2^{\circ}$ thick annulus in both conditions. The area between $\left(1.5^{\circ}\right.$ to $2.8^{\circ}$ ) remained unstimulated. Center and surround gratings either moved in the same direction or different direction. In NA condition, participants were asked to attend the center and report its motion direction. In addition to that question, in WA condition, we also asked participants whether the center and surround moved in the same direction to encourage them to widen their attention field. In each trial, the duration of the presentation was adjusted based on the responses of the six observers following an adaptive procedure.

Results: When center and surround moved in the same direction, we observed the expected effect of surround suppression; thresholds increased as the stimulus size increased $(\mathrm{p}<.001)$. Importantly, surround suppression was significantly stronger in the WA condition vs. NAs regardless of the size and contrast ( $\mathrm{p}<.001)$. Thus, as the window of spatial attention expanded, duration thresholds increased dramatically.

Conclusion: In line with animal studies, we found that increasing the spatial extent of the attention evokes stronger suppression. These results suggest that neural suppression in the visual cortex may also be stronger when attention is wider.

Keywords: center-surround interaction, spatial attention, surround suppression

\section{PP5-034}

The effect of Xbox 360 Kinect games on cognitive skills of multiple sclerosis patients

Nilay Özen, Selen Gür Özmen

Department of Neuroscience, Institute of Health Sciences, Bahçeşehir University, Istanbul, Turkey

Objective: In this study the effect of Xbox360 Kinect, which is a virtual reality application, was examined on Multiple Sclerosis (MS) patients to assess their cognitive skills.

Methods: Patients diagnosed with Relapsing Remitting and Primer Progressive MS were included in the study. Patients were selected from a private clinic according to inclusion criteria. Before the intervention neuropsychological tests (NPT) of the patients were done by following tests; Line Orientation Test, Öktem Verbal Memory Test, Digit Span Test, Verbal Fluency Test, Stroop Test, Trial Making Test, PASAT, Beck Depression Inventory, Beck Anxiety Inventory. Results were recorded for comparison after intervention. Patients played previously determined Xbox360 Kinect games for 5 weeks, 2 days a week, 1 hour each. At the end of 5 weeks, patients were subjected to NPT again and the results of pre and post-intervention were compared. 
Results: According to preliminary results, an improvement in the information processing speed, attention, long-short term memory, working memory, motor speed, visual scanning speed, cognitive flexibility, response inhibition, mental recall and mood values were observed. However, there was no improvement in the verbal and visual attention, concentration, visuospatial perception as expected.

Conclusion: Our research, which is one of the few studies that exemplify the use of video games for neuro-rehabilitation purposes other than entertainment, has brought positive results. It is an easy and enjoyable application method that people can easily use in their home without going to a center. Thus, it provides an advantage in terms of accessibility and applicability. Rehabilitation with virtual reality games is expected to be applied to other neurological diseases in the future.

Keywords: Xbox, multiple sclerosis, cognition

\section{PP5-035}

The effects of nine different types of ASMR sounds on recognition

Gizem Tanseli Kaspar

Department of Cognitive Sciences, Yeditepe University, Istanbul, Turkey

Objective: Autonomous Sensory Meridian Response is an activation through the sounds of swallowing, drying hair, breathing, or natural and mechanical sounds such as raining or clicking on a keyboard. ASMR sound stimuli could be in auditory or visual format. This study aims to analyze the possible effect of the ASMR sounds on recognition items as opposed to playing no sound under identical conditions.

Methods: In order to see the effect of ASMR sounds on memory, we had 30 participants take part in a recognition task under no sound condition, and ASMR sound condition in which the participants were exposed to three subsets of ASMR sounds (human, mechanic and nature sounds). We conducted our experiment in VR Lab at Yeditepe University. Participants were exposed to ASMR sounds condition and no sound condition while they were on a memory task.

Results: Data sheets were examined in SPSS with One-way ANOVA. There was a significant effect between no sound and ASMR sounds on recognition, $\mathrm{F}(1.58)=25.40$, $\mathrm{p}=.00$ The participants performed better in the recognition task when they were not exposed to any ASMR sounds. Also, there was a significant difference between ASMR sounds in 3 different conditions (human sounds, mechanical sounds and nature sounds), $\mathrm{F}(3.587)=11.97, \mathrm{p}=.00$. The participants showed poor memory performance when they were exposed to human ASMR sounds than mechanic and nature ASMR sounds.

Conclusion: The findings of the study revealed a significant difference between the performance of the participants under ASMR sound condition and no sound condition. The ASMR sounds did not enhance the participants' memory performanc- es also when we compared the ASMR sound conditions in between showed the same results. Further study can be conducted on which neural mechanisms activates or suppresses by ASMR sounds, which increases or decreases the performance in the memory test.

Keywords: Autonomous Sensory Meridian Response (ASMR), tingling, recognition, sensory processing, memory

\section{PP5-037}

Effect of music performance on memory and language

Sayeste Cağ

Department of Physiology, Faculty of Medicine, Ankara University, Ankara, Turkey

Objective: Constant music performance leads to activity in several brain areas and causes structural and functional differences. The hypothesis of cross-modal transfer of plasticity suggests that these differences may change cognitive functions. Based on this hypothesis, effect of music performance on memory and language was investigated by using neuropsychiatric tests which were not used for this literature.

Methods: 30 musicians and 30 control subjects were selected regarding their answers in Musical Past Questionnaire, Hand Preference Questionnaire and General Participant Questionnaire. Amateur and professional musicians who play a musical instrument actively for at least 5 years were admitted. For both groups, only right hand users were included. Ages apart from 18-40, psychiatric and neurological disorders and related medication use, not being in the follicular phase in menstruation cycle for female participants, were in the exclusion criteria. Cognitive assessment of working memory, visuospatial memory, executive functions and language were done by using Corsi Block Tapping Test (CBTT), Mental Rotation Test (MRT), Trail Making Test (TMT) and Verbal Fluency Test (VFT).

Results: There was no significant differences between groups regarding age, gender, hand preference and education $(\mathrm{p}>0.05)$. Statistical analysis of data showed that there were no significant difference between groups regarding CBTT, MRT and TRT, hence working memory or visuospatial memory $(p>0.05)$. However, in VFT, control participants significantly made more mistakes than the musicians $(\mathrm{p}<0.05)$.

Conclusion: Results showing that there are no significant difference between groups regarding the CBTT, MRT and TRT, contradicts with the literature. This was based on the tests used which were thought to be not distinctive enough and the difference between groups would be significant with modification to these tests. For VFT, higher error scores in control group shows that executive function and inhibition is affected by music performance. This is a new finding for VFT and is consistent with literature.

Keywords: cognitive, language, memory, music, neuropsychiatric test 


\section{PP5-040 \\ Evaluation of the relationship between metacognitive functions and mathematical reasoning skills of high school students diagnosed with attention deficit and hyperactivity disorder with behavioral and neurocognitive methods \\ Ecem Okurgan \\ Department of Psychology, Istanbul Gelişim University, Istanbul, Turkey}

Objective: This study was carried out to evaluate the relationship between metacognitive functions and mathematical reasoning skills of high school students diagnosed with ADHD, behavioral and neurocognitive methods, and to learn about the relationship between metacognitive functions and mathematical reasoning skills.

Methods: Personal information and Sociodemographic information form, Mathematical Reasoning Skill Determination Scale and Metacognition Scale were distributed to 110 high school students aged 14-17 years with ADHD. Cross-sectional descriptive research model was used and the collected data was transferred to the SPSS program program and interpreted with statistical analysis.

Results: According to the result of anova test conducted to determine whether there is a positive low level relationship between metacognition level and mathematical reasoning skill level according to the result of pearson correlation analysis ( $\mathrm{p}=0.019 ; \mathrm{r}=0.220)$.; It was found to have a statistically significant difference $(\mathrm{F}=13.801, \mathrm{p}=0.000, \mathrm{p}<0.01)$. It was determined that the mathematical reasoning skill levels of those whose age was 14 years old $(=64.27)$ were higher than those who were 16 years old $(=90.42)$ and 17 years old $(=90.00)$. Scheffe test was used for which groups the difference originated. When the reliability coefficients of metacognition levels consisting of 28 items were examined, it was determined that the reliability level was high (Cronbach's Alpha $=0.893>0.70)$. When the reliability coefficients of the level of mathematical reasoning skills consisting of 35 items were examined, it was determined that the level of reliability was high (Cronbach's Alpha= 0.744>0.70).

Conclusion: The results show that there is a positive relationship between metacognition level and mathematical reasoning skill level, which means that when there is an increase in students' metacognition level, there will be an increase in mathematics reasoning skill levels.

Keywords: ADHD, metacognition, mathematical reasoning

\section{PP5-042}

The effect of vision on top-down modulation of hand blink reflex

Fatma Zehra Çalıkuşu, Ayşegül Gündüz, Meral Erdemir Kızıltan Department of Neurology, Cerrahpaşa Medical Faculty, Istanbul UniversityCerrahpaşa,, Istanbul, Turkey

Objective: The magnitude of hand blink reflex (HBR) increases when a threatening stimulus is positioned in the peripersonal space (PPS) compared to the stimulus in the extrapersonal space (EPS). We hypothesized that the reflex increase in PPS may change depending on whether the stimulus is seen or not. Here, we aimed to investigate the alterations in HBR response to understand the effects of vision on cortical modulation of HBR.

Methods: The HBR was recorded from 11 healthy volunteers while the hand was far away from the face (EPS), close to face with eyes open (PPS-eyes open), and close to face with eyes closed (PPS-eyes closed). Changes in the response magnitudes were compared between the three conditions.

Results: HBR was obtained in all subjects. As expected, there was an increase in the reflex magnitude in the PPS-eyes open condition relative to EPS. In the PPS-eyes closed condition, the duration and latency was shorter and area-under-the-curve was significantly smaller compared to the PPS-eyes open condition.

Conclusion: The enhancement of HBR in PPS is attributed to the tonic top-down modulation. Our study provides evidence for the special sensory modulation of the PPS effect on HBR and may suggest cortical modulation of top-down modulation of brainstem neural circuits.

Keywords: hand-Blink reflex, peripersonal space, vision

\section{PP5-045}

Effects of visuo-tactile stimulation on face perception

Evrim Gülbetekin $^{1}$, Seda Bayraktar ${ }^{1}$, Deniz Kantar ${ }^{2}$, Enes Altun ${ }^{1}$, Nurullah $\mathrm{Er}^{1}$, Arda Fidanc1 ${ }^{1}$

'Department of Psychology, Akdeniz University, Antalya, Turkey; 2Department of Biophysics, Akdeniz University, Antalya, Turkey

Objective: The aim of the presented study is to find out if tactile stimulation on one's own face has any effect on face processing by using event related potentials (P300).

Methods: Ten undergraduate students participated the study. Fourty facial stimuli (20 female, 20 male) were presented in the aqusition stage. The participants observed the faces in the first session of the study, while in the second session a robotic finger was touched on their left cheeck for 3s. The order of the sessions was counterbalanced between subjects. After the acquisition phase, the subjects were tested in a face memory task. During the acquisition and test phases, brain signals were recorded via 64channel EEG system. RT and accuracy were recorded.

Results: ERPs were averaged $200 \mathrm{~ms}$ before (-200ms) and $1000 \mathrm{~ms}$ after stimulus onset. The amplitude was measured at occipital, parietal occipito-parietal, temporal and frontal electrode sites (O1, O2, P3, P4, P7, P8, PZ, PO3, PO4, PO7, PO8, F1, F2, F3, F4, F5, F6, F7, F8, T7, T8, TP7, TP8, TP9, TP10). $\mathrm{P} 300$ (250-500ms) responses at these electrode sites were examined while the subjects were observing faces. Mean amplitudes of electrode regions were analyzed by conducting repeated measures ANOVA. The results indicated that there was a significant difference among electrode sites during observation of faces $\mathrm{p}<.05$. The amplitudes of P300 in parietal, occipital and PO sites were higher than that of amplitudes in the other sites. On the 
other hand, significant effects of electrode site $\mathrm{p}<.05$, hemisphere $\mathrm{p}<.05$. and interaction of site and hemisphere $\mathrm{p}<.05$ were found on P300 responses during tactile stimulation. The amplitudes in frontal and temporal sites were significantly higher than that of amplitudes in the other sites $\mathrm{p}<.05$. The accuracy between two conditions was also different $\mathrm{p}<.05$.

Conclusion: Overall, the findings have shown that being touched on one's self-face had a significant effect on processing of other faces.

Keywords: face perception, tactile stimulation, face memory, ERP

\section{PP5-046}

Effect of adipokinetic hormone on learning and memory in scopolamine induced Alzheimer model in mice

İbrahim Uygun, Oğuz Mutlu, Faruk Erden

Department of Pharmacology, Kocaeli University, Kocaeli, Turkey

Objective: Neurosecretuary cells of corpus cardiacum of insects synthesis adipokinetic hormone (AKH). Our previous studies showed that chronic administration of $\mathrm{AKH}$ to rats exerted memory improvement in experimental schizophrenia and depression models. This study investigated effects of AKH on learning-memory in scopolamine induced Alzheimer model.

Methods: Morris water maze (MWM), passive avoidance (PA) and modified elevated plus maze (mEPM) tests used for evaluation of cognitive functions. 120 male balb-c mice were used. Groups were control, Ani-AKH (1 and $2 \mathrm{mg} / \mathrm{kg})$, Lia-AKH (1 and $2 \mathrm{mg} / \mathrm{kg}$ ), Pht-HrTH (1 and $2 \mathrm{mg} / \mathrm{kg}$ ), Scopolamine (1 $\mathrm{mg} / \mathrm{kg}$ ), Scopolamine+Ani-AKH (1 and $2 \mathrm{mg} / \mathrm{kg}$ ), Scopolamine+ Lia-AKH (1 and $2 \mathrm{mg} / \mathrm{kg}$ ), Scopolamine+Pht-HrTH (1 and 2 $\mathrm{mg} / \mathrm{kg}$ ). In MWM test hormones administered 6 days to evaluate effects on learning-memory; in PA test hormones administered before second trial to evaluate effects on memory and in $\mathrm{mEPM}$ test hormones administered after first trial to evaluate effects on consolidation.

Results: In MWM test at the end of 5th day, Ani-AKH $2 \mathrm{mg} / \mathrm{kg}$ and Pht-HrTH $2 \mathrm{mg} / \mathrm{kg}$ decreased escape latency increasement in scopolamine group $(\mathrm{p}<0.05)$. In MWM test, in probe trial, Ani-AKH ( 1 and $2 \mathrm{mg} / \mathrm{kg}$ ) increased time spent in escape platform's quadrant compared to control $(\mathrm{p}<0.05)$; and reversed effect of scopolamine $(\mathrm{p}<0.05 ; \mathrm{p}<0.001$; respectively). In probe trial Lia-AKH and Pht-HrTh didn't alter time spent in escape platform's quadrant compared to control while they reversed effect of scopolamine $(\mathrm{p}<0.01)$. In PA test, Ani-AKH ( 1 and 2 $\mathrm{mg} / \mathrm{kg})$ reversed effect of scopolamine $(\mathrm{p}<0.05)$ while in $\mathrm{mEPM}$ test, Lia-AKH $2 \mathrm{mg} / \mathrm{kg}$ reversed effect of scopolamine $(\mathrm{p}<0.01)$.

Conclusion: Our results showed that effects of hormones were test dependent. In MWM test, Ani-AKH exerted positive effects on normal memory; Ani-AKH and Pht-HrTH showed improvement in disturbed learning while all hormones improved disturbed memory. In PA test, Ani-AKH improved disturbed memory while in $\mathrm{mEPM}$ test Lia-AKH improved disturbed consolidation.

Keywords: adipokinetic hormone, memory, mice, scopolamine

\section{PP5-047}

The spatial spread of the tilt aftereffect

Büșra Tuğçe Gürbüz ${ }^{1}$, Hüseyin Boyacı ${ }^{2}$

'Department of Psychology, Bilkent University, Ankara, Turkey; ${ }^{2}$ National Magnetic Resonance Research Center (UMRAM), Bilkent University, Ankara, Turkey

Objective: The tilt aftereffect (TAE) is observed when prolonged exposure to a tilted contour causes subsequently presented straight contour to be perceived as tilted to the opposite direction. Although extensive research has been done on TAE, how it spreads across the visual field has not been systematically studied before. Thus, this study aims to fill this gap.

Methods: Here, we measured the magnitude of TAE at 15 peripheral locations. During the experiment, participants fixated a central fixation mark. In an experimental trial, 5000 milliseconds adaptation to a Gabor patch tilted clockwise by 15 degrees followed by a 200 milliseconds blank screen and 100 milliseconds presentation of the test grating. The adapter was presented always at the same location in an experimental block, in either visual hemifield, at an eccentricity of $10.5^{\circ}$ along the horizontal meridian. Test gratings were presented in 15 different locations in the same hemifield, including the adapter location. Left and right hemifield tests were counterbalanced across participants. Participants indicated the direction of the tilt of the test grating by pressing the right or left arrow button on a keyboard. Control conditions were identical to experimental conditions except there was no adaptation. Subjective verticality of the test grating was determined by using an adaptive procedure and fitting logistic regression function to the data. TAE magnitude was calculated by subtracting the control conditions' thresholds from experimental conditions' thresholds.

Results: We found a significant spread of TAE across the whole visual hemifield $(\mathrm{N}=19, \mathrm{p}<0.05)$ except one location (down meridian, $2.5^{\circ}$ eccentricity) $(\mathrm{p}=0.472)$, showing that TAE is not limited to adapted location in visual hemifield.

Conclusion: These results can provide critical novel information about the functional organization of visual cortex which can be explained further by modeling. Moreover, the spatial extent of other aftereffects that share similar neuronal mechanisms can be tested for future directions.

Keywords: tilt aftereffect, spatial spread, orientation adaptation

\section{PP5-048}

An anatomical review: the afferent and efferent pathways of the nucleus accumbens

Hilal Akdemir Aktaș, Zehra Çelik, Ceren Günenç Beşer

Department of Anatomy, Faculty of Medicine, Hacettepe University, Ankara, Turkey

Objective: The nucleus accumbens is an important component of the ventral striatum. It is a round and flattened struc- 
ture, which is located anterior to the posterior border of the anterior commissure and lies paralel to the midline. It has a crucial role in the cognitive processing of motivation, reward, addiction, aversion, slow-wave sleep and reinforcement learning. The aim of this study was to revisit the afferent and efferent pathways of the nucleus accumbens.

Methods: A comprehensive literature review was performed using Pubmed and Google Scholar websites.

Results: Neurons in the nucleus accumbens receive input from the ventral tegmental area (via the mesolimbic dopaminergic pathway), the amygdaloid complex (primarily via the ventral amygdalofugal pathway), from the hippocampal formation (through the precommissural fornix), and from cells of the bed nucleus of the stria terminalis. Additionally, fibers from the prefrontal cortex and prelimbic cortex project to the nucleus accumbens. Efferent projections of the nucleus accumbens include fibers to the hypothalamus, the ventral tegmental area, the substantia nigra, amygdala, the subpallidal region and the globus pallidus. The afferent and efferent pathways of the nucleus accumbens has been implicated in numerous neurological and psychiatric disorders, including depression, obsessivecompulsive disorder, bipolar disorder, drug addiction, Parkinson, Alzheimer and Huntington diseases.

Conclusion: The nucleus accumbens is a complex and fascinating structure that has great influence over a variety of human behaviors. Knowing afferent and efferent pathways of the nucleus accumbens will guide to the physicians for diagnosis and treatment of the neurological and psychiatric disorders.

Keywords: nucleus accumbens, afferent pathway, efferent pathway, ventral tegmental area, amygdaloid complex

\section{PP6}

Sleep, Autonomic and Neuroendocrine Systems

\section{PP6-001}

Literature review of physiopathological changes seen in intracranial structures in insomnia

Nurullah Yücel $^{1}$, Betül Cetintulum Huyut ${ }^{2}$

'Department of Anatomy, Hamidiye Medicine Faculty, University of Health Sciences, Istanbul, Turkey; ${ }^{2}$ stanbul Arel University, Institute of Social Sciences, Clinical Psychology Doctorate Program, Istanbul, Turkey

Objective: Insomnia; is defined as difficulty in falling asleep or maintaining sleep, which causes impairment in mental or physical functionality in daily life. Prevalence rate of insomnia is $10-15 \%$. The aim of this study is to determine which anatomical structures are in the foreground in insomnia patients.

Methods: The structures of the nervous system which were affected by chronic insomnia, has been investigated by conducting a literature review. In the recent studies, the anatomical regions that are emphasized in common in the data obtained by MRI were identified and summarized.
Results: While the functional connection in the left and right middle-frontal-orbital, left-opercular inferor-frontal gyrus and left-angular regions are decreased in patients with chronic insomnia, also the functional connection in the right middlecingulate-gyrus, right-hippocampus, right-cuneus, right-paracentral lobe and right-inferior temporal-gyrus are increased. Gender-differences are observed in the cerebellum, limbiclobe, left-premotor cortex and left-dorsolateral prefrontal cortex of insomnia. It is thought, varying sudden activities in important functional networks such as the insular-cortex and the left-thalamus area of the hyperarousal network are associated with insomnia. Decreased gray matter concentrations in the dorsolateral-prefrontal and pericentral-cortex, superiortemporal gyrus, and cerebellum, and also decreased gray matter volume in the medial-frontal and temporal regions are observed in insomnia. It is thought that the gray matter deterioration in the pericentral and lateral temporal regions of insomnia may be related to the difficulties in the initiation and continuation of sleep.

Conclusion: As a result of the literature review of recent years on insomnia; It has been determined that there are differences according to gender in the central nervous system. The most affected anatomical formations in insomnia are; Inferiorfrontal gyrus, middle-temporal gyrus, superior-temporal gyrus, inferior-temporal gyrus, cingulate gyrus, hippocampus, cerebellum and thalamus. Detailed investigation of these anatomical regions in patients with a pre-diagnosis of insomnia may guide clinicians.

Keywords: insomnia, neuroanatomy, nervous system structures.

\section{PP6-002}

\section{Effects of $2600 \mathrm{MHz}$ radio-frequency radiation on cerebellum in rats with and without melatonin supplementation}

Sinem Oruç $^{1}$, Kevser Delen ${ }^{1}$, Dilek Kuzay ${ }^{2}$, Bahriye Sırav ${ }^{1}$

'Department of Biophysics, Faculty of Medicine, Gazi University, Ankara, Turkey; 'Department of Physiology, Faculty of Medicine, Kırşehir Ahi Evran University, Kırşehir, Turkey

Objective: After the increased risk in glioma cases in epidemiological results of INTERPHONE Project from International Agency for Research on Cancer, Radio-frequency Radiation (RFR) put in the class2B which means the agent is possible carcinogen. The usage of mobile phones is rapidly increasing in our daily lives and cell phones can be classified as a source of RFR. In present study, it was aimed to investigate the effect of $2600 \mathrm{MHz}$ RFR exposure on MDA (malondialdehyde), GSH (glutathione) and NO (nitricoxide) levels and the protective role of melatonin in cerebellum tissues of male rats.

Methods: 36 male Wistar albino rats were randomly divided into 6 groups;Control, Sham, RFR, Control+Melatonin, Sham+Melatonin, RFR+Melatonin. Control animals were kept 
in their usual cages during whole experiment. In Sham group, the exposure system was kept off and similar procedures were applied with the RFR group. RFR group;2600MHz RFR exposure was applied with $21.74 \mathrm{~V} / \mathrm{m}$ for 30 minutes/day,30days. Melatonin injections were performed as $10 \mathrm{mg} / \mathrm{kg}$, sc for 30 days in melatonin groups. At the end of 30 days, MDA, GSH, NO levels were measured in the cerebellum tissues of rats by spectrophotometer.

Results: No difference was observed between the control and sham groups $(\mathrm{p}>0.05)$. It was observed that NO and MDA levels were significantly higher in the RFR group with respect to (w.r.t.) control and sham groups, and GSH levels were significantly lower $(\mathrm{p}<0.05)$. NO and MDA levels were significantly lower in RFR+Melatonin group w.r.t. RFR group and GSH levels were significantly higher $(\mathrm{p}<0.05)$. NO and MDA levels were significantly higher in RFR+Melatonin group w.r.t. Control+Melatonin and Sham-Melatonin groups and GSH levels were significantly lower $(\mathrm{p}>0.05)$. No significant difference was observed between Control+Melatonin and ShamMelatonin groups ( $>00.05)$.

Conclusion: Short term RFR exposure leads to an increase in oxidative stress and lipid peroxidation in the rat cerebellum, possible preventive measures should be taken by further investigation.

The study was supported by Gazi University BAP Unit (01/2018-05).

Keywords: radiofrequency radiation, melatonin, cerebellum, oxidative stress, rat

\section{PP7}

Neural Networks and Computational Neuroscience

\section{PP7-002}

\section{ETS transcription factor family in Drosophila neurogenesis}

$\underline{\text { Pinar Mutlu }}^{1}$, Ekin Sönmez ${ }^{2}$ Yiğit Koray Babal ${ }^{2}$, Işıl Kurnaz ${ }^{1,2}$ 'Department of Molecular Biology and Genetics, Gebze Technical University, Kocaeli, Turkey; ${ }^{2}$ Institute of Biotechnology, Gebze Technical University, Kocaeli, Turkey

Objective: ETS transcription factor family has an important role in regulation of some cellular processes such as proliferation, differentiation, apoptosis and cell migration. This transcription factor family is quite large and members are grouped according to their similarities in their ETS domain which is about 85 amino acids as PEA3, ETS, ERG etc. Although the activation of target genes by ETS proteins occurs through specific protein-protein interactions, the mechanism of ETS transcription factors in target gene selectivity cannot be fully determined. In this study, we aim to dissect Drosophila ETS transcription factor and their target genes in neurogenesis by combining bioinformatics tools.
Methods: In this study, ETS related RNAseq datasets and neurogenesis related transcriptomic datasets were obtained from GEO database. Differentially expressed genes (DEGs) were detected in ETS datasets and downstream analysis such as Gene Ontology (GO) analysis was performed. After the GO analysis, nervous system related genes were determined. Expression levels of these selected DEGs were examined by neurogenesis dataset. The promoter sequences of target genes were detected by using EPD database and ETS binding motif determined by using JASPAR database.

Results: Drosophila ETS transcription factors regulate the genes in neurogenesis. According to promoter analysis, a gene regulatory network was established between ETS genes and their neurogenesis related target genes.

Conclusion: Our study showed that, ETS transcription factors, specially Pnt has role in Drosophila development. In light of our findings, our regulatory network should be compared with mammalian datasets then a therapeutic approach for neurodegenerative disease may developed by merging different model systems.

Keywords: drosophila, ETS transcription factors, neurogenesis, Pnt

\section{PP7-003}

Drug agents of "4B05" protein and "Curcuma longa" plant actives associated with Alzheimer's disease

Ayşenur Öztürk ${ }^{1}$, Kubilay Göçücü ${ }^{2}$, Didem Dost ${ }^{3}$

${ }^{\prime}$ Department of Computational Science and Engineering, Institute of Informatics, Istanbul Technical University, Istanbul, Turkey; ${ }^{2}$ Department of Medical Biology and Genetics, Institute of Health Sciences, Marmara University, Istanbul, Turkey; ${ }^{3}$ Department of Molecular Biology and Genetics, Faculty of Arts and Sciences, Acibadem Mehmet Ali Aydınlar University, Istanbul, Turkey

Objective: Alzheimer's disease $(\mathrm{AD})$ is a neurodegenerative disease that usually affects the elderly. Beta-secretase 1 (BACE1), which cleaves-site amyloid precursor protein, is an enzyme involved in the formation of amyloid $\beta$ peptide $(A \beta)$ species. Since the cerebral accumulation of $A \beta$ species may be a critical factor in the pathogenesis of Alzheimer's disease, BACE1 has become a target for the treatment of this disease.In the study of Fredrik Jeppsson et al., a coupling study with AZD3839 (PDB ID: 4B05) protein, a potential inhibitor associated with BACE1, was performed. This study is a reference that the relevant enzyme (BACE1) is effective in the pathogenesis of the disease. It is aimed to compare the binding energies of the candidate plant with the drug molecules used for AD. A calculation study has been carried out with the forms of Curcuma longa plant.

Methods: Information on the actives and drug molecules of Curcuma longa was obtained from PubChem. The best conformers were obtained in Spartan's 14V1.1.4 (Wavefunction Inc, 2006) program. Later, the $\mathrm{x}, \mathrm{y}, \mathrm{z}$ coordinates of the selected protein, 4B05, were determined in the Biovia Discovery 
Studio Visualizer (Dassault Systèmes BIOVIA, 2017) and its ligands were deleted.Using Autodock Vina(v1.1.2) program, docking study of drug molecules and plant actives to $4 \mathrm{~B} 05$ protein was performed.

Results: The binding energy of 1-HYDROXY 1.7-BIS (4HYDROXY 3-METHOXYPHENYL) and 2-HYDROXY METHYL ANTHRAQUINONE was determined as -8.0 $\mathrm{kcal} / \mathrm{mol}$.The binding energy of 1,7-BIS (4-HYDROXY PHENYL) HEPTA 1,4,6-TRIENE 3-ONE was calculated as $-8.3 \mathrm{kcal} / \mathrm{mol}$ whereas donepezil, memantine, rivastigmine, which are the drug molecules used in $\mathrm{AH}$, were found to be $8.3,-5.9,-6.3 \mathrm{kcal} / \mathrm{mol}$, respectively.

Conclusion: The binding energies of the plant actives and donepezil,memantine,rivastigmine which are currently in use for AH were compared.It has been argued that herbal actives could potentially be medicinal. When the calculation results are analyzed 1.7-BIS-(4-HYDROXY PHENYL) HEPTA 1, 4, 6 TRIENE 3-ONE, 1-HYDROXY 1.7-BIS (4-HYDROXY 3 METHOXYPHENYL) 6-HEPTENE 3.5 DIONE and 2HYDROXY METHYL ANTHRAQUINONE plant actives were observed to exhibit a binding energy equal to donepezil and better than other pharmaceutical actives.

Keywords: Alzheimer's disease, computational neurochemistry, curcuma longa, docking

\section{PP7-004}

Feature regularization in voxelwise modeling for naturalistic fMRI experiments

Özgür Yilmaz ${ }^{1}$, Emin Çelik², Tolga Çukur ${ }^{3}$

'National Magnetic Resonance Research Center, Bilkent University, Ankara, Turkey; ${ }^{2}$ Neuroscience Program, Bilkent University, Ankara, Turkey; ${ }^{3}$ Department of Electrical and Electronics Engineering, Bilkent University, Ankara, Turkey

Objective: Voxelwise modeling (VM) is a powerful framework to predict single-voxel functional selectivity for the stimulus features that exist in complex natural stimuli. Yet, because VM disregards potential correlations across stimulus features or neighboring voxels, it may yield suboptimal sensitivity in measuring functional selectivity in the presence of high levels of measurement noise.

Methods: We introduce a novel voxelwise modeling approach that simultaneously utilizes stimulus correlations in model features and response correlations among voxel neighborhoods. The proposed method performs feature and spatial regularization while still generating single-voxel response predictions. We demonstrated the performance of our approach on an fMRI dataset from a natural vision experiment implemented on five healthy male subjects.

Results: Compared to classical voxelwise modeling, the proposed method yields clear improvements in prediction performance, together with increased feature coherence and spatial coherence of voxelwise models. We find that the proposed method significantly improves prediction scores compared to classical method in most of the examined regions-of-interest ( $<<0.05$, Bootstrap test). Compared to existing methods, the proposed approach better captures feature and spatial coherence in cortical representations, while improving prediction accuracy.

Conclusion: Our results indicate clear improvements in prediction performance, spatial coherence and feature coherence of category models in a natural vision experiment, specifically across visual category areas and attention-control areas. This work is supported by National Eye Institute Grant (grant number EY019684), by a Marie Curie Actions Career Integration Grant (grant number PCIG13-GA-2013-618101), by a European Molecular Biology Organization Installation Grant (grant number IG 3028), by a TUBA GEBIP 2015 fellowship, by a BAGEP 2017 fellowship, by an ASELSAN PhD scholarship, by a TUBITAK-BIDEB 2211 scholarship, and by an NVIDIA GPU Grant.

Keywords: computational neuroscience, feature regularization, modeling, stimulus correlation

\section{PP7-005}

Dynamic time warping (DTW) as a feature in classification of motor task fNIRS data

Ekin Can Erkus, Vilda Purutçuoğlu

Middle East Technical University, Ankara, Turkey

Objective: The applicability of dynamic time warping (DTW) as a feature is mainly aimed to be investigated in a pairwise classification study of fNIRS data with three motor tasks: Finger tapping of left (LHFT) and right hand (RHFT) and foot tapping (FT). For the applicability of DTW algorithm as a feature, it is expected to increase in the accuracy of labelling the tasks by involving DTW as a feature.

Methods: DTW is tested as a feature which represents the non-linear warped similarity value between the samples and mean baseline for classification of fNIRS data in this study. The data are acquired from a publicly available web repository, which are collected from 30 subjects by 20 channels during 3 different motor tasks in an experimental paradigm. Data mean, variance, skewness, kurtosis and range values are used as the main features with and without DTW values to classify the grand averages of the channels corresponding to the LHFT, RHFT and FT. K-NN classifier with Manhattan distance and $\mathrm{k}=2$ is used as the classifier and leave-one-out method is used as the validation method.

Results: The classification accuracy of the oxygenated hemoglobin (oxy-HB) levels between RHFT and LHFT increases from $68.3 \%$ to $73.3 \%$; RHFT and FT increases from $73.3 \%$ to $81.7 \%$; LHFT and FT increases from $71.7 \%$ to $81.7 \%$ by involving DTW as a feature. On the other hand, the classification accuracies of the deoxygenated hemoglobin (deoxy-HB) levels have no significant change by involving DTW as a feature. 
Conclusion: The usage of DTW is found to cause a general increase in the classification accuracies of fNIRS data between the grand averages corresponding to RHFT, LHFT and FT motor tasks for oxy-HB measures. The reason might be the distinctive non-linear data behaviour of the oxy-HB levels for the corresponding motor tasks.

Keywords: feature extraction, dynamic time warping, classification, fNIRS, motor task

\section{PP7-006}

Detection of myocardial ischaemia by using sympathetic nerve activity, artificial neural network and Gaussian mixture model

Merve Begüm Terzi, Orhan Arıkan

Department of Electrical and Electronics Engineering, Bilkent University, Ankara, Turkey

Objective: A new technique which detects anomalies in skin sympathetic nerve activity (SKNA) and ECG by using state-ofthe-art signal processing and machine learning methods is developed to perform robust detection of myocardial ischemia (MI).

Methods: Proposed technique is developed by using STAFF III database composed of raw recordings acquired before and during coronary balloon angioplasty performed in 104 coronary artery disease patients by using data acquisition equipment with wide frequency bandwidth and high sampling rate. Database is constructed as part of clinical research study conducted by Charleston Medical Center to investigate induced ECG anomalies that occur during MI. A signal processing technique which simultaneously obtains SKNA and ECG from wideband recordings on STAFF III database is developed. A novel feature extraction technique which obtains ECG features that are most discriminative for reliable detection of $\mathrm{MI}$ is developed. A novel supervised learning technique based on artificial neural network (ANN) which performs robust detection of MI is developed. An unsupervised learning technique based on Gaussian mixture model (GMM) and Neyman Pearson approach which performs robust detection of MI using only basal ECG data is developed.

Results: Findings of study demonstrated that there is increase in amplitude of SKNA during MI which is accompanied by simultaneous repolarization anomalies in ECG. It is shown that anomalies which occur in SKNA during MI are associated with ST/T anomalies in ECG. Performance results of proposed ANN-based supervised learning technique over STAFF III database showed that technique provides highly reliable detection of MI with $89.7 \%$ accuracy and $85.2 \%$ specificity by performing robust detection of ECG anomalies.

Conclusion: In cases where diagnostic information of ECG is not sufficient for reliable diagnosis of $\mathrm{MI}$, proposed technique can provide early and accurate diagnosis of MI, which can lead to a significant reduction in mortality of ischemic heart diseases.

Keywords: artificial intelligence, artificial neural network, feature extraction, gaussian mixture model, neurology, signal processing

\section{PP7-007}

The effect of prior knowledge on visual processing of agents: time-resolved representational similarity analysis on EEG data

Hüseyin Orkun Elmas ${ }^{1}$ Sena Er ${ }^{1}$, Ayşe Pınar Saygın², Burcu Ayşen Ürgen ${ }^{1}$

'Graduate School of Science and Engineering, Department of Neuroscience, Bilkent University, Ankara, Turkey; ${ }^{2}$ Department of Cognitive Science, UC San Diego, La Jolla, CA, USA

Objective: Visual processing of actions involves identification of the agent who performs the action and

the type of action. In the presented study, we investigated how prior knowledge affects temporal characteristics of neural processing during perception of videos and still images that include various agents and actions.

Methods: We conducted two EEG experiments with human participants. In both experiments, participants were presented videos and images of 3 agents performing 8 actions while EEG was recorded. In the first experiment (Prior), participants $(n=19)$ were familiarized with the stimuli and informed about the identity of the agents before the experiment. In the second experiment (Naive), participants $(n=16)$ were naive to the identity of the agents. EEG data was preprocessed with standard procedures including filtering, referencing, and artifact rejection. To investigate the time course of agent and action processing, we linked the EEG data in a time-resolved manner to two categorical models, Agent and Action models, using representational similarity analysis. We then performed multiple regression analysis by including Agent and Action models as well as two low-level stimulus feature models as predictors to reveal the unique contribution of Agent and Action models to EEG data.

Results: Our regression analysis with RSA shows that when subjects had no prior knowledge about agents, agent information was available for a longer duration during processing of images (66-406ms) than videos $(92-128 \mathrm{~ms}) \quad(\mathrm{p}<0.01$, Bonferroni corrected for all time points). However, when subjects had prior knowledge about the agents, the difference between the still images and videos was diminished. We did not find any time interval in EEG data when Action information was available ( $\mathrm{p}<0.01$, Bonferroni corrected for all time points).

Conclusion: In conclusion, prior information affects temporal characteristics of visual processing of agent perception by modulating the onset and duration of when agent information is available.

Keywords: EEG, representational similarity analysis, agent perception, action perception, prior knowledge 
PP8

Neuroimaging and Neuromicroscopy

\section{PP8-001}

Neural activation for object-based selection persists despite random changes in object features

Esra Nur Catak ${ }^{1}$, Hulusi Kafalıgönül ${ }^{2}$, Mert Özkan ${ }^{3}$, Gene Stoner $^{4}$

'Interdisciplinary Neuroscience Program, Bilkent University, Ankara, Turkey; ${ }^{2}$ National Magnetic Resonance Research Center (UMRAM), Bilkent University, Ankara, Turkey; ${ }^{3}$ Department of Psychological and Brain Sciences, Dartmouth College, Hanover, NH, USA; ${ }^{4}$ Salk Institute for Biological Studies, La Jolla, CA, USA

Objective: Mounting evidence indicates that selective attention spreads within objects, enhancing the processing of taskirrelevant features. Various aspects of this object-based attention were studied extensively using the transparent motion design (Valdes-Sose et al., 2000) and its variants (Reynolds et al., 2003). In this paradigm, when subjects are asked to judge brief translations of one of two superimposed dot fields, they were able to judge motion direction of the cued dot field more accurately.

Methods: Stoner and Blanc (2010) later discovered that the cueing advantage was specific to the individual cued dots and not due to neuronal adaptation and competition by introducing color and motion direction swaps of the dots at the onset of the translation. We investigated the neural basis of these behavioral findings with an EEG paradigm based on the previous design with delayed-onset cueing and with motion and/or color swaps.

Results: Our behavioral results $(n=15)$ confirmed that even though it was reduced by motion swaps $(\mathrm{p}=0.004)$, the cueing effect was still significantly specific to the cued dots $(\mathrm{p}<0.001)$. A cluster-based permutation test on the ERPs revealed a spatiotemporal cluster around $200-320 \mathrm{~ms}$ time range $(\mathrm{p}=0.003)$ which was centered over occipital and parieto-occipital scalp sites. To determine whether the components identified by this analysis were regulated by cueing and/or swapping, we performed a two-way repeated-measures ANOVA with cueing (cued vs. uncued) and swapping (no-swap, motion, color, motion, and color) as factors on the averaged ERP amplitudes. We found a strong cueing effect $(\mathrm{p}<0.001)$ and swapping had no overall significant effect.

Conclusion: Our findings revealed that the cueing effect, even in the presence of motion and color swaps, is specific to the individual dots and associated with the modulations of neural visual processes.

Support: This research was supported by the Scientific and Technological Research Council of Turkey (BIDEB 2221 and 2211-E Programs).

Keywords: electroencephalography (EEG), object-based attention, visual processes

\section{PP8-002}

Spatial synchronization patterns within intrinsic connectivity networks in resting-state fMRI

Hüden Neșe ${ }^{1}$, Ali Bayram², Tamer Demiralp², Ahmet Ademoğlu

'Department of Biomedical Engineering, Boğaziçi University, Istanbul, Turkey; ${ }^{2}$ Department of Neuroscience, Aziz Sancar Institute of Experimental Medicine, Istanbul University, Istanbul, Turkey

Objective: The intrinsic connectivity networks (ICNs) of the brain are described by the temporal correlations observed in the low-frequency $(0.01-0.1 \mathrm{~Hz})$ fluctuations of the blood oxygenlevel dependent (BOLD) signal among brain regions, which reveals distinct spatial maps that putatively correspond to specific sensory, motor or cognitive brain networks. We investigated the spectral characteristics of the major ICNs by taking into consideration the spatial synchronization pattern within each network.

Methods: We used the minimally preprocessed MSMAll ICAFIX cleaned data of 96 participants (53 female) from the Human Connectome Project (Glasser et al., 2013). The cortical data were parcellated according to Scheafer's atlas (400 parcels, 7 networks) (Scheafer et al., 2018). For each network, two spectral estimates were computed by Welch method applied on BOLD time series. The first one was obtained by averaging the power spectral densities estimated for each parcel of a network, corresponding to spectral distribution of the total power in the network, and the second by estimating power spectral density of the averaged time series of all parcels corresponding to the synchronization within the network. We defined the ratio of the synchronized by total power as the Spatial Synchronization Index (SSI) and compared it among 7 networks by cluster mass permutation and ANOVA tests.

Results: The ANOVA on whole frequency range showed significant within-network synchronization differences among 3 main groups of networks $[\mathrm{F}(6.665)=164.3, \mathrm{p}<.01]$ : sensory (visual, somatomotor), attention (dorsal attention, salience ventral attention) and higher order cognitive (default mode, control and limbic). Higher order cognitive networks had significantly lower SSI values compared with sensory and attention networks. Cluster mass permutation tests showed that the two attention networks differed from each other specifically in the $0.055-0.01 \mathrm{~Hz}$ range.

Conclusion: Distinct spectral synchronization patterns among ICNs may provide new insights about their internal mechanisms and inter-network relationships.

Keywords: fMRI, Resting state Networks, Synchronization patterns

\section{PP8-003}

The association between neural correlates of time perception and reward anticipation in patients with major depressive disorder

Simge Altınok ${ }^{1}$, Nihal Apaydın², Sertaç Üstün ${ }^{3}$, Emre Hikmet Kale ${ }^{4}$, İpek Çelikağ ${ }^{4}$, Halise Devrimci Özgüven ${ }^{5}$, Bora Baskak ${ }^{5}$, Metehan Çiçek 
'Department of Interdisciplinary Neuroscience, Ankara University, Ankara, Turkey; ${ }^{2}$ Department of Anatomy, School of Medicine, Ankara University, Ankara, Turkey; ${ }^{3}$ Department of Physiology, School of Medicine, Ankara University, Ankara, Turkey; ${ }^{2}$ Brain Research Center, Ankara University, Ankara, Turkey; ${ }^{5}$ Department of Psychiatry, School of Medicine, Ankara University, Ankara, Turkey

Objective: Patients with major depressive disorder (MDD) indicated distortions in time perception. Timing is affected by the presence of reward and changes in reward magnitudes. In MDD, there are dysfunctions in brain structures involved in the reward system which may alter the perceived time through overlapping neural mechanisms. This study aimed to investigate the neural correlates between the brain structures in which time perception and reward anticipation are represented in MDD patients.

Methods: We used a different method of analysis to examine the data of another study that had previously been conducted. Participants comprised 17 healthy adults and 7 MDD patients. The use of drugs affecting dopaminergic and noradrenergic systems and comorbid conditions were involved in the exclusion criteria for patients. Participants performed a time perception task in which they had to estimate whether the moving object reappeared earlier or later after the obstruction according to its anticipated speed in "reward" and "no-reward" sessions. Subjects attended to the color tone change of object in the control condition. In reward sessions, participants received a monetary reward per correctly done trials in order to evaluate brain activity during rewarding. The fMRI data were acquired with 3T scanner and analyzed using SPM12. The study had approval from AUIRB.

Results: Time perception showed an activation difference in superior temporal gyrus between healthy adults and MDD patients $(\mathrm{F}=13.15 ; \mathrm{p}<0.005)$. Furthermore, reward anticipation led to activation difference in inferior parietal lobule between groups $(\mathrm{F}=12.06 ; \mathrm{p}<0.005)$. Time perception and reward anticipation interacted in bilateral SMA $(\mathrm{F}=17.58 ; \mathrm{p}<0.005 ; \mathrm{F}=15.06 ; \mathrm{p}<0.005)$; however the integration of time with reward has been differentiated with activations in SMA ( $\mathrm{F}=20.38$; $\mathrm{p}<0.005)$, IPS $(\mathrm{F}=18.13$; $\mathrm{p}<0.005)$ and thalamus $(\mathrm{F}=15.39 ; \mathrm{p}<0.005)$ between groups.

Conclusion: These results suggest that functional differences related to the interaction of timing with reward in MDD may occur through cortico-striatal and frontoparietal networks.

Keywords: time perception, reward anticipation, major depressive disorder, fMRI.

\section{PP8-004}

Longitudinal analysis of brain networks by magnetic resonance imaging (MRI) in a murine model of neuropathic pain-induced depression

Meltem Karataş ${ }^{1}$, Vincent Noblet ${ }^{1}$, Laetitia Degiorgis ${ }^{1}$, Muris Humo ${ }^{2}$, Marion Sourty ${ }^{1}$, Julien Lamy ${ }^{1}$, Thomas Bienert ${ }^{3}$, Jean Paul Armspach ${ }^{1}$, Dominik von Elverfeldt ${ }^{3}$, Ípek Yalçın², Laura Adela Harsan'

'Strasbourg University, ICube Laboratories, Strasbourg, France; ${ }^{2}$ Strasbourg University, INCl, Strasbourg, France; ${ }^{3}$ Department of Medical Physics, Freiburg University, Freiburg, Germany
Objective: Chronic pain conditions frequently lead to anxiety and depressive disorders. Despite considerable clinical research, the mechanisms underlying this comorbidity remain elusive. We conducted a non-invasive in vivo brain imaging study to investigate changes in structural and functional connectivity in a mouse model of neuropathic pain-induced depression.

Methods: We employed two methods of magnetic resonance imaging (MRI) to investigate functional communication pathways (using resting state functional MRI- rs-fMRI) as well as their microstructural substrates (diffusion MRI) in a longitudinal manner in the cuff model. We scanned the same cohort of neuropathic and control mice at baseline, 2 weeks after the nerve injury and at 8 weeks where we observe anxio-depressive behaviours. Seed-based functional connectivity (FC) analysis and the analysis of diffusion tensor metrics were performed in relevant datasets. Differences between the groups and between the timepoints were shown using a GLM framework, at significance cutoff $\mathrm{p}<0.05$ (FWER correction applied at cluster level).

Results: Structural imaging showed transient changes in painprocessing regions at 2 weeks and more stable modifications in ACA and RSP at both timepoints, with lower FA values in these regions for neuropathic group. rs-fMRI revealed considerable changes in the networks encompassing the reward circuit (higher FC between VTA, LHb and ACB) and DMN (between ACA and RSP) in neuropathic mice. These brain areas are known to be involved in both chronic pain pathologies and major depression.

Conclusion: Brain networks demonstrate remarkable structural and functional modifications following the induction of neuropathic pain and the emergence of depressive phenotype. The long-term perspective of this project is to investigate the causal relationship between pain and depression, reaching a mechanistic explanation for the comorbidity.

Keywords: brain connectivity, depression, mouse model, MRI, neuropathic pain

\section{PP8-007}

Morphometric comparison of temporal bone structures in patients with unilateral tinnitus

Kübra Peker ${ }^{1}$, Ahmet Kağan Karabulut ${ }^{1}$, Zeliha Fazlıŏulları ${ }^{1}$, Çağdaş Elsürer ${ }^{2}$, Mehmet Öztürk ${ }^{3}$, Nadire Ünver Doğan ${ }^{1}$

'Department of Anatomy, Faculty of Medicine, Selçuk University, Konya, Turkey; 'Department of Otorhinolaryngology, Faculty of Medicine, Selçuk University, Konya, Turkey; ${ }^{3}$ Department of Radiology, Faculty of Medicine, Selçuk University, Konya, Turkey

Objective: Tinnitus is a common neuro-otological disease for which exact information about its cause and treatment has not been reached yet. In this study, making morphometric measurements of os temporale in patients and healthy ears of patients with unilateral tinnitus; It was aimed to compare these measurements according to gender, side, patient-health status, and bulbus jugularis levels. 
Methods: In the study, 30 (18 females, 12 males) patients with unilateral subjective non-pulsatile tinnitus had meatus acusticus internus (MAI) diameter, length, n. cochlearis bone canal diameter (NCKC), bulbus jugularis (BJ) diameter and level, fenestra vestibuli niche height (FVNY) os temporale computed tomography (CT) images were measured. The data obtained were compared according to gender, side, health status and bulbus jugularis levels.

Results: In the results obtained, the diameter of MAI in the sick ears was found to be statistically significantly smaller on the right side from the left side. When the ears with left and right tinnitus were compared with same person's other ear, a statistically significant difference was observed. BJ diameter was significantly different only in healthy ears with hypotympanic and mesotympanic BJ ears. When bulbus jugularis levels were investigated, 43 hypotympanicand 17 mesotympanic BJs were seen, while no epitympanic located BJ was observed. FVNY was found to be significantly smaller in the left ears tinnitus than in the right ears tinnitus ears. When individuals with left tinnitus were compared with their healthy right ears, it was observed that the left side was significantly smaller than the right.

Conclusion: Significant differences between the right and left sides of the ears with tinnitus suggest that the right and left sides may affect tinnitus in different degrees. The results obtained from the study will help to interpret the CT images of patients with tinnitus, determine the etiology, and create a treatment plan.

Keywords: anatomy, CT, morphometry, Os temporale, tinnitus

\section{PP8-008}

Morphometric analysis of projection fibers in Alzheimer's patients by web-based MRICloude method

\section{Meryem Esma Düz ${ }^{1}$, Nurullah Yücel ${ }^{2}$, Muzaffer Şeker $^{1}$}

'Department of Anatomy, Faculty of Medicine, Necmettin Erbakan University, Konya, Turkey; ${ }^{2}$ Department of Anatomy, Health Sciences University, Istanbul, Turkey

Objective: In the literature, a high neuroinflammation has been observed in the posterior part of the capsula interna in $\mathrm{AD}$. As the severity of $\mathrm{AD}$ increased, a significant decrease was observed in the fornix fractional anisothropy value measured by Diffusion Tensor Imaging.We aimed to determine whether the projection fibers have undergone any change in their volumes due to the observation of changes in projection fiber structures in studies on Alzheimer's patients.

Methods: T1 3D MR images of a total of 21 people with 10 Alzheimer's disease and 11 control group (CG) were used. The MRICloud program provides 5 different tables about the results. We chose this because the table containing the data of our study is in the Type2 L5 statistics table.

Results: Capsula interna sinister/dexter, Corona radiata sinister and dexter, Fornix sinister/dexter, Capsula externa dexter/sinister volume measurements were found to be higher in CG data than in AD. However, no statistically significant dif- ference was found ( $\mathrm{p}>05)$. The volume measurements of capsula externa sinister were higher in the CG $(2957,454 \pm 295,31)$ than in the $\operatorname{AD}(2655,500 \pm 225,79)$ and this was found to be statistically significant $(\mathrm{p}<05)$.

Conclusion: The statistical difference in capsula externa sinister measurement results can be interpreted as the fact that capsula Interna contains both projection and association fibers. Our study has shown that Alzheimer's disease has no effect on projection fibers.Studies involving more patients are needed to clarify this result.

Keywords: Alzheimer's disease, MRICloude, projection fibers

\section{PP8-009}

\section{Classification of EEG data by graph signal processing}

Sevde Büșra Bayrak, Ahmet Ademoğlu

Department of Biomedical Engineering, Boğaziçi University, Istanbul, Turkey

Objective: Mechanism of the human brain is of primary importance to understand the basis of neurological and behavioral problems. Studying the brain electrical activity called the Electroencephalography (EEG) using graph signal processing techniques are becoming more widely used to understand the behavior of the brain networks. The goal of this study is to analyze the EEG signals obtained under two different emotional conditions as the 13 subjects experience a series of randomly mixed 280 pleasant and 280 unpleasant pictures selected from the IAPS dataset with the mean valence level differing (7.13/2.96), but the mean arousal level being equal for the two sessions (4.99/5.02)

Methods: EEG data is recorded from 30 channels according to the International 10-20 electrode placement system. Each electrode location is mapped onto a unit sphere as a graph node and the resulting graph is used to compute the adjacency matrix by identifying the nearest neighbors of each node. The graph Laplacian matrix is obtained by taking the difference between the degree and adjacency matrices upon which the graph spectral decomposition is performed. Data is projected to a subspace determined by graph eigenvalues and their graph eigenvectors of graph Laplacian so between the two conditions is achieved using a classification algorithm. The data is also projected to the temporal frequency subspaces determined by Fourier components defined by conventional EEG bands as delta, theta, alpha, and beta.

Results: It is observed that, without any graph spectral decomposition, both SVM and KNN algorithms do not achieve an accuracy higher than $60 \%$ across the frequency bands. On the other hand, the classification accuracy increases to around $90 \%$ when graph spectral decomposition is applied before classification.

Conclusion: Results indicate that when EEG spatio-temporal signal is analyzed, its spatial brain network features captured by graph spectral components yield important information on data classification.

Keywords: electroencephalography, support vector machines, K-nearest neighbor, graph signal processing 


\section{PP8-010}

The role of theta response in the process of distinguishing one's own body

Samet Çelik, Rümeysa Büşra Doğan, Cennet Sena Parlatan, Bahar Güntekin

Graduate School of Health Sciences, Program of Neuroscience, Istanbul Medipol University, Istanbul, Turkey

Objective: The body recognition process includes complex visual processing, the sensation, perception and distinction stages of the stimulus. This study examines the process by using time-frequency analysis of EEG signals and analyzed the data by using event-related oscillations method. Our aim is to examine the theta response during the process of healthy individuals' distinguishing their own bodies from other bodies.

Methods: Seventeen young, adult, with a mean age of $21.94 \pm 2.01$ years, within normal BMI, who had no known psychiatric or neurological diseases, were included. They were asked to distinguish their own bodies among a set of five stimuli. The EEGs were collected using 32-channel EEG caps. The band limits of the recording were between 0.1 and $250 \mathrm{~Hz}$. The sampling rate was $500 \mathrm{~Hz}$. The impedances were below 10 $\mathrm{k} \Omega$ and the earlobes were taken as reference electrodes. Eventrelated spectral perturbation (ERSP) method was used for EEG data analysis. The Repeated-Measures ANOVA analysis was the statistical method.

Results: The Repeated-Measures ANOVA analysis reported no significant difference in theta response between 0 and 400 $\mathrm{ms}(\mathrm{p}>0.05)$ but the main effect of the stimulus was found statistically significant in the prolonged theta response between 400-800 ms F(1.16)=7.991, p=0.012, 0.33. The post-hoc comparison reported a higher theta response in recognizing one's own body $(3.395 \pm 0.367)$ compared to recognizing the other bodies $(2.594 \pm 0.236)$. Location $\mathrm{x}$ hemisphere interactions were not statistically significant ( $\mathrm{p}>0.05)$.

Conclusion: A significant prolonged theta response was previously reported in the process of distinguishing one's own body from other bodies. The statistical non-significance of the interaction effects supports our hypothesis that this process does not occur in a single location. As far as is known, this is the first study that temporally demonstrates the distinction process of the stimulus (one's own body) which requires hierarchical processing.

Keywords: body recognition, EEG, event-related oscillations, theta

\section{PP8-011}

Relationship between neural networks related to time, space and number perception: a meta-analysis of neuroimaging studies

Burcu Sırmatel $^{1}$, Hazal Şimşek ${ }^{2}$, Beyza Doğanay Erdoğan ${ }^{3}$, Metehan Çiçek ${ }^{2}$

'Department of Interdisciplinary Neuroscience, Ankara University, Ankara, Turkey; ' Department of Physiology, Ankara University, Ankara, Turkey; ${ }^{3}$ Department of Biostatistics, Ankara University, Ankara, Turkey
Objective: Perceptions of time, space and number have been studied extensively, and there have been an increasing number of neuroimaging studies on these topics in recent years. 'A theory of magnitude' (ATOM) was created with the thoughts that there may be some commonalities in the neural basis of these three perceptions and to explain their representation in the brain. However, there are also opinions that these perceptions may be processed on completely separate bases. Although there are studies and metaanalyzes that evaluate two of these three perceptions together, there is no meta-analysis study that evaluates all three perceptions together. The aim of our study is to investigate whether these perceptions are represented by common neural networks.

Methods: The studies included in the meta-analysis were selected on the following criteria: articles published in a peerreviewed journal, containing only healthy participants, containing 10 or more participants, from 2000 to present and on functional resonance imaging (fMRI) studies. (However, prefinding from 2000 to 2010 are given in this report.) The articles were searched on PubMed. The data of the included studies were evaluated using the GingerALE program.

Results: Time perception activation likelihood estimation (ALE) analysis showed activation in the right inferior frontal gyrus (IFG), right intraparietal sulcus (IPS), bilateral insula, bilateral supplementary motor area (SMA), and left temporal gyrus. Right IFG, right IPS and bilateral peristriate cortex showed activation for spatial perception, while bilateral IFG, left dorsolateral prefrontal cortex (DLPC), bilateral IPS/superior parietal lobe (SPL) showed activation of bilateral insula for number perception. Analysis for three perceptions showed bilateral insular cortex, bilateral IFG, left DLPC, bilateral SMA, bilateral IPS/SPL, and bilateral peristriate cortex activation.

Conclusion: The preliminary findings of our study suggest that a neural network consisting of prefrontal-parietal and premotor-insular cortex carries out the magnitude processes necessary for space-time-number perception and supports the ATOM theory.

Keywords: fMRI, magnitude perception, number perception, space perception, time perception

\section{PP9}

Nervous System Diseases and Treatment Approaches

\section{PP9-002}

Alterations in motor functions of rats after systemic delivery of genes encoding native or mutant transactive response DNA-binding protein-43 (TDP-43)

Elif Polat Corumlu $^{1}$, İlkim Büyükgüdük ${ }^{1}$, Çınar Furkan İlhan ${ }^{1}$, Handan Açelya Kapkaç², Muhittin Arslanyolu², Emel Ulupinar ${ }^{3}$

'Department of Interdisciplinary Neuroscience, Health Sciences Institute, Eskişehir Osmangazi University, Eskişehir, Turkey; 'Department of Molecular Biology, Faculty of Sciences, Eskişehir Technical University, Eskişsehir, Turkey; ${ }^{3}$ Department of Anatomy, Faculty of Medicine, Eskişehir Osmangazi University, Eskişehir, Turkey 
Objective: Transactive response-DNA binding proteinopathy (TDP-43) has become a hallmark feature in motor neuron diseases. Not only overexpression, but also deletion of the nuclear localization signal (NLS) causes proteinopathic alterations. We employed viral vector mediated gene transfer method for delivery of genes encoding native or mutant TDP-43 to assess motor functions of young adult male and female Sprague-Dawley rats.

Methods: Motor neuron specific targeting of AAV9 vectors were achieved by using a cell type specific promoter (UCHL1). While control animals received SF injection $(n=10)$, experimental groups were injected by either native or NLS-deleted viruses $(1.77 \times 1012 \mathrm{vg} / \mathrm{kg}, \mathrm{n}=7 /$ each group), on postnatal day 30. Following systemic injection of viruses, motor coordination of animals was evaluated by Rota-rod and modified grip tests for 4 weeks. Then, spatial learning and memory abilities of animals were measured by Morris Water Maze (MWM) test.

Results: The number of falls from the rota-rod apparatus was significantly higher in males injected by native TDP-43 than those of controls at 4 th week $(p<0.05)$. Grip test scores of females showed no difference between the control and experimental groups. In contrast, scores of the males in the experimental groups were significantly lower $(\mathrm{p}<0.01)$ than those of controls. MWM results displayed no significant difference between control and experimental groups in terms of latency to reach the platform and time spent in the target quadrant.

Conclusion: Transduction of animals with native or NLSdeleted TDP43 gene sequences results in gender-biased changes in motor functioning and disease onset without cognitive impairments. This relatively low cost, novel model might be helpful in testing therapeutic approaches in motor neuron diseases.

This study is supported by TÜBİTAK-Grant \#116S408.

Keywords: motor function, gender difference, TDP43, AAV, transduction

\section{PP9-004}

Corticospinal motor neuron specific transduction of the TARDBP gene via viral vectors

Furkan İlhan $^{1}$, Elif Polat Çorumlu ${ }^{1}$, İlkim Büyükgüdük ${ }^{1}$,

Handan Açelya Kapkaç ${ }^{2}$, Muhittin Arslanyolu²,

Emel Ulupinar

'Department of Interdisciplinary Neuroscience, Eskişehir Osmangazi University, Eskişehir, Turkey; ${ }^{2}$ Department of Molecular Biology, Eskişehir Technical University, Eskişehir, Turkey; ${ }^{3}$ Department of Anatomy, Faculty of Medicine, Eskişehir Osmangazi University, Eskişehir, Turkey

Objective: Recent studies show that mutations and overexpression of the TARDBP gene causes pathological aggregates and inclusion bodies in neurons. This is one of the underlying causes of the progressive neuronal degeneration and apoptosis seen in motor neuron diseases. In this study, we investigated the morphological changes in the rat motor cortex after transduction of neurons by viral vectors expressing either full-length
hTDP-43 or nuclear localization sequence-deleted hTDP-43 (hTDP-43- $\Delta$ NLS).

Methods: Experimental (hTDP-43 and hTDP-43- $\Delta$ NLS) and control (SF injected) groups of 4 week-old Sprague-Dawley rats ( $\mathrm{n}=6$, for each group) received intravenous injection of viral vectors (AAV2-9 serotype, 1.77 x1012 vg/kg) designed under the control of a specific promoter (ubiquitin carboxyl-terminal hydrolase-L1). After intracardiac perfusion at postnatal day 80 , serial frozen sections from the motor cortex were selected by using systematic random sampling approach. Neuronal profiles were stained by NeuN immunohistochemistry; whereas corticospinal motor neurons (CSMN) and callosal projection neurons (CPN) were labeled by Ctip-2 and Satb2 antibodies, respectively. Mean number of neurons in different cortical layers were counted, compared by using statistical methods.

Results: ANOVA results showed that mean number of total and CSMN were significantly $(\mathrm{p}<0.05$ and $\mathrm{p}<0.001)$ lower in animals transduced with full length hTDP-43, while no significant difference was observed in CPN. On the other hand, transduction with hTDP-43- $\Delta$ NLS caused no significant alteration in the number of CSMN or CPN in comparison to controls.

Conclusion: Our data revealed that overexpression of fulllength hTDP-43 induced more profound motor neuron degeneration than those of NLS-deleted hTDP-43. Designing of viral vectors with cell type specific promoters allowed us to transfer the genes into a specific region and specific subgroups of neurons in the motor cortex. This transduction method might be instrumental for targeting other cell types for generating animal models of neurodegenerative diseases in future.

This study is supported by TÜBİTAK-Grant \#116S408.

Keywords: corticospinal motor neuron, callosal projection neuron, neurodegenarative diseases, AAV2-9, viral vector

\section{PP9-005}

Using Xbox 360 kinect games to improve physical functions in multiple sclerosis

Simay Atıcı, Selen Gür Özmen

Department of Neuroscience, Graduate School of Health Sciences Bahçeşehir University, Istanbul, Turkey

Objective: Lately, using virtual reality games for rehabilitation of various diseases have been more popular all around the world. The aim of the study is to improve the physical skills of multiple sclerosis (MS) patients by playing Xbox 360 Kinect games.

Methods: The sample of the study consisted of patients diagnosed with Relapsing Remitting MS and Primary Progressive MS, who are treated in a private clinic in Istanbul. Berg Balance Test, Tandem Stance Test, Single Leg Stance Test, the Timed Up \& Go Test, the Activity-Specific Balance Scale, Fatigue Severity Scale, Fatigue Impact Scale, Quality of Life Assessment, Visual Analogue Scale, International Physical 
Activity Questionnaire were evaluated before the intervention of Xbox 360 Kinect games. Patients played the previously determined Xbox 360 Kinect games, an hour twice a week for 5 weeks. After 5 weeks, same physical parameters were evaluated again and pre and post evaluations were compared.

Results: An improvement in the Berg Balance Test scores of whole patients was observed both in pre and post evaluations. Among most of patients an enhancement observed in: Single Leg Stand Test with closed eyes position both right and left side, Timed Up\&Go Test, Quality of Life scores and Physical Activity Questionnaire. However no improvement was observed in Tandem Stand Test, Fatigue Impact Scale, Fatigue Severity Scale and Visual Analog Scale.

Conclusion: Virtual reality is gradually integrated as an additional method to medical rehabilitation all over the world. It can be used as a complementary and motivating method of both cognitive and physical rehabilitation for patients suffering from various neurological diseases. Randomized prospective studies in larger patient series should be done to verify our results.

Keywords: virtual reality rehabilitation, Xbox 360 kinect, multiple sclerosis

\section{PP9-006}

Effects of estrogen and progesterone on release of the neurogenic inflammatory neuropeptides from isolated rat trigeminal ganglia and cranial dura mater

Ayhan Çetinkaya ${ }^{1}$, Erkan Kılınç ${ }^{1}$, Çă̆rı Çamsarı ${ }^{2}$, Muhammed Nur Öğün ${ }^{3}$

'Department of Physiology, Bolu Abant Izzet Baysal University, Bolu, Turkey; ${ }^{2}$ Department of IFTDA Research Center, Bolu Abant izzet Baysal University, Bolu, Turkey; ${ }^{3}$ Department of Neurology, Faculty of Medicine, Bolu Abant Izzet Baysal University, Bolu, Turkey

Objective: Meningeal neurogenic inflammation caused by calcitonin gene-related peptide (CGRP) and substance P (SP) release from trigeminovascular system plays key role in migraine pathogenesis. Prevalence of migraine is 3 -folds higher in women than in men, and it is considered related to levels of estrogen and progesterone. But effects of estrogen and progesterone on CGRP and SP release from trigeminovascular system aren't known. We explored effects of estrogen and progesterone on CGRP and SP release from isolated rat trigeminal ganglia and meningeal trigeminal nerve terminals.

Methods: Isolated trigeminal ganglia and hemiskull preparations were prepared from adult male Wistar rats. Ganglia and hemiskull preparations were divided into $4 \operatorname{groups}(n=6)$, respectively, as follows: control, estrogen, progesterone, and estrogen+progesterone. Hemiskull preparations were incubated with $0.1 \%$ ethanol in artificial cerebrospinal-fluid for control group, $1 \mu \mathrm{M} 17 \beta$-estradiol for estrogen group, $1 \mu \mathrm{M}$ progesterone for progesterone group, and 17,-estradiol+progesterone for estrogen+progesterone group for $15 \mathrm{~min}$. Groups including isolated trigeminal ganglia were incubated with the same solutions and concentrations but for $5 \mathrm{~min}$. CGRP and $\mathrm{SP}$ contents in supernatants were measured using ELISA. Data were compared by one-way ANOVA.

Results: In hemiskull preparations, while progesterone decreased SP release $(\mathrm{p}=0.04)$, none of $17 \beta$-estradiol, progesterone and their combination changed CGRP or SP release ( $>0.05)$. In ganglia preparations, while $17 \beta$-estradiol reduced CGRP release $(\mathrm{p}=0.046)$, progesterone increased CGRP release $(\mathrm{p}=0.027)$, and combination of $17 \beta$-estradiol and progesterone didn't change CGRP release, but none of $17 \beta$-estradiol, progesterone and their combination changed SP release $(\mathrm{p}>0.05)$.

Conclusion: Progesterone alleviates neurogenic inflammation in meninges through inhibition of peripheral SP release, but it exacerbates by incuding CGRP release in trigeminal ganglia. Estrogen prevents neurogenic inflammation by reducing basal and progesteron-induced CGRP release in origin site of CGRP expression without affecting its peripheral release. Therefore, while estrogen has therapeutic effects, progesterone has dual effects on CGRP and SP release in different sites related to migraine pain.

Grant-number: 2017.08.02.1258(BAIBU-BAP).

Keywords: estrogen, progesterone, migraine, neurogenic inflammation, trigeminovascular system

\section{PP9-007}

Neurorehabilitation with parietal or frontal rTMS in primary progressive aphasia: two case reports

\section{Ece Zeynep Karakulak ${ }^{1}$, Lütfü Hanoğlu ${ }^{2}$}

'Department of Neuroscience, Graduate School of Health Sciences, Istanbul Medipol University, Istanbul, Turkey; ' Department of Neurology, Faculty of Medicine, Istanbul Medipol University, Istanbul, Turkey

Objective: Primary progressive aphasia (PPA) is observed accompanying neurodegenerative dementias. It is characterised by language impairment due to cortical network deformation. PPA is evaluated in three subtypes: semantic, logopenic and agrammatic variants. Translational neuroscience studies develop effective protocols with neuromodulation methods, such as rehabilitation and repetitive transcranial magnetic stimulation (rTMS), in order to slow down the progression and restore impaired functions. The language network is located in the frontotemporal and parietal areas and language functions can be improved with rTMS stimulation. In this clinical and retrospective case reports, it is aimed to investigate the effectiveness of rTMS combined with cognitive rehabilitation for PPA.

Methods: Cognitive rehabilitation and rTMS combination administered to two PPA patients after informed consent. The first case was an agrammatic PPA with speech apraxia and difficulty in grammatical structure. The second case was a logopenic variant PPA patient with errors in spontaneous speech, repeating failure and naming impairment. rTMS (20 Hz.) was applied on dorsolateral prefrontal cortex (DLPFC) of the first case and left inferior parietal region in the second case throughout 10 sessions with a 2 days interval after the 5 th ses- 
sion. Along with rTMS, rehabilitation sessions conducted in order to improve cognition and language functions. Before and after all intervention procedure, language functions were evaluated via phonemic and semantic fluency, and Boston Naming Test (BNT).

Results: Fluency test scores of the first case did not change after rTMS and rehabilitation, while spontaneous and phonemic cued naming scores increased in the BNT test. In the second case, both fluency tests and BNT scores increased.

Conclusion: Combined with rehabilitation, rTMS is one of the potential treatment approaches for PPA. In this study, promising results of stimulation on two regions of the language network are shown in PPA varants. Extensive research with neuroimaging methods for all three types of PPA should be encouraged.

Keywords: neurorehabilitation, primary progressive aphasia, rTMS

\section{PP9-010}

Essential muscle groups affected in cerebral palsy: systematic review

Asrın Nalbant, Özden Bedre

Department of Anatomy, Faculty of Medicine, University of Bakırçay, Izmir, Turkey

Objective: Cerebral palsy (CP) is a neurodevelopmental disorder of a nonprogressive central nervous system lesion. The aim of this study is to define the main muscle groups affected by cerebral palsy, to summarize the changes detected in the clinic and to determine the muscle or muscle groups that require detailed research.

Methods: In this systematic review, 495 studies published between 2000-2020 were analyzed. It has been searched using the keywords "cerebral palsy muscle contracture", "cerebral palsy muscle spasticity" and "cerebral palsy muscle strength" in Pubmed and Google Scholar search engines. Although the number of articles examined in the study was 495,73 studies that fit the limitations were included. Less studied or muscles that do not yet have sufficient data on the disease have been identified.

Results: Main muscle groups affected in cerebral palsy are leg muscles and ischiocrural muscles. The medial side of the gastrocnemius muscle is shorter in children with CP. There is a significant difference in terms of soleus muscle volume between people with CP and normal individuals. In the contracture of the hamstring muscle, squatting is remarkable. A positive relationship was found between age and knee flexor spasticity in children with CP. In children with CP; studies on the involvement of the gluteal muscles and external rotator muscles as well as the fibular muscles have been found to be insufficient.

Conclusion: Noticing the affected muscle structures in a timely manner in cases with $\mathrm{CP}$ allows some functions to be restored. In this way, possible problems can be prevented. Although the muscle structures of children with CP are examined separately; there are few studies examining the lower extremity alignment as a whole. With the effect of the hip muscles, the compensatory mechanism may affect the thigh, leg and foot muscles. Therefore, more comprehensive studies are needed.

Keywords: cerebral palsy, muscle strength, neurodevelopmental disorder, spasticity

\section{PP9-011}

Comparison of rotation behaviour of Wistar and genetic absence epilepsy rats injected with 6-hydroxydopamine

Aylin Toplu, Melis Yavuz, Yekta Çulpan, Zehra Nur Turgan, Rezzan Gülhan, Medine Gülçebi, Filiz Onat

Department of Medical Pharmacology, Faculty of Medicine, Marmara University, Istanbul, Turkey

Objective: 6-Hydroxydopamine (6-OHDA) is a toxic agent for the nigro-striatal dopaminergic neurons and it is used in modeling Parkinson's disease in rats (1). It is aimed to compare in genetic absence epilepsy rats (GAERS) and Wistar rats from Strasbourg, the apomorphine-induced rotation behavior, which is one of the indicators of dopaminergic damage of 6OHDA toxicity.

Methods: In this study, 30 days old Wistar and GAERS animals were divided into two groups. 6-OHDA $(8 \mu \mathrm{g}$ dose and $4 \mu \mathrm{L} / 4$ minutes) was injected by stereotaxic surgery into the medial forebrain bundle (MFB) (AP: -1.4; ML: 1.6; V: $7.1 \mathrm{~mm}$; single injection) of GAERS-MFB group ( $\mathrm{n}=8)$, into the striatum (AP:0.5/+0.5; ML:3.0; V: $5.0 \mathrm{~mm}$; double injections) of GAERSStriatum group $(\mathrm{n}=5)$, as well as into the MFB (AP:-1.4; ML: 1.6; $\mathrm{V}: 7.1 \mathrm{~mm}$; single injection) of Wistar-MFB group ( $\mathrm{n}=4)$ and the striatum (AP: -0.5/+0.5; ML: 3.0; V: $5.0 \mathrm{~mm}$; double injections) of Wistar-Striatum ( $\mathrm{n}=2)$ group. After 21 days, all animals were injected with apomorphine $(0.05 \mathrm{mg} / \mathrm{kg}$, subcutan). After apomorphine administration, right and left rotation (3600) of the animals were recorded for 30 minutes. Data were expressed as mean \pm standart error. One-way ANOVA and Tukey post-hoc test were used $(\mathrm{p}<0.05$ was considered significant).

Results: The mean number of GAERS-MFB rotations was $6.56 \pm 0.05$ per minute, whereas the GAERS-Striatum was $6.19 \pm 1.72$ per minute. While the number of Wistar-MFB rotations was $4.74 \pm 2.33$ per minute, the number of Wistar-Striatum rotations was $3.27 \pm 1.40$ per minute. There was no statistically significant difference between the groups.

Conclusion: Although the findings show there is no difference between the groups, it is aimed to continue the study by increasing the number because of the numbers of groups are limited in our study. The study is supported by TÜBİTAK (218S653).

Keywords: 6-OHDA toxicity, wistar, GAERS, rotation 
PP10

Other (Neuroscience Education, Neuroethics, Neurophysiology, Neuroeconomics etc.)

\section{PP10-002}

Examining the distribution of postgraduate theses in the field of neurosciences in Turkey

Serdar Saritas ${ }^{1}$, Sultan Tarlaci ${ }^{2}$

'Department of Surgical Nursing, Inönü University, Malatya, Turkey; ${ }^{2}$ Department of Neurology, Faculty of Medicine, Üsküdar University, Istanbul, Turkey

Objective: In recent years, the interest in neurosciences has increased both in Turkey and across the world, whereby postgraduate programs in neurosciences have been offered at several universities. In this field, which is referred to as "neuroscience" in English but referred to as "sinirbilim" and "nörobilim" in Turkish.

Methods: This is a descriptive study. For the research, the Databases of National Thesis Center of the Council of Higher Education website (https://tez.yok.gov.tr) has been visited for July 20-23,2020; and all departments in Turkey have been examined using the detail search link in this website. As a result, a total of nine departments were determined, including Neurosciences (Sinirbilim), Neurosciences (Nörobilim), Neurological Sciences, Clinical Neurosciences, Interdisciplinary Neurosciences, Neuromarketing, Basic Neurological Sciences, Clinical Neurological Sciences and Psychiatry, and Neuroelectrophysiology, which were considered to be associated with neurosciences. Therefore, theses in these departments were included in the scope of study.

Results: Accordingly, a total of 502 theses were produced between 1998 and 2020 in Turkey, of which 388 (77.13\%) were master's thesis and 100 (19.99\%) were doctoral theses. Although 14 theses were presented as a thesis for specialty in Neurology, they were categorized incorrectly in the field of neurosciences. Considering the distribution of theses based on departments; $316(62.88 \%)$ of the theses were produced in the Department of Neurosciences (Sinirbilim), 81 (16.11\%) in the Department of Neurosciences (Nörobilim), and 50 (9.95\%) in the Department of Neurological Sciences.

Conclusion: The number of universities offering postgraduate education in neurosciences has been gradually increasing in Turkey. Although some universities have assigned departments specific to this field, they have no theses registered in the thesis center yet. Thanks to the high number of neuroscience programs opened at foundation universities in recent years, an increasing number of theses have been produced in this field, whereby neuroscience has become more widely known in Turkey.

Keywords: postgraduate theses, neuroscience, university

\section{PP10-003}

The importance of JOVE in neuroscience research

Gürol Atar

Interdisciplinary Neurosciences, Eskişehir Osmangazi University, Eskişehir, Turkey

Objective: The importance of research and education opportunities offered by JOVE, the world's first and only video platform with peer-reviewed where the articles are presented visually, in neuroscience research and education has been examined. The research aims to evaluate the usage rate of JOVE in Turkey and the world in the field of neuroscience.

Methods: For the research, some information about usage numbers was requested from JOVE. Some observations and some comparisons were made based on these data.

Results: Based on the data obtained from JOVE, the articles in the field of Neuroscience ranked fourth after the Medicine, Biology, and Engineering departments in the viewing ranking. Top 10 universities in the field of neuroscience using JOVE in the last five years: (1) Johns Hopkins University, (2) Columbia University, (3) Stanford University, (4) The University of Tokyo, (5) Washington University- St. Louis, (6) University of California- San Diego, (7) University College London, (8) Harvard University, (9) University of Michigan: Ann Arbor, (10) Yale University. In Turkey, thirty universities have a JOVE license, but these universities are located at a low rank in the utilization rate of JOVE.In the last ten years, only twelve articles (only one neuroscience article) have been published from Turkey.

Conclusion: It has been observed that universities that publish in high prestigious journals and have high-level research outputs are at the top in the use of JOVE. Although the universities named above have very high research funds and extensive laboratory facilities, it seems that they have made the use of JOVE a part of their research and education. It is thought that increasing the use of JOVE, which saves time for researchers and students, and provides detailed opportunities for new research techniques, both in research and as a part of education, will have a positive effect on the research outputs that will emerge.

Keywords: JOVE, neuroscience, research

\section{PP10-004}

Neuro-law as an interdisciplinary law and the reflections of developments in neurotechnology to the human rights field

Tamer Soysal

Ministry of Justice, Ankara, Turkey

Objective: The development of neuro technologies and neuroscience also requires the establishment of a legal framework on the subject. Neuroscience examines the brain processes underlying human behavior; law also deals with the regulation 
of human behavior. This intersection area is tried to be organized and categorized in the field of expertise called "neurolaw". Neurolaw is a field of interdisciplinary study that explores the effects of discoveries in neuroscience on legal rules and standards. Neurolaw studies the effect of neuroscientific evidence on law and legal cases. Neuro-science techniques, which include the researches for the causes and prevention of crime, are called neuro-criminology. Neuro-criminology searches for a correlation between the structure and features of the brain and criminal behavior, and it underlies behaviors such as criminal responsibility and revenge. New information about the functioning of the brain, testing the reliability of witnesses, or new brain imaging techniques can assist in criminal justice, the determination of the limits of responsibility and the rehabilitation of criminals. On the other hand, incredibly rapid developments in the field of neurotechnology require a new concept and perspective on human rights. In our paper, the area of law called neuro-law will be examined, and then human rights risks that may arise due to neurotechnology will be discussed.

Methods: Literature Review and Participation in International Meetings in this Field.

Results: The emergence of the rules of neuro-law as an important field that will be shaped by the contribution of scientists working in this field together with lawyers.

Conclusion: The implications of neurotechnology in the field of law will begin to be discussed more and more, and neuro-law will emerge more clearly as a separate field of law.

Keywords: 1- neurotechnogy, 2- law, 3- neuro-law, 4- neurocriminology, 5- human rights

\section{PP10-005}

\section{Creating new models in neuroanatomy training by using 3D printer}

Tuncay Veysel Peker ${ }^{1}, \underline{\text { Ece Alim}}^{2}$

${ }^{1}$ Department of Anatomy, School of Medicine, Gazi University, Ankara, Turkey; ${ }^{2}$ Department of Anatomy, School of Medicine, Gazi University, Ankara, Turkey \& Neuroscience and Neurotechnology Center of Excellence (NÖROM), Ankara, Turkey

Objective: Although the brainstem is dimensionally small, it contains all the cranial nerve nuclei and their nerve fibers except the optic and the olfactory nerve. Learning them with traditional medical education is difficult. Knowledge and visual materials related to the brainstem in neuroanatomy books/ atlases are static and limited with two dimensions. Our aim in this study isn't to analyze morphometric analysis but to create a model to show cranial nerves, cranial nerve nuclei, descending-ascending pathways and their 3-dimensional spatial relationships with each other.

Methods: All parts of the brainstem was separately modeled. $3 \mathrm{D}$ modeling and animation software was used for modeling. The external surface model and the internal structures of the brainstem were printed out separately in the $3 \mathrm{D}$ printer. Also, the models have transferred to Unity Game Engine and
Vuforia software for Augmented Reality applications. The model was made ready with silicone mold and polyester resin.

Results: Models and education tools that shows the brainstem nuclei and their internal anatomic structures are still insufficient in the world. This study is unique with regard to this feature in the world. When the model is being kept in the hand, all projection tracts, locations of the cranial nuclei and 3D spatial anatomical relationships are visible and clear. The $3 \mathrm{D}$ model of the brainstem is 15 times larger than the actual human size. The reconstructed model can be rotated $360^{\circ}$ in all axes. It is also possible to walk around the internal structures by inserting a virtual camera into the model.

Conclusion: The model created in the study will make it easier to learn the brain stem, which is difficult to learn in classical medical education. It will also allow surgeons to make easier microsurgical interventions to the brainstem. In future studies, augmented reality, artificial intelligence or electronic components will be added to these models.

Keywords: 3D modeling, augmented reality, 3D printing, brainstem, neuroanatomy

\section{PP10-006}

\section{Did you know the nucleus ambiguus has a parasympathetic function?}

Mehmet Ülkir, Helin Yücedağg, Burcu Erçakmak Güneş

Department of Anatomy, Faculty of Medicine, Hacettepe University, Ankara, Turkey

Objective: Nucleus ambiguus is located lateral to lemniscus medialis, dorsal to inferior olivary nucleus. It lies between nucleus of hypoglossal nerve and inferior olivary nucleus at medulla oblongata. Embryologically, cells having the mature neurons seen rostrally in the nucleus during the 8.5-9 weeks and later form the whole nucleus at 12.5 weeks. Nucleus ambiguus is composed of the dorsal branchial motor unit and ventral general visceral unit. Dorsal unit is divided into compact, semicompact and loose compact regions. Ventral unit contains pregnaglionic neurons that are innervating the heart and thoracic structures. The area of the medulla oblongata which the nucleus ambiguus lies, is supplied by the vertebrobasilar system. When the literature is reviwed, there are few studies on parasympathetic heart function.Aim of this review is reveal to anatomy, heart and parasympathetic functions, clinical implications of nucleus ambiguus

Methods: The key words are writed PUBMED as nucleus ambiguus, parasympathetic, heart innervation and literature review is performed.

Results: Afferents of the nucleus ambiguus are mostly comes from the nuclei of solitary tract. While this nucleus receives sensory information from baroreceptors, the majority of occurred parasympathetic responses are generated by the nucleus ambiguus. These emerging general visceral efferent fibers inhibit signals to the SA node and reduce heart rate. These parasympathetic effects occur as a result of the depolar- 
ization of bradykinin, $G$ protein-binding estrogen and urotensin 2 receptors of the nucleus. In conditions such as Wallenberg, Avellis and Tapia syndromes the nucleus ambiguus may be affected and lead to varity of clinical symptoms.

Conclusion: Knowing the anatomy, functions and clinical relations of the nucleus ambiguus are important for clinical evaluations and procedures.

Keywords: nucleus ambiguus, neuroanatomy, parasympathetic

\section{PP10-007}

The investigation of eye movement patterns in human robot interaction

Cengiz Acartürk, Elif Esmer, Gamze Eşdur, Gizem Özen METU Cognitive Science, Ankara, Turkey

Objective: Gaze contact is of major importance in social interaction. For the research domain of Human Robot Interaction, which conceives communication strategies in human-human interaction as a modeling framework, gaze contact is the key for natural and efficient communication. In this study, a comparison of the interaction between human-human and humanrobot interlocutors was conducted by investigating eye move- ment patterns, within the context of a mock-up job interview scenario. In two scenarios, one of which is to give answers to a human and the other is to give answers to a robot, eye movement patterns were investigated.

Methods: The study is composed of three experiments. In the first experiment, the participants answered a set of question asked by an expert and their eye movements were recorded during the course of answering. The eye movement data were used for obtaining fixation location, duration and order, thus total gaze aversion and gaze contact ratios. In the second experiment, the same setting was employed to record the eye movements of the interviewers. In the third experiment, the eye movements of the robot interviewer were designed by the data obtained in the second experiment.

Results: The findings reveal that human-human implementation reveals similar methods in the human-robot interaction setting.

Conclusion: Accordingly, the findings reveal that humanhuman data can be used as a basis for designing human-robot interaction.

Keywords: human-robot interaction, human-human inteaction, eye tracking, gaze contact, gaze aversion 


\section{anatomy}

\section{8th Turkish Neuroscience Congress 6-9 November 2020, Ankara, Turkey}

\begin{tabular}{|c|c|}
\hline \multicolumn{2}{|l|}{ A } \\
\hline Abidin İ & PP3-001 \\
\hline Abidin SA & PP3-001 \\
\hline Acar B & SS1-C01, SS1-C04, SS3-A01, SS3-A03 \\
\hline Acartürk C & PS7-3, PP10-007 \\
\hline Adams M & C-02, SS1-A01 \\
\hline Adams MM & PP1-006, PP5-011 \\
\hline Ademoğlu A & PP8-002, PP8-009 \\
\hline Adıgüzel E & PS13-1 \\
\hline Akarca Dizakar Ö & SS1-A06 \\
\hline Akcay G & SS3-C04, PP3-002 \\
\hline Akdal G & SS3-B03, PP5-014 \\
\hline Akdemir Aktaş H & PP5-048 \\
\hline Akkoç T & SS3-A06 \\
\hline Akkoyun M & SS3-B03, PP5-014 \\
\hline Akman Ö & PS9-2, SS2-B03 \\
\hline Akmeşe C & PP5-005 \\
\hline Aksan Kurnaz I & PS2-2 \\
\hline Aksu A & SS2-B02 \\
\hline Aktert Ayar D & SS3-B06 \\
\hline Aktürk T & PS6-3, PS10-4, SS2-C01 \\
\hline Akyan S & SS3-C07 \\
\hline Akyüz S & PP5-029 \\
\hline Aladağ Z & PS2-2 \\
\hline Alaşhan D & PS15-2 \\
\hline Albayrak ÖF & PP5-008 \\
\hline Albright $\mathrm{T}$ & $\mathrm{C}-07$ \\
\hline Alim E & SS1-A06, SS3-C03, PP10-005 \\
\hline Alkın T & PP1-001 \\
\hline Alp Mİ & PP1-002 \\
\hline Alptekin K & SS3-B04 \\
\hline Altınok S & PP8-003 \\
\hline Altun E & PP5-007, PP5-045 \\
\hline Altuntaş Ö & SS3-A02 \\
\hline Andaç S & PP5-005 \\
\hline Ann Hirsch J & SS3-C05 \\
\hline Anwander A & PS5-1 \\
\hline Apaydın N & PP8-003 \\
\hline $\operatorname{Ar} 1 \mathrm{~F}$ & PP5-016 \\
\hline Arıca Akkök E & SS1-B01 \\
\hline Arıcıoğlu F & SS2-B03 \\
\hline Arıkan O & PP7-006 \\
\hline Arkan S & SS2-C02 \\
\hline Armspach JP & PP8-004 \\
\hline Arslan Ergül A & PP1-006 \\
\hline Arslanyolu M & PP9-002, РP9-004 \\
\hline Aslan M & SS3-C04 \\
\hline Aşkun CS & PS3-4 \\
\hline Atalay Ö & PP5-009 \\
\hline Atar G & SS1-A07, PP1-008, PP10-003 \\
\hline
\end{tabular}

Atay E
Atayolu R
Ateş G
Ateş M
Ateş N
Ateş Öz E
Atıcı S
Atum M
Ay U
Ayas H
Aydın AE
Aydın B
Aydınlar İ
Aydoğan HÖ
Ayhan I
Aykaç C
Aykan S
Aypar B
Ayten UE
Ayyldız N
Ayyubova G
B
Babal YK
Badakul AN
Bahadır Varol A
Bahçelioğlu M
Baka M
Baksak B
Bal NB
Balcı F
Balkan B
Balkıs N
Baran Z
Barkana DE
Baskak B
Başar Eroğlu C
Başkak B
Bayirli M
Bayrak SB
Bayramera Ö M
Bayraktar S
Bayram A
Berberoğlu Z
Berger M
Beyer F

\author{
SS2-C01 \\ PP1-002 \\ SS2-B02 \\ SS2-C05 \\ SS2-B03, SS2-C06 \\ PP1-002 \\ PP9-005 \\ SS1-C06 \\ SS1-B06, SS1-C07, SS3-A02 \\ PP5-024 \\ SS3-A07 \\ SS1-B04 \\ SS2-C07 \\ PP1-006 \\ PS15-2 \\ PP1-001 \\ PS14-3, PP5-008 \\ SS3-B07 \\ PS2-2 \\ PS5-1, PS5-2, PS5-3, PS5-4 \\ PP1-005
}

PP7-002
PP5-027
PP5-002
SS1-A06, SS3-C03
PP2-001
SS3-B02, PP8-003
SS2-A05
SS2-C02
SS3-B07
SS2-B02
SS3-B02
PP5-026, PP5-031
SS2-A05
C-03, PS6-2, PS6-4
SS2-C03
SS3-B05
PP8-009
PP5-045
PS10-2
PS6-4, SS1-C01, SS1-C04, SS1-C07,
SS3-A01, SS3-A02, PP8-002
PP5-008
PP9-010
SS2-B06
PP5-018
SS3-B04
PS5-1 


\begin{tabular}{|c|c|c|c|}
\hline Bienert $\mathrm{T}$ & PP8-004 & Danışman B & PP3-002 \\
\hline Bilgiç B & SS1-C07 & Degiorgis L & PP8-004 \\
\hline Bilgin MD & SS2-B07 & Delen K & PP6-002 \\
\hline Bilim P & PP2-001 & Demir EA & SS3-B05, PP5-017 \\
\hline Bilir Yıldız B & SS2-B07 & Demir İ & SS1-B06 \\
\hline Birdoğan A & SS3-B07 & Demir N & PP5-012 \\
\hline Biterge Süt B & PP1-011 & Demiralp T & PS6-4, SS1-B06, SS1-C01, SS1-C02, \\
\hline Bolay Belen H & C-09 & & SS1-C04, SS1-C07, SS3-A01, SS3-A02, \\
\hline Bora $\mathrm{E}$ & SS1-C05 & & SS3-A03, PP8-002 \\
\hline Boyac1 H & PS15-1, PP4-001, PP5-033, PP5-047 & Derin $\mathrm{N}$ & SS3-C04, PP3-002 \\
\hline Boyraz RK & SS2-B06 & Deveci E & SS2-B06 \\
\hline Bozdağ Pehlivan S & PS1-3 & Devlet Kilıçkap B & SS3-C06 \\
\hline Bozkurt BT & PP1-015 & Devrimci Özgüven H & SS3-B02, PP8-003 \\
\hline Bozkurt Girit Ö & SS2-B07 & Dilsiz P & PP1-002 \\
\hline \multirow[t]{2}{*}{ Büyükgüdük İ } & PP9-002, PP9-004 & Dinç D & PP5-013 \\
\hline & & Direk N & PP1-001 \\
\hline C-Ç & & Doerschner K & PS15-3 \\
\hline Can $\mathrm{H}$ & SS2-C07, SS3-A05 & Doğan H & SS3-B05 \\
\hline Candar E & PP1-010 & Doğan RB & PP8-010 \\
\hline Cangöz B & SS1-B02, SS3-B02 & Doğanay Erdoğan B & PP8-011 \\
\hline Canlı M & SS1-C04 & Dost D & PP7-003 \\
\hline Cansever ÖM & PP5-028 & Dönmez Çolakoğlu B & PP5-014 \\
\hline Cedden G & PP5-018 & Dövencioğlu DN & PS15-3 \\
\hline Ceran Ö & SS2-C01 & Duman F & SS3-A05 \\
\hline Ceylan D & SS1-C05 & Duran N & SS3-B05 \\
\hline Coşkun $M$ & SS3-A02 & Dursun İ & SS1-A05 \\
\hline Cowan NJ & SS1-C03 & Duyan YA & SS2-C02 \\
\hline Çağıl İnal Ş & PP5-037 & Düz ME & PP8-008 \\
\hline Çakır MP & PS7-2, SS2-C03, PP5-021 & Düzenli Öztürk S & PS14-4, SS1-B05 \\
\hline Çakmur R & PP5-014 & & \\
\hline Çalıkuşu FZ & PP5-042 & $\mathbf{F}$ & \\
\hline Çalışoğlu P & PS6-3, SS2-C01 & $\mathrm{E}$ & \\
\hline Çamsarı Ç & PP9-006 & Ebru Köse F & SS3-B02 \\
\hline Çapar A & PS2-2 & Eken A & SS2-A05, PP5-018 \\
\hline Çarçak N & PS9-3 & Ekin Sönmez & PP1-014 \\
\hline Çatak EN & PP8-001 & Ekinci B & PP1-001 \\
\hline Çelebisoy N & SS3-B06 & Ekinci DA & PS2-2 \\
\hline Çelik E & PS2-2, PP7-004 & Elibol B & SS1-A05, SS2-A04, PP5-002 \\
\hline Çelik K & SS3-B06, PP2-001 & Elmas HO & PP7-007 \\
\hline Çelik S & PP8-010 & Elsürer Ç & PP8-007 \\
\hline Çelik Z & PP5-048 & Engür D & SS1-A02 \\
\hline Çelikağ İ & PP8-003 & $\operatorname{Er} M N$ & PP5-007 \\
\hline Çetinkaya A & PP9-006 & Er N & PP5-045 \\
\hline Çetintulum Huyut B P & P6-001 & Er S & PP7-007 \\
\hline Çevik MN & PP5-027 & Ercan İ & SS1-A02 \\
\hline Çiçek GB & PP5-019 & Erçakmak Güneş B & PP10-006 \\
\hline \multirow[t]{2}{*}{ Çiçek M } & PS5-1, PS5-2, PS5-3, PS5-4, PP8-003, & Erdemir Kızıltan M & SS3-C01, PP5-042 \\
\hline & PP8-011 & Erden F & PP5-046 \\
\hline Çiflik Y & PP5-027 & Erdener E & PS4-1 \\
\hline Çiftçioğlu UM & SS3-C05 & Erdeniz B & SS1-C05 \\
\hline Çimen F & SS3-B05 & Eren Koçak E & PS4-1, PP5-002 \\
\hline Çimen Yetiş $S$ & PS2-2 & Ergen $M$ & SS2-C04 \\
\hline Çiyiltepe MM & PP5-024 & Ergenoğlu T & PP5-013 \\
\hline Çoban Çiftçi G & PS3-3 & Erkuş EC & PP7-005 \\
\hline Çolak B & SS2-A05 & Ermiş Ç & PP1-001 \\
\hline Çögen T & PP5-031 & Eroğlu Ada F & SS2-C01 \\
\hline Çukur T & PS8-3, PP7-004 & Ersoy Tunalı N & SS2-B05 \\
\hline \multirow[t]{2}{*}{ Çulpan Y } & SS2-B01, PP9-011 & Erten $\mathrm{F}$ & $\mathrm{SS} 3-\mathrm{C} 03$ \\
\hline & & Eryürek K & SS1-B06, SS1-C07, SS3-A03 \\
\hline $\mathbf{D}$ & & Esen EC & PP5-002 \\
\hline Dalbayrak B & PP1-014 & Esmer E & PP10-007 \\
\hline Dalkara T & C-06, PS4-1, PS13-2, PP5-002 & Eşdur G & PP10-007 \\
\hline
\end{tabular}


S164 18th Turkish Neuroscience Congress, 6-9 November 2020, Ankara, Turkey

F

Fang F

Fazlığulları Z

Ferikoğlu S

Fidanc1 A

Fide $\mathrm{E}$

Finsen B

Fortune ES

\section{G}

Garip G

Geduk S

Genç Ş

Göçücü K

Gökçay D

Gökçe E

Gökmen RC

Göktepe G

Gözen $\mathrm{O}$

Güçlü B

Gülbetekin E

Gülbol Duran G

Gülçebi İdriz Oğlu M

Gülçebi M

Gülekon INN

Güler B

Gülhan R

Gümüş H

Gümüşlü E

Günal Değirmendereli G

Gündüz A

Günenç Beşer C

Güneş E

Güntekin B

Gür E

Gür Özmen S

Gürbüz BT

Gürcan E

Gürsoy Özdemir Y

Gürvit H

Gürvit İH

Güvenç G

Güvendi G

H

Halıcı U

Hanalioğlu Ş

Hanoğlu L

Harı E

Harsan LA

Hoşgörler FU

Humo M

Huseynova S

Hünerli Gündüz D

\section{I-İ}

$\begin{array}{ll}\text { Ildız A } & \text { SS1-C05 } \\ \text { Ilgaz HB } & \text { PP4-001 } \\ \text { Ilgım Ardıç N } & \text { PP1-006 }\end{array}$

Ilgin R SS2-C05
Irak $M$

Işıkay İ

Iş1 Kurnaz

Işıldar HK

İder YZ

İlayda Aydınlı F

İlhan ÇF

İlhan $\mathrm{F}$

İnal Ç

İşleten Hoşoğlu M

K

Kadıhasanoğlu D

Kafalıgönül H

Kalaycıŏlu C

Kale EH

Kandemir B

Kandiş $\mathrm{S}$

Kanit L

Kantar D

Kapkaç HA

Kaptan Z

Karabulut AK

Karadenizli Taşkın S

Karaduman A

Karagöz M

Karagün E

Karakaş S

Karakılıç A

Karakulak EZ

Karamahmutoğlu T

Karataş Kurşun H

Karataş M

Karatay O

Karlı Oğuz K

Karoğlu Eravşar ET

Karslı B

Karson A

Kartelli F

Kasapoğlu B

Kaşıkçı I

Kaval Oğuz E

Kaya U

Kayasandık CB

Kerman BE

Keser A

Keser H

Keskin S

Kılınç E

Kinay D

Kınıklığlu M

Kırbaş OK

Kıyı İ

Kıymaz KZ

Kızıl Ç

Kızılateş Evin G

Kızıldağ S

Kızılpınar SÇ
PS12-1, PS12-2, PS12-3, PP5-028

PS3-2

PP1-003

PP1-002

PS8-2

PS2-2

PP9-002

PP9-004

PP5-008

PP1-014

SS2-A03

PS13-6, PP5-003, PP5-011, PP5-029,

PP8-001

PS14-1, SS3-C07

PS5-1, PS5-3, PP8-003

PP1-003

SS2-C05

PS13-5, SS3-B07

PP5-045

PP9-002, PP9-004

SS3-B01

PP8-007

SS2-C06

PP5-011

PP5-017

SS2-C06

PS6-1

SS2-C05

PP9-007

SS2-B01

PP5-002

PP8-004

SS1-C04

PS3-3

SS1-A01, PP5-011

PS12-1, PS12-3

SS2-B03, SS2-C02

SS3-B04

PP1-015

PP5-026, PP5-031

SS3-A06

PP5-029

PS10-3

PS2-2

SS3-B07

PP3-001

SS3-A06

PP9-006

SS3-A02

PP5-033

PP1-015

PS6-3, SS3-B04, PP5-015, PP5-023

PP3-002

PS2-3

SS1-C01, SS1-C04, SS1-C07, SS3-A01

SS2-C05

SS2-A05 


\begin{tabular}{|c|c|c|c|}
\hline Kiser Ç & SS1-A02 & Önal C & PP1-011 \\
\hline Kocatürk M & PS11-4 & Önal D & PP5-009 \\
\hline Koç B & SS2-C05 & Önder B & PP1-001 \\
\hline Koç Yılmaz Ş & PP5-010 & Öner Ö & PS5-1, PS5-2, PS5-3 \\
\hline Koçoğlu K & SS3-B03, PP5-014 & Öz BK & PP5-031 \\
\hline Koenderink J & PS15-3 & Özaydın Aksun Z & SS1-B01 \\
\hline Konaç E & SS1-A06, SS3-C03 & Özbek Y & SS3-B04, PP5-015 \\
\hline Konyalı A & PP5-003 & Özdemir Ş & SS1-B07 \\
\hline Koylu EO & SS3-B07 & Özden Şener H & SS1-B01 \\
\hline Köse H & PP5-031 & Özel Kızıl ET & SS3-B02 \\
\hline Kumral A & SS1-A02 & Özen G & PS7-4, PP10-007 \\
\hline Kumral E & SS3-B06 & Özen N & PP5-034 \\
\hline Kumru H & SS3-C01 & Özerdem A & SS1-C05 \\
\hline Kurada HZ & SS1-B01 & Özgen E & PS1-1, PP5-010 \\
\hline Kurnaz I & PP1-014, PP7-002 & Özkan M & PP8-001 \\
\hline \multirow[t]{2}{*}{ Kurt E } & SS1-B06, SS1-C01, SS1-C04, SS1-C07, & Özkök E & SS2-B02 \\
\hline & $\mathrm{SS} 3-\mathrm{A} 01, \mathrm{SS} 3-\mathrm{A} 03$ & Özkurt TE & PP5-020 \\
\hline Kurt $\mathrm{H}$ & PP1-014 & Öztura İ & PP5-012, PP5-023 \\
\hline Kurt Vatandaşlar B & PS2-2 & Öztürk A & PP7-003 \\
\hline Kuruoğlu A & PP1-001 & Öztürk M & SS3-B07, PP8-007 \\
\hline Kuşman A & SS2-A05 & & \\
\hline Kuzay D & PP6-002 & $\mathbf{P}$ & \\
\hline Küçük İ & PP1-014 & Pamuk BŞ & PP2-001 \\
\hline \multirow[t]{2}{*}{ Küçük M } & PS6-2 & Parlatan CS & PP8-010 \\
\hline & & Paslı B & PP4-001 \\
\hline $\mathbf{L}$ & & Pavan A & PP5-029 \\
\hline Lamy J & PP8-004 & Paxinos G & SS1-A03 \\
\hline Lee $\mathrm{J}$ & $\mathrm{C}-05$ & Pehlivanoğlu B & PP5-009 \\
\hline \multirow{2}{*}{ Leventoğlu A } & SS3-A05 & Peker K & PP8-007 \\
\hline & & Peker TV & PP10-005 \\
\hline $\mathbf{M}$ & & Petekkaya E & SS3-B01 \\
\hline & & Polat Çorumlu E & PP9-002, PP9-004 \\
\hline Mançe Çalışır O & PS5-1, PS5-3 & Purutçuoğlu V PP7-005 & \\
\hline Martin L & PP1-008 & & \\
\hline Maviş İ & SS1-B07 & & \\
\hline Metaxas A & PP1-008 & S-Ş & \\
\hline Metiner MD & PP1-003 & Saçkesen İ & PP5-008 \\
\hline Mursalova Ünal S & SS2-B06 & Sadigi İ & PP1-005 \\
\hline Mut Aşkun M & PS3-2 & Sağır F & SS2-B05 \\
\hline Mutlu O & PP5-046 & Salman F & SS1-B02 \\
\hline \multirow[t]{2}{*}{ Mutlu P } & PP7-002 & Sarışık E & $\mathrm{SS} 1-\mathrm{C} 02$ \\
\hline & & Sarıtaş S & PP10-002 \\
\hline \multirow{2}{*}{$\mathbf{N}$} & & Sarper Kahveci M & SS2-C06 \\
\hline & & Sayar Akaslan D & SS2-A05 \\
\hline Nalbant A & PP9-010 & Saybaşılı H & PP1-014 \\
\hline Nalçacı E & PP5-008, PP5-016 & Saygin AP & PP7-007 \\
\hline Neşe H & PP8-002 & Schmiedt Fehr C & PS6-2 \\
\hline Newman SD & SS2-A02 & Seçkin $M$ & SS1-B06 \\
\hline Noblet $\mathrm{V}$ & PP8-004 & Semerci YC & PP5-026 \\
\hline & & Sirav B & PP6-002 \\
\hline O-Ö & & Sirmatel B & PP8-011 \\
\hline Oklun S & PS5-1 & Sokullu E & PS1-2 \\
\hline Oktay Y & PP1-001 & Somer O & PP5-019 \\
\hline Okurgan E & PP5-040 & Soncu Büyükişcan E & SS3-A03 \\
\hline Olcum M & SS1-A02 & Sourty M & PP8-004 \\
\hline Olkun S & PS5-2, PS5-3 & Soylu C & PS12-1, PS12-2, PS12-3 \\
\hline Onat F & PS9-1, SS2-B01, PP9-011 & Soysal T & PP10-004 \\
\hline Oruç S & PP6-002 & Soytürk H & PP1-011 \\
\hline Ozansoy M & PS2-1, SS1-A04 & Sönmez E & PP7-002 \\
\hline Ozansoy MB & SS1-A04 & Stenken D & PP5-027 \\
\hline Öğün MN & PP9-006 & Stoner G & PP8-001 \\
\hline Ölcener Ariburnu D & SS2-A07 & Şahbudak Y & PP5-013 \\
\hline
\end{tabular}


S166 18th Turkish Neuroscience Congress, 6-9 November 2020, Ankara, Turkey

$\begin{array}{ll}\text { Şahin F } & \text { PP1-015 } \\ \text { Şahin K } & \text { SS3-C03 } \\ \text { Şeker A } & \text { SS3-A06 } \\ \text { Şeker M } & \text { PP8-008 } \\ \text { Şekerdağ E } & \text { PS1-4 } \\ \text { Şengör NS } & \text { PS13-4,SS2-A04 } \\ \text { Şengül G } & \text { SS1-A03, PP1-010 } \\ \text { Şimşek H } & \text { PP8-011 }\end{array}$

$\mathrm{T}$

Tamer Ş

Tanriverdi D

Tanriverdi U

Tanseli Kaspar G

Tarlaci S

Taşkan E

Tatar I

Terzi MB

Terzioğlu Uşak Ş

Tetik Oktay M

Thygesen C

Tobias Sommer F

Tombul T

Topçugil B

Toplu A

Töreyin BU

Tumer C

Tuncer A

Turan G

Turan SN

Turgan Aşık ZN

Turgan $\mathrm{ZN}$

Tutuk $\mathrm{O}$

Tuzcu F

Tüfekci UK

Tülay EE

Türe U

Türkakın E

Türker RS

Türkoğlu R

Tüz Şaşik MU

Tüzün E

U-Ü

Uddin L

Ulaşoğlu Yıldız Ç

Ulupınar E

Ulusoy İ

Uran P

Urgen BM

Urhan Küçük M

Usal KA

Utkan T

Uyanık I

Uygun I

Uysal N

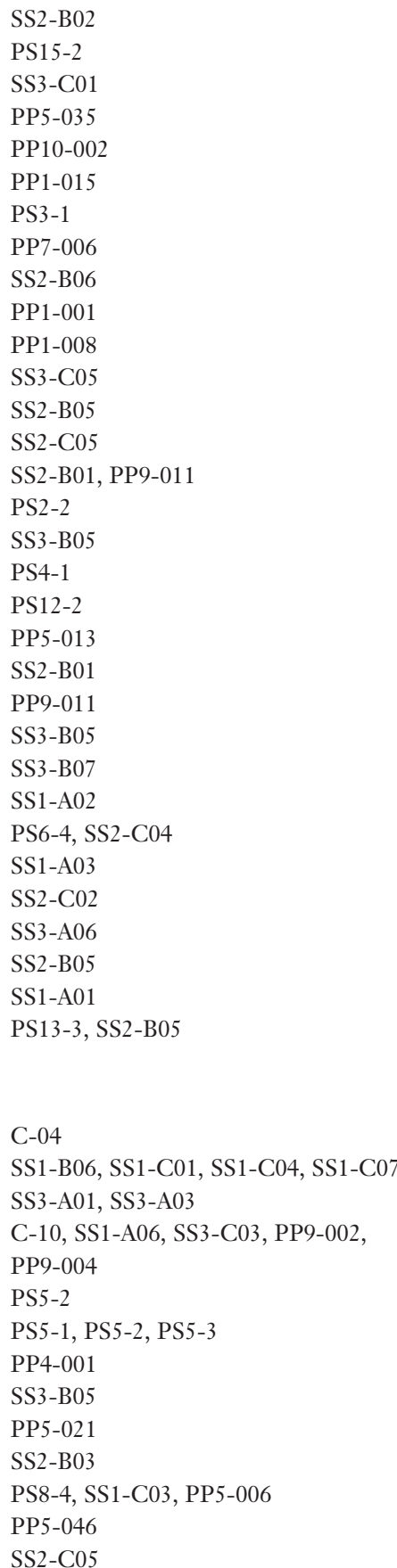

Uzunlar H

Uzunlar SH

Ülkir $M$

Ünal B

Ünal ÇT

Ünal G

Ünver Doğan N

Ürgen BA

Üstün R

Üstün S

Üzmez N

V

Van Doorn A PS15-3

Vardar B SS3-C06

Vargeloğlu S PP5-006

Vatandaşlar E PS2-2

Vatansever G PS5-2, PS5-4

Velioğlu HA PS10-3

Velioğlu SK PS9-4

Verim B SS1-C05

Villringer A

von Elverfeldt D PP8-004

W

Witte VA

Y

Yalçın Çakmaklı G PP5-002

Yalçın İ

Yaraş $T$

Yardımcı AG

Yargıçoğlu Şahin B

Yarman Vural FT

Yavuz M

Yazihan N

Yemişçi Özkan M

Yener $\mathrm{G}$

Yener GG

Yerlikaya D

Yilar S

Yildirım E

Yildırım EA

Yildirım L

Yildiz M

Yilmaz A

Yılmaz Ö

Yırıkoğulları H

Yorulmaz $\mathrm{H}$

Yörüten M

Yurdakoş E

Yurdalan F

Yurduseven $\mathrm{K}$

Yücedağ $\mathrm{H}$

Yücel N

Yüksel O
PS6-3

SS2-C01

PS11-2

PS11-1

$11-3$, PP5-005

PS7-1, SS1-B04, PP7-007

SS3-A06

PS5-1, PS5-2, PS5-3, PS5-4, PP8-003

PP5-031

PS5-1

PS5-1

PP8-004

PP1-001

PP2-001

$\mathrm{SS} 2-\mathrm{C} 03$

SS2-A02

SS1-C02, SS2-B01, PP9-011

PS14-2

PP5-002

PS6-3, PS6-4, SS1-B05, PP5-023

SS3-B04, PP5-012, PP5-015

SS1-B05, SS3-B04, PP5-012, PP5-015

PP5-031

PS6-3, SS2-C01, SS3-A03

SS1-C02

PP1-005

PS12-3

PP5-024

PP7-004

SS2-C01

$\mathrm{SS} 2-\mathrm{B} 02$

PS15-2

SS1-C02

PP5-019

PS2-2

PP10-006

PP6-001, PP8-008

SS2-C05 